
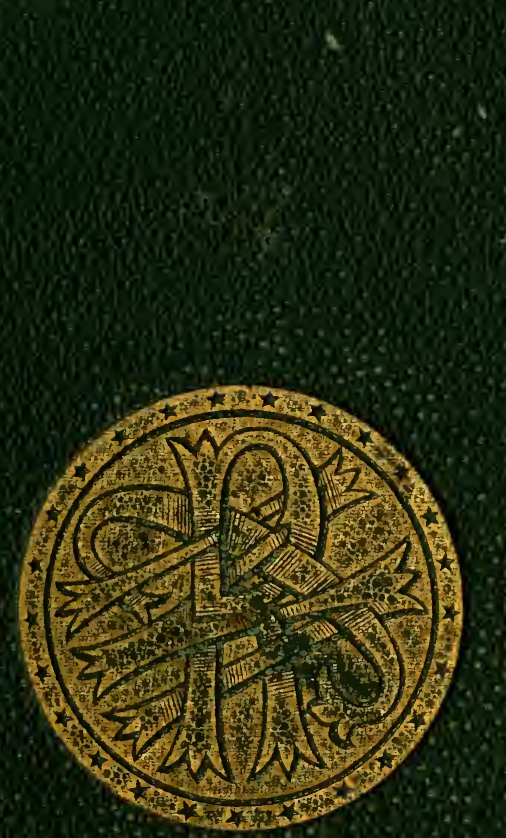

s

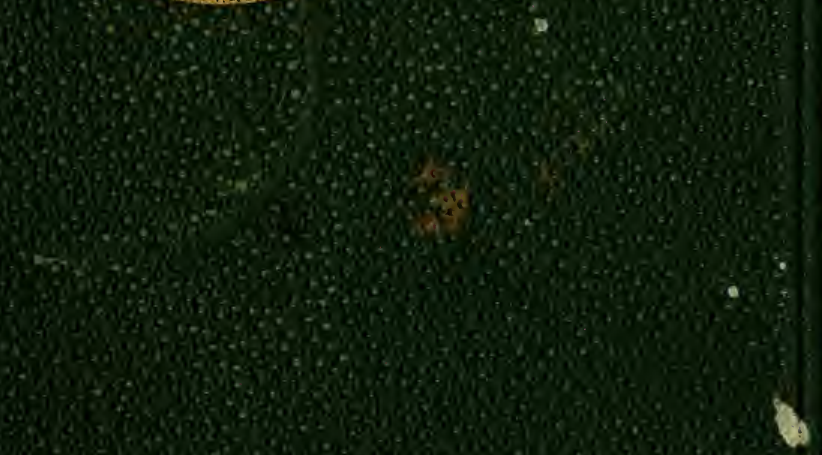




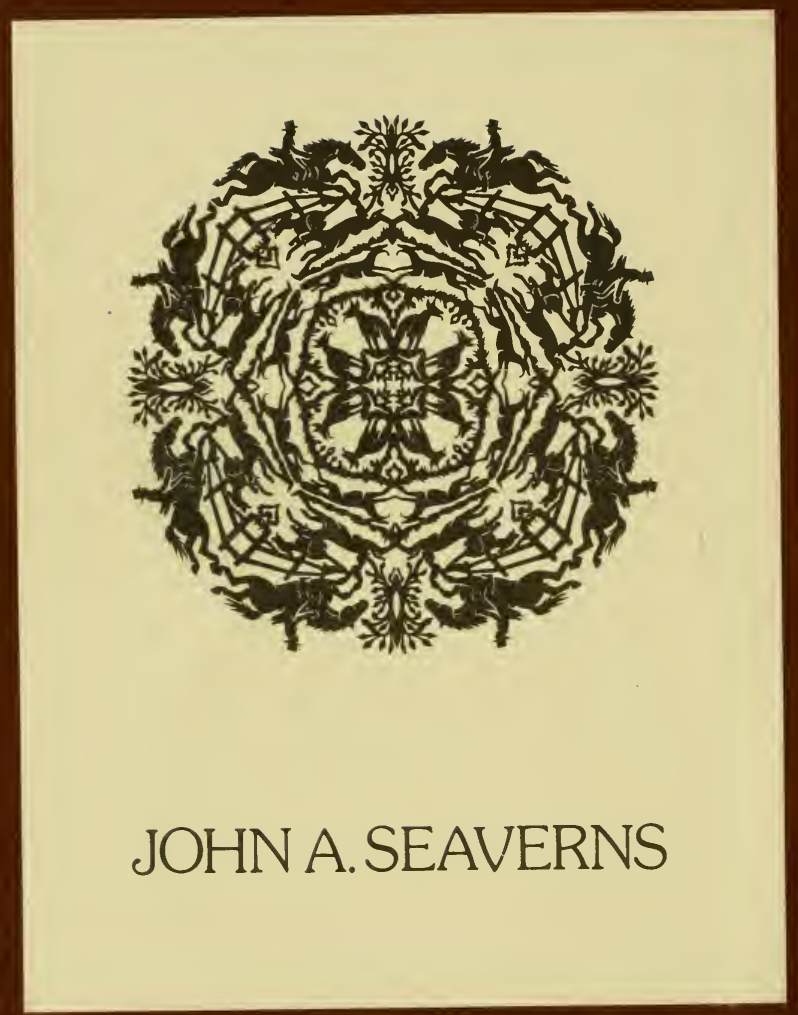


TUFTS UNIVERSITY LIBRARIES

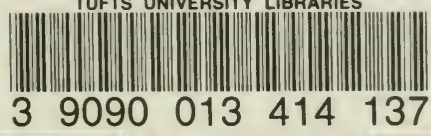


Webster Fas. Cummings: $x$ Tufts Unive
1. $\%$. . H. ... M Medicine

Vt. "raly Medicine at 
1
-1

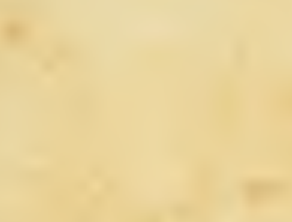

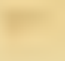

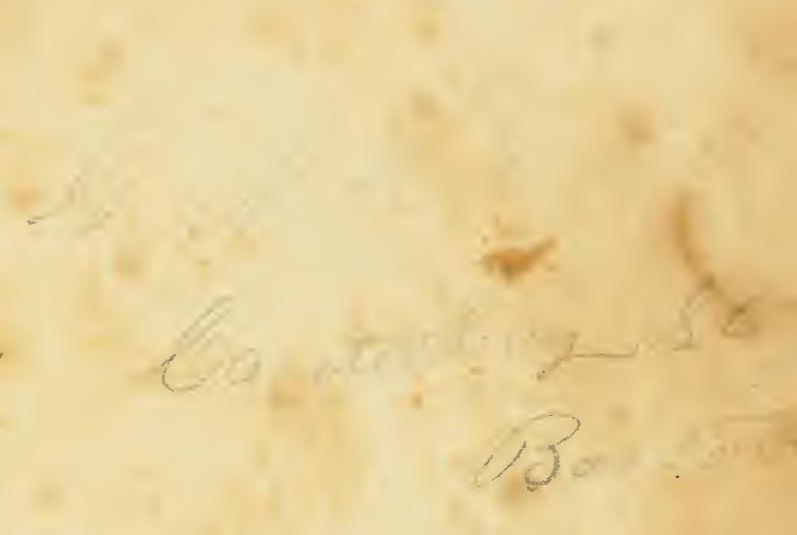

.

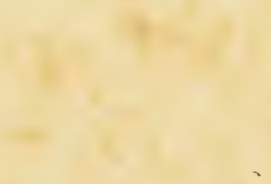

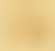

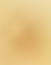

in

. 




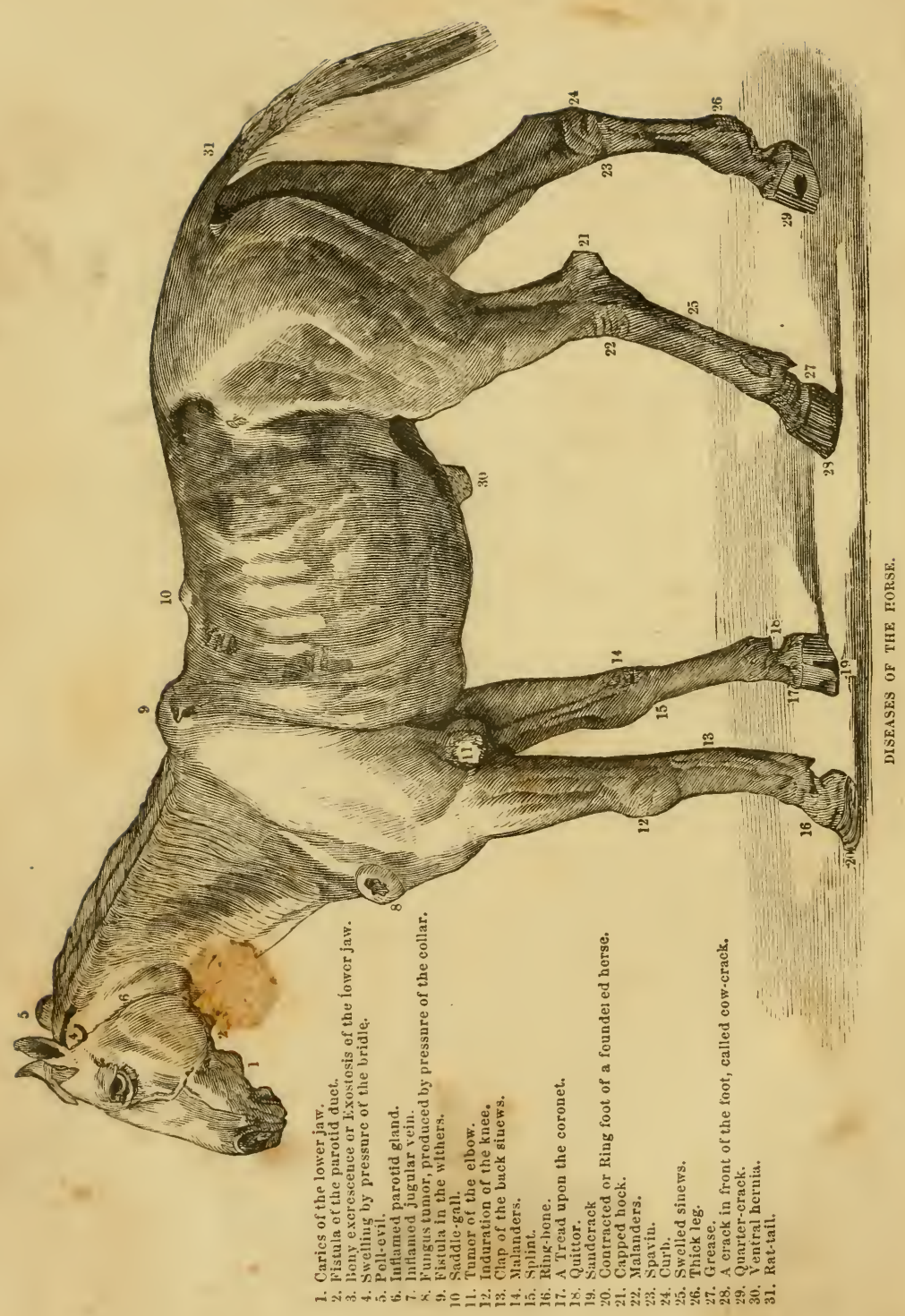




\section{I S E A S E S}

IN THE

\section{AMERICAN}

\section{Stable, Field and Farul-Yard,}

\section{CONTAININE}

A FAMILIAR DESCRIPTION OF DISEASES,

Cbeir dature, Canse, and Ẽmmptoms,

The Most Approved Methods of Treatment, AND THE

PROPERTIEX ANI UAF OF REIEDIEX, WITH DIRECTIONS FOR PREPARING THEM.

EXPRESSIY ADAPTED FOR

JHE AMATEur, FARMER, AND BrEeder.

BY ROBER'T MCCLURE, M.D., V.S., Editur of "Stonehenge, HcClure, and Harrey on the Horse,"
\&c., dc.

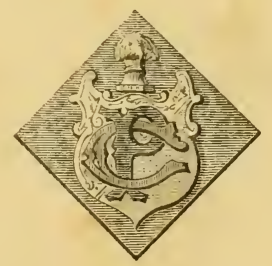

PH I L A D ELPH I A :

PORTER \& COATES,

No. 822 Chestnut Street. 
Entered according to Act of Congress, in the year 1866, By ROBERT MCCLURE,

In the Clerk's Office of the District Court of the United States for the Eastern District of Pennsylvania. 


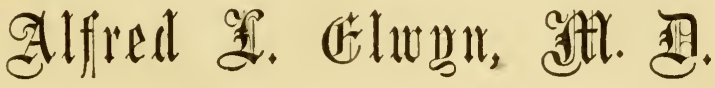

THIS WORK IS MOST RESPECTFULLY

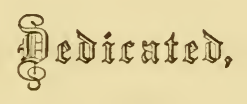

IX

GRATEFUL ACKNOWLEDGMENT OF GENEROUS SUPPORT

IN EFFORTS MADE TO ADTANCE TIIE CAUSE OF A SOUND SYSTEM OF ANIMAL MEDICATION,

BY THE AUTHOR. 



\section{PREFACE.}

Trins book is founded on a course of lectures delivered in the Veterinary College of this city, and is divided into three parts:-1. The diseases and treatment of the horse. 2. The medicines, prescriptions, and preparations used in veterinary practice. 3. The diseases and treatment of the ox, together with the treatment of diseases incident to milch cows and calves. To render the work as complete as possible, according to its title, an essay on diseases of sheep is added, thus forming a farmer's veterinary library and cyclopædia. The work is gotten up specially for the amateur, farmer, and stock breeder; at the same time, however, the veterinary surgeon can learn much from it which will be useful, although some of the doctrines may appear novel to him. The diseases and treatment of each animal are treated of under their most popular or trivial names, and in alphabetical order. The subject matter throughout the 


\section{vi PREFACE.}

book will be found truthful, concise, to the point, free from literary niceties or labored efforts; but frowns upon the destructive and injurious system of bleeding, blistering, physicking, firing, and low feeding or starving animals suffering from disease or accident. If this work, then, should enlighten the minds of men to a full understanding of the injurious teaching of old authors in new dresses, and point out a sound, safe, scientific, and successful system, the author will have been amply repaid for whatever time, trouble, and expense he may have incurred in presenting it to the public.

The author thanks Mr. Jas. B. Rodgers for the perspicuity of the letter press and manner in which he has executed the work intrusted to him.

Philadelphia, September, 1866. 


\section{INTRODUCTION.}

\section{Causes of Disease.}

Scientific men give three names when they speak of the causes of disease-exciting, predisposing, and proximate. The first may justly be termed the originators of disease; by the second is meant those more easily acted upon by causes that a more healthy animal would resist altogether; and the third is almost the disease itself. Of the causes with which we are acquainted, not many of them are alike, and their effects, that is, the disease, just as diverse.

These causes are named in the following table:-

1. Electric, and other conditions of the atmosphere.

2. Food and water.

3. Overwork.

4. Poisons-animal, vegetable, mineral, and zumins, or ferments.

5. Malformations, or badly-formed parts.

6. Age and decay.

7. Changes of temperature.

8. Hereditary influence.

9. Mechanical.

10. Starvation.

That I may be more clearly understood, examples will be given in the order above stated. 
The first is looked upon as the cause of the many diseases which take on an epizoötic form. The second, rusty straw, and musty hay and corn fed to animals with weak stomachs. Third, riding too far and too fast, overloading, etc. Fourth, animals drinking out of leaden troughs, where pieces of old iron may be lying in the bottom. Inoculation by the virus from a glandered horse, are illustrations of animal poisons, zumins, or ferments. (See Glanders.) Fifth. A horse with point of hock inclined forward, which is the originator of curb. Sixth. An old horse or cow, with no teeth to chew its feed. Seventh. Taking an animal from a warm and comfortable stable, and exposing it to a cold north-eastern storm. Eighth. A flat forehead, transmitted from parentage, thus preventing a full development of the brain where the optic nerve is given off from the brain, thus insuring blindness about the seventh or eighth year, and sometimes earlier. None need be told of the disposition of the course-bred Canadian horse to become affected with disease of the bones, mostly in the form of ring-bone, (which see.) Ninth. Stone in the bladder, and calculi in the bowels. Tenth. Besieged garrisons, fortresses, when crops have failed, and famine.

\section{How to Observe Diseases.}

We are sometimes asked how it is that we know so exactly what the disease is that this or that animal is affected with, as it cannot speak and narrate its ills and its aches. To this question we might repeat a common truism, "A shut mouth tells no lies;" therefore, nobody is deceived. Nature has but one set of weights and measures, and these only should be used. Thus, if a horse have a corn or 
bruised heel, he will be as sure to go lame as he would with an ordinary sprain. The difference is, that he not only stretches out the sore foot, but he elevates the heel from the ground, and will not set his foot flat to please, or it may be said to deceive any one. The uneasy eye, the anxious expression, and the sharp, peculiar look, tell its tale of suffering, and bear testimony to a description so faithful and true, that every man should understand how to interpret them.

\section{The Principles of Disease.}

Congestion.-By this term is meant an undue flow of blood into a part, and remaining in it. The blood-vessels lose the power of contracting and emptying themselves, as when in health. Congestion is not accompanied with inflammation, as some suppose, and may exist without irritation. Irritation is only present when the blood passes more rapidly into a part than its vessels can carry it out, and inflammation only is present when more fluid is thrown into the vessels than they can get rid of.

Irritation.-This peculiar condition is the result of increased sensitiveness, or an exalted action, accompanied with quickened beating of the heart and pulse. When we apply the term to special cases, it will be better understood, as irritation of the bowels producing diarrhœea, of the bladder frequent passing of urine, of the eye causing an increased flow of tears, and of the throat giving rise to cough.

Inflammation differs from irritation, inasmuch as it is more painful. There are three varieties-acute, sub-acute, and chronic. Inflammation has also three terminations:- 
(1.) Resolution; that is, it gives way, or is relieved before any alteration has taken place in the part so inflamed.

(2.) Suppuration, or the formation of pus.

(3.) Mortification, or death of the part affected, and the subsequent death of the animal. Inflammation is characterized by four conditions, or phenomena, as they are called-pain, heat, redness, and swelling. Inflammation assumes different degrees of intensity, modified by the cause, and the part or organ affected. When it is situated in the windpipe, (bronchitis, which see,) lungs, or bowels, great uneasiness and disturbance are manifest. In a few hours this condition gives way to depression.

Depression.-Many diseases of an inflammatory type, when their course is run, leave the system in a state of depression, or a low condition of vitality. How necessary, then, that this should be properly understood when treating inflammatory diseases, as one pint of blood taken from a large horse would, under these circumstances, cost him his life! Rather anticipate the weakness by supporting and husbanding the strength, so as to overcome disease.

There are two great divisions of diseased action, which it will be well for farmers, and others interested in the welfare of their animals to know, and these are:-First. The exalted, or as it is called sthenic, or commonly known by the term inflammation, and require for treatment, not bleeding, but medicine having the power of overcoming the exalted condition, by controlling the fast beating of the heart and pulse. This is easily done by the power of such agents as aconite or veratrum, (which see,) and without in any way impairing the strength and constitution of the animal. The second, or depressed condition of 
disease, and known as the asthenic, requiring a treatment opposite to the above variety, namely, iron, gentian, etc., (which see,) to add to the strength of the animal, and quality of the blood. An improved diet, and in greater quantity, will, in many cases of depression, not only cure the disease, but remove the cause also.

\section{FEVER.}

There are four stages observed in fever:-

(1.) Weakness, loss of appetite, and low spirits.

(2.) A shiver, or chill, uneasiness, flanks move quick and short, nostrils more or less distended, one leg or ear hot, and the other cold.

(3.) After a time the coldness is succeeded by great heat and thirst, costiveness, urine scanty and high-colored, mouth hot and dry.

(4.) When the fever has lasted for a longer or shorter time, the skin becomes more moist, the bowels and kidneys act more freely; the pulse becomes more full, although not less frequent, and the mouth more moist.

When fever is accompanied with disease of the lungs, liver, or other organ of the body, or after an accident of any severity, it is then called symptomatic fever.

Fever is called idiopathic, when not accompanied by disease or accident. From the days of Galen to the present time, many theories have been advanced to explain its proximate cause, but none seems to satisfy the philosophical student, and it still belongs to the unsolved problems in physiology.

Diseased Secretion.-A good example of what is here meant may be found in the discharges from the nose of animals affected with cold, influenza, and glanders. The 
salivary gland may secrete too much fluid, as in the case of horses eating second crop clover. We call this salivation.

Increased Secretion.-In health, serum is only supplied in sufficient quantity to keep the surfaces moist, the absorbent vessels preventing accumulations. In the cavities of joints this secretion is often too large, causing enlargements, of which a good example is seen in swellings of the hock-joint, called bog spavin, and in thorough-pin.

\section{The Pulse.}

The pulse of the horse and the ox is felt on the inner angle of the lower jaw, as being the most convenient place. The state of the pulse tells the condition of the heart, whether the disease is of an exalted or depressed character, or whether sickness is at all present. The pulse is more frequent in young than in old animals. In the fullgrown and healthy horse it beats from thirty-two to thirtyeight in the minute; in the ox or cow, thirty-five to fortytwo; in the sheep, seventy to seventy-five; and in the dog, from ninety to ninety-eight. In inflammations and fevers the frequency of the pulse is increased. In debility and depression it is slower, but sometimes quicker than natural. There are the quick pulse, the strong, the sharp, the regular, the intermittent, and many other varieties, both fanciful and real, which few persons can appreciate. The pulse of inflammation and fever numbers from seventy-five to eighty beats in the minute; and in great debility, as in the last stage of glanders, accompanied with tubercles of the lungs, the pulse will number one hundred beats per minute. 


\section{Breathing.}

A good-sized, healthy horse, will take one inspiration to three of the pulse beats. When the breathing is more frequent or slower, and when irregular, or difficult and laborious, there is then disease; although we sometimes see the breathing quickened and short, when no disease is present. Both the pulse and the breathing will be quickened by exposure to heat, as in a stable up stairs, and exposed to an August sun. By removing the animal to a stable not so situated, the breathing and the pulse will be greatly lessened. Hence the advantage of placing animals in a cool and airy place when they are unwell. It saves a great waste of their strength and vitality, thereby enabling them to throw off the effcets of disease.

\section{Treatment of Disease.}

The antiphlogistic plan of treating disease was derived from a theory now entirely exploded, and almost forgotten. Repeated bleedings, blistering, physicking, and starving on low diet, are some of the measures entering into the general plan which has destroyed more life and property than all the wars, ancient or modern.

Bleeding, in domestic practice, is almost discarded, and in veterinary practice it should never have been employed. And if this fact shall be the means of opening the eyes of those interested (and who is not?) in the health of the animals supplying us with meat, and the horse, (a willing and a faithful help,) to the injury done by bleeding in health or disease, I shall be well rewarded. Avoid these measures, and substitute a rational and successful system of treating the diseases of your animals. Ascertain whe- 
ther your horse is suffering from a disease of an exalted or inflammatory kind; substitute aconite, pure air, and cold water for bleeding, and in a few hours you will have no cause to regret the change. If the disease be of a depressed kind, accompanied with weakness and debility, give nux vomica, iron, and a generous diet. If the disease be an eruptive fever, give sulphite of soda to purify the blood. In rheumatism, administer colchicum and carbonate of soda. In mange, apply the sulphuret of potassa to the skin, to destroy the small insects which cause the trouble.

In hard swellings use the preparations of iodine, to cause their absorption. In lameness, allow absolute and entire rest, and apply hot or cold applications and slight irritants to the parts, to remove the products of the sprain. Ascertain the cause of disease, and having found it, have it removed, and the effects will cease. If the animal be costive from eating dry, concentrated feed, remove it, and give green feed or bran, but do not give physic. If diarrhœa be present, leave it, at least for a time, to itself, as it is nature's plan of getting rid of the offending matter. But, if it should continue, chalk and opium, as an astringent, are what is wanted. The reader cannot fail to see how simple, and his experience will demonstrate how successful these measures are in arresting and curing the diseases of all our domestic animals.

\section{Vis Medicatrix Naturæ, or how Diseases are Cured without Medicine.}

Intelligent persons have no difficulty in recognizing in the constitutions of animals and men a power of selfrestoration, which is capable of resisting the influence of 
disease. It is this power that heals wounds, unites broken bones, and supplies lost substances. Diseases are not unfrequently efforts in this direction, and to stay the action of hurtful material when admitted into the system, or the eye, for instance, receives a particle of sand or hay-seed, the weeping of the secretions of pus are remedial measures to rid the eye of the offending body. Poisons are good examples of the manner in which animals will cure themselves. When poison is taken into the stomach, irritation of the bowels is set up, followed by purging, as an effort to get rid of the poison. Nature, however, is not always successful, and the animal may die from the violent action set up. Again, a sprain will be cured by this very power, provided absolute and "entire rest be allowed to the sprained part, without any interference from medicine or art. The remedial powers of nature often require assistance, as, for instance, in cases of debility, when the blood is becoming too watery. A few doses of iron, and in many cases a little extra food, will enable the sanative powers of the constitution to effect a complete cure. Often the removal of an animal from the sphere of exciting causes of disease, will cause the effect to cease, and the power of nature will cure the affection. Hence, many persons reflect upon the many instances when apparently severe cases of sickness were cured by some simple substance, and much credit given to a power it never possessed. Where the powers of nature are left to perform a cure, let the strength of the animal be maintained, because if that fail, where is the chance of recovery? Blood-letting and physicking are powerful and depressing agents, so much so, that when carried to any extent, few, if any animals, by the little power that may be left, will cure themselves. It is this knowledge that 


\section{6}

INTRODUCTION.

enables Homœopathists to continue their practice; for if it were not for this power in the constitution in each and every animal, Homoeopahists would have long since ceased to practise their peculiar art. If farmers and owners of horses and cattle will only cease to bleed, and pour nostrums down the throat of their stock, and learn to rely more upon the great curative that God has implanted in the constitution of all His creatures, as a power in protecting their lives when attacked by disease, it will surely be infinitely more profitable and pleasant to them. In curing disease, medicine and art should be directed to assist the powers of nature to overcome disease,-nothing more. 


\section{DISEASES OF THE HORSE.}

\section{PART FIRST.}

\section{Their Nature, Symptoms, Cause, and Treatment.}

An alphabetical classification of disease is the only arrangement adapted to popular instruction and domestic use. As some diseases have more than one common name, a few references are all that may be necessary to find the particular disease wanted. In every disease the treatment I have first recommended should be tried; and, if it be not successful, the next in order will be taken. Also begin with the smallest dose, increasing, diminishing, or withdrawing it altogether, as the case seems to require. I do not think it necessary to quote authorities to substantiate what is said in regard to this or that medicine as a remedy, as the plans and remedies are those employed by the profession.

Abrasion signifies to tear off, and is applied to the skin when it has been rubbed or torn off, and to the lining membranes of the nose. The treatment will be found under that of bleeding wounds.

Abscess.-This is also called, by some persons, a 
beeling-a formation of matter or pus under the skin, as the result of inflammation, either acute or chronic. Sometimes abscess in bone is seen, also of the liver and the brain; and, indeed, no part or tissue of an animal is exempt from it.

Symptoms.-Pain, heat and swelling; a projection or prominence on the swelling from which the hair falls off, disclosing a yellow, white and soft part upon its apex. In a common abscess of this kind, it will only be necessary to hasten the formation of the pus by applying poultices of flaxseed or some other soft substance to the part, and when the point is soft and evidently contains ABSCESS fluid, make an opening on its lowest dependent point with a sharp knife, so that the discharge will flow out of itself, and then apply

$$
\begin{aligned}
& \text { Rain water . . . . . } 1 \text { ounce. } \\
& \text { Chloride of Zinc . . . . } 6 \text { grains. } \\
& \text { Mix and apply to the wound twice a day. }
\end{aligned}
$$

It is not advisable to open an abscess too soon, or before the pus has properly formed. (See Articles Strangles and Fistula.)

Acari.-(See Mange.)

Accidents.-(See Sprains, Bruises, Bleeding, and Wounds.)

\section{Aconite.-(See Medicines.)}

Amaurosis.-Glass eye. (See Eye Diseases.)

Anæmia.-Deficient or bad blood.

$$
\text { Anchylosis.-(See Spavin and Open Joints.) }
$$

Aneurism.-A pulsating tumor, produced by the rupture of the inner coats of the vessel, and the blood getting 
between it and the outer coat. They manifest themselves in many parts of the body. An expert surgeon is only capable of remedying it, as great danger of bleeding to death would result from opening a tumor of this kind.

\section{Apoplexy.}

Symptoms.-The animal falling suddenly, loss of feeling and the power of motion, and breathing deep and slow. In most cases the horse gets up again, shakes himself, and proceeds on his journey almost as if nothing had happened, but it will shortly be seen that the animal is not so lively as formerly, and that it will afterwards be unsafe to use him, especially for a family carriage, as in a fit of this kind the horse may become entirely unmanageable, and can only be controlled by a power stronger than his own. Horses subject to disease of this kind, will, at times, be observed to look sleepy, with a slight knuckling of the hind pastern joints, accompanied with stiffness of the hind quarters and loping of the ears. All these symptoms are seen in brain disease, as Staggers, Megrims, and Epilepsy. (Which see.)

Causes.-Breaking of a blood-vessel, effusion or water on the brain, producing pressure, too small a collar on a thick-necked horse, interfering with a free circulation of the blood to and from the head, effects of the sun-sunstroke. (Coup de soleil.)

In severe cases of Apoplexy, many never have the use of their legs again, by their remaining palsied. (See Palsy.)

Treatment.-Apply chopped ice to the head in bags, and secured by proper fixings. Keep up a free circulation in the legs by rubbing and woollen bandages, or warm water cloths, and renewed every half hour. Then apply a small blister of 


$$
\begin{aligned}
& \text { Spanish Fly . . . . . } \frac{1}{2} \text { drachm. } \\
& \text { Hog's Lard . . . . . . } 2 \text { drachms. }
\end{aligned}
$$

Mix them well together, and rub the salve well in by the hand on the part just behind the ears.

Do not bleed, as that measure will only insure effusion, and, as in the brain, ultimately cause death or dumbness. (Which see.)

If there be much exaltation or excitement, give fifteen drops of the tincture of aconite root every four hours, till five or six doses are taken. If thecontrary condition be present, that is, depression, give from ten to fifteen drops of the tincture of nux vomica four times a day, for a few days, or a week, if necessary. These medicines are best given in cold water, when the animal will drink it; if not, mix with a cup of water, and drench out of a stout-necked bottle. Feed the horse generously and well.

\section{Aphtha.-(See Mouth Diseases.)}

Atrophy.-Wasting and shrinking of a part of the muscle, as is seen in sweenie of the shoulder; consumption and disease of the mesentery, and also palsy or paralysis of the hind legs, from which the muscles of the hip will be seen to have fallen away.

Causes.-The parts deprived of their proper use, action or function. A long-continued corn on the foot of a horse, depriving him of the proper use of that limb, will cause sirinking of the sub scapularis muscle of the shoulder.

Treatment.-Removal of the cause, and restoring the functions of the parts to their proper condition. In case of many joint diseases, it will be necessary to have the horse walked before he is cured, to prevent too much wasting of the parts from long standing and want of use. 
Back sinuses.-(See Sprain.)

Baldness.-(See Skin diseases.)

Belly Ache.-(See Colic.)

Big Head.-(See Head diseases.)

Bishoping-Is the name of an operation performed upon the front or nipper teeth of horses that are more than eight years old, for the purpose of imitating the mark of the teeth of young horses, in order to deceive those persons who are supposed to be poor judges in the age of horses. It can rarely deceive any person of ordinary intelligence. The general appearance of the horse will soon tell, if he be an old or young animal.

Bite of Mad Dog.-When any reasonable suspicion exists that the dog is mad, have the parts completely washed out with cold water, if possible forced with a syringe or hydrant, so as the water will find its way to the bottom of the wound, and wash out any virus that may have lodged there.

If the bite be upon the leg of man, horse or other animal, or man's arm or finger, a Tourniquet or soft rope or cord should be tied tightly around the leg above the bite, till other measures are used to have the bite purified. After the cord is properly applied, have the parts washed out; next either cut a portion of the flesh from the top, sides and bottom of the wound, or apply caustic to the parts. The nitrate of silver is possibly the best for this purpose. A few drops of nitric, hydrochloric or sulphuric acid may be dropped into the wound, and by the burning properties of these articles the destructive character of the poison will be destroyed.

After these measures have been satisfactorily per- 
formed, the wounds should be treated as for common wounds with simple ointment (see prescriptions and medicines.) The bite of other rabid or poisonous animals should be treated in the same manner. Persons have been bold enough to have allowed themselves to be bitten by mad dogs no less than seven times, then applying the nitrate of silver as a caustic to the bites, and yet never became affected with the canine disease. However, too much dependence should not be placed in any one measure as a protection in such cases. The great protection is, do not allow yourself to be bitten at all, if it can be avoided.

Bites from a healthy dog will never produce madness, even although the animal go mad in a year or two afterward, so keep your mind easy on that point.

\section{Bladder Diseases.}

1. Inflammation.-This condition of disease is sometimes met with, but is rare in horses.

Causes.-Irritating substances or foreign bodies in the bladder.

Symptoms.-Constant desire to make water, pain, straddling or walking wide with the hind legs, great tenderness under the belly.

Treatment.-Inject a little warm oil into the bladder. This can only be done by an expert and with a proper instrument. Give 25 drops of the tincture of aconite root every 4 hours, till 6 doses are given, to keep down pain. Allow flaxseed tea to drink, or drench the horse with it, which has an excellent soothing effect. Give plenty of cold water to drink.

2. CALCUlI.-This variety of stone is sometimes found in the bladder and kidneys of horses. This may be said to be the gravel of the horse, although not so common 
as in man, but is equally troublesome, and requires for its cure a formidable operation called Lithotomy, an operation of no great magnitude to an expert surgeon, but can scarcely be undertaken by an unprofessional person, even though a description of it were given.

\section{Bleeding.}

1. BleEding.-An operation for the drawing of blood from the body, either locally or generally. As before stated, it is almost entirely discarded from domestic practice, and should never be used in the treatment of diseases of animals, however much the adherent of an exploded and an erroneous system may doubt it. Medi-" cines will be described in this book that will not only insure greater success in saving a very much greater percentage of sick animals, and with less trouble in a much shorter time, and without in any way impairing the sanative powers of the animal's constitution.

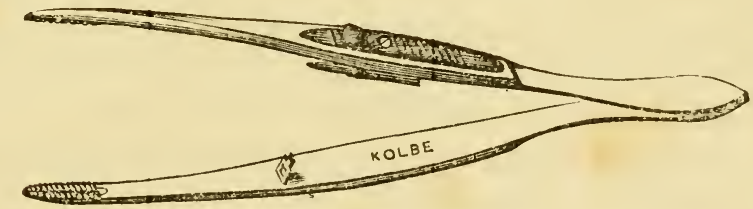

FORCEPS FOR LIFTING BLEEDIYG AND WOUNDED BLOOD VESSELS SO THAT THEX MAY BE TIED.

2. BleEding From Wounds.-If the wound be a simple one, and not on the inside of a leg where the large bluod vessels are situated, all that will be necessary to stop it will be a small piece of cotton or soft cloth placed in and over the wound, and secure it for a few hours by a broad bandage, not too tightly applied over it, or, if preferred, touch the mouth of the bleeding vessel with a piece of iron previously immersed in boiling water or in 
the fire itself. The surgeon's plan would be to get hold of the mouth of the vein or artery with a pair of artery forceps or small tongs, to hold it so that he can tie a piece of saddler's silk around it. If the wound be on the inside of the hind or fore leg, and the blood of a scarlet color (see Blood,) place pads of cloth and bandage pretty tightly over it, and run for a good surgeon, and tell him what the trouble is, so that he will go properly prepared for his work.

3. Bleeding from the Air Passages and Lungs.Observe the color of the blood discharged from the nose or mouth, as the veins of the lungs convey blood similar to the arterial blood of other parts of the body.

Causes.-The laying bare, and the rupture of small vessels, and the structure of the lungs, breaking down as in consumption, and some cases of glanders and coryza.

Treatment.-Support the strength by the mineral acids, (see acids) and small doses of aconite to lessen arterial circulation. Bear in mind in cases where the structure of the lungs is falling to pieces, no power or art can arrest it. Hence, the incurability of consumption.

4. Bleeding from the Skin.-This disease is sometimes called purpura.

Symptoms.-After general uneasiness, some pain, fever, and swelling of the legs and other parts of the body; tumors varying from size of a cranberry to that of a pigeon's egg, often running together, forming large patches from which blood is oozed out in great quantities, giving rise to much aebility. The contagious typhus or rinderpest of cattle, bears many resemblances to this disease of the horse.

Treatment.-Feed the animal on the best food that can be procured, and pour forty drops of commercial 
sulphuric acid in half a bucket of cold water three to four times in the day. Then get two ounces each of the sulphate of copper and gentian root in powder, and divide into eight powders, and give one night and morning in the feed.

Apply to the bleeding surfaces and sores, a liniment composed as follows:-Olive oil three ounces, creosote one ounce; mix and use once in the twenty-four hours.

\section{Bloody Urine.-(See Kidney Diseases.)}

Blood.-Bloor is observed to be of two colors, namely, red, or almost of a bright scarlet. When blood of this color is issuing from wounds in jets or jerks, it is considered more dangerous than if it were of a dark red, or venous blood. The first is direct from the heart itself, and the other is from a more remote and less dangerous part.

It may be interesting to know, that red globules are more plentiful in blooded or well-bred horses than in horses of a coarser kind, which accounts for a curious fact observed in the difference of vitality. Thus, a blooded horse bears up under diseased action, and is cured, whilst a western or common horse will die under the same disease.

The fluid portion of blood is called liquor sanguinis, in which the red globules or spheres float. When blood is drawn from the body, it divides into two parts: the solid is called clot, and the other is the serum. This serum was once relied upon, and is still by the ignorant, as showing the existence of inflammation. It is by the blood that the strength, wear, and tear of the system is kept up. The heart is the organ by which the blood is forced through the body. If the blood be thin and wa- 
tery, it is called hydronemia, (see Dropsy.) If pus be in the blood, it is then called pyemia. (See Glanders.)

Boils.-(See Saddle or Harness Galls.)

Bots.-(See Worms.)

Bowels, Disease of.-(See Costiveness, Diarrhœa, and Dysentery.)

1. Inflammation of the Bowels.

Symptoms.-Acute pain in the belly, and continuous, getting no intervals of rest from the pain. Rolling, pawing, and shifting about, sweating, and breathing fast, with great fever, exaltation, and excitement. A fearful disease. Happily not so frequent as formerly.

Can only be mistaken for colic (which see.) In colic there are times of ease from pain, but never in this disease.

Causes.-Exposure to cold, drinking cold water in great quantities when hot, calculi, or hair balls in the bowels, costiveness, diarrhœa, and as a sequel to colic, lead and other poisons, (which see.)

Treatment.-The first thing to be done is to lessen or destroy pain. Give a large dose of the tincture of aconite root, say thirty drops, to be repeated in two hours. Apply blankets rung out of boiling water to the belly, and renew them in about twenty minutes.

Give injections of warm not hot water, soap, and a handful of table salt every half hour. Continue the treatment while there is enough strength remaining.

Bleeding will only insure and hasten death, and purgatives are too slow to act-the horse is either dead, or will be before any response can be had from them.

Brain Diseases. The brain, and its coverings, or 
membranes, are subject to inflammations of every degree. (See Apoplexy and Staggers.)

Breaking Down.-This accident means or consists in rupture of the tendons and ligaments, and occurs at once when the horse is at full speed.

Symptoms.-The horse stops suddenly, or perhaps stumbles and falls; gets up, but stands on his fetlocks, the toe of the foot turned up, and the sole of the foot, as it were, looking at you.

Treatment.-If the fetlock comes entirely to the ground, not much can be done; and when it does not, contraction of the leg takes place, and requires division of the tendon. (See Tendeotomy.)

Breathing Short.-This is a symptom of irritation, inflammation, debility, weakness, oppressions of every kind, and fever, (which see.)

Breeze Flies.-This is the fly supposed but erroneously to be the one that deposits the ova or eggs, which generate bots in horses. (See Worms.)

\section{Brittle Feet.-(See Foot Diseases.)}

Bronchi.-This term means the windpipe, and communicates and carries the atmosphere to and from the lungs. And is the seat of disease, and is affected more or less in all cases of colds and inflammations, whether of the lungs or their membranes-the pleura.

\section{Broǹchitis.}

Infammation of the Air Passages or the Parts endmerated Above.-This is a very common disease among horses, and is confounded by most horse doctors with inflammation of the lungs, distempers, and colds; whereas, it is distinguished from inflammation of the 
lungs by its seat, and from the others by as great a dissimilarity.

Bronchitis occurs in various degrees of intensity, and should at least be described under two heads, notwithstanding the one distinction should run as it does sometimes the one into the other.

1. Acute Bronchitis.

Symptoms.-Ushered in by a chill, fever, harsh or painful cough, loss of appetite, heaving at the flanks, mouth hot and dry. In a day or so, a discharge of pus or inatter will be observed from one or both nostrils.

If bleeding, or other severe measures be used, the horse will assuredly die, not so much from the disease, but from maltreatment. Better, in a disease of this kind, let nature have her way, and give the horse a chance for his life; for in the other case, he has absolutely none whatever.

Treatment.-First, the horse should be treated as for fever. Place the horse in an open or airy place, embraced by the word "comfortable." Obviously it would not be comfortable to place a sick horse in an open place, with the thermometer below zero. In July or August, it will not be necessary to burden the animal with blankets.

Give him from fifteen to twenty drops of the tincture of aconite root every four hours, till six doses are taken. This will relieve the fever, breathing, and the hot and dry mouth. Give plenty of cold water to drink, as pain and fever require plenty of fluid, as all the secretions are dried up. Let the animal have a little grass, if it can be got: this will relax the bowels, and cool the stomach. On the second day, the following medicines may be given, that is, if they be necessary:-Powdered gentian, 
root two ounces, powdered nux vomica, one ounce. Mix, and divide into six powders, and give one powder morning, noon, and night. These medicines will prevent debility and depression, and the pouring out of fluid or serum into the legs, sheath, belly, and breast. After the fever has been removed, allow good feed, and a fair quantity of it. Such treatment will not only cure the horse in five or six days, but the horse is nothing the worse, only the loss of a few days' work.

2. Chronic Bronchitis.

Symptoms.-A confirmed cough, more or less severe, and a discharge from the nose. The cough worse in the morning, and after drinking water.

Causes.-Maltreatment of acute cases of bronchitis, and where the attack has been prolonged beyond a reasonable time, causing ulceration or thickening of the windpipe.

Treatment.-Give extract of belladonna, half drachm, powdered digitalis, half drachm, three times in the day, morning, noon, and night, for a few days. If no good seems to have been done, a different plan will have to be adopted, namely, feed the animal well, and give sulphate of iron, two drachms in powder, gentian root, two drachms in powder, twice in the day for two weeks. By this time the absorbent system will be pretty powerful. Then apply the following salve or ointment, well rubbed in down the course of the windpipe, once in the week. Lard, one ounce; red iodide of mercury, one drachm. Mix. This will cause whatever thickening may exist, to be absorbed or taken up. Lard or oil will have to be applied once per clay on the place where the ointment was applied, to prevent the skin from cracking. If the animal be debilitated, give occasionally sixty 
drops of commercial sulphuric acid in half a bucket of cold water to drink.

Bronchocele. - Pronounced "bronch-seal,", is an enlargement of the thyroid gland, and is situated on and in front of the windpipe, about three inches from the lower jaw. In horses it is only an eye-sore or blemish.

Causes.-In the horse it is unknown, nor is it determined what office or use the thyroid gland performs in man or animals.

Treatment.-Apply with friction by the hand once a week, biniodide of mercury, one drachm; hog's lard, one ounce. Mix, and make an ointment. Rub in a piece about the size of a hickory nut over and around the enlargement.

Broken Knees.-When a horse stumbles and falls upon his knees, and takes the hair and some of the skin off, this is called broken knees.

Treatment.-Wash and cleanse the parts from sand and dirt, and if the skin is ragged and torn, clip off with a good pair of scissors. After this is done, ascertain if there is any discharge from the sore of an oily substance, and if so get a smooth piece of iron, immerse it for ten minutes in boiling water, and apply it to the edges of the wound, so as to cause the parts to swell, and prevent the escape of the joint oil; for if this be allowed to escape, the ends of the bones will come against one another. Irritation and inflammation will be set up, and either destroy the life of the horse, or make an anchylosed or stiff joint. This is an important point to be observed, and that very early in all cases of broken knees 
or open joints wherever situated. After the hot iron has been applied, use the following wash twice in the day:-Sulphate of zinc, half an ounce, rain, or soft water, eight ounces. Mix. This will heal the sore, and prevent proud flesh from growing. This wash will answer for the more simple wound of the knee, and where there is no open joint. Do not apply bandages, as they will cause the whole leg to inflame and swell. Sometimes a kind of pouch will be formed by the lower edge of the wound, holding whatever pus or other fluid may escape. This pouch should be opened at its lowest bottom, so as to let the fluids out, and to prevent a bulge or permanent swelling remaining after the knee has otherwise healed. By attention to this, very little or no blemish or scar will be left to tell the tale of a stumbling horse, or a bad horseman.

\section{Bruises of the Sole.-(See Foot Diseases.)}

Burns and Scalds.-Injuries inflicted upon some parts of the body by the application of solid heat, is called burns; by fluid heat, scalds.

Treatment.-In horses the treatment is chiefly local; for in cases of fire in large cities, the generation of steam is sometimes so great, that it is inhaled by animals in the burning building: this is termed scalding. Not much can be done, nor is there much hope of a cure in cases of this kind. The utmost that can be done is to give plenty of cold water to drink, and keep down pain by means of aconite, (which see.)

For burns the best application will be, one pint of linseed oil, and half a pint of lime water, stirred together, or rather whipped (as the cook does with eggs,) till the mixture is like thick cream. This is to be applied to 
the burned places, spread on cotton or linen rags for a few days; then dress the sores with simple ointment. (See Medicines.)

Bursa Mucosa Enlarged.-(See Spavin and Wind Galls.)

Calculi.-Stone in the bladder.

Cancers. - This is a hard tumor, malignant in its character, at first small in size, but rapidly increases, and becomes ulcerated. The horse is not affected with so many varieties of cancerous growth as the ox and the dog, and even man himself. Melanotic cancer is most commonly seen in gray horses, or those that turn white with age. A small hard tumor is usually seen under the root of the tail, and about the anus.

When tumors of this kind are seen on gray horses, it may reasonably be expected that growths of the same character will be found inside, on the spleen, stomach, and liver. Horses so affected may work well for years, and may not for a month. The tumors increase in size, when cut into, they discharge a fluid as black as the ink of the cuttle-fish.

Causes.-As this disease is not seen in young gray horses until after they have almost turned white, it may be inferred that the cause results from the loss, or want of the usual pigment, or coloring matter, which gives color to the hair, thus depriving the animal of some protection from the air or sun. I think, however, that the true cause will be found to be, not in the loss of the coloring matter, but of its transfer from the skin and hair to the blood. Hence the inky color of the contents of the tumors. This opinion, which is my own, is still farther corroborated by the fact that if the pus be taken from an 
abscess, and injected into the blood of a healthy animal, carbuncles containing pus will soon manifest themselves.

Treatment.-Incurable; but when one of these tumors breaks, treat it as an ordinary abscess, (which see.)

Canine Rabies.-(See Hydrophobia.)

Capped Elbow.-This is an enlargement on the point of the elbow, just behind the shoulder, and on the side of the chest: sometimes it is in the form of a simple abscess, (which see.) But the usual form is that of an encysted tumor, or a fluid contained within a cyst of fleshy walls, which do not suppurate.

Cause.-The horse, when lying, rests the point of the elbow upon the heels of his front shoe. It is a symptom of disease of the leg, preventing the animal from properly flexing or bending the leg proper upon its thigh or arm. Hence, the elbow rests upon the foot.

Prevention.-Remove whatever disease may be in the leg, and place a pad of leather, or of coarse, heavy cloth, over the back part of the foot. Pads are made by Lacey \& Phillips, Harness makers, Phila.

Treatment. - Make an incision with a sharp knife through the skin, over the centre of the swelling, and carefully dissect the cyst from its attachments. This can be done with the fingers and a blunt piece of wood, flattened at its point like a butter knife, and no sharper. If the operator be timid, and think he cannot cut the cyst out, open it, and let out the matter, and inject, once per day, a little tincture of iodine, to kill the walls of the cyst, so that it will not fill again. (See Medicines.) A knife is only wanted to cut the skin. After the tumor is taken out, treat the part as a simple wound, by keeping it clean, and applying a solution of zinc, or blue stone. 
Capped Hock.-This is a soft swelling on the point of the hock-joint.

Causes.-Kicking in the stable or in harness, lying upon stone-paved stalls, and from being kicked by another horse.

Treatment.-Apply cold water cloths to the part for a few days, taking them off at night. After the heat and tenderness have subsided, apply, with rubbing, once every fifth day, for three times, if it be necessary, an ointment, composed of one drachm of iodide of mercury; and hog's lard, one ounce. Mix.

Carditis.-This is applied to disease of the heart. Heart disease is also known as the Thumps. Incurable. (See Heart Disease.)

Caries.-This term means an ulceration of the bone. The most frequent form of caries is seen on the lower jawbone; the teeth are next affected. If the upper teeth be ulcerated to any extent, a fetid (stinking) discharge will run from the nostril upon the side on which the diseased teeth are situated, which has been repeatedly mistaken for glanders by "horse doctors." If caries of the bones of the head exist, the swelling of the head will be enormous-Big-Head, or Osteoponosis-and not Osteosarcoma, as it is called by Mr. Jennings.

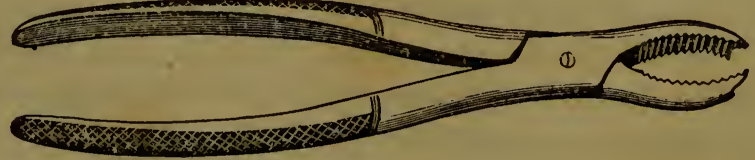

BONE FORCEPS.

Cause.-A disposition in the system to appropriate to the bones more calcareous or earthy matter than is required. 
Treatment.-If caries be confined to the teeth; have them taken out. When confined to the bones of the head, it is incurable. The animal will die of hunger, as he is unable to use his tongue and jaws, or gather and chew his feed. Happily, not a common disease in the Middle States. I have heard of many cases in the Western States. Arsenic and the hot iron are there recommended. Better let the horse die than increase his suffering, as he will die any way.

Castration.-This is an operation for the purpose of depriving the horse-colt of his entirety by the removal of the testes. It is a simple and safe operation. Any person having once seen it done, can do it also, if he have the resolution to do so. It has been recently demonstrated that castration can be performed on aged horses with as much safety as on those in colthood. This is attributed to the mode or manner of operation, namely, by an instrument called the Ecraseur. (See Instruments.)
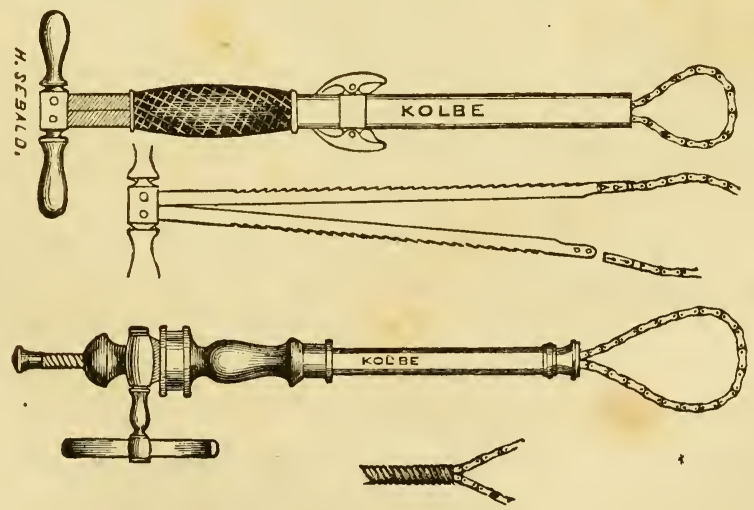

ECRASEUR, (OR NHW INSTRUMENT FOR CABTRATING COLTB.)

No clamps, no firing nor twitching, nor any trouble af- 
terward. The instrument is manufactured especially for this purpose, by surgical instrument makers in Philadelphia.

\section{Cataract.-(See Eye Diseases.) \\ Catarrh.-(See Coud.)}

Cautery.-This term is applied to the operation of searing a part with a red-hot iron. Happily, this cruel, and in many instances unnecessary operation, is becoming among the things that were. It used to be applied to sprains, ring-bones, and spavins. Since the discovery of the preparations of iodine, and their absorbent properties, the iron is little used.

Chest Diseases.-The diseases of the chest are many and important. In it are the heart, lungs, and great blood-vessels. The diseases of these organs will be found under Inflammation of the Lungs, or Lung Fever, Pleurisy, Coughs, Bronchitis, and Glanders.

\section{Chest Founder.-(See Founder.)}

Chill.-This term means a shiver, as if the horse were cold. This is the way many diseases and fevers are ushered in. If the chill be checked soon, this will stop, in many cases, the disease that was forming. For this purpose, give twenty lrops of the tincture of aconite root in a wine-glassful of water, and pour down the throat, out of a short-neck bottle; cover the body with a blanket, and rub the legs to bring the circulation to the surface of the body, and all will be well.

Choking.-Choking very rarely occurs in horses; very frequently in cattle. If choking should occur in the horse, there is little chance or hope of saving his life, if he be a spirited animal, and the substance be high in 
the gullet. In a very extensive practice, embracing many years, I have never seen a case of choking in the horse, except on a few occasions, and then it was only a ball of aloes sticking in the throat. If a ball of any thickness stick in its passage to the stomach, and it have passed down some distance, it is called low choke, and is not so . dangerous as if it stick in the entrance to the gullethigh choke.

Treatment.-In low choke, press down with the hand over the substance in the gullet, and try to move it. Do this not too strongly, but continue it for a time. If unsuccessful, one pint of fish, sweet, or linseed oil, melted lard, or sirup of any kind, will be apt to move the substance on its way down. If these should fail after a good trial, then have the gulles opened right over the substance, and take it out, and put in one, two, or three stitches, with strong saddlers' silk. Make the stitches separately from each other; for if this be not done, and one break, the others will also become loose.

In high choke, the irritation and excitement are great, which prevent much being done to relieve the animal. Try the oil, and see what can be done in that way. The treatment is purely mechanical, so use ingenuity to overcome the difficulty.

Chorea.-This is a rare disease in the horse, but common in dogs. It accompanies cases of madness in all animals, and depends upon nervous excitement, which is seen in the constant twitching of the muscles of the body. It is clearly sympathetic in its character.

Treatment. - Removal of the cause, whatever that may be.

\section{Cold.}

Symptoms.-Cough slight, fever, and discharge more 
or less from one or both nostrils, sometimes of a thin, watery material, or a thick, creamy pus. The lining membrane of the nose is red and inflamed. Cold sometimes even extends into the throat and lungs, giving rise to quickened breathing and uneasiness. When it extends to the stomach, it is called by the French gastritis mucosa. When it spreads to the chest, it is called bronchitis, (which see.) No disease is more common than cold among young horses; but, unfortunately, it rarely runs its course as such. Cold assumes one or more of the forms just mentioned.

Cause.-Exposure to cold and stormy weather.

Treatment.-In Germany, it is said that a cold, if let alone, will get well in a fortnight, and if treated by a skilful doctor, he will cure it in fourteen days. However, give the animal a few doses of aconite, (see Medicines,) to remove the fever, and, if possible, to prevent complications, or its farther spread to the neighboring parts. If the appetite keep good, nothing more need be done; but, on the contrary, if the breathing quicken, and the appetite be poor, and debility be setting in, tonics and stimulants will be necessary. Get the following medicine, and give one powder, morning, hoon, and night, mixed with a little cold water, and drench the horse with it:-Take powdered gentian root, powdered pimenta berries, powdered carbonate of ammonia, of each two ounces. Mix, and divide into twelve powders. When the appetite improves, give good feed, but not by any means in sufficient quantity to bring on indigestion. Give green feed, if it can be had. This is a most simple and successful plan of treating common cold.

Cold Lotions. - These are now called refrigerant lotions. Ice-water makes a good and economical refrige- 
rant, when applied to a sprain. (See Prescriptions and Medicines.)

\section{Colic.}

(1.) Spasmodic Colic.

Symptoms.-All at once the horse that a few moments ago was well, apparently, shakes his head-leares his feed, looks round at his flank, mostly at the right side, as if pointing out the seat of the disease, scrapes the ground with his front foot, and almost strikes his belly with one of the hind ones. The spasm continuing, the horse breaks out into a sweat, heaving at the flanks; great excitement, kicking, and rolling; intervals of ease from pain.

Causes.-Drinking cold water when heated, or colder water than commonly used, as a city horse is used to drinking water which is warmer in. summer, and colder in winter, than water taken from a pump; washing the belly with cold water; driving horses into a pond of cold water.

Treatment.-Give something to heat the stomach and bowels. Try a bottle of warm ale or porter, adding a little whiskey, or a tablespoonful of ground ginger to it. If relief be not obtained in half an hour, give a drench, composed of tincture of aconite root, twenty-five drops; spirit of turpentine, one ounce; one bottle of cold ale or porter. If necessary, give injections of warm water, (not hot,) soap, and a handful of table salt. Occasionally walk the horse about, to excite the bowels to action.

(2.) Stercoral Colic.

Symptoms.-Similar to the above variety, but continues longer, and is not quite so severe or painful.

Causes.-Impaction or constipation of the bowels.

Treatment.-Powdered aloes, one ounce; tincture of aconite root, twenty-five drops; chloroform, half an ounce. 
Mix in a bottle of ale or porter, and give in a drench out of a horn, or stout bottle. The aconite will have to be given every four hours, till the pain has given way. Encourage the operation of the aloes by injections every hour.

\section{(3.) Flatulent Colic.}

Symptoms.-Pain is considerable at first, which in a few hours gives way to sleepiness, (see Coma.) This is caused by distention of the bowels with gas, commonly called wind, thereby paralyzing the par vagus and nerve centres, and ultimately the brain itself. This variety of colic is readily distinguished from the others by the swelling of the belly, particularly at the flanks, called tympanitis, or drum-belly.

Causes.-Indigestion of food in the stomach; fermentation is set up, and there is evolution (giving forth) of carbonic acid gas. This gas is not liberated per rectum from the body as speedily as generated. The horse and cow cannot belch or eruct wind from their stomachs, as man and the dog can; hence the frequency of tympanitis in horses and cows.

Treatment.-Try injections first, as in many cases I have cured this variety by this means alone. If gas or wind come away with the injection, the case will soon end well. When no benefit is derived from the injections, give, in a little cold water, aloes in powder, one ounce; sulphuric ether, one ounce; tincture of opium, two ounces. If these measures fail in giving ease from pain, pour two ounces of chloroform on a small moist sponge, and hold it to the nostrils, not too closely, but admit a portion of air with the fumes of the chloroform, (see Medicines.) The sponge may be placed in a towel, and the ends car- 
ried up around the nose of the horse, to save waste; or place the sponge in the bottom of a nose bag, and put it on the head, but not too close upon the nose.

Last Remedy.-It must be confessed that the longer this disease is unrelieved, the more remote is the chance of recovery, as the bowel sometimes contracts upon itself, or nearly closes altogether. To overcome this condition an operation is recommended, which I do not say will be a success, nor yet a failure. When it failed in my hands, it was not because the gas was not let out of the bowels, but because the blood had become so disintegrated, and the nervous centres so paralyzed, that the sanative powers of the constitution had received too great a shock to ever rally again.

The Operation.-Procure an instrument, (see Instruments, ) called a trocar. If this be not at hand, sharpen a breakfast knife, and measure an equal distance from the haunch bone and the short rib, and not too high upon the back; force the knife into the distended bowel, and turn the knife in the wound thus made, and hold it there until all the imprisoned gas has escaped; and as the gas sometimes still accumulates, keep the knife or instrument in the wound, if it be for half a day. When the knife or instrument is taken out, place a piece of sticking plaster over the wound. (See Medicines.)

Stones, or hair calculi, are often found, after death, in the bowels of horses subject to colic. I have seen six taken from one mare that I had under treatment. Remember, the great principle in the treatment of colic, in all its forms, is to relieve pain. This also holds good in most diseases of horses. The doctor, if he fail to allay pain, cannot cure the disease.

Coma.-A horse is said to be in a comatose state when 
in an apoplectic fit, in sleepy staggers, and when dying from spasmodic colic, (which see.)

Congestion of the Lungs.-(See Lung Diseases.)

Constipation.-A confined condition of the bowels accompanying fever, liver and lung diseases. Horses habitually costive should be supplied with soft feed and grass in season. Costiveness, as a concomitant of fever, etc., should in all cases be let alone, as it is a provision of nature to protect herself from exhaustion.

\section{Consumption.}

Causes.-Repeated attacks of influenza, lung fever, or bronchitis, or any of these diseases treated by bleeding, and other reducing remedies or agents. Consumption, in the horse, runs its course in from one to two weeks.

Nature of Consumption.-A wasting, or breaking down of the structure of the lungs. The tuberculous form of consumption I have never seen in the horse. Tubercles are seen in bad cases of glanders, (which see.) These tubercles suppurate, and discharge pus. This pus is absorbed and taken into the blood, and sets up a ferment, or leaven, as the Scriptures call it. This is the great trouble in consumption in man, and glanders in the horse. Why authors have not called this tuberculous disease in horses consumption I do not know, except it be, and I am inclined to believe it is, from an entire ignorance of its true pathology or character.

Treatment.-Incurable. To prolong the life, beef soup, iron, gentian, tonics and stimulants, are indicated. In the tuberculous form, sulphite of soda should be given to arrest the ferment, and keep the blood clear of impurities, in addition to the remedies to support the strength. 
(See Medicines.) Decay, putrefaction, fermentation, are true and scientific expressions, with no vague meaning. Such, then, are the conditions embraced in the word consumption, whether in men or animals.

Contagion.-This term is applied to something, as the virus of glanders, coming in contact with the body of an animal in health, producing a similar disease to that existing in the animal from which it came. In a word, it is a specific poison. Few diseases of animals are considered contagious. The following diseases, however, are considered of that character:-Glanders, in horses; contagious typhus (rinderpest) and small-pox, in cattle; and small-pox, in sheep. Although many animals may be taken sick one after another, this is no proof that the disease (excepting those just named) is contagious; for it must be remembered that a number of animals, all situated and cared for in the same way, are certainly subjected to the same exciting causes that produced the disease in the first animal that was affected. Those that escaped the disease were not predisposed to take it; hence their exemption from its effects.

Prevention.-Separate the sick from the well. All buckets, or other materials, that were in contact with the sick, will have to be cleansed and purified. (See Disinfectants.)

Contraction.-(See Foot Diseases.)

Convalescence. $-A$ term applied to the time which elapses between the controlling of acute disease, and the restoration of the patient to perfect health.

Corns.-(See Foot Diseases.)

Coryza Gangrenosa.-This name is applied to an 
ulcerated condition of the membrane of the nose supervening in a case of cold. It is evidence of general debility, and disintegration or deterioration of the membrane.

Corrosive Sublimate.-(See Medicines and Poisons.)

(1.) Cough.-A symptom of disease of the respiratory organs, as tubercles of the lungs, thickening of the lining membranes of the windpipe, and enlargement of the glands of the neck. I have seen cough from indigestion in a few cases, but this is rare in horses.

\section{(2.) Cough, Chronic.}

Causes. - Debility, or softening of the par vagus nerves, heaves, or broken-wind, (which see,) are some of the causes which produce acute cough.

Treatment.-Removal of whatever is the cause, (causa sublata tulitur effectus.)

Counter-Irritants.-An external application, which, when applied, causes an irritation or inflammation counter or opposite to that which exists inside. (See Medicines.)

Cow Hock.-This name is applied to a condition or malformation of those hocks that incline forward, thus forming a fulcrum, over which the posterior straight ligament passes; this condition favoring a sprain of the ligament, which is called curb. (See Hock Diseases.)

(1.) Cramp.-Cramp of the stomach or bowels of horses, cannot readily be distinguished from spasmodic colic, (which see.)

(2.) Cramp of the Hind Leg.-This affection is common among young horses of an irritable temperament. It is often mistaken for dislocation of the patella, sometimes called luxation of the patella, (which see.) 
Symptoms.-The horse will persistently refuse to move the leg from the position in which it is placed. Quivering or excitement of the muscles of the thigh, accompanied with irritation and fever. The horse cannot be moved, as he refuses to do so.

Causes.-Irregularity of the nervous system.

Treatment.-Move the animal, if it be possible, and the cramp will give way. Dashing cold water against the thigh will often remove it. The horse will get well, if time be only given him. Thus, if a person leave the stable to tell some one of the matter, he will be surprised, on coming back, to find the horse well.

Crib-Biting.-This is not a disease, but a vice-a bad habit, which the horse has learned, of sucking wind into the stomach by placing his lips against the manger. The habit has been so strong in some horses, that when they could get no place to press the lips against, they have stooped down and placed the lips against the arm of their own front leg. This vice is sometimes called wind-sucking.

Causes.-Idleness, indigestion, and learning it from other animals in the same stable.

Prevention.-Keep horses in loose boxes, or other places where there are no fixtures but the walls; regular feed and regular work.

Treatment.-Do not let the horse stand in the stable twenty hours out of the twenty-four. Feed him regularly, and work him as regularly. Turn the animal to pasture, and when he is brought home in the fall of the year, have a loose box prepared for him without any fixtures, as manger trough or rack. Place his hay upon the floor, and his oats or corn in a small trough, and remove it as soon as the feed is eaten. 
Curb.-One of the many diseases of the hock-joint. (See Hock Diseases.)

\section{Curby-Hocked.-(See Cow Hock.)}

\section{Cutaneous Diseases.-(See Skin Diseases.)}

\section{Cutting.-(See Interfering.)}

Death.-The great law of the universe makes limits of duration to every structure endued with life, and prepares a way for the resolution of every material provided with vital principles into matter of the earth. The individual existence of all organized bodies is merely temporary; none escape the necessity of perishing.

Debility.-This is a condition accompanying many diseases. Hence, the necessity of guarding against any measure in the treatment, even of a disease of an exalted kind, that will reduce the strength. The animal thus affected rapidly becomes weak and debilitated to such an extent as may cost it its life.

Simple Debility.-May be local, that is, confined to a part, as in partial paralysis, from the effects of a blister applied to a part, or from a kick or injury.

Symptoms.-A thickening or swelling of the parts. The swelling is not inflammatory, but soft, and contains fluid. (Edema.)

Treatment.-Powdered sulphate of iron, one ounce and a half; gentian root, two ounces; chlorate or nitrate of potassa, one ounce. Mix, and divide into twelve powders, and give one night and morning mixed in cut or soft feed, with no more water in the feed than will keep the particles together. Feed the animal generously and well. Debility, and swelling of the legs of horses 


\section{DEFOR NITIES.}

now-a-days, at least in the United States, can be produced by simply keeping a portion of their usual feed from them for two or three days.

\section{General Debility.}

Symptoms.-Swelling of the legs, sheath, breast, and belly. Disturbed breathing. The horse is very weak, and easily pushed from off his feet by the hand. Inueed, he staggers as he walks, and sometimes falls to the ground.

Causes.-Bleeding or giving physic (a purge) in the treatment of disease, especially in diseases of the chest. Starving and low diet given to a sick horse, when he should be supported in the midst of his sickness by good feeding. The very neglect, or want of knowledge on this point, has killed thousands of horses that would have lived, that have been sacrificed to the shrine of ignorance, error, and bad judgment. This condition of things in relation to the treatment of diseases of the horse, and indeed all our faithful animals, may be ascribed to the diligence and persistence of the American publishers deluging the country with reprints of old English books that should never have been written.

Treatment.-Give plenty of good feed to the maltreated animal, and give the following blood-making medicines:-Powdered sulphate of iron, three ounces; gentian root, three ounces. Mix, and divide into twentyfour powders, and give one powder night and morning. If there is much swe about the body, add five grains of powdered Spanish fly to the evening powder for a few nights only. Be assured the animal is fully restored before it is put to hard work.

Deformities.-In young colts will often be observed 
a deformity of one or both forelegs from the knee down, giving the whole appearance an awkward look. Farmers having such a colt should not think it useless, or at all impaired; for in my own extensive practice, I have not seen one that did not become as perfect as the most perfect of animals.

Observe.-Whether the mother have sufficient milk for the colt, and if not, teach the colt to suck milk out of a bottle, or from something else; for by good nourishment these deformed conditions of the legs will disappear.

Diabetes.-This is a disease accompanied by a great and frequent flow of urine, containing sugar in solution.

Causes.-The sugar, starch, and some of the food are transferred into sugar. The origin of this affection is supposed to be a ferment changing the material of the system into sugar, as diastase converts starch into sugar in malting.

Symptoms.-Great flow of clear urine, very great thirst, ravenous appetite, weakness and general debility.

Treatment.-Although this disease is considered incurable, I have on two occasions cured the animal by the iodide of iron, too expensive a medicine to be recommended for general use. The action of this medicine, at least the iodide portion, allayed the thirst, and the iron supported the strength and system, also acting as an astringent (to dry up.)

This medicine should be seconded by good feeding and plenty of it. (See Iodide of Iron in Medicine list.)

Diaphoretics.-This term is applied to medicines having the power of producing sweating. The horse is not easily acted upon in this way by the use of medicines, except by one or two atricles, as aconite or vera- 
trum, (which see.) Slight moisture on the skin is very desirable in cases of fever. The cold water douche is used by hydropaths for this purpose.

When sweating is desired for the cure of disease, it must be done without increasing the heart's action. This is the great secret in producing diaphoresis.

\section{Diarrhœa.}

(1.) Simple Diarrhea consists of a looseness, or fluid condition of excrement, from something irritant in the bowels, and which does not freely pass away. When this condition is present, and no pain, griping or pawing as in colic, it may be well let alone.

(2.) Continued Diarrhea.-This is often the case when irritation does not pass away with the offending matter, and the bowels continue to discharge a thin fluid. There is slight pain or colic. When this is the casc, suspect some irritant poison. (See Poison.)

Treatment. - From whatever cause the diarrhoa may arise, treatment that will allay pain is demanded. First. Give twenty to twenty-five drops of the tincture of aconite root in a little cold water. Then give the following powder every two hours, until a change for the better has taken place:-Prepared chalk, half an ounce; catechu in powder, one drachm; opium in powder, ten grains. Allow the animal plenty of water to drink, which will help to keep or allay irritation, or what disposition there may be to inflammation. Bran mashes should be given for a few days, so as to overcome the effects of so much drying or binding medicine. Cake meal, or ground flaxseed, will be an excellent assistant in this particular.

Diathesis. - When we read in medical books of the 4 
word diathesis, it means a well-marked tendency in a disease by a corresponding diathesis: for example, a cancerous diathesis or an inflammatory diathesis.

Diet.-The different articles used by the horse as food.

\section{Disinfectants.}

French, Delsenfectants; German, Faülnisswidrige substanzen.

Disinfectants are such as remove the causes of infection, or any injurious taint. To accomplish this effect, disinfectants will have to embrace a class of substances known by the name of antiseptics, (agents which prevent animal or vegetable matter being decomposed,) and deodorizers, (agents which destroy hurtful or bad smells, when arising from decomposing material.)

(1.) Natural Disinfectants. - The atmospheric is the great disinfectant. The soil has been found a valuable disinfectant, decomposing animal matter with great quickness, and sending out gases which are without taint of any kind. Hence, the necessity of deep burial of animals dying, or that have been killed, on account of contagious diseases.

Ventilation is entirely a mechanical plan of disinfecting, and which is in the power of every farmer in the land to more or less perfect in all buildings containing horses, cattle, etc. In ventilating, it is only necessary to admit the purest air, and for this purpose have the openings, or ventilators placed at least eight to ten feet from the ground, as it is well known that heavy vapors are sometimes seen a few feet above the ground. Also, the ventilation should come from the front or top of the building, as the back of a stable is never so pure as its front. 
Water is the next great disinfectant employed by nature, although moist bodies decay more rapidly than dry. It is a disinfectant by the process of washing, which is mechanical. It is in this way that each shower of rain becomes a natural disinfectant. Light is another disinfectant, which seems to have been overlooked by many, when their barns and stables were built. Without light, the rose would lose its color, and man and animals would lose and never attain their vigor.

In proof of the advantage of light, in maintaining health and warding off disease, it is stated that in a barrack at St. Petersburg, there was only one case of disease on the side laid open to the light, to three on the dark side.

Heat and cold are two agents highly useful as disinfectants. Heat prevents fermentation and decay by drying and changing the chemical state of substances, as it were, by cooking, whether by fire or the sun. Cold, again, is the most powerful antiseptic and disinfectant. Frosts prevent decay and disease, and at the same time share the connection existing between them.

1. Artificial Disinfectants.-Creosote is a most powerful antiseptic and disinfectant, when applied to a part, but it is not easily managed. Smoke is another good antiseptic, as it contains a little creosote. By it herring and other fish are preserved.

Spices, and other aromatic substances, have long been used as "disinfectants, but they possess no such property, as they do not prevent decomposition of bodies: they merely cover the smell.

Chloride of lime and chloride of zinc act as good disinfectants.

The chloride of manganese is certainly as economical a 
disinfectant as can be used by the farmer. It is cheap and efficient, and not dangerous like chloride of zinc.

Sulphurous acid, or rather its fumes, has, in all ages, been used as a disinfectant, and by general consent it is considered to be most valuable. Its action on animal and vegetable substances is readily seen by the change in color produced. In the form of sulphate of soda, it will arrest the vinous fermentation in cider and other materials; or if injected into the veins of dead animals, it embalms them most perfectly.

For stables and houses filled with animals nothing will answer so well as chloride of lime, or McDougal's disinfecting powders, applied to the floors and excrement once per day with a large dredging box.

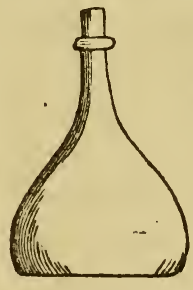

FLORENCE FLASK, used in generating chlorine gas.

For empty houses chlorine gas will be found as convenient and good as any. For this purpose, procure a strong widemouthed bottle, fill it about half full of bin oxide of manganese, close all the doors and windows, and other open places, then fill up the bottle with the spirits of salt, and retire and close the door. This may be repeated a few times in the course of a week. The fumes that are disengaged will penetrate to every crevice and corner in the building. This operation any farmer can perform himself, as there is no risk whatever. The spirits of salt will have to be kept in a glass-stoppered bottle till it is wanted, as it will not only eat a common cork, but it will, by exposure to the air, abstract moisture from it, by which it greatly loses its virtue and strength. As before stated, this plan of disinfecting is only to be used when the house is empty. (See Sulphurous Acid Gas in Part II.) 
DROPSIES.

Distemper.-(See Influenza.)

Diuretics.-A name given to those medicines which cause an increased secretion from the kidneys. Example, chlorate and nitrate of potassa, the Spanish fly, and eupurpurin. (See Medicines.)

Dropsies.-Every school boy is familiar with the term dropsy, which means an unnatural accumulation of water in the cavities of the body-chest, heart-case, belly, breast, sheath, and cellular tissue of the legs. (See Debility.)

1. When water is in the chest it is called Hydrothorax. This is the immediate cause of death, in pleurisy in the horse and pleura pneumonia in cattle, the animal dying by suffocation or asphyxia.

2. When in the belly, it is called Ascites.

3. When in the cellular tissue, and confined to a portion only of the body, as the leg or sheath, it is called EDEma; but if the swellings are over different portions of the body, it is called ANASARCA.

4. When in the heart-case or pericardium, it is called DROPSY of the IIEART.

Causes.-Treatment of diseases by starving, or low diet, bleeding, blistering, and physicking. Injury to a part will be followed by watery swellings in the neighboring parts.

Treatment.-Blood-making food and medicines, are imperatively demanded. Corn-meal mixed with bran and cut hay. Grass, if it can be had. A bottle of strong beef tea or soup, given daily, will be of great use. Give the following medicine three times a day, either mixed in the feed or poured down the mouth with a bottle :Powdered sulphate of iron, one dram; powdered gentian root, two drams; Spanish fly, two grains; mix. Friction 
over the swelling will be of use. Sometimes it will be necessary to make slight incisions or cuts through the skin to let out the imprisoned fluid. Do not blister such swelling, as it is apt to produce ragged, running sores, difficult to heal, and leaving a blemish.

Water in the chest is removed by introducing an instrument (See Instruments, ) called a trocar, but soinewhat smaller than the one used for hoven in cattle. Also it is removed by absorption and by diuretics. (Which see.)

\section{Dysentery.-(See Diarrhœa.)}

\section{Ear Diseases.}

(1.) Small Tumors.- Sometimes tumors of various shapes and sizes are seen in the ear of the horse, producing a kind of canker in that organ.

Symptoms.-Shaking of the head; will not let much familiarity be made with it; running or starting back, when the collar or bridle is being taken up over the ears.

Causes.-Irritation and inflammation of the skin of the ear, producing small pimples of proud flesh.

Treatment.-Removal with the knife, scissors, or caustic; then apply the simple ointment as for a simple sore. (See Prescription in Medicine list.)

(2.) Injuries of the Ear.-Injuries to the ear take place from the use of the whip, the bite of a dog, or from another horse biting it.

Treatment.-Treat as for a common sore, by simple ointment and by cleanliness.

(3.) Deafness.-Not often observed in horses.

(4.) Foreign Substances in the Ear.-Remove them by the forceps. 
(5.) Abscess in the Ear.-Treatment as for Abscess, which see.)

(6.) Dry Gangrene in the Ear.-Two cases of this affection have been brought to my notice, in which the concha of the ear had dried up, withered, and dropped off.

Ecchymosis.-Black spots observed on the lungs of cattle and horses having died from pleuro-pneumonia.

Eczema.-(See Mange.)

Elephantiasis. -A name applied to a swelled leg. (See Grease.)

Embrocation.-A term applied to liniments. (See Prescriptions.)

Emetics.-Medicine, when taken into the stomach of man and some animals, causing them to vomit. The horse, ox, and sheep do not, or rather cannot vomit. Hence, tartar emetic has no effect upon them.

Emphysema.-This is a name signifying wind swelling, caused by the escape of air into the cellular tissue, as between the skin and the flesh, or rather the fascia and the skin. Pressure with the hand on these windy swellings causes a crackling noise or sound. This condition sometimes arises in the chest, and is attended with difficult breathing and anxiety of countenance.

Causes. - Wounds in the chest, and when underneath the skin, it is caused by undue pressure on the part, causing a separation of the skin from the fascia.

Treatment.-This consists in evacuating the air by slight punctures on the surface of the swelling, assisted by gentle pressure of the hand.

When in the lungs, give good feeding, and occasionally small doses of the tincture of aconite root, say ten drops, 
three times in the day, to relieve the breathing, until the lungs heal up.

Encysted Tumors.-(See Tumors.)

Encephaloid.-(See T'umors.)

Enema.-(See Injection.)

Enteritis.-(See Bowel Diseases.)

Enzootic is a good name for cattle disease, as it makes the disease among animals instead of upon man.

Ephemeral. - A fever, which runs its course in twelve to forty-eight hours.

Epidemic.-This term is applied to diseases of animals, but improperly, as the term is exclusively a name that should only be used for diseases when man is the subject. Epi, upon, and demo, the people-a disease upon the people. Epizootic is the proper name for animal diseases. Epi, upon, and zoon, an animal-diseases upon animals.

Epizootic.-A disease that attacks many animals at the same time and season, originating in one common cause. Examples-epizootic-influenza in the horse, and pleuro-pneumonia in cattle.

Epilepsy.-An epileptic horse should never be used for family driving. (See Megrims.)

Epiphora.-(See Eye Diseases.)

Epsom Salts.-(See Medicines.)

Eruptions.-Eruptions are more a symptom than a disease itself. (See Strangles, Surfeit, Stings of Insects, and Scarlatina.)

Erysepilas.-This, as an independent disease, is not often, if at all, seen in horses. It is often present after accidents, as a bruise or a broken bone. 
Symptoms.-A hard, tense and painful swelling of the parts, accompanied with irritation and excitement through the entire system.

Treatment.-Give twenty to twenty-five drops of the tincture of aconite root four times in the day, to calm the system. Apply cold iced water, with woollen cloths, to the part. If, from broken bones, try and remove, or adjust them in position to one another, and secure them so with splint and bandage. In severe cases of broken bones, the animal had better be killed. (See Fractures.)

Exostosis.-(See Splint and Bony Tumors.)

\section{Eyes, Diseases of the.}

Before referring to the diseases of this organ, it may be necessary to say a few words as to the structure of the eye.

(1.) The eyelids are composed of skin, and under it the fibres of a circular muscle which close the eyelids. The eyelids are lined internally with a mucous membrane called the conjunctiva, which is reflected from them over the anterior surface of the eyeball.

(2.) The globe of the eye is not exactly round, for it is more like a segment of a smaller circle put into a greater one.

(3.) The clear portion of the eye is called the cornea, and forms a portion of the globe.

(4.) The sclerotic coat is what is called the white of the eye; and just back of it is what is called the choride coat of the eye. And immediately within this choride coat is placed the retina, or the expansion of the optic nerve, or the nerve of sight.

(5.) The humors of the eye occupy the anterior chamber, as well as pass into the posterior. The iris floats in this humor, and behind it will be found the magnifying 
lens of the eye. This is a double convex, and is placed in the centre of vision, and fronting the next, or vitreous humor of the eye. This humor is enclosed in a capsule, called the hyaloid membrane. The eyes of the horse are so placed in his head, that he, at the same time, sees different objects with each eye, which peculiarity accounts for the shying and starting, when one eye is imperfect or diseased. If one eye only be altogether destroyed, the horse will not shy or start, but will be a useful animal. This is the reason why cataract on the eye of a horse had better be let alone, as any imperfect sight will make the horse dangerous to drive, objects being seen in distorted form. Better the eye be completely destroyed than partially so.

(1.) Amaurosis.-This is the same disease that Milton was affected with, and was called by him "drop serene." It is a complete or partial blindness from loss of sensation, or feeling of the optic nerve.

Symptoms.-This is the most serious disease affecting the eye of either man or horse, and is ushered in by weeping and partial closure of the eyelids. A thin film or scum will soon be observed to spread over the whole of the front of the eye. This condition will pass off for a week or two, and then return with increased violence until the sight will be entirely destroyed. Spots will be seen between each attack, deep in the eye, gradually increasing in size, till the nerve of sight is totally destroyed.

Causes.-Inflammation affecting the brain, tumors, or bone pressing upon the optic nerve.

Treatment.-Doubtful of cure. Small doses of strychnine may be tried to restore the sensibility of the optic nerve, or colchicum may be used. (See Medicine.) 
Observe.-There are many horses with extreme flat foreheads, the bones, seemingly pressing upon the lower portion of the brain, just where the optic nerve is given out to the eyes. These flat-headed (that is in front of the head) horses, I have observed to go blind about the seventh year of their age. Blindness arising from such a cause, may justly be laid at the door of hereditary causes. Breeding from blind mares should, therefore, be, as a rule, avoided. If a mare from accident become blind, there are no scientific reasons why she should not be used for breeding purposes. What is to be observed is not to breed from a mare that has a very flat forehead, and has not gone blind from accident or injury to the eye.

(2.) Floating Spots in the Eye.-Sometimes dark cloudy spots or specks will be seen floating in the eye, more or less movable, rising and falling, as the eye itself moves. If they are unattended with weakness of the eye, or the spots are not fixed, and dark, indicating the commencement of the disease named above, they will be no great injury.

Causes.-Some constitutional disturbance, over driving, or hard work.

Treatment.-Inject, or apply cold water several times in the day.

(3.) Iritis.-Inflammation of the iris.

Causes.-Cold and exposure, producing rheumatism in that portion of the eye.

Symptoms.--Redness of the eye, and muddy color of the cornea. The iris is a little changed in color.

Treatment.-The application of moist poultices to the eye for a few days to allay inflammation, or irritation. Then apply three times daily, with a small brush called 
a camel's hair pencil, the following mixture:-Nitrate of silver, twelve grains; rain water, four ounces. The brush to be dipped in the mixture, and drawn lightly across within the eye. Keep the horse from the light as much as possible.

(4.) Inflammation of the Sclerotic Coat. Symptoms.-The coat is of a pink-red color.

Cause.-Rheumatism.

Treatment.-A teaspoonful of the wine of colchicum root, four times in the day; comfortable stabling and generous feeding.

Half-drachm doses of the iodide of potassium may be tried in this case. Half-ounce doses of sesquicarbonate of soda, occasionally, will be of service.

(5.) Polypi. Small excrescences are sometimes seen to arise from the iris, but require no treatment, as they will pass away of themselves.

(6.) Spots and Ulceration of the Cornea.

Symptoms.-Blood-vessels tinged with blood; small, elevated spots, which are sometimes ulcers, and at other times small abscesses, owing to the abscesses being whole or broken.

Treatment.-Take belladonna, half a drachm; cold water, six ounces. Mix, and apply to the parts with a camel's hair pencil dipped in the mixture. Scarify or open the angular veins of the eye, and foment with warm water, to insure a good flow of blood. This treatment should be followed by a lotion of the nitrate of silver, or of blue stone-sulphate of copper, (see Medicines,) to destroy the ulcers inside of the eyelids; then apply the belladonna lotion as before. It will require repeated applications alternately to effect a complete cure. It will be advisable to feed the horse well, to increase ab- 
sorption of effused fluids and thickenings. Iron and gentian will be useful. (See Medicines and Prescriptions.)

(7.) Weeping From the Eye.-This is more properly a symptom than a disease-a swelling of the caruncula lachrymalis-a small, round body.

Treatment.-Touch the swelling with a camel's hair pencil dipped in the lotion of blue stone, or nitrate of silver. Four grains to an ounce of rain water, will be strong enough for this purpose. This treatment will cure the weeping, provided the tear-duct be open.

(8.) Closing of the Eyelids.

Treatment.-Apply warm water with a sponge for a period sufficiently long, so as to dissolve or dilute the mucus, which causes the lids to stick together. To prevent a recurrence during some diseases of the eyes, smear the lids with sweet oil, or cold cream, every night while the disease lasts.

(9.) Hair Growing in the Eye.-Scientifically, this is called Trichiasis. The removal of the hair by tweezers, or forceps, and the application of some eye-wash to remove the irritation, are the proper means to be employed.

(10.) Swelling of the Eyelids.-This is sometimes observed in bad cases of mange, (which see.)

(11.) Simple Ophthalmia, or Catarrhal OphTHALMIA.

Symptoms.-A thick, mucous discharge from the eye, accompanying cases of cold or catarrh, redness and swelling of the membrane lining the inside of the eyelids. This condition and appearance resemble the lining of the nose in cases of cold. This affection may with propriety be called muco-purulent ophthalmia, and it is not unlike the Egyptian ophthalmia of man, produced by the intro- 
duction of the flying sands of the Arabian desert. Ophthalmia may be simple or acute; consequently, the treatment will be accordingly.

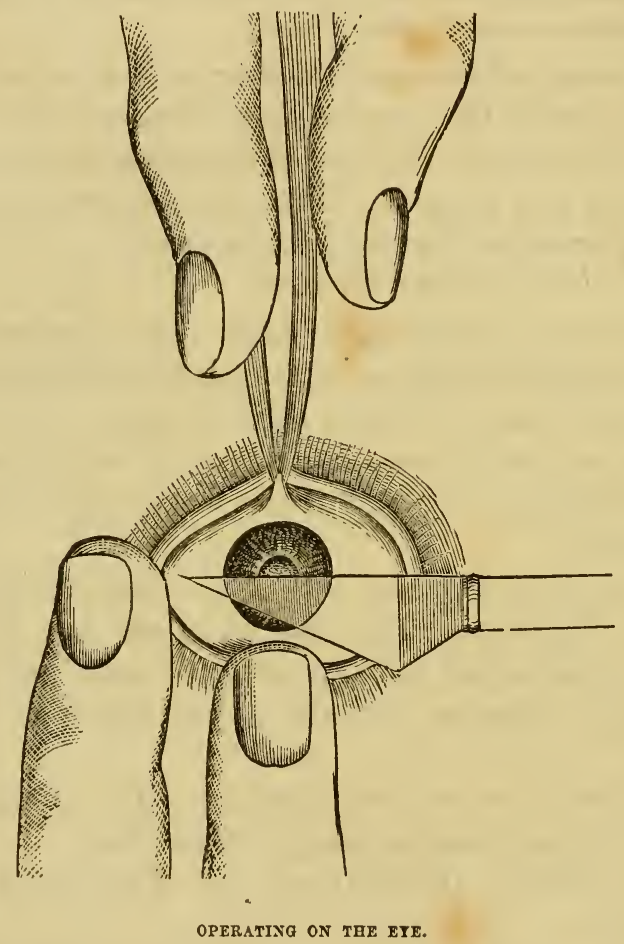

Treatment.-The application of cold water; or if the cold seem to give pain, warm water should be used instead. Open the angular veins. This is done as follows:-Take a sharp knife, and simply cut the skin and the veins which are seen underneath at the lower corner of the eye, and bathe with warm water, which will induce them to bleed freely. Give grass, or some other opening 
and cool feed. Give, also, half-ounce doses of sulphite of soda daily for a few days. The nitrate of silver lotion, or the blue stone, will do; it is cheap and easily procured, and therefore better adapted for the farmer or stable man. Apply as elsewhere recommended. Never use sugar of lead lotions as an eye-wash to the eye, which books so often recommend. They are positively injurious to the eye by their producing dulness, or opacity of the cornea-or the clear, transparent part of the eye-the very brightness of which indicates health, beauty, and intelligence in a horse; so, once for all, I say, use no Goulard's solution of lead. Copper is infinitely better, and never leaves dimness of the eye or vision. If copper be not at hand, chloride of zinc, one grain, to an ounce of rain or distilled water, is an excellent application to the eye of a horse suffering from purulent ophthalmia. (See Medicines and Prescriptions.)

(12.) Moon Blindness, terminating in Cataract.This disease is a serious one, and frequent, consisting of inflammation of the internal parts of the eyeball, the choroid coat and the iris more particularly.

Symptoms.-In the morning, perhaps, the eyelids will be found closed; a large flow of tears; the back portion of the eye dim and clouded. No specks are to be seen, as in some other diseases of the eye. A yellow border will be observed at the bottom of the chamber. This is pus. The attack, or inflammation, will last from two to three weeks; at the end of which time the eye will brighten up, and the ordinary observer may think that the eye is completely cured. The pus is entirely absorbed, scarcely leaving any traces behind, except a degree of dimness. In one, two, or three months, and not by the regularity 
of the moon's changes, as horsemen suppose, the disease reappears, having the same symptoms and characteristics as in the first attack, only a greater deposit of pus will be left at each subsequent attack. One attack succeeds another until the whole pupil is filled with matter, constituting cataract, thus completely destroying the eyesight. This disease is usually at first confined to one eye, but in some cases both are affected, one usually more severely than the other.

Treatment.-This disease is deemed incurable, which fact has often induced the owner of an animal thus affected to sell him, being well aware that the disease will at no distant day return, and leave upon his hands a blind horse. An application of cold water and the tincture of opium should be used to allay pain and irritation: cold water, one ounce; tincture of opium, two drachms; to be applied by means of a camel's hair pencil.

Observe.-When one eye of a horse is affected with this disease, the other will also ultimately become affected. To prevent this, it has been advised, as is done in man, to have the diseased eye entirely extirpated. In horses, I would rather puncture the cornea with a lancet, and allow the watery humor to escape, thus permitting the diseased eye to sink in the head. This being done successfully, the remaining eye will not only retain, but will increase in lustre and brightness.

\section{False Quarter.-(See Foot Diseases.)}

Farcy.-The reader will be not a little surprised at the opinions that are advanced by me in regard to this disease, especially if he be a reader of the books on the diseases of horses. In these books we are distinctly told that farcy is a variety of glanders, and that farcy buds 
are of the same nature as the ulcers of the lining membrane of the nose in cases of glanders. This may or may not be true. But why not have given the reasons why these relations were so related to each other? Thus assertion is put for fact, and ignorance for great knowledge. Farcy is not a disease attacking the absorbent vessels, nor glanders of the lining membranes of the nose. Farcy, we are again told, is curable, and in the very next sentence that glanders is incurable. Why this peculiarity? for if the diseases be the same, they ought to be both susceptible of cure. From all that has been said and written on the subject of farcy and glanders, nothing satisfactory has been gained, but much that is calculated to perplex. Farcy is the "scrofula" of the horse. It is unknown in countries and climes where this disease in man is never seen, and a disease inseparable from the present manner of domestication. In a word, the disease called farcy is nothing more nor less than the effects of a class of pathogens called ferments, leavens, or zumins, acting and producing fermentation in the blood. (See Glanders.) In medicines zumins are used, such as yeast, rennet, pepsin, and cow-pox matter. Among the various diseases of the horse produced by ferments, are glanders, farcy, purpura, grease, and several eruptions of the skin and legs. This is readily explained. For instance, if the liver, kidneys, skin, and bowels of a horse be not acting right, how is effete matter to be eliminated or carried from the blood or the body of the animal? This effete matter, as a small piece of membrane, dead bone or pus, not escaping by the usual channels, will decay and become an active ferment in the blood and in the fluids of the body. This, then, is the only true explanation of the phenomena of farcy in the horse. 
Symptoms.-An unhealthy coat; bad habit of body; one leg, usually the fore leg, will swell to a very large size; hot and painful, and in a day or two it will break out in small running ulcers, or sores, discharging a sanious fluid, sometimes of a thick and resinous color. On the inside of the leg, or on the side of the body or the neck, will be seen a thick, corded, and elevated substance under the skin, of considerable hardness, and interrupted at distances with a small sore similar to that on the leg. In some cases-not in every case-circumscribed, and soft, puffy swellings, will be seen about the mouth, lips, and indeed on many parts of the body. These swellings are not to be confounded with swelling of the legs, belly, breast, sheath, etc., in cases of weakness or debility. These swellings have been named water farcy by some people, but have no connection with true or malignant farcy whatever, and are not in any way infectious or contagious. After some alterations and changes occupying a few weeks, the animal becomes much changed for the worse, the blood becoming so deteriorated and changed in character that the animal's vitality soon gives way, and the horse dies a miserable object.

Causes.-Overcrowding horses in small and insufficient houses, with little or no ventilation, each animal repeatedly breathing the noxious or waste material from the lungs of his companions, thus introducing into the blood a powerful pathogen in the form of a ferment; inoculation from the virus of glandered or farcied horses, the inoculation producing a ferment; debility, ending in changing the character of the blood. Absorption of pus from sores or ulcers into the blood is another powerful ferment, and ends either in farcy, glanders, or some other zymotic disease. 
Treatment.-Having fully and sufficiently explained the cause of this disease, the treatment is almost already pointed out, namely, remove the cause, and the effects will cease. For this purpose give the following substance twice a day, a tablespoonful to a dose:-Sulphite (not sulphate) of soda. This new chemical salt will purify the blood, as its action destroys fermentation, whether in a barrel of wine, cider, or in the blood of a horse. Continuing this till the horse is well, and for a few weeks afterwards, two or three times a week, will be of good service. (See Sulphite of Soda.) While the blood is thus being purified and made fit once more for the purposes of life, it will be necessary to add something to it also, thus assisting the powers of the constitution to overcome the difficulty. We will not only add to the blood, but give something to facilitate the removal of the effete matter from the body, without weakening the animal with debilitating diuretics. For this purpose, the following medicine will be given:-Powdered sulphite of copper, three ounces; powdered Spanish fly, one drachm; powdered gentian root, four ounces. Mix, and divide into twelve powders, and give one powder at night in some good feed, with no more cold water in it than will keep the particles of the feed together. These powders will do for two weeks; at the end of that time get more, and continue them till the horse is well. In addition to what has been recommended, give grass, and good and generous feed, for by these means the power and strength of the body will be kept up, and a cure will be more surely and speedily effected.

While this treatment is going on, the horse will have to be removed to a place by himself, or from healthy animals; keep all articles used in feeding, clothing, and 
cleansing him, in the building with him. Remember, this is considered one of the contagious diseases of the horse. Not only the safety of your own, but of your neighbor's horse, depends upon your observance of these directions. In case you should place a horse so affected, either in a field next to your neighbor, or in his stable, and his horses become affected, you will be liable to a suit at law for damages arising therefrom. From my experience in horse causes in the courts of Philadelphia, it will be easier to bring a suit than to gain it, if there be scientific witnesses on the stand, as the question will arise, Was the disease, or was it not communicated from the sick horse so placed? Obviously, these are questions more easily asked than answered.

\section{Fatty Tumor. (See Tumors.)}

Farrier.-Properly, this title belongs to the blacksmith, whether a horse shoer, or of other branches of iron working, faber ferrarius; but from some idea or other, we hear of persons, otherwise well informed, saying, when speaking of accident or sickness to horses, to have or send for a farrier. Now, what is there possessed by workers in ,iron, that they should know any more about diseases and their treatment than is possessed by a worker in wood. Persons of education should look into the etymology of a term, even if it should be used by the mass of the people: for it is by the language and general deportment, that a man of education is known from his less learned prototype.

Fever, Sympathetic.-This variety of fever is that which is produced by accident and disease. Thus, a horse gets a nail in the sensitive part of the foot, excitement or sympathetic fever is an accompaniment. Lung 
disease is accompanied with fever, and hence it is called lung fever. Fever in the feet is a common expression, signifying sympathetic fever. Indeed, it is a question in my mind, whether fevers of all kinds are not to be attributed to some local or general disturbance of some action or function of the body. Thus, the many fevers which attack the human family, can readily be traced to a predisposing cause; as, for example, typhus fever is caused by insufficient ventilation, besiged towns and garrisons, preventing the removal of exuvia.

Fever in the Feet. -This is a common disease in horses of large towns and cities, where the streets are paved with stone or iron, whereby the concussion is very great, when horses are driven fast. (See Founder.)

Fibroma.-A variety of tumor. (See Tumors.)

Firing Horses.-This is an operation which is a great favorite, and in much repute among horse doctors. For my part, I think it not only cruel and barbarous, but unnecessary, doing no good whatever, but in many cases a positive injury, from which the effects will never disappear from the legs or body while the animal lives. Firing is intended by its advocates to prevent and cure spavin, curbs, sprains, and ring bones, by scoring the parts with a red hot iron in lines over the part that is thought will be diseased or are already so.

Fistula.-Examples.-Fistula of the shoulder, of the poll, poll evil, (which see,) and quittor or sinuses of the foot, (which see.) Fistulas are usually deep-seated, but sometimes they are superficial, or just under the skin. However, notwithstanding, that we see them sometimes so situated, it must be confessed that it is extremely rare. The fistula, most frequent and difficult of cure, is always 
deep-seated, and in the vicinity of a joint, as the poll and shoulder. The reason of this is easy of explanation, for when these parts get injured, and suppurative action is set up, the pus, instead of pointing to the outward surface, burrows down in among the loose textures, and forms sinuses or pipes, pointing in several directions. Fistula differs from a simple abscess only in this particular, and the difficulty of cure. The pus secreted is nearly the same. The pus in a simple abscess is secreted from, and a liquification of the surrounding tissue and the pus of fistula is secreted from the walls of a fibrous sack, which is formed in most cases of fistula. The fistula may be open or whole, presenting a large swelling.

Symptoms.-Pain on pressure to the parts affected, followed by heat, pain, and swelling, circumscribed in shape, hard at first, and becoming soft and fluctuating upon pressure by the finger, and sure indication of fluid within. This swelling, from the firmness and integrity of the sack and skin in which it is enclosed, does not break, nor yet form sinuses which take on the character of an encysted tumor, which does not break of its own accord, as simple abscesses do. At other times, the fistulous tumor breaks or opens in several places, and small holes discharge pus, some to-day and none to-morrow. The day the discharge is free, the pain and swelling is less. Among the pus will be observed organized matter like cheese, or in other words, it is not uniform in thickness or appearance. This is a disease which rarely ever gets well of its own efforts. This arises from the nature of the sore having a sack, which is only removed by art. Also, from the situation of fistula presenting no depending opening for the outlet of the pus.

Causes.-Bruises, accidents, caries of the bones, (which 
see,) inflammation of the bone, or any accident that will cause a simple abscess, will cause fistula.

A blow that would produce a common abscess on some portions of the body, will result in fistula in other portions, as in the vicinity of a joint.

Treatment.-If the swelling is just forming, endeavor to put it back by placing chopped ice in a bag over it for a few days. By this means, many a swelling which would terminate in a fistula, will be cured at once. This not succeeding, have an opening made into its lowest side, so as the bloody water, which it at this time contains, will be discharged. Then syringe a half table spoonful of the tincture of iodine into it once in the day for a few days, to eat or destroy the membranous sack. Then treat it as a common sore by keeping it clean, and the opening from closing before it has healed from the bottom. For this purpose, place a small piece of cotton in the mouth of the opening, smeared with simple ointment. (See Medicines and Prescriptions.)

Fistula in the Foot.-This disease, by common consent, is called quittor, a fistulous abscess in the foot. (See Quittor.)

Fistulous Withers.-By farmers called Thisolow, or Fisolow. This is an affection described in the preceding article with this difference in some cases, that it extends through over the top of the bones of the withers or shoulders to the other side, thus forming a very broad pad, as it were, on the top of the shoulder, just where the shoulder in health is the sharpest and narrowest.

Treatment.-Chopped ice in bags laid over the swelling, and, if necessary, open and take out the sack, or destroy it by the injection of the tincture of iodine, and 
dress as for a common sore. (See preceding Article and Poll Evil.

Fits.-This is a term applied to horses subject to megrim, or staggers, (which see,) causing them to fall. Hence, the expression, "fitty horse."

Flaxseed.-Every part of this seed is used one way or another in the treatment of diseases of the horse. The ground seed, mixed with warm water, is an excellent cooling food for horses, almost a laxative. The pressed juice, or oil, is a certain and safe purgative for the horse in quart doses. The residue, which remains after the oil has been pressed, commonly called cake meal, and when mixed with warm water, makes the best of poultices to a sore or wound. (See Poultices.)

Fomentations.-This term has been used exclusively to the application of warm water to an inflamed or sprained part, and sometimes to a sore. I may be right or may be wrong, when I say that the application of cold water to parts similarly affected, are just as much entitled to the term fomentation, for certainly it is applied the same way and for the same purpose, namely, to allay irritation in the sore or sprained part; and it has from my own experience, proved to have a much better effect, and in as short a time. Cold applied has an effect to brace, strengthen, and give tonicity to relaxed sprains and sores. Warmth has an opposite effect, . e., to relax and debilitate. In foot diseases, warm water will be preferable for softening the horn. Where warm water is used, the cure should be treated afterwards by cold fomentations. A good way to apply cold water is by means of wet woolen cloths wrapped loosely around the parts, and wet every hour or so before the skin becomes warm. At 
night take all the cloths off: this will prevent scalding, and falling off of the hair.

Warm water should be applied the same way as cold.

Either cold or warm fomentations should be continued for a time to get their benefits. It will be well to remember, that when the cloths are allowed to become dry, an opposite reaction is immediately set up. Cold is followed by increased warmth in the parts. Warmth is followed by cold. This should be well understood, for in my opinion cold applications, with absolute and entire rest, are the only and best agents for the speedy and sure cure of sprains in whatever part of the legs or body.

\section{Foot Diseases.}

(1.) Canker in the Foot.-Happily, this is a rare affection, because it is not easy managed from the peculiar tendency in the horse's foot to grow and produce proud flesh, of which this is the essential principle of the affection. Canker in the foot of a horse may be said to be a foot deprived of a part of its sole, and in its stead a fungus takes its place. Difficult to keep down level with the remaining portion of the sole. And not only that have we to change this disposition in the foot to throw out this material, but induce this material to secrete or produce a new sole. This is the difficulty to be experienced in the treatment of this affection. Nevertheless, cases, and very hard ones, too, have been cured, but not in a day, nor a week, but months.

Causes.-Injuries to the sensitive sole by nails, bruises, and other accidents, as a piece of the sole being torn off.

Treatment.-Removal of any diseased or dead sole, and the proud flesh. For this purpose, the knife will have to be used to remove the dead sole, and, if it be in 
the hands of an energetic person, the most of the fungus or proud flesh can be taken off in the same manner. If not, get caustic potash, and quickly reduce it to a coarse powder, as it soon dissolves on exposure to the air. Lay it upon the raw surface. This apply next day, if the first application has not removed sufficient or all of it. After the proud flesh has been entirely taken off, and level with the sole proper, dress every day with Barbadoes tar, one pound; sulphuric acid, three drachms; powdered sulphate of copper, half an ounce. Mix well, and spread a portion over the sore foot, and over this dressing, a pad of tow or cotton, held firmly down on the padding, so as to produce pressure, an important matter in the treatment of canker in the foot. This can be secured by thin pieces of splint from young wood, being placed across one another over the pad, and the ends pushed in between the foot and the shoe. By this means, and a little patience, and a little ingenuity in fixing and applying these pads, etc., even very bad cases can be cured.

(2.) Contraction.-This is not so much a disease as it is of bad management in the stable and in the blacksmith's shop. Contraction may be said to be an alteration of the shape and structure of the posterior, or back portion of the hoof-a winding in of the heels.

Causes.-Want of proper knowledge on the part of the owner or horse shoer in not knowing the difference between the foot that would require a piece added to it at each shoeing. and the one that requires a large portion taken from it, so as to insure elasticity and expansion. A foot strong and inelastic, and unyielding to the weight of the animal, is the very first foot to become contracted. I know flat, weak-footed horses travelling 
sound for ten or fifteen years without in the least being contracted. So long as we have strong huofed horses, and shod with an inflexible iron ring to prevent its wear and tear, and the blacksmith neglecting to have taken off of each hoof at the shoeing as much horn as the horse would have worn, if he had been in the natural state and not shod.

Treatment.-Cut down the hoof and shorten the toe, and make the hard and inelastic foot one that will expand at its heels every time the weight of the animal puts his foot to the ground.

One sided nailing is an excellent preventative as well as an assistant in the cure of contracted feet. What is meant by one sided nailing is, that nails are to be confined to the outside toe of the foot, so as to allow free expansion for the heels. This cannot be done with the shoe being nailed on by nails placed at each side. To illustrate this point, place a small horse shoe flat in the palm of the hand with the fingers close to one another; then tie with a piece of cord, the little finger to one side of the shoe, and the thumb to the other side; then you will realize to what extent you can expand the fingers so secured. So it is just with the foot of a horse with the shoe nailed on at each side. Remove the cord from one finger, and the whole hand is free to expand. So likewise the foot of the horse by one sided nailing.

(3.) Conss.-This is a red spot on the inner portion of the heel of the foot, causing lameness, and consists of a bruise from the shoe pressing upon the part, the shoes having shifted from their proper position, or never having been placed there. In general, the production of corns may be laid to the charge of the horse-shoer, and sometimes to the owner allowing the horse to go too 
long before the shoes are removed, or before the foot has grown from the shoes. Corn is an analogical term, although bearing no resemblance to that well known affection of the feet of man.

Treatment.-Remove the corns by cutting them out; then apply a few drops of commercial sulphuric acid to the part. Shoe the horse sufficiently often to insure even bearing to the shoe upon the wall only of the foot.

(4.) False Quarter.-This is a term applied to the horn or portion of the hoof, which overlaps or bulges out a little from the line of the sound portion of the hoof.

Causes.-An injury to the coronet or ligament, which secretes, or from which the hoof grows. This causes an alteration of the horn of the hoof below, and corresponding to the extent of the injury.

Treatment.-As there will be an enlargement, more or less, remaining after an injury to the coronet, little will be required to be done, but to keep the horn as level with the hoof proper as possible in order to make it look less of an eye-sore.

(5.) Inflamation of the Foot.-(See Founder.)

Pricks._-Pricks may occur in the act of shoeing, or from a nail being picked up on the street, and from other hard-pointed substances.

Treatment.-Pull the nail out, and poultice the foot for twenty-four hours; then make an opening through the horn over the place where the nail went in, so as to allow the pus to escape. For, if this be not done, and the pus is left in the foot, it will in a few days, and at a great expense of suffering to the horse, break out between the hoof and the hair, constituting quittor. After an opening has been properly made, drop five drops of 
muriatic acid into the hole once a day, for a day or two. Poultice every second night or day, and not oftener. A healthy hoof can be poulticed off by constant application.

The horse can go to work as soon as he can step on the ground firmly and without lameness. Remomber that in all cases of pricks and other injuries which end in suppuration as pus in the foot of a horse, making a thorough opening so as to allow the pus to escape, and no more trouble need he apprehend, except the nail has entered into one of the tendons of the foot, in that case no pus will be usually formed, the majority of cases ending in

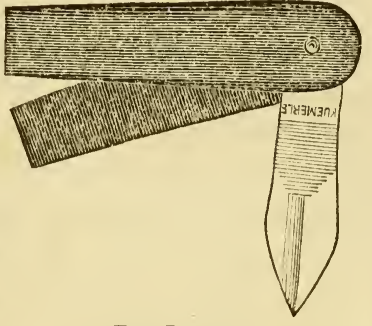

Foot Lancet. locked-jaw, (which see). Where pus is formed after pricking, no locked-jaw follows, it is in those cases only where pus is not formed that locked-jaw may be expected.

6. Bruises of the Foot.-(Treat as for prick in foot).

7. Sand or Quarter Crack.-This is a crack or split in the hoof, usually on the inner side of the fore foot, although splits and cracks occur in all places, and in both fore and hind feet.

Causes.-A brittle condition of the hoof, from the want of sufficient moisture. In some cases the horse does not stand square upon his fore feet and thereby causing undue weight to fall upon one of the sides of the hoof and causes it to crack.

Prevention.-Apply to brittle feet equal portions of the oil of tar, and cod liver oil, whale oil, or any fish oil well rubbed in with a brush to the hoofs a few times a week, and occasionally applying wet cloths to the feet 
in the summer season. In winter the feet are better supplied with moisture.

Treatment.--Rasp the edges of the crack thin, the nearer the crack the thinner the horn should be made, this can be filled up with shoemakers wax. After the horn has been properly thinned a piece of the wall of the hoof for about half an inch on each side of the crack is to be cut out to prevent any bearing of the shoe upon it, thereby preventing the constant opening, shutting, and sometimes bleeding from the crack. To prevent or rather to cause the new horn to grow down whole and without a crack in it, a piece of iron is to be placed in the fire and made red hot, and applying this just for a moment flat on the hair at the head of the crack sufficient to make a scab. This will insure a solid growth of horn. Promote the growth of the horn as speedily as possible, to facilitate the cure, a strap is used by some round the hoof to prevent opening of the crack.

8. Navicular Disease.-This is a disease of a bone in the foot of the horse, a serious disease and often very obscure in its symptoms, altogether depending as to the advanced condition of the disease, happily however it is scarcely met with and then only in the common hack horse. It consists of ulceration of various degrees; on the surface of the navicular bone within the foot.

Causes.-Hard and constant work upon the stone or streets paved with stones. Slight inflammation neglected and the horse not laid up until it gets well, and the inflammation increases, until it ends in ulceration of the bone.

Symptom.-Obscure, or at least very like many of the symptoms similar to other diseases of the feet and legs. It is chiefly determined by the undue heat in the foot, and 
by the fact that no diseases can be detected elsewhere or of a different kind. In lameness of other portion, not in the foot we have heat and swelling-the hoof or foot of the horse cannot swell. It is a box and we cannot see into it to examine its contents and condition.

Treatment.-Incurable, although much can be done to relieve the pain, by the application of moisture poultices, and occasionally placing the hoof in warm water to soften and relieve pressure. Some have recommended a blister round the coronet. I cannot see in what scientific, or other grounds it will do good. In well established cases, and in good strong feet the division of the nerves which run down on each side of the leg and into the foot, will relieve the pain and the horse will go well for years. But unfortunately many cases so operated upon have lost their hoofs by falling off which has been unjustly laid or blamed to the operation. The cause of this is not so much from the operation as from the operator having no judgement in the kind of hoof, the operation would be or not be successful. It would be obviously improper to recommend the operation upon a flat footed horse, as the animal deprived of pain would let his thin soled foot come down to the ground with great force and weight, thus injuring the whole structure to such an extent, that suppuration would take place and ultimately the sloughing and falling off of the hoof.

9. SeEdy Toe.-This is a name given to a split in the centre of either fore or hind foot, extending a little way up from the point, or it may be up to the hair itself.

Causes.-The same as those producing sand-crack. (which see).

Treatment.-In bad cases a clasp or plate of iron 
secured by short screws. In simple crack or split from the toe upwards, cut off all communication of the sound parts with the cracked or split portion. For this purpose a three edged file will be a good instrument for making the division. At each shoeing the split portion will gradually become less or shorter.

10. Pumrced Sole.-This name is used or applied to a sole of a foot, which is convex instead of concave, that is instead of the nice cuped foot, the bottom of the saucer is presented.

Causes.-The result of bad or severe causes of founder, where the coffin bone is let down upon the sole, and causes its descent or convexity.

Treatment.-This is merely palliative and this is to be done entirely by placing a shoe upon the foot, that will insure and protect the sole from the ground.

11. Thrush.-A diseased condition of the sensitive frog of the foot, and from which a stinking fluid is discharged, which is familiar to every body who is among horses.

Causes.-T'oo much moisture to the foot, as from animals standing in their own excretia, or from wet stabling, the frog becomes perverted, and detoriorated, and secretes, or discharges a blackish color, and otherwise nasty fluid. It sometimes accompanies navicular disease.

Prevention.-Dry stabling, a stall having sufficient inclination or drainage to carry of the fluids. Cleaning the stables regularly.

Treatment.-A few drops of muriatic acid forced into the centre of the frog once a day, for a few days. Keep the stable and stalls dry and clean. A few doses of the sulphite of soda in half ounce doses, once a day, 
for a few days, will do good by its alterative and puritive effects upon the system.

QUitTor IN THE Fоот.-This is a serious and painful disease of the foot, of the same nature as poll-evil and fistula in the shoulder, it is known by a large swelling around or above the hoof, or where the hair joins the hoof, which soon breaks and discharges pus.

Symptoms.-Horse off his feed. Considerable excitement and fever. Holding the foot off the ground, and very painful. A swelling soon shows itself at the coronet, and in a few days breaks and discharges pus. After this the horse will resume his feed, but will not make very free with his foot, which will be better to-day and worse the next day, depending upon the discharge of pus from the foot.

Causes.-A prick from a nail, a bruise on the sole, a suppurating corn, or sometimes from a sand crack taking on suppurative action.

Treatment.-The great secret of the treatment of this disease, is to make a free opening from the bottom of the foot. When this is done at once, you will be greatly surprised to see the swelling go away as quickly as it made its appearance, and if the swelling has broken, it will soon cease to discharge when the opening is made from below, or at the bottom of the foot. Having effected this purpose of an opening, get a small syringe and inject into the opening above, if there be any, and if nor from the opening below, a mixture of the sulphate of zinc, two ounces; rain water, eight ounces, once in the day. If the shoe has been taken off for the purpose of making the opening, have it put on again so as to hold some soft stopping in the sole to keep it soft. Cow dung is as good if not better for this purpose, than the best of 6 
costly flax seed. Never in cases of this disease, apply poultices around the whole of the hoof, as in that case the hoof niay fall off. Healthy hoofs can be poulticed off. Whatever poultice, it must be applied to the sole. If no opening has been made from below, drop 10 drops of muriatic acid into the opening above, once in the day for a few days. This will destroy the disease.

\section{Founder.}

(1.) Acute Founder.-Every school-boy is familiar with the name of founder when applied to a lame horse, but few horsemen ever comprehend the nature and seat of this affection. Founder in all its forms is inflammation of the laminæ or leaves, which dovetail into each other, and bind the sensible and insensible portions of the foot together. Hence, it is called by some learned persons in diseases of horses laminitis, by adding the Greek word itis. Founder is again called by some persons fever in the feet. However, as to names, the disease is of often occurrence, and when left to itself, it destroys many good horses by leaving them ever afterward sore and tender ir. front, as the horsemen have it.

Symptoms.-The horse will scarcely move; stands upon his heels, with fore feet and legs stretched out as far as he can get to throw the weight off them. Thus, to all appearances, making the animai hollow in the breast, which appearance has given rise to the idea that the horse is chest foundered. The hind legs are brought far in under the belly. The head of the horse is erect and high. Fever and constitutional disturbance are very great. The horse is extremely excitable, and breathing fast and laborious. Altogether, the poor suffering horse is the very picture of distress and disease. 
Cause.-Giving cold water when overheated, and tired from overwork. A tendency in the feet to take on inflammatory action. The animal not in proper health or condition for performing heavy or fast work.

Treatment.-Place the horse in a wide and airy stall, with plenty of good straw for bedding to encourage the horse to lie down, which will relieve him very much. Indeed, so much is this the case, that it has been recommended that every foundered horse should be forcibly thrown and kept down, till the active stage of the disease has passed off. This, however, I do not advise, as the horse is excited enough without increasing it by throwing him from his feet. Rather give good bedding, and the majority of horses so affected will be ready and willing to lie down of their own accord. After the place is all fixed, and the horse moved into it, give him twenty drops of the tincture of aconite root in a cupful of cold water poured into the mouth with a bottle having a strong neck. Repeat the dose every four hours, till six to eight doses have been given. Apply cold ice water cloths to the feet. In a few hours, possibly, the shoes can be taken off. At first, this generally cannot be done, except the animal is down. Care should be taken in removing the shoes, so that every nail is made loose before an attempt is made to pull off the shoes. Have as little hammering on the foot as possible, as it will shake the great and over-sensitive frame. Let the cold water be kept constant for the first day, or until the active pain gives way. At leisure, the feet can be pared thin on the soles, so they will yield to pressure with the fingers. By getting the animal to lie down as soon as possible after he gets in, the cold water cloths applied, and the aconite given, the animal in a day or two may 
be nothing the worse from the attack. The longer the animal remains before these remedies are applied, the less likely is he to be free from its effects afterwards. Before the horse is again put to work, be assured he has quite recovered. During the treatment, give plenty of cold water to drink. Never give tepid water to a horse while he is sick from disease. Give grass or soft mashes for a day or two, but do not keep a sick horse too long on low feed, as debility and swelling of the legs and various portions of the body will take place.

Remember.-Do not bleed, neither from the neck, nor foot, nor from any place else in a disease of this kind.

(2. Chronic Founder.-Symptoms, Cause and Treatment will be much the same, only it will not be necessary to push the treatment so far. Principally depend upon softening the horn of the feet, paring the soles of the feet, and a few days' rest. The horse, for a week or two afterwards, should be placed in a stall having six inches of sawdust spread over it, and kept a little moist with water poured over it once in a day. When horses are recovering from acute founder, they also might be placed in a stall so prepared. Clay stalls are objectionable.

\section{Fractures.}

Fractures.-This term signifies a broken bone. Fractures are the result of great force applied to the bone, as from kicks, falls, and accidents.

Fractures occur in three forms.

(1.) The simple fracture or break, either oblique, or slanting, or straight across the bone.

(2.) The compound fracture, where the bones are broken in several places.

(3.) The comminuted fracture, where the bones are 
broken in pieces, and the ends of the broken bones are seen cutting through the skin, and, possibly, severing some of the blood vessels, causing, in many cases, death from loss of blood.

The first, or simple fracture, is the only variety that calls for much notice, as the other forms of fracture, when occurring in horses, are beyond remedy. Simple fracture of the following named bones may, with care, be in a great measure cured, and the animal be made useful for many purposes. As, for instance, it would be obviously wrong to have a valuable mare destroyed, because of simple fracture of one of many bones, as she would be useful for breeding purposes, if not for the farm or the road.

Fracture of the bones of the leg any where from the elbow down, except it may be the knee joint, can readily, with a little care and attention, be secured in its place, and the animal be made useful. So also the hind legs from the hock down to the foot. Fracture of the ribs usually get well of themselves, because they are always kept in place. So also of the haunch bone. Indeed, simple fracture of most any of the long bones will unite of their own accord, if the bones are put and kept in their place by proper fixings.

Symptoms.-The symptoms of fracture of the long bones of the legs are seen at once by the looseness of the leg, the horse not having the p̀ower to move it. The leg can be turned in any direction. The horse is in great pain, excitement, sweating, etc. If there are bones pointing through the skin, the horse had better be shot.

Treatment.-Place the horse, if in summer season, in the field, and in winter in the barn-yard, where there is 
not too much straw to prevent the foot being carried without interruption.

Then give twenty-five drops of the tincture of aconite root every five hours, for the first twenty-four hours, to relieve pain, excitement, and fever. Take hold of the broken leg carefully, and place it in a position as near like the other leg as possible, not only in shape, but in actual length by measurement, for it is the neglect of this that some legs are left shorter and some longer after fractures. The whole position of the leg being got at, apply a good coating of tar to the leg, around, above, and below the point of fracture. Then wrap the whole leg in oakum, coarse tow, or carded cotton. Over this, place broad pieces of boards, like shingles, on the sides and front of the leg, and fill all uneaveness with the tow or cotton, so the splint or boards will lay flat on the leg. Secure the whole by careful tieing with broad strings or soft cords. The great secret in the successful treatment of simple fractures of the bones of the legs, is the fixing of the leg, so it cannot move until they have united. Feed the horse well, and see every day that the splints on the legs are not loose. Never sling a horse from his feet in cases of fracture, for it will be regretted. If the horse is to be kept in a stall, make a hole in the floor of the stall underneath the foot of the broken leg, so that he can stand with the leg at full length. Bear in mind, no horse will put his foot to the ground till he is able to do so; therefore, leave every thing to the broken-legged, except the matter of securing the broken bones in their place. And by giving him his feed, he may be safely left to himself, and nature will do the rest for him.

\section{Fracture of the Hip or Pelvis.}

Symptoms.-The horse is extremely down in one of 
the hips, and a peculiar loose motion of the hind leg almost giving way every step the horse makes. There are no diseases this can be mistaken for in the hind leg or hip. This fracture is produced in a moment, and even whilst the horse is trotting, he will stop all at once as if he was shot. Fracture of the hip, I say hip, so as the. unprofessional reader will better understand, but it is the bone of the pelvis. The mere name will not amount to much, for the treatment is, let it alone, and it will get well in three months of itself without any interference from medicine or art. Turn the horse out for a few months, either in pasture or in the barn-yard, and give him plenty to eat.

Fracture of the ribs will require no treatment, except it may be a few days' rest. Sometimes a swelling will be seen on the side of a horse having a broken rib, in a few days after the accident. It may form an abscess; if so, have it opened to let out the matter; and if it should not become soft and hold matter, let it alone, as it will do little harm. Or the application for a few times, say once a week for three weeks, of the ointment of iodine. (See Prescriptions and Medicines.)

Fractures of the simple kind, in various parts of the body, take place, and when such is the case, and they do not interfere with any action or function of the body, they can be assisted only in so far as bandage and splint are concerned, so as to secure the ends in proper position. This will require some ingenuity, and cannot be described, as a bone is not always broken at one place, nor is it always of one set kind of break. The means will on these occasions have to meet the ends required of them in the best way possible. Sometimes pieces of broken bone will have to be taken out, where the bone 
has become detached. If this occur in the bones of the leg, of course the horse had better be shot. Where small pieces of bone become loose, it is usually in some of the flat bones, as of the shoulder blade and the lower jaw bone from kicks from other horses.

Fractures of the teeth take place often, and where they are very loose in the head, they had better be taken out altogether, and rasp the sharp points of the broken ones with a file or rasp, to prevent cutting the mouth when the horse is chewing.

Gangrene.-This is a name applied to, or is synonymous with mortification or death of a part characterized by a livid or black color. Gangrene is attended or is ushered in by a sudden giving way of pain, which has after been mistaken for recovery. When gangrene of an outward or external part takes place, a change in the condition of the parts assumes a different aspect, the swelling subside, and touching the parts a crackling sound is produced, owing to the evolution of gas in the parts.

Gastritis Mucosa.-This is a new name to many persons, even well informed in diseases of horses, notwithstanding it is a very common one in the spring of the year, assuming always an epizootic form, and is closely allied to the epizootic catarrh, sometimes called typhoid influenza, (See Influenza.) The chief difference in the symptoms between the two diseases, being the absence of a cough, which is always observed in influenza. The one disease attacking the lining membrane of the windpipe, and in gastritis mucous, the mucous membrane of the stomach and bowels, or in other words, the digestive organs, more than the respiratory. Gastritis Mucosa is 
a fatal disease, if treated by low diet, bleeding, blistering and physicking, because it always assumes a low standard of vitality, or great weakness, Hence the horse so affected, and so treated, has no chance for his life, whatever.

Symptoms.-As before stated, the horse has no cough and the breathing not disturbed. The breath and mouth, is not hot nor dry, but often the mouth is slimy, and to such an extent is this sometimes, it looks as if the horse was salivated. The legs soon swell, or become rounded, or filled as they are often called. The swellings are not inflammatory, nor painful-they contain lymph, or plastic matter from the blood, which disappear as they came, when the strength of the horse gets up again, and the disease subsides. The appetite is entirely suspended, from commencement of the disease. There are one of the many symptoms, which is never absent in this disease, and is very characteristic of its name and seat, and this symptom is the foeces or dung is small, or in pellets, and covered with slime, and portions of the mucous membrane of the stomach and bowels, what the stableman call "very feverish." The great symptoms of this disease is great weakness, and this is the case, almost from the first moment of the attack.

Causes. - The cause of this as well as of all epizootic diseases, are involved in not a little obscurity, and to get out of this state of ignorance, and uncertainty as to the cause, we are graciously pleased to call it Atmospheric. This atmospheric influence, may be either electric, a poison, or chemical element, capable of altering or changing the various parts or portions of the body, most exposed to its subtle influences. However, this disease as before stated is a disease of the spring of the year, 
and commencing as the hermetically sealed earth begins to open its pores, to the rain and sunshine of spring. May there not be deleterious emanations from the earth, or at least after great frost or snow, is there not during the process of thawing a colder air or gas given forth from the thawing process, than the animal is breathing, a few feet higher up from the ground? This is one of the points entering into the celebrated controversy between myself, and the distinguished Professor, R. E. Rogers, of the University of Pennsylvania, on the salt and slush question, a few years ago, whether it was, or was not injurious to the health of man or horse, to sprinkle salt upon the track, to thaw the snow from them. If my argument did not prevent further use of the salt upon the track, it did one good, by making it obligatory for the railway companies, to keep all gutters and inlets clear, to allow the slush to escape at once from the street. So I think it is clearly established, that the cold emanation from the soil, during the process of thawing or breaking up of winter, are great and exciting causes, not only of this disease, but of many others in both man and beast, and which have hitherto been called atmospheric. And in connection with this condition, we have in horses at least, a want of their usual protection, for with the warm sun of spring, the animal throws of his hairy coat, thus as it were unnecessarily exposing himself to these insidious causes of disease.

Treatment.-When cases of this and other diseases of the same type (gastritis mucosa) first came under my care, I treated upon different principles and with different medicines than what is here recommended; I look back with extreme dissatisfaction on the false doctrines and false teachings of the book and the schools which have led 
many inquiring minds astray since their day of teaching commenced. The treatment now recommended is sound, scientific and successful, and in a very short time the horse will be at work again, as if nothing had been amiss. The first day of the disease, give, every 4 hours, 20 drops of the tincture of aconite root in a little cold water; next day, give the tincture of nux vomica in fifteen drops every four hours in the same way, till the horse is well, which usually will be about the sixth or seventh day, and sometimes even sooner. If, however, the case does not improve, and the appetite not good, give powdered carbonate of ammonia and gentian root, each three drachms, to a dose morning, noon and night, in addition to the tincture of nux vomica. These medicines will have to be mixed with cold water, and drench the horse out of a strong-necked bottle or ox's horn cut slanting at the mouth. Keep the ammonia in a bottle tightly corked till it is used, as it loses its strength by exposure to the air. Let the horse have as much cold water to drink as he wants; and for this purpose a bucketfull should be kept before him. Pure air and good ventilation should be insured to all sick horses. Green, or soft feed should be given from the first day if the horse will eat it. Green feed all the time of sickness will be of advantage, but oats will have to be given in addition, to support the strength and vital powers of the system, to enable them to throw off the effects of the disease. Such, then, is the manner of curing a disease which has destroyed many horses, even when treated by men calling themselves veterinary surgeons, or at least horse doctors. (See Influenza and Rheumatism.)

\section{Glanders.}

Glanders.-The following synopsis of a lecture deli- 
vered by myself and.published in the Evening Bulletin of December 21st, 1865, will answer for the history and nature of the disease called glanders. The treatment will be made more plain for non-professional readers.

Dr. Robert McClure, Veterinary surgeon, delivered a lecture on Tuesday evening at the Veterinary College. His subject was "Glanders in Horses." He said: Glanders is a disease dating from the time of Xenophon, or four hundred years before Christ, and we are assured by Hippocrates, (ruler of the horse) that confirmed glanders was incurable, and that it was then known by the name Profluvium Atticum. Veterinary surgeons recognize two varieties of Equinia in the horse, viz: Equinia mitis. Contracted from horses with greasy heels (Paronychia Equi and Equinia Glandulosa a dangerous disease, and readily communicated to man. Glanders is unknown at the tropics and at the poles, and is not seen where struma is not a disease of the people. It is a domestic disease. The assignable causes are many; among which may be enumerated starvation, filth and debilitating diseases, as strangles, catarrh and lung-fever, or, indeed, any disease capable of generating pus; and this pus being absorbed into the general circulation, thus forming a ferment, a Zumin, or a leaven, as the Bible has it, within the blood, and the effort of nature, to get rid of this offending matter, is seen in the ulcerations of the lining membranes of the nose. The recent experiments of Professor Giovanni Polli, of Milan, seems to corroborate this view, as he has produced glanders and other Zymotic diseases in seventy dogs, by injecting into their blood in some cases fetid bullock's blood, pus, and glandered products, and neutralizing the ferment so set up by the administration of an alkaline sulphite-a new intero chemical doctrine-on the 
the same principle of arresting the vinous fermentation set up in a vessel of cider by adding to it a preparation of lime. The experiments of this distinguished professor, enable the veterinary surgeon to extend his usefulness, and the domestic physician to snatch many a useful life from an early grave. How many brave.men have stood the storm of battle in the late war-adimitted to the hospital with perhaps a shattered bone-amputation is performed, the case does well for a few days. The kindhearted surgeon sees a change for the worse:-appetite gone, the rigor and chill supervene till it is too plainly seen that the pus from the stump has been absorbed into the blood of the unfortunate man; fermentation is set up, and death staring him in the face, and no power to save. The discovery of a ferment and its antidote have changed the scene from death to that of life. So, likewise, with the veterinary surgeon, however ignorant and slow some of them are to see and understand, it will be enabled to preserve the life of many a useful animal to its owner, thus adding very materially to the happiness and prosperity of this great and prosperous people.

Glanders are recognized by ulceration of the lining membrane of the nose, or the formation of pustules, and commonly situated in the septum nasi; these pustules soon ulcerate and discharge pus of a greenish color, rapidly drying up when spread over the nostrils, and sinking in water, owing, as is supposed, to it containing no oil or pus cells, but principally albumen. There is one phenomena never absent in this disease, and that is the enlarged gland under the jaw; hence, the common name of the disease-glanders. There are, said the Lecturer, many wrong ideas entertained, not only in regard to the contagious nature of the disease, but also in regard to its 
incurability and even fatality. Glanders is no more contagious than the heavy, stinking discharge from the nose of some horses with catarrh; as the pus of an abscess on the nose of a horse with a cold, when introduced into the blood of healthy animals will produce a ferment, which explains the reason why horses with a cold terminates in glanders, because of the absorption of the pus. This will be readily understood, when it is said the horse is running or bordering on glanders. Horses affected with chronic glanders will live and work for years, which fact, being well known, has caused dissatisfaction with local laws, prohibiting the use of glandered horses.

Fresh specimens of sections of the lungs, nose, and other portions taken from a glandered animal, were placed at the disposal of the Lecturer for the purpose of illustrating to the audience, showing the morbid changes effected by the disease. At the close of the lecture, the doctor showed the manner of generating nascent hydrogen for the purpose of detecting the presence of the alkaline sulphite in the secretions of horses under its effects for the purpose of the cure of glanders. Before touching upon the plan of treatment, the reader is referred to the article Farcy.

Treatment.-The proposition of restoration in a disease of this kind, is the destruction of the ferment, the remoral of its products or effects, and the improvement of the bad habit of the body by enriching the blood.

To remove or neutralize the ferment or poison, give one-half to one ounce doses of the sulphite of soda at night, in cut feed for several weeks, and five grains of the powdered Spanish fly along with it, which will act not only as a powerful tonic, but as an agent whereby the product of the disease will be removed from the body 
of the animal by the kidneys. This treatment will not interfere with the other medicine, which is powdered gentian root, three drachms; powdered sulphate of copper, two drachms. Mix these articles, and give the whole for a dose, and give one dose morning and midday.

These medicines will have to be continued for a long time, not only to cure the disease, but to improve the health. The horse must be well and highly fed, and removed from other horses while the treatment is going on. Change the feed often, so as to get all the elements that the blood requires, and to keep up the appetite, for if the appetite fails, no cure can be made.

Remember.-Glanders associated with tubercles of the lungs, cannot be cured, and it then partakes rather of the nature of consumption than of simple glanders without tubercles, which is easily cured.

Glass Eye.-(See Eye Diseases.)

Gleet.-(Nasal.) - This term is used to denote a thin, transparent discharge from the nose in cases of coryza, and as a sequel to catarrh and cold in old and debilitated horses. Whilst there are no ulcers on the lining membrane of the nose, or no enlargement of gland under the jaw, the case may be dismissed as simple gleet, which can be readily cured by good feeding and a few tonic powders, such as powdered sulphate of copper, three ounces; powdered gentian root, four ounces; powdered Spanish fly, one drachm. Mix, and divide into twelve powders, and give one powder once in the twenty-four hours. These powders will last two weeks, and can be renewed if necessary. Give the powders in large bulk or cut feed, so as to protect the coat of the stomach from the effects of the Spanish fly. 
Granulation.-This means the little red portions of flesh, which grow in and fill up holes made by wounds. Sometimes these grow too fast; then they are unhealthy, being soft, and grow beyond the edges of the wound. To prevent this, sprinkle a little powdered bluestone, or a little sulphate of zinc, and the wound will soon heal level with the surrounding surface.

Gravel in the Foot. - This name conveys an impression that sand or gravel has got into the foot, which is often the case from cracks or other openings in the foot, whether from above or below. Wash out the sand, if possible; if not, remove some of the horn, and wash out well, and fill up the hole by shoemaker's wax applied hot, and smoothed over by the hand previously wet, so the warm wax will not stick to it. If the opening be in the sole, shoe with leather soles, tar and cotton, until the hole has grown out or closed up.

(1.) Grease.-This is a disease of the heels and legs of horses, characterized by an unsightly condition of these parts. The whole being the result of suppurative inflammatory action of the skin and heels of the hind legs, usually, but sometimes of the fones, is more common in coarse-bred western horses, and of heavy breeds, than in well or fine bred horses.

Cause.-Sudden changes of the temperature of the earth, whether from heat to cold, or from wet to dry. This disease always follow sloppy or wet streets, stables or lands, producing a relaxed condition of the parts from too much moisture.

Treatment.-Keep the legs clean and dry, and aprly a mixture to the heels twice in the twenty four hours. Water, one pint; Sulphuric acid, two drachms; Corro- 
sive chloride of mercury, one drachm. Mix, and shake up before using. Many cases are readily cured, by simply keeping the heels clean, and anointing with glycerine, or lard, having no salt in it. It must be confessed by every body, who has had any experience at all in the treatment of this disease,-that it is very uncertain of an early cure; some cases will be cured in a short time, and in others it would seem that the discharge would never dry up and be healed. For cases that prove obstinate, the following plan will effect a cure, when other vaunted remedies have failed: Take one box of concentrated lye, and dissolve it in two quarts of water, and bottle up for use when wanted, in the following way: Pour a wine-glass full of the solution of lye, into a small half bucket of cold water, and wash and bathe the heels and legs for half an hour, morning and night. A great change for the better will be seen in a day or two. This wash seems to have the power of relaxing and softening the skin, and at the same time causes the legs to sweat greatly. Dry them as often after the bathing as you like, there will pour out great quantities of moisture from the skin as soon as you have done.

(2.) Grease Shot of.-This is a different disease from the one bescribed, from the fact that it attacks only one leg, and that one of the hind ones, and comes on in a night, without any preceeding symptoms whatever, and hence it is called a shot of grease. There is no cracking of the skin of the heels or legs, but it remains whole and unbroken.

Cause-Robust stamina, or too fat and full of flesh, and to get rid of this superfluity, plastic lymph is thrown into one of the hind legs, which causes swelling of the leg to an enormous size. If this material were thrown from 
the blood into one of the fore legs, where the nearness to the heart increases the activity of the capillary circulation, matter would not remain as it does in the hind legs, which are so far from the centre of circulation. This disease is not unlike the phlegmassia dolens or as milk leg in the human family.

Treatment.-If the disease be observed early or before the leg becomes hard, take about one quart of blood from the neck, and give slop feed, that is, bran with plenty of water in it. Also, give one ounce doses of the sulphite of soda once in the day for a few days, and bathe the legs three times in the day with the same solution of concentrated lye, as is recommended in grease, (which see.) If the swelling does not lesson in two days after these various agents have been employed, then incisions of an inch in length through the skin will have to be made for the purpose of letting out the imprisoned fluid before the arteries of the legs have become plugged or filled up, which constitute the thick or fat leg so often seen in horses in large cities. In addition, the leg will have to be bandaged pretty tightly with a broad bandage, and the leg to be still bathed three times in the day with the solution of concentrated lye. Many good horses have been rendered of little value from want of a knowledge of this disease and its proper treatment.

Gripes.-This name is sometimes applied to colic, (which see.)

Grogginess.-A term meant to convey the idea that the horse does not travel very steady in front, and from contracted or bent legs at the knee, or from soreness in the feet from a previous attack of founder in the feet, (which see.) 
Grunter.-This name is used or applied to horses which give forth a grunting noise. One condition giving rise to wheezing, roaring, whistling, piping, and rattling, will, with slight modification, produce a grunter.

Observe-If the collar is not too tight on the neck, interfering with the free passage of air in and out of the windpipe.

Causes,-Generally from some thickening of the glands of the neck or of the windpipe from the effects of bronchitis or distemper, not treated, or improperly treated, by not supporting the strength, whereby all thickenings are taken up, or reduced and even prevented.

Gullet, Obstructions in.(See Choaking.)

Gun Shot.-This is, excepting in times of war, a rare occurrence, and the treatment consists in extracting the bullet, and healing the wound as for an ordinary sore. The bullet is extracted by an instrument represented in the accompanying cut, and is called Bullet Forceps. The instrument is represented as grasping the bullet; also in the way it is introduced to extract the ball.

Gutta Serena.-(See Eye Diseases.)

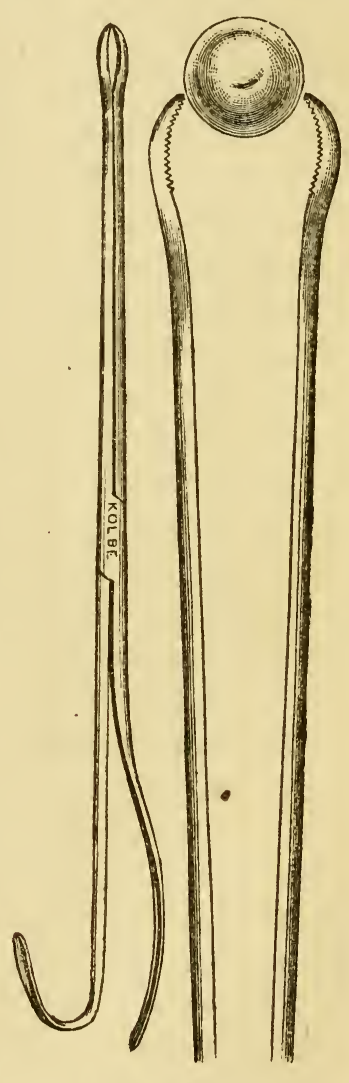

BULLET FORCEPS. 
Diseases of the Heart.-Of diseases of the heart, little need be said; and as little can be done in the way of cure, so all I propose under this head is merely to name a few of the altered conditions of the heart, which are all embraced in the sweeping term "Heart disease."

(1.) Enlargement of the heart.

(2.) Wasting or shrinking of the heart.

(3.) Foreign bodies in the heart.

(4.) Fatty degeneration of the heart.

(5.) Inflammation of the heart.

(6.) Ossification of the heart.

Such, then, are some of the distinctions made, when speaking of diseases of the heart. And as an illustration of what a horse with heart disease can do, and what can, and is sometimes done with such an animal so affected, I reproduce an article from the Scottish Farmer, which may be of interest to some of our American farmers.

"If one of the busy paternity who delight in a deal, on a dark Wednesday night, in the grass market, were asked what sort of horse he wished to procure, as a safe investment for his ill-gathered coin, he would certainly prefer a good-looking Clydesdale, sound in limb; not a roarer, but with a mysterious screw loose, which few, including the horse-Coper himself, can discover. Such an animal has been turned over many and many a time within the last three weeks, in or near Edinburgh.

Our readers will remember a second article on heart disease, in which three cases were specially mentioned, and one amongst them, under "observation." A fullsized, active gray mare, apparently fit for a goods van, having been treated for a cold by a blacksmith, proved to be 
suffering from dilation of the right side of the head. She was looked upon with interest by a strong muster of students, who hoped to see further into matters, and proposed to buy the mare, to be destroyed, as the only humane method to treating her. But, as we are informed, a friend, seeing the animal with swelled legs, only thought he could cure the case, and urged that she should be sent to his farm, where he would put her to the plough. In opposition to professional advice, this was agreed upon, and the students disappointed of their legitimate prize. Not many days elapsed before the farmer found that he had undertaken a hard task, in attempting to cure the swelled legs. Seven dollars and a half were realized for this likely-looking animal, and we believe a smith, near Edinburgh, was the purchaser. Here, the trickery commenced; and a simple countryman greedily closed a bargain, which enriched the smith by forty-two dollars and fifty cents. The animal's wind, was, however, wrong and cartwork would not suit her; so that, in despair, an exchange was effected with some of the ingenious dealers in horseflesh, in this town. Exchange followed exchange, and it proved very troublesome to trace the animal, when at last, in broad day-light, she falls, by the auctioneer's hammer, to the lot of a man from Glasgow-side.

Many a twenty dollar note can be made out of a poor animal thus knocked from hand to hand, until, in the course of nature, it drops dead in harness. The history of the case is as interresting as would have been the post mortem to the students; and it will be found that one of the most remarkable features in such cases, is the length of time that animals retain a selling appearance, though absolutely unfit for any exertion."

Heaves.-This is a term in frequent use, but not so 
well understood as it ought to be, seeing its importance as to the proper value of an animal. Heaves, then, may be defined a difficulty in breathing, whereby, the value and usefulness of the horse is seriously impaired. There is every degree of intensity to be seen in this disease. Some animals are so seriously affected, that it is hard to look at the distorted efforts made in the art of respiration. The deep and not suffered-to-be-completed respiration, tells the tale of great asthmatic effort and oppression. In others, it is so slight that only experts can observe it; not from the quickness of breathing, nor yet of its depth; but of a peculiar double beat or hitch, differing from all other varieties of breathing, either of fever, inflammation, or debility, it is still single and separate.

Causes.-Debility of the parvagum nerve; for if this nerve is derided or cut in its coarse, heaves, or broken wind, is set up at once. What the real condition of this nerve is, which gives rise to heaves, is not at present known; and it is difficult, in many cases, to trace and distinguish any alteration of the nerves, as they may retain their color, yet their influence may be greatly impaired. For two reasons I have said debility. First, because, if it was cut, or had entirely lost its influence, the animal would die in forty-eight hours. Second, because, by the administration of powerful tonics, the symptoms of the disease are greatly relieved, and in some cases they entirely disappear.

Treatment.-Horse-dealers, in order to allay the symptoms, that is, the peculiar breathing, give an ounce each, of powdered sulphate of iron, gentian and ginger root. It is from the large dose that is given, and repeated for a few days, that gives or imparts a tonicity to the nerve. Although I have here spoken of this matter, I do not 
wish to be understood as advocating such a business, neither in the attempt to cheat nor deceive any one; but the large dose of iron meets my decided disapproval, as being highly injurious to the horse, although the giving of round or spherical shot, as is practiced in England, is much worse. The treatment, whatever that may be, to do good permanently, must be by a gradual and progressive improvement; five grain doses of arsenic, given once in the twenty-four hours for two weeks; then, after a week's intermission, commenceing as before, will cure many cases Feed the animal with feed in small. bulk. Give as little hay, or rough feed, in large bulk, as possible. Improve the condition of the horse by every way or means, and you will relieve the animal.

Heat.-General heat of the skin indicates fever;-local heat, inflammation.

\section{Hepatic Diseases.-(See Liver Disease.)}

Hernia.-This is a name given to ruptures, (See ruptures.)

Herpes.-A named used in skin diseases, (See mange and skin diseases.)

Hereditary Diseases.-No one, of any observation, can deny that hereditary influence exists in the production of disease. This influence, it must not, in the production of disease, be considered in the light of too many, that if a horse or a mare had disease, their young ones will have the same disease, also, - not at all. It is by whatever change or alteration of structure, action or function, that existed in either of the parents, that was the cause of disease in them, and these same forms which existed in them, are likely to be transmitted to the offspring, thus carrying the various formations of struc- 
ture, which will ultimately end in producing diseases of like character. A great number of those affections, which are usually styled hereditary,-do not make their appearance until years after their birth, because it requires time and work to develop them. Few persons would expect a horse with cow hock, (which see), to become curbed without work, as a secondary cause. There is one other point worthy of remark, when speaking of hereditary disease, and that is many animals, which have been bred poor, have been animals badly fed and cared for, whereas if good feeding and care, had been bestowed upon them, it would have gone a long way in lessening the certainty of developing hereditary diseases in themselves, and their offspring. This is every day being illustrated in the family of man. There are several rules laid down to be observed as measures to prevent and modify these conditions, which result in producing disease of hereditary predisposition. And there have been receipts found in the temple of Æsculapius, and said to be in the handwriting of Hippocrates himself, for the purpose of mitigating the hereditary conditions, which are so often seen in the human family. Although these rules cannot in all cases be applied to animals, nevertheless, much can be done; but the better way will be to avoid breeding from diseased animals. So long as like begets like, so long will we have hereditary disease among horses.

Hide Bound.-This, properly speaking, is not a disease, but the symptom of bad condition, "out of sorts," debility, etc.

Treatment.-Feed in large bulk, or mixed feed, cut hay, corn meal, and bran in good proportion, with no more water than will keep the particles togther. Give 
the following powder every night in feed for twelve nights:-Powdered sulphate of iron, three drachms; powdered gentian root, four drachms. Mix. If the animal is fat and yet hide bound, substitute the following, instead of that above recommended:-Take sulphuret of antimony, three drachms; sulphur in flour, three drachms; sulphite of soda, half an ounce. Mix, and give in one dose, and give one dose every night for two weeks. If in the winter, put on an extra blanket. If in summer, give cut grass to eat.

Hip joint Disease.-Happily, this is a very rare disease in horses, and does not make its appearance so soon as in man, and is always the result of accident or injury, and is a joint affection, not of the muscles, because the mass of muscles are so very great over the hip, that it is not an easy matter to sprain them. I speak of this here, because among horsemen it is a great bug-bear, and often occurring in their ideas: whereas, in ninety-nine cases out of a hundred of their so-called hip and whirl bone disease, the lameness will be found in the hock joint. Why do they jump at the conclusion that the lameness is in the hip? Because in every movement of the hock joint, the vibration or hitch is more distinctly seen by the altered action of the muscles of the hip, as when the hock joint moves but partially, and not completely, it is not so readily seen at the hock, but at the hip. Hence, this is but the old adage, cause and effect, but in this case the horseman has them reversed. (See Spavin.)

Symptoms.-Lowness or falling in of the hip; a peculiar manner of moving not indicating inability as is seen in fracture of the pelvis bone, but of great pain and diff- 
culty, not yielding or bending low down on that quarter when the horse takes a step. The leg appears shorter, and is placed when standing slightly under the body, and not doubled up, or standing upon the toe as is seen in cases of hock joint disease.

Treatment.-Absolute and entire rest for a few weeks. The application of cold water cloths over the hip, taking them off at night, and applying them in the morning again. This will have to be kept up for a week at least, so that all heat and tenderness will be removed before any irritant can be used to the parts. Never apply hot liniments to a part already too hot and painful. Then apply by rubbing with the hand over the parts every second day the following:-Oil of turpentine, one ounce; oil of olives, two ounces; creosote, one ounce; mix. This will not only act as an irritant, but as a powerful sedative to the nerves of the parts, causing relief from pain, and consequently the animal can be made useful.

Hock, The.-This is an important joint or part of the horse, and is the seat of many diseases, causing lameness. In the majority of hind leg lamenesses, the hock is the true situation. This fact is not apparent to the non-professional person, as the hock while diseased does not swell so often as other portions of the body, or legs when diseased. Bog or blood spavin and thorough-pin, a blind man can almost see. With these exceptions I say, hock joint lameness in the majority of cases is charged to the hip or somewhere else, simply because persons fail to see any peculiarity, even when pointed out to them. (See Stringhalt.)

Sprain of the hock joint is to be treated with cold water cloths for a few days, and the application of the 
following, once every second day, for a week, with friction or rubbing:-Oil of turpentine; oil of olives; equal parts mix. This is a simple, cheap, and good liniment, and will answer every good purpose. For diseases of the hock joint, see Spavin.

Hooks in the Eyes.-This is a term in use by country folks when referring to the peculiar action of the membrana nictatans in cases of locked-jaw, (which see.) Many persons have advised that these useful membranes should be cut out. Nothing could be further wrong than this; for it is merely the effect of a cause. Rather remove the cause, or cure the locked-jaw, and the protruding of the hook or horns will cease. This is the only true plan to adopt.

Horse, Natural History of the.--The horse comes under the division vertebrata, class mammalia, tribe Ungulata, order Pachydermata, family Solipeda.

Horse Fly.-This comprehends the gad or breeze fly, Gasterophilus oestrus. The spotted horse fly, $G$.

- Equi. The red-tailed horse fly, $G$. hemorrhoidalis. This last fly deposits her eggs on the lips of the horse, and the former glues them to the hair of the legs. These various eggs are ultimately taken into the stomach, and in one year they have become sufficiently matured that they are thrown out to the outer world to get wings, and finally fly about and propagate their kind in the same manner as the parent stock. (See Worms.)

Hydrothorax.-This is a name given to water when it accumulates in the chest. Hence, hydro, water, and thorax, the chest.

Causes.-Debility from the effects of inflammation of some of the organs within the chest. 
Treatment.-Tonies to improve the general health, and medieines to draw off the water by the kidneys and bowels. An operation is recommended by which the fluids are drawn from the side by means of a trochar. (See Dropsy.)

Hydrocele. - A collection of fluid in the scrotum of stallions.

Ireatment.-Paint the scrotum with the tincture of benzoin or iodine. If these remedies are not successful in causing absorption of the water, the skin of the scrotum will have to be opened to let out the fluid.

Hydronemia.-A name used when speaking of the blood, when it contains too much water-water in the blool. (See Dropsy.)

Hydrophobia.-This disease, happily, is rare in a practice of thirteen years, and doing much in the treatment of diseases of horses, and being well acquainted with the practice of other persons, I can only record three cases, and one of these was a mule. This disease is sometimes called water dread and canine rabies from the fact that it is only generated in the $\log$ and feline species of animals.

Cause in Horses. - Bites of the mad dog, and sometimes the bite from the common cat is capable of producing the disease.

Symptoms.-In those cases that came under my notice, the symptoms were anything but like each other. In one animal, the propensity to bite at objects was more severe, and in another, the horse would walk and look about him, utter a peculiar sound, and lie down and get hold of the heels, and part of the arm of the fore leg with his teeth till he had them bleeding, get up again, and 
walk about without any apparent object in view. But the destructive impulse attributed by some authors, I think, is merely the fancy of an inexperienced mind in such matters. The symptoms of the mule differed from each of the horses, inasmuch as he would at times eat hay very ravenously, stop all at once, and with the peculiar sound not like the bark of a dog, but of such a character which cannot be described, and lie down and have a good roll to himself; then he would run at any person within his reach with open mouth. But in no instance would any of the cases attempt to bite at anything not endowed with animal life. All of these animals were confined in enclosures trom which they could not eseape. Any person having the least experience with horses, will observe a peculiarity at times, which will appear not to belong to common discases. These animals lived about thirty-six hours after the active symptoms of the disease set in. (See Bites of Mad Dog.)

Hypertrophy.-This name means a state of certain organs increased in size, and decreased in power. The heart, kidneys, spleen, liver, and other parts of the body, are liable to this disease.

Hysteria.-This is a disease which is sometimes seen in mares only.

Causes.-Irritation of the uterus, or of some of its nerves.

Symptoms. - Great exeitement and incapability of standing, and it appears as if some of the bones of the back or loins were broken.

Treatment.-Give twenty drops of the tincture of aconite root every four hours, whilst the symptom lasts. Build up the strength of the mare by the following:- 
Sulphate of iron, three drachms; gentian root, three drachms. Mix, and give in one dose every day for a week or ten days. Give good feeding.

Caution.-Unsafe to use-will return again.

Indigestion.-However much, man in the sedentary walks of life, may be the subject of this disease, the horse with a task-master is comparatively free from it. Cases do occur occasionally in our large cities, where in too many cases the horse is left standing in the stable, twenty out of the twenty-four hours. Idleness begets indigestion, and indigestion begets crib-biting, or windsucking, and between them the poor horse looses flesh, condition and spirit. (See crib-biting.)

Treatment.-Send the horse to pasture, and when he returns give him regular feed, and regular work to prevent a return of it.

\section{Infection.-(See contagion.)}

Inflammation.--Inflammation of the various portions or parts of the body will be found treated of under the name of the organ or part affected.

Influenza.-This is a name which is properly applied to an epizootic catarrh of frequent occurrence in the spring of the year. Indeed it is very rare that we see a cold run its course as such, without some complication of one kind or another.

Symptoms.-A chill or shivering fit, succeeded by increased heat of the body, with fever and irritation. Loss of appetite, cough, discharge of mucus from the nose, watering of the eyes, great prostration of strength, followed in a day or two with swellings of the legs, and in bad cases, of the belly, breast, and in males, of the 
sheath; Such is a true and succint account of the symptoms of this disease. The symptoms will vary as in other diseases, with the intensity of the affection.

Causes.-A subtle poison in the air, sudden changes in the dryness or moisture of the earth's surface, easterly winds, cold accompanied with dampness in the air, these are conditions which too often accompany or precede influenza. Influenza differs from an attack of common cold, chiefly in the severity of its effect, causing more fever and greater debility. In England it was first observed in 1819, and again in 1832, and more or less ever since. In the United States it first manifested itself in 1856, and is still seen every spring and fall with symptoms more or less severe.

Treatment.-The mortality in this disease is great, when treated according to the books, which our publishers issue from this press, old English books, with new dresses cut to the fashion. Bear this in mind, when undertaking the treatment of a disease of this kind, that one step wrongly taken, can never be recalled. Place the horse in a cool (not cold) and airy place, and put a light covering upon him, and give twenty drops of the tincture of aconite root in a little cold water, every four hours, till five doses are given. Place plenty of cold water before the horse so that he can drink as much as he wants. When the aconite has been all given, commence with fifteen drop doses of the tincture of nux vomica, also, every four hours, and continuing it for a few days, and if the animal improves and the appetite return, nothing more in the way of medicine need be given. Recovery being slow, and the appetite poor, give the following powders, morning, noon and night: Powdered Carbonate of Ammonia, three ounces; Pow- 
dered Gentian Root, two ounces; Powdered Pimenta Berries, two ounces; Mix, and divide into 12 powders, and give them mixed in a little cold water, and drench the horse out of a strong mouthed bottle. The powders will have to be wrapped well, so as to keep them from the air, so as not to loose their strength. Twenty drops of commercial sulphuric acid may be given occasionally,

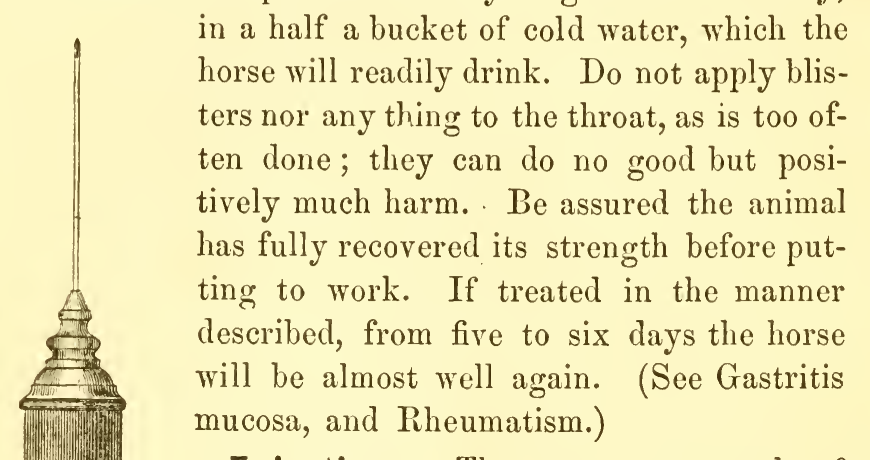

Injections.-These are composed of warm water, soap, and a handful of tablesalt. The water about luke-warm. The usual way to give injections, is by means of a large syringe, capable of holding a quart of the fluid. The diseases which call for injections, are the various varieties of cholic. Few medicines will cure colic without the aid of injections; whereas, colic, in very many cases, can be readily cured by the injection alone. Therefore, never put confidence in any person who undertakes to cure colic, without injections of warm water, soap Injection Syringe. and salt. 
Instruments. - The various cuts of instruments, represented throughout the book, explain themselves. The few here placed, may be explained as follows:

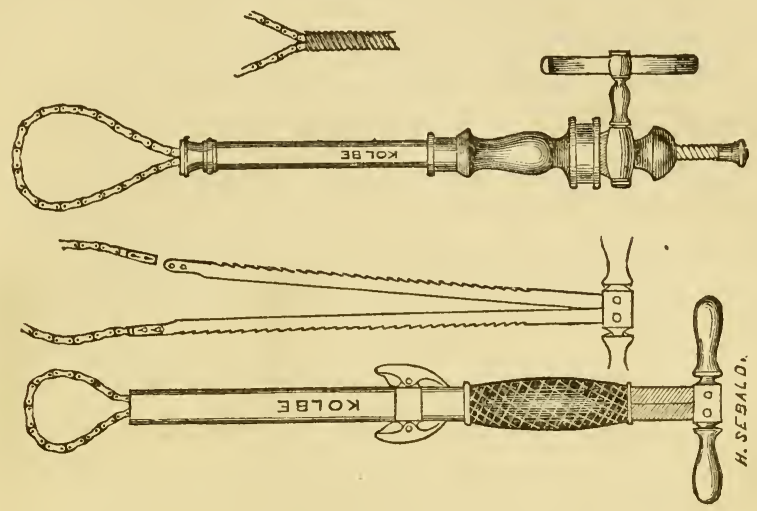

No. 1.

No. 1, is two patterns of the ecraseur, as manufactured by D. W. Kolbe, surgical instrument maker to the University of Pennsylvania, 15 South 9th street, Philadelphia.

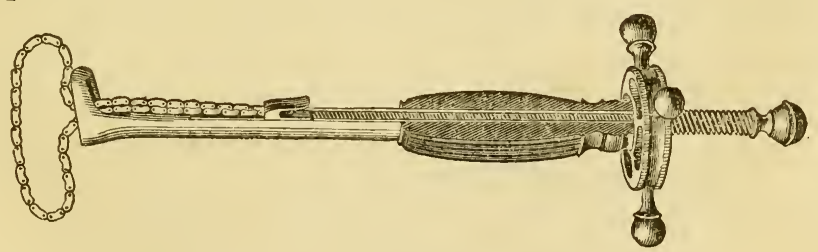

No. 2.

No. 2, is a representation of the same instrument, as manufactured by John F. Kumerle, 111 South 8th street, Philadelphia. The ecraseur is now extensively and successfully used for castrating colts, and for the removal of tumours. The object of using this instrument, is, that no bleeding follows its use.-(See Castration.) 
DISEASES OF THE HORSE.

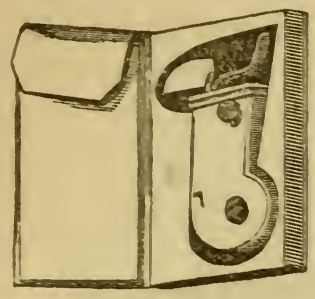

พัo. 3.

No. 3 , is a spring lancet, and was inrented by a Mr. Weiss, of London, for the use of farmers who could not use its more surgical prototype, the lancet. This Tas at a time when bleeding was thought to be the great panacea for all the ills and aches, to which

man and beast is heir.

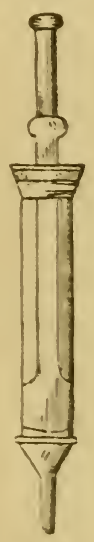

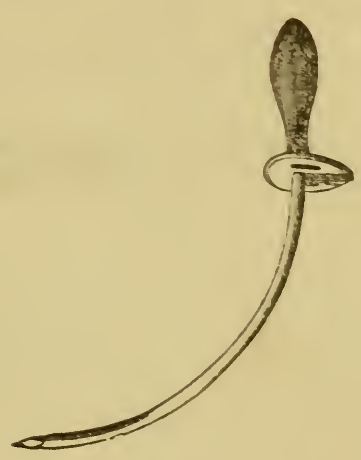

No. 5 .

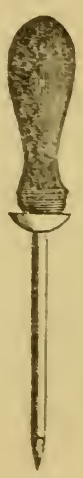

No. 6.

No. 4.

No. 4 , is a small stringe, used for injecting medicines into the carities of tumours, to destroy their diseased walls.

No. 5, is a curred trocar and canula, and is used for piercing dropsical strellings and tumours, to run off the fluid. The trocar is drawn out after the parts have been pierced, and the canula remains as a tube, whereby the mater escapes.

No. 6, is a straight trocar with canula, and is used in different sizes, and for various purposes. Its first, and 
most important use, is for piercing the rumen of cows and oxen, when attacked with hoven. Second, it is used occasionally for piercing the horse, in cases of flatulent colic, and in drawing off the fluid or serum, accumulated in the chest from disease of the lungs and pleura. 'Third, a small size of this instrument, is used in piercing the head of sheep, affected with hydatids. After the trocar has entered the hydatid sack, the trocar is pulled out, the canula remains, the point of a small, and well-packed syringe is placed in the head of the canula, and the suction-force draws the fluid into the syringe, and the small cysts into the canula; so it can be caught, and pulled out by means of a pair of forceps. A trocar and canula should be kept by every farmer who has a cow or an ox.

Intestines.-Introsusception, or an entangling of the intestines, sometimes takes place in horses, and proves fatal.

Causes.-Bowels empty, and the horse being driven fast.

Symptom.-When the horse comes in, he is observed to be uneasy:-lying down, pawing with his feet, following closely the symptoms of colic. The difficulty, or impossibility of procuring relief, is only seen on examination, after the horse is dead. (Sce Bowel Diseases.)

Itch.-(See Mange and Skin Discases.)

Interfering.-This name is in use, when speaking of a horse hitting himself on the inside of the pastern joint, either on the hind or fore leg. Sometimes it is called cutting. It is usually done with the side of the opposite foot.

Causes. -The blacksmith is many times wrongfully blamed for shoeing the horse, bcause he interferes. There 
are cases, no doubt, where a little observation and care, on the part of the shoer, would have prevented it. From much observation, I am satisfied that the chief cause lies in the weakness of the horse, particularly in the spring of the year. Horsemen well know that their horses did not interfere in the winter months, when the weather was not oppressive, and the horse in excellent spirits; and no changes have been made in the man or manner of shoeing.

Treatment.-Give a few powders of iron and gentian in the feed, to restore the horse to strength. (See Medicines.)

Jack.-A small point on the inside of the hock-joint of the horse, affected with bone spavin.

Jaundice.-This signifies tbile in the blood:-biliary intoxication, tinging the membranes of the nose, mouth, etc., with a yellow color. (See Liver Diseases.)

Joint Diseases.-The diseases of the various joints in the horse, are many. Among them may be enumerated four varieties of spavin, bone, blood, bog and occult, all of the hock joint. Of the patella, in the form of dislocation. Of the hip, or whirl-bone joint, ulceration and sprain. Of the joints of the back-bones, caries and ulceration. Of the foot, coffin-joint, commonly called navicular-joint, lameness. Of the pastern joints, anchylosis or stiff-joint. Of the lower pastern, ring-bone. Of the knee-joint, stiffness and open joint. Of the point of the shoulders, ulceration and bulging out of the capsular ligament of the joint. Wind galls, of almost all the joints, more specially in the pasterns. The cause and treatment of these affections, will be found under their proper heads, throughout the book.

Jugular Vein.-Inflammation of. This may be merely 
a simple swelling, after bleeding, caused by bruising the parts, by too great force applied when bleeding, or by closing the wound too tightly, causing extravasation of blood, between the skin and the fascia. Inflammation of the jugular, may be of great magnitude, involving that vessel the whole length of the neck, above and below the wound made by bleeding, ultimately causing its entire obliteration. And, occasionally, the inflammation extends to the brain itself, destroying life. Happily, bleeding is not now recommended in the treatment of disease; consequently, this affection will be among the diseases of the past.

Treatment.-Remove the pin, or whatever has been used to close the wound, and apply a piece of blue-stone to the bleeding sore: this may be used once a day, for a day or two. Hot fomentations, or a small poultice, should be applied to the part, to abate the inflammation or irritation. The fomentation will have to be applied, the whole length of the thick corded vein, to cause its relaxation. Cut the feed for the horse, so as to save the movements of the jaws, thus giving rest, as much as possible, to the parts affected.

Kidneys, Diseases of.-The most important of which is that already described under the head of diabetes, (which see.) Hæmaturia or bloody urine, is occasionally seen in horses, more often in cattle, and consists in a diseased state of the kidneys, from violent strains or accidents.

Caleuli, or stone in the kidneys, are often found in the kidneys of horses, and cause considerable irregularity in making water. 
Treatment.-Occasionally give thirty to forty drops of muriatic acid, in a bucket of cold water to drink.

Bloody urine may be treated by warm-water cloths, laid over the back or situation above the kidneys, and give flax-seed tea to drink, to soothe the parts. Give no saltpetre or other medicine.

In old horses, as is in old men, considerable chronic disease of the kidneys exists. Although not much in the way of cure by medicines can be done, a great deal can be done to soothe the parts, by soft and soothing feed and drink, such as steamed or boiled feed, and flaxseed tea, cut grass, and other green feed, with plenty of cold water at all times to drink. The more fluids that go into the body, the less irritation of the bladder and kidneys. (See Bladder Diseases.)

\section{Knees Broken.-(See Broken Knees.)}

Knee Joint-Loose Cartilages in.-Small loose cartilage is sometimes found floating in the knee joint of horses, as well as of man, and is the cause of much of the obscure lameness that is so often unaccounted for, and so far as I am aware have never been noticed by any writer. The lameness is very sudden and passes off as if nothing had happened, constantly coming and going.

Treatment.-Removal of the body from the joint by first getting it into a corner, and holding it there, and cutting and taking it out, this is too dangerous an operation for every person to undertake, as it is cutting into a synovial cavity.

Knuckling.-This is a peculiar loose double action of the pastern of the hind legs, and is a symptom of rather than a disease of itself. 
Cause.-Disease in the hock joint, and accompanies occult and bone spavin, weakness in the part from the effects of sprains, or other injury.

Observe.-Knuckling of the hind pasterns, is a symptom strongly indicative that the animal has had an attack of paralysis, or is likely to have one: at all events it clearly shows that either the brain or spinal cord is to a certain extent diseased, resulting in loss of motive power in the pasterns.

Treatment.-Feed the horse well, and give fifteen drops of the tincture of nux vomica three times in the day. If the hock joint be the cause, treat the hock as for spavin, or palliate the symptoms by applying two parts of olive oil, and one part of creosote and oil of turpentine, two to three times in the week.

Lameness.-Lameness occurs in many ways, and from many causes. Fractures, bruises, sprains, wounds or injuries. These will be found treated of under their various names through the book.

Laminitis.-A name in use by veterinary surgeons when speaking of founder, and is a generic term from lamina, or leaves, which form the bond of unity between the sensible and insensible structures of the horse's feet, and is the seat of the disease commonly called founder. (See Foot Diseases.)

Lampas.-This is a name applied to slight enlargement, swelling, or fulness of the bars of the mouth of young horses from the changes of teething. Pinching the skin of the bars with the nail of the thumb till they bleed, and rubbing in a little table salt, is much better than burning the mouth with a red hot iron from the ef- 
fects of which the animal never forgets nor forgives, whilst any attempt is being made to do any thing about his head.

Laryngitis.-This is a disease or inflammation of the upper portion of the wind pipe accompanied with fever, increased breathing, and cough. The cause and treatment of this disease will be the same as for bronchitis, (which see.)

\section{Leg, Fractures of.-(See Fractures.)}

Lice.-Lice of various kinds are often the source of much trouble amongst horses kept in the vicinity of hen or chicken houses.

Symptoms. - Uneasiness, rubbing, and scratching; stamping with the feet and biting at the legs, as if something were annoying him.

Treatment.-Take of the liver of sulphur, one ounce; cold water, one pint. Mix, and apply with a hard brush to a portion of the body at a time. If that is not effectual, get bichloride of mercury, thirty-two grains; cold water, one pint. Mix, and apply with a brush to a portion only at a time, or a piece of the body every day, till all has been gone over with the brush. This is very weak, and can do the horse no harm.

Ligaments.-These are strong, fibrous substances, which bind together the different bones of the body or skeleton. There are two great classes of ligaments; the rounded, or the lateral, and the capsular, or sack-like ligaments, as of the shoulder and hip-joint.

Lipoma.-A variety of fatty tumor. (See Tumors.)

Liver.-The liver is the largest secreting gland of the body, situated within the short ribs on the right side. 
Its function is the secretion of bile-a yellow alkaline or soapy fluid.

Without the liver, digestion and animal heat cannot be maintained, and the waste or effete matter cannot be removed from the blood. So, therefore, when the liver is disturbed, there can be no health in the rest of the system.

Liver, Inflammation of the.-The horse is rarely the subject of inflammation of this organ in an acute, but more commonly in a chronic form: it is often met with from the fact of many horses being highly fed, and having nothing to do.

Symptoms.-The affected part is very obtuse. But we have a very striking analogy of this disease in man and the horse, which materially assists in forming a correct opinion as to the disease. Pain and lameness in the right shoulder are characteristic of liver disease, whether in man or horse, and have been often mistaken and treated as for the disease itself. Not less so is the peculiar yellowness of the membranes of the eyes, nose, and mouth, constituting a disease called the yellows of the old horse doctors.

Treatment. - Give powdered aloes, four drachms; powdered ginger root, two drachms; podophyllin, one drachm. Mix, and make into a paste with molasses, and form a bolus, or crumble the mass in a little thin gruel, and drench the horse with it. Feed the horse with green and soft feed to keep his bowels open.

These measures being neglected, suppuration or an abscess will be formed, and break into the bowels, or become absorbed and produce glanders, which I believe to be a prolific cause of this disease, preceded by ill health 
and bad habit of body, terminating by a mysterious and unaccountable discharge from the nose, inasmuch as it is not accompanied with cough, and other symptoms of cold.

Locked Jaw.-This disease occurs usually after wounds of the feet, as from nails running into the feet, from wounds and fractures, and from a simple wound of a tendinous portion of the body. Locked jaw occurring after wounds or other injuries, is called traumatic. And when locked jaw takes place, as it sometimes does, without any injury or assignable cause, it is called idiopathic locked jaw. Locked jaw may be defined a spasmodic contraction of the muscles of the body, often confined to one set of muscles alone. I have in practice seen the same contraction in one set of muscles of the body, and the muscles of the jaw free from the cramps and not fixed at all, and depending upon the same causes as often produce fixedness of the jaw. Locked jaw is sometimes confined to the muscles of the neck, and is then called trismus.

Symptoms.-The symptoms accompanying locked jaw in the horse are so well known to every body, that little need be said by me about them further than that there is general stiffness and fixedness in the manner of standing, and a peculiar expression of countenance: the extended and dilated nostril, and the fixed ear, tell the tale so plainly, that the muscles of the head and neck are beyond the control of the animal, else his jaw or mouth would not be kept closed.

Treatment. - Remove the painfully-stricken animal into a place by himself, where he will have plenty of air, and no sound or sight to disturb him, and where no curious idler can enter. Place a bucket of cold, thin gruel 
where the horse can get at it, without an effort to himself to reach it. This is all the feed he will be likely enabled to take for a period of from three to sixteen days. Renew it once a day, and keep it sweet. $\mathrm{He}$ may be able to suck this through his teeth. Small, choice morsels of other food should also be placed within his reach, so as no opportunity be lost whereby his stomach may be filled, and his overtaxed strength be supported.

In securing the gruel or other feed, have every thing at hand, so that only one journey will be necessary in the twenty-four hours to the place he is confined in. Open not the door of his house twice when once can be made to answer: thus much suffering may be avoided, and the chances of recovery enhanced. All the medicine necessary for the horse to have, will be one drachm doses once in the twenty-four hours of PRUSSIC ACID. Great care will have to be exercised in keeping this powerful poison; and considerable judgment as to how this medicine is to be given to an animal with his jaws closed. Gextly elevate the head a little to insure proper gravitation, and pour the acid into the widest part between the teeth, and hold the head steadily for a few minutes; then retire, and close the door, not a loud word being spoken. A table or dessert spoon will answer for the purpose very well. Veterinary surgeons have an elastic tube, which is introduced into the back part of the mouth, and the acid poured down the tube.

If the animal live from three to four days, he may get well with such treatment, if we give him every opportunity to eat a little. Whatever wounds or injuries that have given rise to the lock jaw, should be dressed with equal portions of olive oil and creasote, which will soothe 
the irritated nerves of the part. An occasional poultice of flaxseed may be necessary.

Above all things neither bleed nor physick, as these can do no good, and will only hasten the death of the animal by taking away whatever strength he may have, all of which will be necessary to carry him through so severe a disease.

Loins.-Sprain of the loins or small of the back, is sometimes seen in weakly built horses of irritable disposition.

Symptom.-Weakness upon pressure, as of a saddle horse yielding to the rider, when mounting the horse.

Treatment.-Warm water cloths laid over the parts for a few days, followed in the same manner by cold-water cloths. Rest and good feed, and use the horse in the shafts of a light four-wheeled carriage, or in double harness. Saddle work may be the means of its return, more especially if ridden by a heavy person.

Loss of Appetite.-This is more of a symptom than a disease, and requires for its removal the cause which gave rise to it. In cold, influenza and fever, the appetite is bad, and with their removal the appetite will be restored. Powdered Carbonate of Ammonia, Pimenta Berries, Gentian root, each two drachms, mixed and given twice in the day, mixed in some cold gruel, and drench the animal. This will not only remove in a measure the cause, but will restore the appetite also.

Lumbago.-This is applied to a peculiar stiffness over the loins or back, partaking of the nature of rheumatism, (which see.)

Lungs.-These are the organs of breathing, and are 

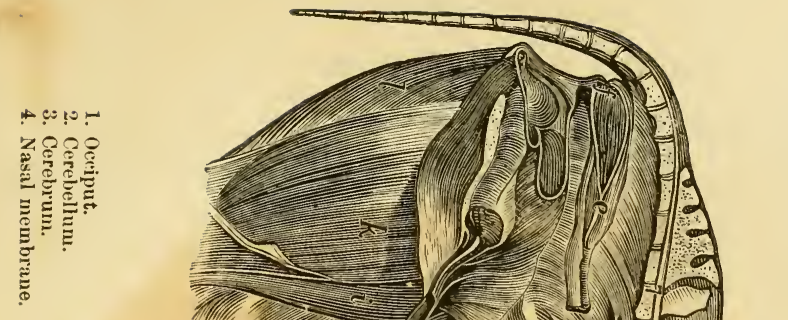

scos

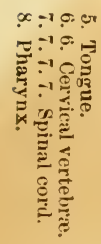

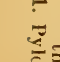

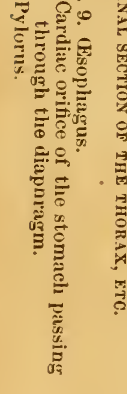

보ำฺ

트류

ํํำ

:

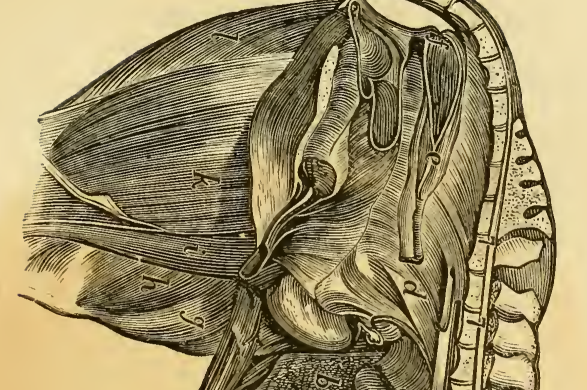

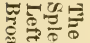

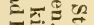

वृ.

है ڤ

。

ङ वे

ङ

离

苛

鸹施

क

竎娄

气

需

产

昰

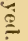

050

$\rightarrow=$

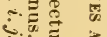

-

$\because+$

绨
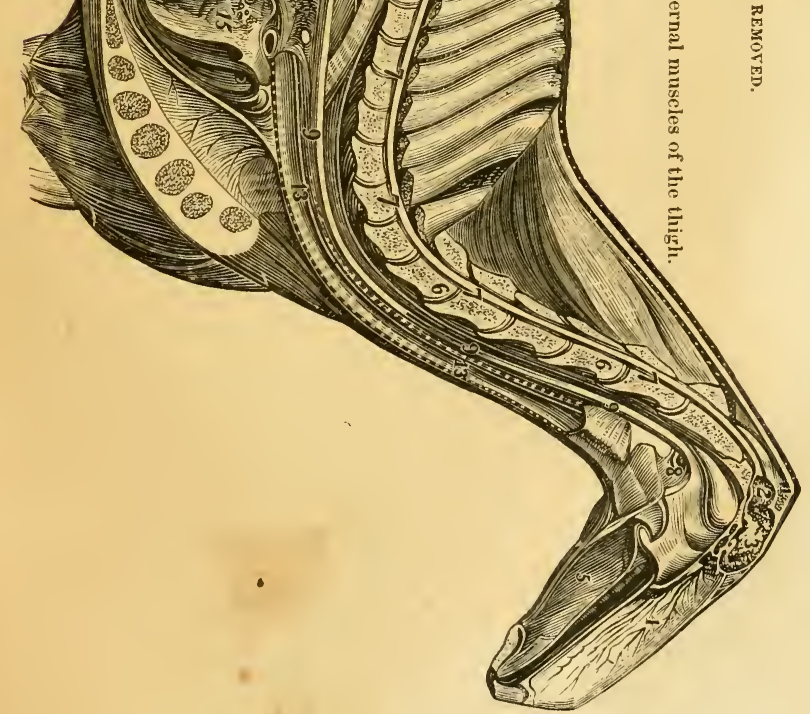

subject to many diseases, having names familiar to every horseman. Under this head, however, I will only notice inflammation of the substance of the lungs, pneumonia, (lung fever,) and of the surface of the lung, pleurisy, $a b$ scess, adhesion, and congestion. (See Cough, Cold, Bronchitis and Emphysema.)

(1.)-Pneumonia.-(Pronounced numonia.) - This is an inflammation of the substance of the lungs, of late years called lung fever, and is sometimes very common in the spring after a severe winter.

Symptoms.-Chill followed by fever and increased for a short time, and is succeeded by cold legs and ears, quickened breathing, and wide, open nostrils. A peculiar quivering of the muscles of the side and breast will be observed in all cases of inflammation of the lungs, and will rarely deceive. The animal will eat nothing, and persistently stands with his nose and mouth in the manger; and if taken out of the stall and stable to the open air, he will almost refuse to go back again into the stable, relief being experienced from the fresh air. Hence, the necessity for plenty of fresh air in all diseases of the lungs. When the ear is applied to the side of the neck, a peculiar creaking noise is heard. Slight discharge of serous flakes or matter will be observed sticking to the sides of the nose. If this disease be not subdued or cured, it ends in abscess. The peculiar pulsations observed in this and other diseases, will be found treated of in the introductory remarks. To the non-professional person, the general aspect or appearance of the horse, when under this disease, is of infinite more importance as a rule and guide in determining the seat and nature of the disease, than from any assistance they can gain from the pulse. 
Cause.-Alterations and sudden changes in heat, cold, and moisture. An animal not in condition for work. A hereditary predisposition in the lungs to take on disease. Changes which will produce lung disease in one horse, will produce disease of a different character in another.

Treatment.-Place the horse in a light and airy place, and clothe him according to the weather. Bandages to the legs will at all seasons be necessary to keep them warm, and to that extent relieve the lungs of a portion of blood. Aconite judiciously given, is the most powerful remedy I am acquainted with. Tartar Emetic, so valuable in this disease in man, dogs and swine, has no effect whatever, upon either horses, sheep or cattle, than so much flour or meal. Then give twenty-five drops of the tincture of aconite root, in a cupfull of cold water, and drench the horse. Repeat the dose every four hours, till six doses are given. In the majority of cases, one to two doses will be all that is required to perfect the cure.

(2) Pleurisy.-Inflammation of the membrane covering the lungs and lining the cavity of the chest.

Symptom.-A rigor or chill, fever, disinclination to turn short, an occasional short painful cough, and careful breathing, accompanied with a sigh or grunt. A peculiar line will be observed in pleurisy, running from the haunch, round the belly to the breast-bone. The breathing is deep, not so short and quick as in inflammation of the lungs. In the first twenty-four hours from the attack, pain will have given way, and the horse be apparently better. This in the majority of cases of pleurisy, when left a few hours to itself and not checked, terminates in Hydrothorax, (which see, or water or serum in the 
chest, sometimes causing adhesions. The favourable termination of pleurisy is by what is called resolution.

Canses.-Changes in the atmosphere. Exposure to cold. Broken ribs or wounds.

Treatment.-Treat the horse as for inflammation of the lungs, by pure air; cold water and aconite followed on the second day by five grains of powdered spanish fly in gruel, once in the twenty-four hours. To remove the fluids from the body, give after the active stage of the disease has passed, good feeding and generous diet.

(3.) Abscess.-As elsewhere stated, abscess is the termination of inflammation of the lungs. Pus is a common result of inflammatory action, and when in the lungs is called vomicae, causing in some cases consumption, and where absorption of the pus has taken place, glanders is the result.

The abscess frequently breaks into the bronchial tubes and then pus escapes into the larynx and nares of the nose; hence the persistent discharge which is so characteristic of glanders.

Treatment.-The same as for glanders, generous diet, tonics and stimulants, with soda sulphite and the Spanish fly. (See Glanders.)

(4.) Effusion of Serum.-Hydrothorax is one of the ways in which pleurisy terminates, and when this effusion is extensive, not much hope of recovery may be expected. Cures, however, have been made by drawing off the fluid by means of a trocar piercing through between the ribs into the chest.

(5.) AdHesions, - The surface of the lungs become attached to the sides of the chest, by fibrous bands of great strength, another common result of pleurisy. Nothing 
can be done but to keep up the health and strength by good feeding, etc.

When animals do not thrive and pick up their spirits and flesh after cases of pleurisy, some of these various conditions may be reasonably expected to be present.

(6.) Congestion of $\mathrm{THE}_{\text {- }}$ - The lungs are liable to become congested, when they are over charged with blood.

Symptoms.-The horse blows, his nostrils are very much expanded, heaving at the flanks. The horse is the picture of distress and stupidity.

Cause.-Weakness and want of power in the blood vessels to contract and empty themselves.

Treatment.-Allow free access to cool air, and clothe the body and bandage the legs to encourage the blood to the skin and legs, and give the following mixture; Sweet Spirits of nitre, half an ounce; Powdered Carbonate of Ammonia, half an ounce: mix in a bottle of cold gruel in the form of a drink. If these articles are not at hand, give two bottles of warm ale, or a half bottle of brandy or whiskey.

(7.) Pneumothorax.-So called because the air escapes into the cavities of the pleura.

Lymph.-Is a name applied to the clear fluid which circulates in the lymphatic vessels and that which is poured out in cut surfaces, after bleeding has stopped, and forms the medium by which the parts adhere, and are joined together. In this instance it is termed coagulable lymph, the principal element by which adhesive inflammation is carried on.

Madness.-A disease produced by the bite of a mad dog. (See Hydrophobia.) 
Mad Staggers.-An affection of the brain. (See Staggers.)

Maggots.-These are sometimes seen in neglected wounds and sores, in warm weather. To remove them, apply equal parts of creosote and olive oil, or a solution of corrosive sublimate.

Malignant. - A term applied to diseases of a fatal character; as glanders, for instance. Why the term malignant should not be applied to such a disease as lockedjaw, or inflammation of the bowels, which are so often fatal, is one of the inexplicables of medical nosology or terminology, not easily for me to understand.

Malignant Epidemic.-English writers tell us that a malignant epidemic has attacked horses on the European Continent. Influenza is a disease from which scarcely one per cent should die when scientifically and intelligently treated; but by bleeding, blistering, physicking, and low diet, a really simple and non-fatal disease, is at once converted into a fatal and malignant epidemic.

Mallenders.-A term used by old books and horse doctors, to a scaly condition of the skin of the back part of the leg, opposite to the knee. A term which certainly, to say the least, should long ago have been blotted out of all the books, as vague, uncertain, unmeaning. It is sad, to think of the low condition of the mind of the genius horse doctor.

This scaly eruption is the result of dryness of the skin of the back part of the leg, where the greatest and almost constant movement of the joint is going on. The same condition is seen on the face of some joints, and in others the back, from the constant mobility of the parts. 
Who has not seen scruffy or scaly heels of horses ending with scratches? (See Skin Diseases.)

Mange.-Is a disease of the skin, and is caused by a small mite called acari, which breed and burrow in the skin. To cure mange, destroy the insect. (See Skin Diseases.)

Materia Medica. - This is a name used or applied to every substance used in the treatment and cure of disease.

Megrims.-A disease of the brain occurring at periods, especially in hot weather, and when exposed to a powerful-sun. This differs from epilepsy only in the absence of spasms. (See Epilepsy.)

Causes.-Tumours in the choroid plexus, and enlargement of the pineal gland.

Treatment.-Merely palliation, by using a dutch collar, so as not to interfere with the circulation of the blood from the head.

Observe.-In summer, the horses subject to brain disease, or fits of any kind, should not be used for family purposes. In winter they will make useful animals.

Melanosis.-A variety of cancer peculiar to gray horses, which turns white with age, and is caused by the transfer of the coloring pigment from the skin to the blood. (See Cancer.)

\section{Melanoid.-(See Cancer and Tumours.)}

Mesentery.-A membrane formed of two folds of the peritoneum, and between each there are numerous glands, lacteals, lymphatics, arteries, veins and nerves. It is called the mesentery, because it adheres to three lumbar vertebræ, and has the small intestines hanging to it. One portion of it is called the mesocolon-supporter 
of the colon, and another the mesorectum, which encloses the rectum.

Mesenterica.-Wasting of the mesentery is a disease which is not very common to horses, although some clearly marked cases are sometimes seen, characterized by wasting of the body, weakness, and general debility of the whole system. Bowels irregular:-sometimes soft, at other times hard, and of a pale or straw color, and frequently of bad smell. All that can be done is to keep the strength and condition of the horse up by iron and gentian, which is so often recommended throughout the book.

Metastasis.-A term denoting a change or shifting of aisease from one part of the body to another, which is so well illustrated in cases of rheumatism.

Moon Blindness.-(See Eye Diseases.)

Mortification.-Death of a part. (See Gangrene.)

Moribund.-A term in use, and applied when man or animals are in a dying condition.

Mouth Diseases Of.-These are but few; perhaps the irregularities of the teeth are the most important; so much is this the case, that from diseased or carious teeth, an affection, to many external appearances, is similar to glanders; and horses have accordingly been destroyed, whereas, if a carious tooth, producing a stinking discharge from the nose had been removed, these appearances would have passed off. The edges of the teeth of horses, at all ages, are apt to become sharp, and cut or wound the inside of the mouth, and interfere with mastication or chewing. When horses are off of their feed and losing flesh, it will be well to have the teeth examined. 
To remedy any irregularity of the grinders, a rasp or file, with a concave surface and long handle, is used to make

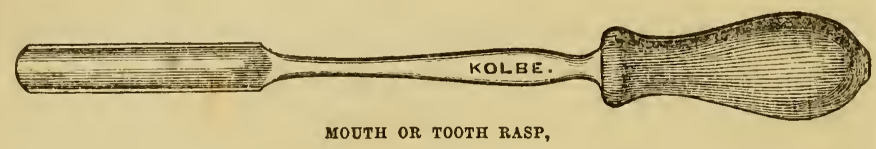

the teeth smooth and level. Wolf teeth are supernumerary, but do no injury to either the mouth or the eyes.

1. Scald Mouth.-Another simple affection of the mouth, and is characterized by the horse slobbering or frothing from the mouth, as if salivated. In aggravated cases fever is present.

Treatment.-Give ten drops of the tincture of aconite root in a little cold water three times in the day, for 48 hours, and allow the horse to have a bucket of cold water suspended or placed before him, to cool his mouth in.

2. Wounds of the Tongue-Will be treated the same way, but without the aconite. If the tongue is nearly cut through, have the cut portion entirely removed. 'The horse can do wonderfully well without a large part of his tongue.

3. Black Tongue.-This is not a disease, but the effects of a simple and non-fatal affection, treated by bleeding, blistering and physicking, destroying vitality, and inducing mortification of the tongue, as well as of other portions of the body.

4. Thrush.-Soreness of the mouth, with white patches on the tongue, inside the cheeks and roof of the mouth. In man, this condition of the mouth is called stomatitis.

Causes.-Bad condition of the stomach and dyspepsia.

Treatment.-Borax in powder, one ounce; molasses, three ounces; mix, and apply with a soft brush, or soft 
piece of cloth. Give soft feed or cut grass. A few doses of sulphite of soda, half an ounce to a dose, given for a few evenings, will be all that is wanted. (See Lampas.)

The mouth is a favourite and convenient place for horsemen to try the keenness of their pocket knives, when the least pretext is offered.

The palatial artery is sometimes cut lengthwise; and when that is the case, the bleeding thus unnecessarily induced, will not stop when it is wanted. Many plans and contrivances are recommended by individuals, how to stop such bleeding; but none are equal to a piece of iron or kitchen-poker immersed for a few minutes in hot water, and applied to the wound for a moment, which will at once stop further loss of blood.

Mucus.-A thick, viscid substance, thrown out from the mucous membrane, throughout the body.

Mucous Membrane.-A thin lining of all the air passages; so-called, because the surface is kept moist, with a slimy matter, as referred to in the article abov e When this mucos is altered in quantity and quality, and when mucous-pus is poured out, disease is present. This is seen in cases of cold, bronchitis and inflammation of the eyes, (which see.)

Myalgia.-A term given to inflammation of a set of muscles, and is applied by some, to wasting of the muscles, as is sometimes seen in sweenie, (which see.)

Narcotics are medicines which act upon the nervous system, diminishing its power and sensibility, and so relieving inflammation, irritation, and pain. A medicine capable of doing this also produces the properties of an anodyne, a sedative, soporific, carminative, and nauseant.

There are but few medicines possessing this power over 
the horse, and these are, aconite, prussic acid, veratrum, and perhaps lobelia. In my practice, nothing answers the purpose so well as aconite. It is the great antiphlogistic. Indeed, so great is its power, that in bronchitis, inflammation of the lungs, feet, and bowels, or where there is pain and fever, no remedy or remedies can compare with this invaluable medicine in the treatment of diseases of the horse. (See Medicines and Prescrip-tions.)

Nasal Gleet.-A thin, transparent discharge from the nose. (See Gleet.)

Navicular Disease.-This is a disease commonly called coffin-joint lameness, and by some it is termed grogginess. (See Foot Diseases.)

Necrosis.-This is a term given to a dead bone when it is attached to a sound one. The difference between caries and necrosis is this:-Caries is present when the bone is impaired only, and necrosis when the bone is entirely dead, and its functions have entirely ceased. When a bone has fallen into the condition of necrosis, its removal becomes as necessary as the removal of any other dead or foreign matter in order that reparation and restoration of the function be repaired, and a cure be made.

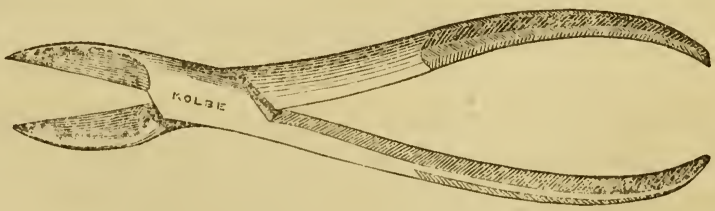

USTON'S BONE FORCEPS.

Nephritis.-A technical term applied to inflammation of the kidneys, (which see.) 
Nervousness. - Few persons having the care of horses have failed to observe occasionally horses with a peculiar excitability of disposition, when any excitement and noise is going on, and when beir:g harnessed for work. The tail somewhat elevated, moving from one side of the stall to the other, and passing manure from him repeatedly every few minutes, until one would think that there was nothing left in his bowels. These animals are usually light-bellied and poor feeders, but fleet and free goers, and very gay in saddle or har ness, and much admired by persons not versed in horseflesh. They make excellent Sunday horses, but poor every day animals, as the constant excitement when at work overdoes their physical powers. This condition impairs the value of the horse very much.

Treatment.-Keep nervous horses in a place by themselves, where there is no noise or sound to disturb them, and have no harness or saddles in the place with them, nor clean harness or saddles where they are; for whenever a piece of harness is seen in the hands of the groom, the animal expects it is to be put upon him, and hence he gets excited, and efforts are made to empty the bowels of their contents. The harnessing or saddling should be the last thing that should be done before going out with such a horse, as it gives him no time to empty the bowels and become excited. Ten grains of opium, and a drachm or two of prepared chalk may be given, either half an hour before going out, or after he comes in. Such horses are more pleasant to drive, if this be given.

Neurotomy. - An operation for dividing the nerves of feeling, as they enter the foot on both sides of the leg. The operation is performed for the purpose of removing pain from the foot in navicular disease. The 
operation, however, has of late years fallen into disrepute on account of ignorant men operating indiscriminately on feet of all forms and shapes alike; and, consequently, no surprise should be expressed, when in some of these cases the feet ultimately fall off.

Neurotomy should never be performed upon flat and weak-footed horses, as they are easily bruised and suppuration is set up, and separation of the outer and inner foot structures. Weak and flat-footed horses when sound, are careful how they put their feet upon hard roads and paved streets; but when deprived of all feeling by the operation of neurotomy, they let their feet come down on the ground with great force, so as to injure the foot, resulting, as before stated, by the hoof falling off.

Nose, Diseases of.-(See Cold, Catarrh, and Bronchitis.)

Numbness.-Loss of feeling in any part, usually indicating disease of the brain, resulting in paralysis or palsy.

Obesity.-This is a term applied to morbid or unhealthy fatness. When this condition is in the mesentary, it produces big belly; in the liver, fatty liver; in the heart, fatty degeneration of that organ.

Causes.-Little or no work, or exercise disproportionate between the amount of food taken and the waste.

Treatment.-Constant and regular work; feed in small bulk oats instead of corn, and not much hay. To animals inclining to take on too much fat and flesh, give a dose of physick (see Aloes, Medicines and Prescriptions) occasionally, but do not bleed.

CEdema.-A term signifying soft and not inflammatory swellings of various parts of the body, as a sequel 
to debilitating diseases. These swellings contain serum thrown out from the blood. The treatment of this affection will be the removal of the exciting cause. (See Dropsy.)

CEstromania.-This name is by some called ostening, which name is applied to mares and cows when desiring the male.

Omentum.-A fold of the peritoneum, which hangs down from the stomach, and is reflected on itself upwards and backwards to the colon. It is in this where the great deposition of fat takes place. The omentum is often implicated in rupture.

-Open Joints.-(See Broken Knees.)

Opthalmia.-(See Eye Diseases.)

Ossification.-The formation of bone; but in the language of medical men, it means a deposition of earthy matter in the soft textures of the body where bone does not exist. Thus, for instance, we speak of ossification of the lateral cartiliges of the foot, which form ringbone. We have ossification of the heart, arteries, and of other parts of the body.

Osteology.-A name used in speaking of the bony system.

Osteoporosis.-This is a name given to big head, (which see.)

\section{Ostitis.-(See Splint.)}

Overreach. - This is the consequence of driving faster than the horse can go. The injury is generally done by the edge of the inner rim of the shoe. Avoid the cause, and treat the wound with the simple ointment. (See Prescriptions and Medicines.) 


\section{Ozena.-(See Gleet.)}

Palliatives.-Medicines given not so cure disease but to relieve the symptoms of pain.

Paralysis-Palsy.-Loss of the power of moving in some parts of the body. Paralysis may be confined to one leg or two legs, then it is called partial. When the horse has lost the power of standing, and the four legs are affected, then it is complete. Usually however in the horse it is confined to the hind parts, or the haunches and legs. Sometimes the paralysed part is numb, at others the sense of feeling remain.

Causes.-Disease in the brain and spinal cord.

Treatment.-If the patient is young, exercise patience and time, and nature will do a great deal in a disease of this kind. The general health is to be kept up by good feeding and tonic medicine, as fifteen drops of the tincture of nux vomica, four ${ }_{E}^{\mathrm{E}}$ times in the twentyfour hours. Turn the horse from side to side twice in the day, and give plenty of dry clean bedding, to prevent the skin from scalding and peeling off which is sometimes a source of great irritation to the poor horse. The paralysed parts should be well rubbed with a stiff brush. Electricity has been thought of advantage in this disease, but from what I have seen, not much need be expected from it. The nux vomica offers with good feeding and care, the best chance for recovery. It must be remembered that this is a nervous affection, and probably these diseases are not so managable nor are they so easily cured as other affections of a different type.

Parotid Duct, Distended.-This is a rare affection 
in horses, it resembles a round ball attached to the edge of the lower jaw, an elastic encysted tumour or rather like an encysted tumour. When I was called in to see a horse, which proved to be so effected, and telling the gentlemen that so long as the ball did not break, it would do the horse no injury, and that I would not recommend its removal, he said he would give five hundred dollars if the horse had another on the other side, then he would look like an Angora goat.

Parotid Duct-Open and Fistulous.-This is a serious affection, for every movement of the jaw in chewing or masticating the feed the glands pour out the saliva which should mix with the feed, and assist in the act of digestion, but on the contrary is poured upon the ground and is thus lost. The animal becomes thin of flesh, gets weak and after a time dies a miserable object.

Causes.-Injuries, or accident to the gland, or its duct resulting in suppuration and from the mobility of the parts, fistula is established.

Treatment.-Few horse doctors or farmers can cure this affection. An expert or accomplished surgeon is only able to effect a cure, and this will be by closing the open or fistulous duct so that the saliva will with the feed find its way into the stomach.

India rubber dissolved in chloroform, applied over the mouth of the wound, when it is thoroughly dry will stop it for a few days, and by continuing this application, a cure in a very many cases can be made.

Pathology.-A department of medical science, which treats of the causes of disease, and of the appearances of diseased parts when living or dead. 
Patella, Dislocation of.-This is a common occurrence in high spirited, nervous and weakly horses.

Symptom.-The horse stops, if at work, and throws up his head, with the pastern of the dislocated leg, slightly bent, and the leg held back behind the body and is unable to bring it under it. Fever and irritation sometimes accompanies this accident, more especially if it is of rare occurrence in that animal. The oftner the patella has been out the less fever and irritation will be seen. There are horses with which its of frequent occurrence, and happily for them, there is just as little trouble in putting the leg in its place again, a crack of the whip will do it sometimes. This is a serious object, in an otherwise fancy horse. There is but one other affection of the hind leg which can be mistaken for it, and that is cramp. (Which See.).

Treatment.-Remove the horse to a stable, and attach a rope to the pastern of the leg which is dislocated, and carry the end of the rope through a ring or over a beam at or about the horse's head, and place the end of the rope in the hand of one or two strong men, telling them not to pull till a man is placed at the head of the horse to keep him steady, and another man at the leg with one hand placed firmly on the point of the hock joint pulling towards himself, with the other pushing firmly against the dislocated joint, then the men on the rope will pull firmly and gently, till the foot is brought fair in under the horse's body, after which the rope is to be removed and the horse kept quiet for a day or two.

Pasterns.-(See Sprains.)

Pelvis.-The anotamical name for the lower part of the abdomen or belly. 
Pelvic Abscess.-This condition is sometimes seen in weakly constitutioned mares within a few days after foaling.

Symptom.-In from one to four days one of the thighs of the hind legs will be swollen, hot and painful, causing the mare to shiver or appear chilly, not from cold but from the suppurative inflammatory action going on. The milk will have almost entirely ceased to be, and the colt will have to be fed by the bottle as a child, or out of a bucket like a calf, till the mare is cured and the milk returns. One curious condition about pelvic abscess although it suppurates the abscess does not break usually on the thigh as it would be expected to do, but it breaks within an inch or two from the haunch bone.

Treatment.-The pus which has accumulated from so large an abscess, does not discharge itself from the place of opening, but burrows away down among the muscles of the hip and thigh, down to within a few inches of the hock itself. IIence the great secret in the treatment of pelvic abscess is to make two free openings, one above at the point of the soft abscess, and the other from four to six inches of the hock on the outside of the thigh. Then take a smooth elastic twig or a long piece of whalebone nicely smoothed with sand-paper, and introduce into the opening above, and gently force it down to within an inch or so of the lower opening. This being done the pus will all discharge itself from the lower hole, then inject with a small syringe once a day, for a few days about half a tablespoonful of the following mixture: oil of turpentine and olive oil, equal parts. Keep the parts clean, and feed the mare and colt well, and leave nature to complete the cure. 
Penis Hanging out.-This a serious defect, and is the result of weakness and debility. The organs of generation are weak, the sheath swells clasping the penis in its grasp, and it also becomes enlarged and in most cases never will be able to draw it into its place again, so it will either have to remain so or have it cut off close to the prepuse or sheath. This affection and the opposite condition, Phymosis and Paraphymosis was very prevalant amongst the horses of the army, in the late war.

Peristaltic.-A term applied to the serpentine motion of the bowels, one portion contracting and forcing its contents onwards into the next.

Peritonitis.-Inflammation of the peritoneum or the serous membrane, which lines the walls or inside of the belly, characterized by great pain, and is the result of accidents or injuries and from surgical operations.

Treatment.-The same as for any disease of an exalted kind by using aconite root, cold water and pure air, and after the pain and fever have subsided, good feeding.

Periosteum.-The thin pearly covering investing the bone. It is the stretch of this membrane in cases of splint which cause pain and lameness. (See Splint.)

Phagadena.-A named used in surgery, implying a spreading and destructive ulcer, which spreads rapidly and destroys the surrounding parts. The true meaning of this word is eating, and in its effects are similar to what is called in domestic practice, hospital gangrenea local, spontaneous combustion, in which oil globules are poured out in great quantity in and around the sore or ulcer. In horses these ulcers are common on the 
heels and legs of horses after a severe winter. When on the heels, the ulcers are taken by horsemen to be scratches. Phagadena does not usually assume the form or appearance of a cut or scratch, but is generally a flat, round or oval, and circumscribed sore at first; the hair of the part is standing on end, with oil drops all over the surface, and in a few days the whole of the skin and hair falls off, or a separation of the edges of the sore will take place, and the skin and flesh of the part will completely fall out, (called core) leaving an unhealthy looking sore, with a white sanious fluid covering the whole of its surface. When the slough does not take place, it is gradually eaten away, and in this case leaves on the edges and surface of the sore a thin dirty colored looking skin or membrane.

Causes.-Bad habit of body, from impure blood, death of the part from exposure of the heels in some mixture of salt and snow. Cold drafts under stable doors.

Treatment.-The complete removal of all dead matter belonging to the ulcer, and a thorough cleansing of its inner surface. This is important as it will not only be important as a measure of cure, but as a surety against it spreading further up the leg or heels. Then sprinkle the edges and inner surface twice in the day for a day or two with powdered blue stone to destroy the unhealthy surface, and hasten a red surface or the granulatory process by which the hole will be speedily filled up again. Complete the cure by sprinkling over the sore with powdered loaf sugar twice in the twenty-four hours. Support the strength of the horse by good and generous diet. (See Scratches.) 
Phlebitis.-A name given to inflammation of the vein after bleeding characterized by swelling and cording of the vein. (See Jugular Vein.)

Phlegmasia Dolens.-A name given to one leg when swelled to a great extent from plugging of the blood vessels of the leg with plastic matter poured into them from the blood. (See Grease.)

Phrenitis.-One of the many diseases of the brain characterized by the horse becoming unmanageable-a variety of staggers, or it may be of inflammation of the brain itself, and is incurable.

Physiology.-A branch of medical science treating of the life and functions of organized bodies.

Physicking.-In England and in some parts of the European continent, a person who does not know how to physick a horse, whether sick or well, is not considered fit to take care of horses. In the United States, the man who knows all about, and recommends physicking under almost any circumstance, should not be permitted to even to take a horse by the head, much less to take care of horses not his own. In an extensive practice of many years, I do not recollect a half dozen times, when I either gave or recommended a horse to be physicked; and no man can show equal success in the treatment of disease of horses and cattle, let his mode of practice be what it may. Remember, when the bowels are opened, and emptied of their contents, an important pillar has been taken from under the animal structure. Show me an European book on the diseases of animals, which does not recommend and minutely describe how to physick a horse, and I will show you a city without walls, 
without a church, or gymnasium. In this connection, I am sorry to say, that our American authors on animal diseases, have copied too closely from European practice, which is utterly unfit for this country, climate and the constitution of all our domestic animals.

Pleurisy.-Inflammation of the serous membrane covering the lungs, and lining the sides of the chest. (See Lung Diseases.)

Pleuro-pneumonia.-Inflammation of the covering and substance of the lungs.

Pleurodynia.-This is a rheumatic affection of the intercostal muscles, and differing from pleurisy, from there being no constitutional disturbance, little fever, and no inflammation, and is treated with a dose or two of the tincture of aconite root given internally, and mustard and a little vinegar rubbed into the muscles of the sides, behind the shoulder.

Plethora.-Fulness of blood. (See Obesity.)

Pneumonia.-Inflammation of the lungs, (which see.)

\section{Poisons.}

Poisons act in a variety of ways in destroying life, and are derived from the organic and inorganic kingdoms, and their effects are either local or remote. Poisons may be taken into the stomach, inhaled in the form of sulphuretted hydrogen, and from the skin as from the bite of a poisonous animal, or absorbed from wounds. (See Glanders.) Poisons act in one of three ways:

1.) Irritant Poisons are those poisons the symptoms of which are inflammation, irritation, and pain. Examples-arsenic, bi-chloride of mercury, lead, baryta, copper, and the Spanish fly. 
Treatment.-The plan to be adopted in this class of poisons in the horse, will be by giving large quantities of the white of eggs, milk, linseed oil; and remove the poison as speedily as possible by giving large quantities of linseed oil, say two quarts. The horse cannot vomit; hence, there is a difficulty in a prompt evacuation of the stomach. If the pain be great, give aconite to subdue it, and to keep down inflammation and sympathetic fever.

(2.) Narcotic Poisons.-Poisons which act on the brain and nervous centres, producing stupidity or coma.

Treatment.-Give four grains of strychnia nux vomica in a few pints of gruel made with vinegar. Keep the horse walking around, and place chopped ice in a bag, and put it on the forehead.

(3.) Narcotic Acid Porsons.-Poisons acting as the above, and causing irritation, inflammation, fever, and pain. Examples of this class are, nux vomica and veratria.

Treatment.-Aconite will not only relieve the pain, but is an excellent antidote for strychnia, and for aconite, strychnia may be given with advantage in cases of this variety of poisoning.

Poisoning from lead and copper is most frequent in the country, and in the vicinity of lead and coppersmelting works, and in pastures where manure from large towns and cities is spread, and on farms where the water is conveyed in leaden pipes, and kept in troughs and cisterns lined with lead. Pieces of lime and nails, or scraps of iron finding their way into leaden troughs, cause oxidation of the lead, forming sugar of lead,-a bad poison. Not long since, heavy damages were awarded to a farmer who had lost several head of cows from lead poisoning, from the spray of leaden bullets shot against 
a stone wall by a rifle, or military company. The fringes of lead spread upon the grass, was left to be converted into the sugar, or oxide of that metal, and the cows gathered it with the pasture.

Treatment.-Give large doses of white of eggs, and linseed oil, in either lead or copper poisoning, to shield the coats of the stomach and bowels, and to remove it from the body altogether. Happily for the horse and his owner, large quantities of poisonous materials are necessary to destroy life, and materials which will destroy man, dog, and the pig, will not in many instances have any effect on the horse, sheep, and cattle. Antimony, an active and deadly poison, when given to omnivorous animals, have no more effect in a poisonous point of view than the same quantity of earth, when given to herbivorous animals. Hence, tartar emetic is now no longer used as a nauseant in the treatment of horses and cattle, when laboring under lung diseases, however useful it is in the same diseases in man and the dog.

Poll Evil.-This affection of the back part of the head is well known to horsemen, without much of a description being given. It consists in supurative inflammation forming pus in the form of a simple abscess, or in the form of fistula, (which see.)

Cause.-Injury to the part, or disease of the bone.

Treatment.-As soon as the swelling has become a little soft, have it opened without delay, before the pus has time to burrow down among the bones of the neck, and cause disease in them. Make the opening large and deep enough, so as to admit three fingers, so that the abscess can be swabbed out with a piece of sponge or cloth tied on the end of a stick, to remove the pus. This 
will have to be done twice in the day, till no more pus can be brought out.

Occasionally syringe or squirt cold water into the sore, and swab it out again, till completely dry. Then apply the following once in a day with a swab previously dipped in it:-Creosote, one ounce; oil of olives, two ounces; oil of turpentine, one ounce; mix. In applying the mixture, do not use the swab too freely, not to break down the granulations or the healing processes that are springing up to fill the hole or cavity, so as to form a perfect cure.

When poll evil is the result of diseased bone, and partakes of a fistulous character, it will not be so easily healed or cured, for the underlaying bone is carious, and becoming necrosed, and before exfoliation or separation of the dead bone takes place, the horse may be dead, for the process may take years to perfect itself. And when it is cured, there is usually a stiff neck remaining ever after. Ten drops of sulphuric acid poured in the fistulous opening of the swelling or sore, will hasten recovery very much, and in many cases make a good and speedy cure. Once a day will be often enough, and if there be more than one fistulous opening, drop the acid into one to-day and the other to-morrow, and continue from day to day, till each and every opening ceases to discharge a whitish gray matter, and a dry looking opening is presented. After which, use a solution of the sulphate of zinc. One drachm of the zinc to four ounces of water, will answer the purpose. Horses having sores of all kinds on their body, should be well fed and cared for.

Polypi.-These are diseased enlargements, which grow from the mucous membranes of the nose, and ear, and in the uterus or womb of mares. 
Treatment.-If they are small, they are cured by touching them with a stick of caustic potassa; and when large, cut them off with a sharp knife or scissors, and apply a weak solution of blue stone to the sore till it is healed.

Predisposing Causes.-Are those which render an animal susceptible to disease. For example, a young horse standing in the stable from day to day, predisposes him to disease of the throat and lungs, when he is put to any exertion. Old age is a predisposing cause of disease. Some animals, as well as men, are more disposed to disease than others, from their temperament, and certain conditions of the solids and fluids of the body, make the body more susceptible to what is called a predisposing cause.

\section{Prick of the Foot.-(See Foot Diseases.)}

Procidenta.-A term used to denote the falling down of the womb, or foal bed in brood mares. This affection is very common in milch cows; not so in mares.

Prognosis.-Is the knowledge of foretelling the event of a disease, from its symptoms. Prognosis may be favorable or unfavorable. Nothing can so well distinguish the scientific veterinary surgeon from the blockhead in such matters as correct prognosis.

Prophylactics.-Is a term applied to the means made, or adopted, for the preservation of health, and the prevention of disease.

Proud Flesh.-A common name applied to hasty granulations in a sore or wound, which present a fungous appearance. To cure and prevent this, sprinkle a little white sugar, powdered blue stone, or a little red precipitate on the surface. 
Prurigo.-An itchiness of the skin, and is best treated by the sulphite of soda, in half ounce doses, given every night in cut feed for a week. (See Skin Diseases.)

Pulse.-This is the stroke or beat of an artery, consequent upon the alternate dilation and contraction of the artery caused by the action of the heart. The pulse is subject to many variations, even not depending upon disease. The pulse is also liable to changes from temporary excitement, as from severe heat, etc. Medicines act upon the circulation, and consequently change the beat and character of the pulse. If the disease be debility, diffusable stimulants will be required to raise the pulse, and in order to depress the circulation, as in inflammation and fever, nauseants are indicated, such as aconite and veratrum. (See Pulse, in Introductory Remarks.)

Puncture.-Wounds inflicted with a sharp-pointed tool, as a stable or hay fork, etc. Punctured wounds are dangerous, depending as to their depth and locality, and should be treated by free openings from the bottom of the wound, to allow the exposed fluids to escape, and dress the parts with simple ointment. (See Medicines and Prescriptions.)

Purgatives.-Are a class of medicines capable of cleansing or emptying the bowels. Purgatives are distinguished from laxatives, only in the quantity given. Six to eight drachms of aloes will act as a purgative, and two to three drachms will act as a laxative. When laxatives are necessary, repeat at distant intervals.

Purpura.-This is a disease which is but rarely seen, and consists in the surface of the whole body and legs being covered with pimples, or small boils, which discharge 
a livid, or purple-color fluid. The animal is very much debilitated, and accompanied with sympathetic fever, not unfrequently, in addition we see swellings of the head, parts of the body, and the legs very thick, and oozing out of them, the same colored fluid. The horse scarcely able to move. The worst form of this disease, is the purpura hæmoragica, or bleeding purpura. The small boils are the result of extravasation from the minute blood vessels under the skin.

Cause.-Venous congestion of the whole surface of the body, and possibly, a deterioration of the blood itself.

Treatment.-Support the strength, to keep off typhoid symptoms, and enrich the blood, and attend to the surface sores. For this purpose, give the following powders, night and morning:-powdered sulphate of iron, three ounces; gentian root, two ounces and a half; carbonate of ammonia, four ounces. Mix, and divide into twelve powders one to be given twice in the dav. Give occasionally forty to sixty drops of commercial sulphuric acid in a bucket of cold water. Feed the horse well; and apply to the sores olive oil, three ounces, and creosote, one ounce, once every second day, and wash the sores twice a week. Horses once attacked by this disease, are ever after liable to it again, as the blood vessels are so weakened, and generally remain so.

Pus.-This is the material found in abscesses. Pus may be healthy, or laudable, as it is called. Unhealthy when it is mixed with blood, and has a stinking smell. Healthy pus is of the thickness and color of cream, and is insoluable in water, and has no smell.

Putrefaction.-Certain diseases are regarded as putrid, where the discharges have a black appearance, 
and putrid smell. When weakness and debility are present, putrid ulcers spread rapidly. The treatment of putrefaction in a living animal should be directed to laying open the sores, so as to get rid of the putrid discharge before it is absorbed into the circulation, and immediately wash with the solution of the chloride of lime, and afterwards dress the sores with equal parts of olive oil and creosote, and sprinkle the sores with powdered charcoal. Give the horse good feed to support the strength, and give sulphate of iron and gentian root, two drachms each, night and morning.

Pyemia.-This is a term signifying pus in the blood, acting and setting up a fermentation in the blood. Examples, tubercles in the lungs, glanders, farcy, and grease. (All of which see. Also see Ferments.)

Quack Medicines.-Are medicines prepared according to private or secret receipts, and are puffed up in the newspapers, and private circulars, as infallible cures, in most all diseases which can be named, for either man or beast, for external appiication, or internal administration. No subject in medicine has been more fully exposed than the great and absurd pretensions of those medicines; but notwithstanding all this, the credulity of even the best class of society is great; finding their readiest victims among them. It surely requires no argument to show how dangerous must be the indiscriminate use of powerful drugs, compounded by parties who likely never had the slightest knowledge of a medical education, and how such persons can be able to cure, by their remedies, diseases which are, or may be, deemed incurable, and have defied the most consummate skill and experience of the veterinary medical world. 
Quinsy.-A name given to sore throat. (See Distemper and Influenza.)

Quittor.-This term is used in England for a disease in the foot of a fistulous character. (See Foot Diseases.)

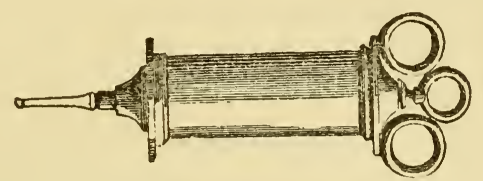

"Quittor Syringe, with Rings. To be Used with one Hand."

Rabies.-(See Hydrophobia.)

Rachitis.-A disease of the bones of young animals, and is due to a deficiency of earthy matter, (lime,) which causes the bones to yield, being too soft. In colts of the first year, some cases will be observed to stand so close at the knees, that one joint touches the other, which give the fore legs a curious looking twist, with the feet turned out, and the knees bent in. Colts so affected sonn get well when they are supplied with good, nutritious food, in which the phosphate of lime predominates. Rachitis, (pronounced racketis,) in old horses, is seldom seen; and when it is, it is in the bones of the back or lumber vertebræ, and is characterized by swellings of an irregular kind, with water oozing from them, and is called hydrorachitis or spina-bifida. Horses so affected, are not fit for work with weight upon the back. (See Deformities.)

Resolution-Is the most favorable termination of inor condition in which it was before it was attacked.

Respiration.-The alternate inspiration and expiration of air, performed for the purpose of exchanging the 
hydrogen and carbon for oxygen. The air being brought in contact with the blood, as it circulates through the lungs, the oxygen unites with it, and the nitrogen and carbonic acid gas is returned by expiration. The oxygen and some of the inhaled air is united in the lungs with free hydrogen, which is given out from the lungs, and is readily seen issuing from the nostrils on a frosty morning, or when the thermometer is about $40^{\circ}$.

Revulsion.-A second attack of disease, but in a remote or different part of the body from which the first attack had its seat. Example:-if an eruption, or the abscess of strangles be repelled from the outside of the body, we will find it attacking an internal organ. (See Metastasis.)

Rheumatism.-In no disease of the horse are there so many errors and mistakes committed; not only as to the nature of the affection, but its mode of treatment. Horsemen and horse doctors have not yet learned that there is a difference, and how to distinguish rheumatism from FOUNDER, whether acute or chronic. The difference between acute or inflammatory rheumatism, and acute founder, is this:-in rheumatism there is not only pain, but great fever and excitement; and its seat is in the joints of the legs; in founder, we have pain, but no fever; and the disease is confined to the feet alone. (See Lamenitis.)

In chronic rheumatism there may be some excuse for such mistakes, as there is no fever; but there is an inability to move, as if the horse was sprained over the loins. (See Lumbago.)

(1.)-Acute Rheumatism-Is nothing else but what is called (when man is the subject,) rheumatic fever.

Symptoms.-Great fever, excitement, and irritation, 
with extreme pain in the legs and joints; so much so, that the stricken horse has not a leg to stand upon, and dares not move from the place he occupies, for fear he would fall to the ground. In connection with all this disturbance, the horse sweats profusely, and blows or breathes excitedly; no heat in the feet, as in founder. It will be well to remember this, in forming a correct opinion of the case. In severe cases, the whole of the muscles of the body are set to quivering, clearly indicating inflammatory rheumatism in full force and degree.

Causes.-Sudden check to perspiration, by placing heated horses in a current or draught of cold air, thus preventing the transudation through the skin of its formed and natural secretion, and is absorbed into the blood, and acts as a poison, which produces inflammation in the sheaths of the tendons, and of the fibrous parts or tissue.

Treatment.-The treatment of acute rheumatism is sometimes unsatisfactory; not that it is incurable, but from the fact that the medicines which cure one will not do so in other cases.

Give 25 drops of the tincture of aconite root. every four hours, till six doses are given. Place the horse in a cool, airy place, with plenty of bedding under him, so as to induce him to lie down, and lightly cover the body, and apply cold water swabs or loose cloths to the legs, and keep them continually wet from twelve to twentyfour hours. In winter, warm water will answer best. By the time the six doses of aconite have been taken, a great change for the better will have taken place; so much so, that, in many cases, the horse may be left to nature to complete the cure. But, on the other hand, should the disease take a chronic form, give drachm doses of the powdered meadow saffron seeds twice in the day, 
and occasional doses of 60 drops of sulphuric acid in half a bucket of cold water. Half ounce doses of the sulphite of soda may be given as an alkali. Do not bleed or purge.

(2.) Chronic Rheumatism.-I have no hesitation in saying that chronic rheumatism in a joint is one of the most prolific causes of occult or hidden lameness in horses; and not only that, if there be no swellings to point to as a proof of the correctness of your opinion, many will pit upon your judgment; but it is with this, as with the first fruits of the earth-under a covering of snow; time is allowed for full development.

Treatment of Chronic Rheumatism. Give a few doses of aconite root, followed by the colchicum or saffron seeds-the sulphuric acid and the alkaline, as is recommended in acute rheumatism, but not pushing them to such an active extent. A liniment may be tried, applicd to the rheumatic joint or joints. Chloroform and olive oil, equal parts, to be used once a day, with friction by the hand. One part of the tincture of aconite root, may, in addition, be used to advantage with the chloroform.

(3.)-Rheumatism.-Accompanying Diseases of the Throat.-This form of the affection is often seen in such company, and is due to the fact that the same serous or fibrous tissue or membrane, is affected in each of these diseases; nevertheless, we do not see cases of throat disease following rheumatism; as by this, it seems that those membranes are not capable of reflecting upwards and backwards their sympathy or feeling, as the nerves of the body are. So, therefore, I am inclined to the belief that rheumatism in this form, is the effect of. disease in the throat, and not a cause of the disease. 
Treatment.-Cure the disease in the throat, and the rheumatism will be deprived of its cause and support. (See Influenza and Gastritis Mucosa.)

Ring Bone.-This is a serious affection, and consists of a circle of bone thrown out from the underlying bone, and sometimes, in addition to this, the cartileges of the foot are converted into bone, and laid in the form of a circle; and hence its name, ringbone, is most common in the fore-legs of heavy, coarse-bred horses, with short and straight up pastern-joints. When it occurs in fine-bred horses, it is usually the hind leg which is affected. Ringbone does not always cause lameness.

Cause.-Hereditary predisposition, from a peculiar formation of pastern-joints, which are found not well adapted to stand hard work, and hence, an effort of nature is set up to strengthen parts which are too weak, by converting an elastic substance into a hard and unyielding mass, and a moving hinge into a fixture.

Treatment. - If it is of recent origin, and the horse is young, much may be done in the way of a cure, by first removing all heat and inflammation with cold water cloths wrapped round the parts for three days, taking them off at night. At the end of that time, get one drachm of the bin-iodide of mercury, and mix with one ounce of lard, and apply one-half of the salve by rubbing it in well for ten minutes. Tie up the horse's head for a few hours, and the next day wash off with soap and warm water, and daily anoint the parts with lard or oil for a week; then apply the remainder of the salve in the same way, and proceed as before. In old horses, not much can be done with ring-bone, as the bones of old animals contain so much earthy (lime) matter that nothing can act upon it.

Ringworm.-(See Skin Diseases.) 
Roaring.-A noise made when the horse is put to work. There are many different sounds produced from the same cause, and are incurable, except they depend upon the presence of tumors, which can be removed. Roaring can be relieved somewhat by placing pads over the false nostril. When very bad, an operation called tracheotomy, or opening into the wind pipe, and keeping a silver tube inserted in it, is sometimes resorted to. By this means, a draught horse can be kept at work for many years.

\section{Round Bone.-(See Sprains.)}

Rowels.-An old-fashioned operation, consisting in an onening made through the skin for a few inches in length, and the skin is raised from its attachments, and a piece of leather fitting the cavity thus made is placed into it, so that a discharge is set up in a day or two. Times were when, and we too often see evidences yet of these cruclties unnecessarily inflicted upon the poor, unoffending horse. Rowels are an abomination, and inflict a scar or blemish, which never leaves the part. The stupidity and ignorance of horse doctors generally, do not allow them to see that the powers of nature are more potent for good in curing the affection which is attributed to the power or virtue of a rowel it never possessed. If I put in a rowel, it will not be with the view of curing disease or sprain, but for allowing the horse plenty of time in the stable, so that nature will have cured the disease herself. Gentlemen, give honor to whom honor is due!

Ruptures-Are the protrusion of some portion of the bowels or intestines out of their proper cavity and place. The groin, the navel, sides of the belly, and scrotum, or testicie bag, are the usual places where ruptures show 
themselves, and it is a variety of situation that constitutes the different species of rupture or hernia.

(1.) Inguinal Rupture.-In the United States, the male horses are mostly all castrated, which fact of itself accounts for the rare occurrence of this variety of rupture, for the operation of castration completely closes the inguinal ring or opening through which pass the spermatic cord, testes, etc., thus preventing the possibility of rupture in that direction. When this kind of rupture takes place, it is in stallions and uncastrated colts, and requires for its cure the castration of the horse or colt by what is called the covered operation, that is, by leaving the tunica vaginalis, or inner covering of the testes entire and uncut, and placing the clamps over it, and allowing the testes or stones to fall off, or be removed in two days from the time of operation. As soon as this variety of rupture is observed, have the horse or colt castrated at once.

(2.) Scrotal Rupture.-This variety of rupture is entirely confined to the testicle bag, or scrotal sack, and is also the affection of uncut horses, and is caused by relaxation of the fibrous tissue around the inguinal ring. This is a kind of rupture which comes and goes, as if it were in an intermittent affection. The rupture, or large swelling, during rest will entirely disappear, and return with exercise, and sometimes with violence; and throwing the horse into a fit of colic, will sometimes induce strangulation and death of the horsè. Scrotal rupture is sometimes confined to one side only of the scrotum. If in time the animal should not die from strangulation of the bowel, the rupture will sometimes increase to an enormous size, hanging far down, and filling up the space in and between the hind legs. 
Treatment.-The same as for inguinal hernia. Of course, in both cases, care should first be taken to push back the bowels through the ring into the belly, before removing the testicles. Scrotal rupture should not be confounded with hydrocele, or water in the scrotal sack. (See Dropsy.)

(3.) Congenital Rupture.-This is a species of rupture observed at the birth of the foal or colt, and is the least dangerous of all the varieties of ruptures, although the rupture continues to grow and increase in size until the fourth to the sixth month of the colt's age, and then gradually and progressively disappears altogether. If, however, it should not at the end of that time diminish in size and volume, a tolerably stout and tight collar or bandage may be placed around the body, and covering the rupture. This band should be kept in place by a broad collar or cloth attached to each side of the body bandage, and passing in front of the breast, and another round the back parts of the hips; thus preventing a backward or forward movement of the body bandage.

If rupture should occur in a few days after the birth of the colt, it should to all intents and purposes be classed as congenital rupture, and be treated accordingly.

Congenital rupture is the same as what is called by some writers umbilical rupture, which is correct as far as it goes; but congenital rupture includes not only the navel, but the scrotal also. The navel variety can be successfully treated by letting it alone, or in some cases the application of a bandage. And when in the scrotum castration is the only cure, a bandage in this case would not only be useless, but hurtful to the colt. 
(4.) Ventral Rupture.-This is when the bowel protrudes through any part of the belly, excepting at the umbilicus or navel, and not through any natural opening, and is generally the result of injury or accident, as from a hoov $\mathrm{k}$ from the horn of a cow, or the kick of a horse. The common place where this kind of rupture is usually seen, is on the lower portion of the belly, between the ribs and at the flanks.

Symptoms.-A large, puffy swelling; and by pressing against it, it will lessen in size, by its being forced into the cavity of the belly again; the skin will be loose when the bowel is thus pushed in. When the pressure has ceased, the enlargement or swelling returns at once, and fills up the loose skin.

Treatment.-In most cases, let the enlargement alone; as, in the majority of cases, no inconvenience from it will be experienced by the horse. The only way of reducing such a rupture is, by gathering and holding the loose skin, covering the rupture after the bowel has been pushed into its place, by means of long clamps, like a long vise, till the skin falls off. This cure is worse than the affection it is intended to remedy; for by breaking the skin the bowels are exposed to the air, and the uncertainty of the edges of the skin uniting firmly together. This is more than can be expected, as the horse is not a rational being, and cannot be told to stand this or that way, in this or that position; and if he experiences any pain, he will be restless, -lay down, and roll; and where are the nicely adjusted clamps?-The horse is dead in a few hours.

(5.) Rupture-Of Castration.-This variety of rupture sometimes follows immediately, or a few days after the operation of castration.

Causes.-When the rupture occurs as soon as the horse 
rises from the operation, it is produced by the violence of struggling, or rising with too much of a jerk. When occurring a few days after the operation, the cause may be laild to the wound not healing and uniting properly.

Symptoms.-As of colic; the horse rising, lying down, pawing, rolling, sweating, high fever and inflammation; and, finally, gangrene, or mortification of the parts, and death of the horse.

General Remarks. - The termination of ruptures of all kinds and varieties most to be dreaded, is that condition known as strangulation, which occurrence is indicated by the restive condition of the horse, pawing, rolling, sweating, etc., and if not relieved in a very short time, the horse will die. To reduce strangulation, the horse must be secured, and fastened; and every ingenuity must be tried, to get the bowels back into their proper place. No rule can be laid down to accomplish this, as some ruptures are reducable, and others are not. But the hands of the operator must be well oiled when handling the bowels, and the bowels kept scrupulously clean; and when they have been successfully placed into their proper cavity, the horse will be at rest, and relieved from pain. And to prevent the bowels from returning again, the rupture must be closed by skewers made of iron, or stiff wood, passed through the lips of each side of the wound, half an inch from the edge, and waxed cord wound round and over the skewers, in the form of the figure 8.

Metallic or silver wire is used by scientific veterinary surgeons in securing the edges of the skin of ruptures and injuries to the belly of all domestic animals, as the best and most successful plan.

Ruptures of the stomach, bowels and diaphragm, are 
occasionally the immediate cause of leath in cases of colic.

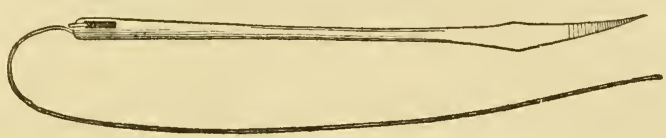

NEEDLE ARMED WITH WIRE.

Saddle Galls.-Sores produced by the saddle and other portions of the harness, and are best treated by the compound tincture of aloes or myrrh. When the sores become hard and firm, like warts, use the ointment of iodide of mercury. (See Medicines and Prescriptions.) Remove the cause of the trouble by attending to the saddle and harness, and if there be no fault to be found with the stuffing of the saddle, cut a hole sufficiently large in the padding to accommodate the sore without touching it.

\section{Sallenders.-(See Mallenders.)}

Saliva.-The fluid which is secreted by the salivary glands into the mouth, and is swallowed with the food. When horses are feeding, the saliva mixes with the feed, and resolves, dissolves, and changes it into a soft mass fit to be swallowed. (See Parotid Duct.)

Salivation.-This is an increased flow of saliva induced in the horse by mercury and other medicines, and is often seen in horses which are feeding upon the second crop of clover late in the fall of the year, or after slight frosts; which fact sufficiently accounts not only for the salivation of, but also the reason why, horses so feeding lose flesh and become thin and weak, although apparently feeding upon abundance.

Second crop clover grows fast, is soft, and full of moisture, and cool nights arrest its mush-room growth, and 
the moisture it contains is great in quantity, and is converted from a sweet and nutritious substance into a sour and acidulous fluid, not unlike vinegar or acetic acid. The acid so formed within the clover leaf and stem, is pressed out by the act of mastication or chewing stimulating the fauces of the mouth and the salivary glands to such an extent as to tax the substance of the body to supply saliva to meet an enormous demand. Hence, the weakness and loss of flesh of horses so fed.

Sand Cracks.-(See Foot Diseases.)

Sanious Pus.-Pus mixed with water and blood;unhealthy pus.

\section{Scalds.-(See Burns.)}

\section{Scald Mouth.-(See Mouth Diseases.)}

Scarlatina.-A disease newly discovered in lightcolored horses, attended by sore throat, as in man, and with slight fever and dry skin-the glands of the neck are slightly swollen, and in about two days the lining membrane within the nose and lips become studded with scarlet spots of the size of a garden pea, which run together. As before stated, in light bay horses will be seen patches from which the hair falls off, and is replaced by a coat of a lighter color, thus making the animal look as if he had had an attack of varioloid or small-pox. Scarlatina is an eruptive fever, running a fixed and definite course, and is closely allied to purpura, influenza and strangles, (all of which see.)

Treatment.-Place the horse in a cool place, and give small doses of aconite followed by gentian and carbonate of ammonia. In a few days iron may be added. (See Medicines.)

Scratches.-(See Grease and Phagadena.) 
Schirrus.-Pronounced skirrus, and signifying indu ration or hardening of any structure, but now used only when speaking of cancer, (which see.)

Scouring.-(See Diarrhœa.)

Scrotum. - The bag or skin covering the testicles of the stallion, and is the seat of large watery swellings, as a sequel to debilitating disease, or disease treated by starving, bleeding, etc. If the swelling does not grow less after extra feed is allowed, and a few iron powders are given, the scrotum will have to be opened a little on both sides to allow the fluid to flow out. Do not mistake the swelling for scrotal rupture, (which see.)

Scurf.-A scaly eruption on the skin of badlygroomed and cared for horses, and is cured by good grooming, good feeding, and, in some cases, a change in stabling.

Secretion is the product secreted or separated from the blood. Secreted products are of two kinds:

(1.) Excretions, or matter separted by animal bodies, and thrown off on account of their noxious or effete qualities. Examples-the urine, the dung, sweat, and carbonic acid gas from the lungs.

(2.) Secretion is matter separated from the blood for further use, and for the performance of various actions in the living system. Examples-bile, saliva, etc.

Sedatives.-Medicines which lessen pain, and should, therefore, be called calmatives. Aconite is the most certain and successful sedative and calmative we have to offer, and will seldom disappoint the highest expectations, if used properly, and in good time.

Serum.-The thin, colorless fluid, which separates from the blood. 
Serous Abscess.-A variety of abscess seen usually about the breast of horses, and arises from injury followed by the pouring into the injured part, a serum from the blood, and not being absorbed readily, it, therefore, remains there in the form of a large ovoid flattened bag, which a blind man, who never saw a tumor, can tell it contains fluid. Indeed, wherever dropsical swellings are not absorbed, a serous abscess will be formed.

Treatment.-Open it with a large knife, or with a small one, making a large opening through the skin, only at the lowest soft part, so the serum will run out without pressing. Inject once or twice with cold water, and anoint the skin to prevent it from cracking or becoming dry.

Seton.-A piece of tape placed under the skin by means of a needle, made for the purpose. Setons are rarely of use, and are often torn violently out, making an ugly sore.

Shivers.-So called because the horse is seized with tremor of the muscles of the whole body, when any attempt is made to push the horse back. The tail is erect as in cases of locked-jaw.

Causes.-Tumors on the ventricles of the brain.

Shoeing of Sound. Feet.-Most diseases of the feet, and every stumble is, either directly or indirectly, the result of bad shoeing. IIorse-shoers have long been in the habit of using the knife and rasp too freely to keep feet sound very long so. When the shoe is carefully removed, the wall or crust, where a well seated shoe should only rest, should be gently rasped to remove fragments of loose horn and old nails. In deep, wellmade feet, the sole requires paring out till it is in the 
form of a cup, but not too thin, the bearing surface, or wall, to be made level for the new shoe. This is all that is required in a sound foot. The frog must be left to fill its functions. Above all do not let the rasp be used upon the surface of the foot, for it is the skin of the hoof, and by its removal you expose it to every change in moisture and dryness, and leaving it weak, drey, porous and brittle.

The shoe should be a plain one, and equally broad and wide from heel to toe, and put on without seating; for why bring a concave foot in contact with a concave shoe? The toe should be slightly turned up, and not too short at the heels. The hind shoes should be provided with heels.

The nail holes should be three on the outside, and two on the inside, and made straight through the iron, and not incline inwards, and the shoe fitted to the foot, and not the foot to the shoe.

Dray horses should be shod with tips, or toes and heels which secure firmness of tread, and greater power when drawing heavy loads, and especially in cities with smooth paved streets.

Shoeing Unsouxd Feet.-Feet with corns, weak, flat feet, convexed sole, and sand or quarter cracked feet should have shoes well-seated, and is advisable to throw some extra weight upon the frog, and for this purposea barshoe should be used. (See Foot Diseases.) Leather soles are useful in weak-soled feet, and when the horse steps high, and is much used upon city streets. One sided nailing answers well for weak heels. Ring Boned animals should be shod with easy fitting shoes, to aroid jarring. Horses having, or have a tendency to navicular or coffin-joint disease, should have shoes turned up a 
little at the toe, with the ground surface of the wall well cut away, and the sole and frog untouched. The art of shoeing horses consists in fitting a shoe to the foot of a horse, for the purpose of protecting, at the same time not to injure it.

Shoulder Lameness.-Is produced by a slip or sidefall, and is frequent with horses in cities, with broad rails laid on the streets for railroad purposes, and in wet weather these rails are very slippy; hence the horse has no foot-hold, and the leg is stretched far out before the animal, and the muscles of the shoulders, and in some cases, the shoulder joint is involved, a much more serious affair than simple shoulder sprain is the result.

Shoulder Lameness is common to young horses when ploughing in the furrow.

Symptoms.-The absence of heat, tenderness, and swelling in any other part of the leg or foot. Always find out with certainty, where there is no lameness, and the situation of lameness will very soon be apparent. Negative and positive symptoms should always be well considered before coming to a final conclusion, as by doing so the situation or trouble is so narrowed down that a mistake can scarcely occur. Shoulder lameness, however, is known by the horse stepping longer with the lame leg, and shorter with the sound one, and excepting in very severe cases the horse will not only point the leg out from the body, but carries it also to the side of the body. Now in most sprains and diseases in the foot, the leg will be pointed straight out without any side position. Take the leg which is lame by the pastern, and gently carry, or pull it straight out from the body of the horse in front, and gently also to the outside, and if it be shoulder lameness, the horse will not only show 
evidences of pain, but will in many cases, depending upon the spirit and animation of the horse, get up from the ground with the sound leg and endeavors to wrest the lame leg from you.

In very severe cases, when occurring from a bruise, the horse will stand on his toe, which is evidence of contusion of the shoulder.

Treatment.-Absolute and entire rest, warm water cloths applied for two days, and followed by cold water ones, in the same way, for as many days. Then a slight blister of the Spanish fly may be rubbed into the skin of the shoulder, taking care that none of it is put on the situation of the colar, as it would render the part a little tender for a while by friction from the colar. Spanish fly in powder, one drachm; hogs lard, six drachms. Mix, and make an ointment, or salve, and rub in the better half of it into the skin; next day wash off with warm water, (not hot) and when dry from washing, annoint the blistered parts with oil or lard, daily for a week. It is not advisable to put horses to work, or even exercise, too soon after getting well from lameness.

Shoulder Joint Lameness.-This is a more serious form of lameness, than sprain of the muscles of the shoulder, and consists in softening of the articular cartilages of the joint, with inflammation of the joint membranes, and great secretion of unhealthy synovia, (joint oil) which produces bulging of the ligaments, (capsular) covering the joint, and can be detected by making the horse stand upon the lame limb, by holding up the other one. This is a disease similar to spavin in the hock-joint, which accounts for the unsatisfactory result of treatment.

Symptoms. - The horse drags his toe, and throws his leg out at every movement of the limb. 
Treatment.-As before stated, this is rather unsatisfactory, from the fact that the cartilages are likely to be destroyed, and the bone underneath apt to become ulcerated, but if taken in time, much good can be done. In many cases, a cure can be effected by the ointment of red iodide of mercury, well rubbed in once a week, for a few times.

Take of biniodide of mercury, two drachms; hogs lard, two ounces. Mix well on the bottom of a dinner plate, or a smooth slate with a table knife. And of this ointment, take one fourth and rub well into the joint, and tie up the horse's head for a few hours, to prevent the horse from getting at the shoulder with his mouth, and allow soft bedding for the front feet to stand upon, as the horse will stamp with his foot on the floor, for the action of this ointment is considered to be as painful as the hot iron, for half an hour from the time it begins to aet, till the parts commence to swell from its splendid effects. Hence, firing irons are now not much used, except in the hands of old fogies. Daily, oil or grease the parts for a week, then apply as before, and remembering that to get all the benefit of this ointment, it must be well rubbed in to the parts.

Side Bones-Are a species of ring bone; only the side cartileges of the foot are converted into bone, and does not, as in ring bone, extend round the coronet, or portion immediately above the hoof. The cause and treatment are the same as that of ring-bone (which see.)

Sinus.-A long, narrow and ulcerated track, communicating either with the inside of an abscess, or diseased bone .(See Fistula.-Poll Evil.)

Sitfasts.-These are hard and insensible tumors, 
and sometimes called warbles, caused by undue pressure from the harness.

Treatment.-Rub in, about the size of a bean, of the ointment of red iodide of mercury. (See Prescriptions and Medicines.)

Slrin Diseases.-The many, and apparently different varieties of skin diseases described by writers, many of which are the same, and produced by the same cause, but present different appearances in different animals, and in different stages and conditions of the affection; and where the same cause can be properly assigned as producing different diseases, although apparently dissimilar, the treatment must be the same. Thus: if the acari is the cause of more than one kind of skin disease, of course the treatment must be directed to the destruction or removal of this insect or mite, before a cure can be effected; so, also, with faulty assimulation or digestion, which so often give rise to skin disease, will have to be improved and corrected before the effect, (disease,) will cease and be cured.

(1.) Baldness.-Parts of the skin of the horse become denuded of the hair, and occurs from minute or small pimples which contain a fluid, and burst, or break, carrying the hair with it. These pimples, or small tumors, however, are sometimes vesicular, sometimes papular, and sometimes scaly, and is caused by faulty digestion, and is treated by soft feed, or fresh-cut grass. The hair will grow again.

Baldness is caused by scalds, burns, and blisters; and where the true skin is not entirely destroyed, the hair can be restored by using a weak ointment of iodine-iodine half a drachm, hog's lard, eight drachms, mix, and apply with rubbing with the hand, once every third day, till 
there are evidences of a growth of hair springing up. Gunpowder and lard have no more power in causing hair to grow, than as much lard, saltpeter, sulphur and charcoal would have, and is not to be compared to the iodine, because, if iodine does not restore the hair in all cases, it will certainly dye or stain the skin a dark color, which cannot be washed off; and hence, in dark-skined horses, is of much use in taking the bare, bald-look off a white spot, on a dark-skinned horse.

(2.) Mange, Itch, Psora, or Scabies.

Cause.-The result of an insect breeding and burrowing in the skin, and is called acari, a variety of mite or animalculæ.

Symptoms.-At first, a fine crop of pustules, not at this time always seen, about the head and neck, and under the mane. By the horse rubbing himself against whatever he can get at, the hair falls off, and exposes an angry and red-colored skin, with red points and lines, fissures, wrinkles, or scratches. After this condition, we have dryness, scruffiness, baldness, and whitening of the skin, accompanied with great itchiness.

Treatment.-The best plan for curing this disease in horses, is as follows: Take fine sea-sand, such as is used by stable-men for scouring steel bits, and rub the affected parts well for a few minutes. Then wash the parts well with good soap and water, and a brush, after which dry the parts wehl. Then anoint the parts with the following ointment: Powdered sulphur, one ounce, hog's lard two ounces; mix. The following is more cleanly: Take liver of sulphur, or hepar of sulphur, two to three ounces, cold water one quart; mix, and make a wash. This plan, and either of these mixtures properly applied, will not only kill the insect, but will cure a disease which has baffled 
many who have attempted its cure, without first exposing the insect, by scouring him out of his covering, and killing him with sulphur, which is so objectionable to parasitic life.

Observe.-This disease is contagious. Stall-posts, mangers; harness, combs, brushes, etc., used about the horse, should be subjected to heat equal to boiling, either by boiling or steaming. Trees, gates, and rubbing-posts in the field, should be washed with water, and coated with a mixture of sulphur, lime and water.

(3.) Eczema.-This is an affection of the summer months; and by close inspection, large numbers of minute elevatiuns, or raised parts, closely joining each other, and filled with a watery fluid, will be observed; the skin will soon present a red and angry look, the hair short and dry, accompanied with extreme itchiness; so much so, sometimes, that horses so affecter become almost unmanageable. The situation of this disease is usually in the hind legs, and is considered hereditary. At all events, when a horse is once attacked by it, it is liable to return again with the warm weather.

Cause.-A peculiar condition of the blood, developed by heat.

Treatment.-This disease in my hands, in several carriage horses which were rendered completely useless in the summer months, have not only been cured, but it has been prevented from returning, by administering half ounce doses of the sulphite of soda, for two weeks previous to the hot weather, once a day; and in addition, a few bundles of fresh grass, cut from gentlemen's garden borders, were given. The soda, to neutralize, ferments in the blood, and the grass, fresh cut, to assist, by its 
action on the stomach and bowels, and ultimately upon the blood itself.

(4.) Surfeit.-A disease in the spring of the year in fat horses, short and well built, and is characterized by tumors about the size of a grain of Indian corn, covering the whole borly as completely as the nettle-rash does that of children, and does not generally cause itching, nor yet, like some skin diseases, is it contagious.

Cause.-Robust stamina.

Treatment.-Give soft or slop feed composed of bran, cut hay, cold water, and salt for a few days. Give grass, if it can be had, without any additional feed. A few doses of the sulphite of soda will be of advantage.

Bleeding and purging I have seen arrest the tumors in their growth, but unfortunately they become permanent fixtures, only to be removed by calomel and opium, to cause their absorption. Calomel, thirty grains; opium, ten grains; mix, and give once a day, for a week or ten days. Good feeding will here have to be given.

Rixgworm, Porrigo, and Favus, are names given to this disease.

Symptoms.-Small circular patches, or scales, sometimes running together, forming large crusts and ulcers under them, in which insects breed. This is a contagious disease, and is communicated from man to animals, and animals to man. The microscope has shown the presence of fungi in the scab of ringworm, but whether it be merely the effect of the disease, is not at present known. It is, however, more likely to be the cause.

Treatment.-Wash and keep the parts clean, and aim at destroying the fungi; and for this purpose remove the scab as soon as formed, and apply a solution of oxalic 
acid to the surface, fifteen grains of the acid to an ounce of water. Tar ointment, creasote, sulphur, and mercury, have all been tried; but none of these meet with so much success, as the solution of oxalic acid just recommended. For other diseases of the skin, see Grease, Mallenders, Sallenders, Tumors, and Boils.

Slobbering.-This is produced in horses by many causes. Large doses of aconite, veratrum, sore, or scald mouth, and in some cases of poisoning. Slobbering from eating second crop clover, will be found treated of under the article Salivation, (which see.)

Slough-Is the separation of a diseased, or dead part from the healthy portion. A slough may be of more or less thickness, and may include the skin and flesh to a considerable depth, as from the centre of an abscess. (See Gangrene and Mortification.)

Sores.-Healthy and unhealthy sores occur in, or on, all parts of the body of the horse. Healthy sores are best treated by the tincture of aloes, or myrrh, or simple ointment. (See Medicines and Prescriptions.) Un healthy sores should be treated, first, by the application of some caustic, or powdered blue stone, nitrate of silver, or caustic potassa, which will make an unhealthy sore a simple and healthy one, to be treated as the above.

Sore Mouth.-(See Mouth Diseases.)

Sore Foot.-(See Foot Diseases.)

Sore Throat.-(See Catarrh, Cold, and Bronchitis.)

Sore Back.-Re-stuff the saddle, and apply the tincture of aloes or myrrh.

Sore Shoulders.-See to the collar, and apply as to the above.

Sore Neck.-There is a variety of sore neck on the 
place where the collar rests and presses, when descending a hill in double team, which is very troublesome, and difficult to heal, if the horse be kept at work, and causes the animal great irritation and uneasiness; and when the hand is laid upon it, the horse, if he be a spirited animal, will plunge in the stall, and even kick, however quiet he may be at other times.

Treatment.-Apply simple ointment, and place a firm, but strong piece of leather over it, before the collar is put on, for it to rest and slide over, instead of upon the mane and neck.

Soundness - Is when a horse has nothing about him, that does or is likely to interfere with his feeding, working, and general usefulness.

Spavin-Is a variety of disease affecting the hockjoint; but spavin is not now looked upon as in the days of Oliver Goldsmith and William Shakspeare. Because in the minds of those distinguished men, and of some of their readers of the present time, spavin is an enormous enlargement of the hock of the horse; whereas, in some of the worse forms of spavin, there is no enlargement at all; and yet, the hock joint is completely destroyed, stiff, or anchylosed. Shakspeare thus refers to Petruchio's horse:- "His horse hipped with an old mothy saddle, the stirrups of no kindred: besides possessed with the glanders, and, like to mose in the chine, troubled with the lampas, infected with the fashions, full of wind galls, sped with spavins, raied with the yellows, past cure of the fives, stark spoiled with the staggers, begnawn with the bots, swayed in the back, and shoulder shotten, ne'er legged before, and with a half-cheeked bit, and a head stall of sheep's leather." 
(1.) Bog Spavin.-This kind of spavin is situated in front of the hock-joint, and is a soft, fluctuating swelling, which rarely ever causes lameness. It is merely an enlargement or distention of the bursal cavity of the joint, and is filled with the natural fluid of the joint, but increased in quantity, and possibly, in some cases, a little changed in quality also.

(2.) Blood Spavin.-Is the same as bog spavin, but more extensive, and generally involving the hock-joint on its three sides, front, inside, and outside, and giving to the limb a thick,rounded appearance, called thoroughpin, (which see.) The swelling is soft and fluctuating, and indeed there is no perceptible difference in the nature and result of this form of spavin, and the above variety, only being more extensive, interfering, perhaps, with the flow of blood in the sub-cutaneous, or vein under the skin, which is seen in front, and partly to the side of the joint, adding very little to the general swelling.

Causes.-Hard work and fast driving, especially when horses are young.

Treatment.-The application of the ointment of red iodide of mercury, in the hands of some, has done much good; but the enlargement is apt to return when the horse is again put to work, from the fact that the more joint-oil, or fluid is poured out, and hence the cause of its return. Acupuncturation, or forcing small steel instruments into the swelling to let out the fluid, after which a bandage or truss is fitted to the part to press out the fluid that remains, and to cause adhesion of the parts together are sometimes resorted to. The difficulty in curing soft spavin, is the danger of opening into a cavity or joint containing oil, (synovia.) An old way of treating such 
disease, was by striking the parts with a mallet, so as to break the skin, so that the fluid would eseape; and the blow given to the skin being so great as to set up adhesive inflammation, and closure of the opening.

(3.) Bone Spavin.-Consists in the sprouting out of irregular boney matter from the bones of the joint, preventing their smooth and proper action over one another, and causing lameness. This bony growth sometimes grows to a good size, and in some cases we find one or two of the bones only involved; at others nearly all of them are affected. The situation of bone spavin is on the inside, and in front of the joint.

Symptoms. - When horses are what is called breeding bone spavin, ordinary persons think and say the lameness is in in the hip, (see Hock) from the action which is reflected by the muscles of the hip at every movement made by the hock-joint, and from the fact that there is no enlargement as yet in the joint. The horse is lame on starting, but gets better after a little while. After standing will start lame again. In the stable, he shifts one hind leg after another, and when resting the lame leg, he stands on the toe. Pain and lameness cease when the joint is consolidated, although remaining a little stiff.

(4.) Occult Spavin.-This is a disease similar to bone spavin, the difference being no enlargement of the joint whatever, although the bones of the joint are all diseased, immovable and stiff. This disease seems to puzzle and perplex horsemen, because they cannot comprehend a bone spavin without an enlargement of the joint outside; and, consequently, the poor horse is sometimes blistered and tormented in every part of the leg, but the right place. The cause, résults, and effects of 
this disease are the same as in bone spavin, excepting there is no enlargement.

Treatment.-For the two varieties of spavin, just described, the treatment will be the same. In young horses, the red iodide of mercury in ointment, is the proper treatment. One drachm of biniodide of mercury and one ounce of lard. Mix, and apply once in a week, and lard the parts once a day, till the next application.

Old horses should have a liniment applied, once every second day, to the parts. Oil of olives, two ounces; oil of turpentine, one ounce; creosote, one ounce. Mix. This will relieve the pain, and to a great degree the lameness; also, spavined horses should be allowed an extra allowance of feed, to keep them in condition equal to their more healthy and fortunate neighbors.

\section{Specks on the Eye.-(See Eye Diseases.)}

Speedy Cut.-Is an injury to the knee from the shoe of the opposite fore-foot, and is prevented by driving slower, and nailing the shoe on one side only.

Treatment.-The application of cold water cloths, and if the swelling does not all go away, follow with the application of the red iodide of mercury, in the form of an ointment, once a week for a few times, and lard or grease the parts till the next application. Boots are sometimes used as a preventative.

Spleen Diseases.-The diseases of this organ are obscure, and chiefly consist of enlargements, tubercles, softening, rupture, and tumors.. The symptoms attending these affections are not well marked.

Splint.-A small bony enlargement, situated usually on the inside of the fore-legs, about midway between the knee and the pastern joint. When splint begins to 
grow, they sometimes produce lameness by stretching the covering of the bone, which however soon accommodates itself to the altered structure.

Cause.-Working horses when too young, and before the leg and splint bone have become united. Hence, splints disappear when the horse grows older, and the unity of these bones take place.

Treatment.-One or two applications of the ointment of red iodide of mereury, or tincture of Spanish fly, one ounce; oil of eroton, twenty drops. Mix, and apply with rubbing.

Sprains.-By this term is meant partial displacement, twisting of a joint with more or less injury to the articulations, ligaments, tendons and their sheaths, and even small portions of the bones of the joint are separated. Indeed, every variety and degree of severity is to be seen in sprains of different parts. Every joint is liable to sprain, but the usual or more common sprains will be found in the pastern or fetlock joint, shoulder and its joint, hock, stifle, back and loins, flexor tendon, suspensory ligaments, etc., produced by a common cause, slipping, falling, and over work, and the weight of the body, and not unfrequently the load falling upon a part, when not in its proper position for receiving it.

Symptoms.-Pain, heat, swelling, tenderness and lameness, more or less severe, depending on the severity of the sprain, and the part affected. In severe sprains, care must be taken to guard against mistakes, and to form a correct judgment of the nature of the injury, whether it be a real or simple sprain, or whether there be fracture or dislocation.

I'reatment.-Absolute and entire rest, is the principa point to be attended to in the cure of sprains, for no 
sprain cannot be cured without rest, no matter what other remedies are employed; and, if there be much fever and excitement about the horse, a few doses of aconite will have to be given. Tincture of aconite root, fifteen drops, three times in the day for two days, will remove fever and irritation.

Then apply warm water cloths for three days, followed by cold water cloths for the same length of time, taking the cloths off at night, as it is necessary that water must be poured on the cloths every hour, or before the warm ones become cold, and the cold ones become warm. After which time the lameness and swelling will have ceased, or whether this be not the case, apply for a few days, once a day, the following liniment : creosote, one ounce; oil of turpentine, one ounce; oil of olives, two ounces; mix. Be assured the horse is quite well before he is put to work again, as many joint-diseases are brought on by mis-managed sprains, which never can be cured. For sprains of different parts of the body,-(See Shoulder and Shoulder Joint Sprains, etc.)

Staggers.-A disease familiar to every horseman, a serious disease, and presenting different symptoms in different horses, depending to what extent the brain and nervous centres are affected, and whether the variety be mad, grass, stomach, or sleepy staggers.

(1.) Stomach Staggers.-This is an attack of acute inaigestion, from overloading the stomach; digestion is arrested, fermentation is set up, and the evolution or giving off of carbonic acids gas distending the stomach and bowels, and pressing on the space alloted for the lungs to play in, depriving them from æriating the blood, thereby affecting the healthy action of the brain and 
nervous cenres, and consequent death of the horse in from twelve to twenty hours.

Treatment.-Injections of warm water and soap, and a handful of salt to clean out the bowels, so the gas can get free passage. Arrest fermentation by dissolving two ounces of the sulphite of soda in a little water, to be given at one dose, the dose to be repeated every hour. Eight drachms of powdered aloes should be given, mixed in a little warm water, and drench the horse with it to stimulate digestion, and open the bowels.

(2.) Grass or Sleepy Staggers.-Is a chronic variety of stomach staggers, and should be treated as for the above variety.

(3.) Mad Staggers.-This is inflammation of the brain, and is sometimes called Phrenitis.

Symptoms.-Dulness, followed by excitement and madness. The sleepy stage, or the congestive period passing off, then the madness is seen, and the horse unconsciously throws and dashes himself about, and some times endeavvors to climb up the wall, and at other times, ropes will have to be used to keeph im from pulling back, and becoming unmanageable, and destroy other horses, harness, carriages, and even the stable itself. Such is the power of a mad horse,-a painful sight to see. Finally he becomes exhausted, falls and dies,-a great relief indeed.

Treatment.-The horse is not worth saving, and rarely can be saved; for nothing can, or will, give relief to a mad horse, but bleeding, and this to so great an extent that life does not rally; and the horse dies, a dull, stupid, and immovable mass, which can neither eat, drink, nor digest. The brain is pressed with fluid and lymph, between the pia matter and the archnoid. 
Remove a mad horse, as soon as observed, from any thing of value, that can be broken or hurt, or remove the things, or other horses, from the mad one, before it is too late.

Staked.-An accident to some portion of the body, but most frequently to the belly, by horses leaping fences, or it may be by the horn of an ox, cow, or bull.

Treatment.-If the injury be on the belly, and the wound deep, and have entered the belly, ascertain, with the finger, whether any portion of the bowels are injured, or if any portion of the bowels has escaped through the opening; and if so, and part of them be torn, sew with small, fine cat-gut, (which is used by fishers with the rod, and with artificial flies, ) and pass the bowel or intestine into its proper place, and close the wound in the same way as is recommended for rupture of the belly, (which see.) If the skin is only wounded, treat it as for simple sore. If the wound is in a fleshy part, and the skin peeled or torn from the flesh, it had better be clipped off, as it will not unite again, but shrink and dry up; hence, it is not advisable to let it hang, nor attempt to sew it. Trim off the fragments of loose skin, and treat the wound with a weak solution of blue stone, chloride, or sulphate of zinc. (See Medicines and Prescriptions, and Bleeding Wounds.)

Stings from Bees, Hornets, etc.-Not unfrequently we hear of horses losing their lives from irritation and fever, set up from the effects of stings from those insects.

Treatment.-Take acetic acid No. 8, four ounces, powdered camphor, one ounce; mix, and dissolve, then rub a portion of this mixture into the parts most affected. The poison, swelling, irritation, etc., will be at once arrested. In an hour afterwards, anoint the parts with 
sweet oil or lard. As acetic acid may not be at hand in an emergency like this, strong table, or white wine vinegar should be used, without the camphor. Acetic acid of the French, is eight times stronger than ordinary vinegar.

Stifle Joint Lameness.-In the article Hock-Joint, I have stated that this form or situation of lameness in the horse, is not so common as horsemen and others suppose it to be; nevertheless, no part or function can be declared exempt from accident and disease. It is chiefly, however, an affection of voung colts, on uneven ground, and arises from wearing away the toe of the foot, thus inducing dislocation of the stifle, or patella, which slides off the rounded heads of the bones, at every step the animal takes. (See Patella.)

Treatment.-Remove the horse to level pasture, and have him shod with a shoe, having a projecting piece of iron attached to the toe of the shoe, which will prevent the bones from sliding out of place, and knuckling at every step.

Stifle Ulceration.-This is a disease of the halfmoon shaped cartilages of the joint, and a serious disease, as all joint affections commonly are.

Symptoms. - The leg is held firmly, and forward of the other, and is thrown outwards at every step. The joint is enlarged, and the capsular ligament very much distended, especially when the animal's weight is thrown upon it, by holding up the sound leg.

Treatment.-Not satisfactory; as ulceration is generally progressive in its character, and frequently destroys the heads of the bones. But the ointment of the red iodide of mercury may be tried.

Bin-iodide of mercury, one drachm; hog's lard, one 
ounce; mix, and apply with rubbing with the hand, once a week, for a month. Lard or oil the parts daily, between each application.

Stomach Diseases.-(See Gastritis Mucosa, Staggers, Colic and Enteritis.)

Stone in the Bladder.-Are those concretions named calculi, formed in the bladder from a diseased condition of the urine.

Symptoms.-Pain, occasional stoppage in the stream of the urine before all is passed, a straddling gait.

Treatment.-Is the province of surgery.

Strains.-(See Sprains.)

Strangles.-This is an eruptive fever, characterized by swelling in and between the bones of the lower jaw, terminating in an abscess.

Cause.-A specific poison in the blood, which few, if any horses which live ten years, ever escape. It especially attacks young horses, but it is often seen in horses of increased years. Sometimes, the enlargement or abscess of the strangles, is seen on the side of the face, and even on other portions of the body.

Treatment.-Give the horse grass, or soft feed, and little or no medicine. The appetite will return when the abscess is opened, or breaks of itself. This is the great secret in the treatment of strangles. Do not poultice the swelling, as it will only thickens the skin. If any thing is to be done to hasten the abscess, rub in a little of the ointment of Spanish fly. (See Medicines and Prescriptions.)

Stranguary.-This is a name given to the urine, when mixed with blood, and when pain is present when the horse is urinating. 
Cause.-Irritation of the bladder or kidneys, or both, from the use of the Spanish fly, either in the form of a blister, being too extensive, or too near the loins and kidneys, and from the fly being given in an improper manner, and in too large doses.

Symptoms.-Frequent desire to pass urine, and when passed, it is in small quantities, and bloody. Pain, irritation, and fever, are present.

Treatment.-To remove the pain, give twenty drops of the tincture of aconite root every three hours, and give plenty of cold water to drink; also flax-seed tea, to horne, or drench down the throat of the horse, to sheath the parts from irritating substances. Equal parts of good mustard and flour, mixed with warm water, and made into a soft paste, and laid over the region of the kidneys, or small of the back, and occasionally moisten it with warm water, and cover it with a dry cotton or linen cloth.

Strangulation.-This is a term applied to a part which is tightened, contracted, or closed. Strangulation and stricture are terms implying different degrees of the same phenomena. Thus we speak of strangulation of the bowels from rupture, and of the glottis or head of the wind-pipe, when, from some cause or other, it is closed. Strangulation and death of the horse is the result, if no opening be made lower down in the wind-pipe, to admit atmospheric air. Stricture is that condition of the windpipe or glottis, when contracted or pressed upon by enlargement of the glands of the neck, or thickening of its own membranes, diminishing the space or width of its caliber, giving rise to thick wind, and increased breathing and cough.

Stringhalt.-This is an affection of the hind leg, and 
is known by the peculiar way the hind leg or legs are raised from the ground-a quick, spasmodic jerk.

Causes-Are two fold. First. The loss of nervous influence, whereby the extensor pedis muscle, and possibly some others, are deprived of their proper power. Second. The peculiar anatomical articulation, and general structure of the hock-joint of the horse are such, that when the leg of a dead horse is stripped of its muscles, the ligaments not disturbed at all; and if the legs above and below the hock be caught hold of by the hands, and the leg straightened out, the moment the hands are taken from it, it will spring into a bent position on the instant, thus imitating stringhalt, as near as can be. Thus the balance of power is not equal; the articulary ligaments of the hock are stronger than the muscles of the thigh. Hence, the moment the horse lifts his foot from the ground, the leg is instantaneously snatched up by the power of the articulating ligaments.

Treatment.-Restore the lost nervous influence; thereby the muscles of the thigh are to be brought into healthy action. This will best be done by good feeding, and one grain of strychnia nux vomica given daily, for six weeks in the horse's feed.

Suppuration.-This signifies the secretion of pus, and is one of the terminations of inflammation.

Symptoms.-Whenever suppuration is going on to any extent in or on the body of the horse, a shivering fit, similar to a chill, will or may be seen, followed in a day or so, either by a discharge of a yellow pus from the nose, or the formation of an abscess on some portion of the body. Suspect suppuration when rigors and shivering occur, especially after accidents of whatever kind.

Treatment.-First. Support the strength of the horse 
by good feeding, whereby the process will soon be over, and without any complication with it. Second. Do not physick or bleed, for those measures would prevent the process of suppuration from going on in its original place, and perhaps drive it to another. (See Metastasis.)

\section{Surfeit-(See Skin Diseases.)}

Swelled Legs.-(See Grease, Sprains, Debility, and Dropsy.)

Swellings. - Are of different kinds-the hard, inflammatory swelling caused by injury, suppuration, and diseased bone-the soft and fluctuating swelling, which is generally circumscribed, and seen in disease of the joints, as blood spavin, etc., and contain joint oil-the dropsical, or soft and non-inflammatory swelling of many and different parts of the body, at one and the same time. Each must be treated according to the cause of the swelling.(See Farcy.)

Sympathy.--Is that condition which is developed in one part of the system or body of an animal, although not of itself a disease, but is on account of disease or accident happening to some other, or remote portion of the body. This sympathy is communicated through and by the nervous systern. A nail in the foot, or a broken bone, is immediately followed by sympathetic fever, and general disturbance of the whole system. Herein lays the great difference between the animal and vegetable life. A branch of a bush or a tree can be lopped off without any disturbance to the life or health of the main trunk. No such immunity exists in the animal creation.

Synovia.-A clear, colorless and viscid fluid, secreted by the lining membrane of the joints, for the purpose of 
preventing friction, and allowing of easy motion of the heads of the bones over one another. Synovia sometimes changes in quality, becomes thinner, and gives rise to swellings of the joints. (See Blood and Bog Spavin.)

Synchronous.-This name is applied to the action cr beat of the heart, when it is in time with the beat or pulsation of an artery.

Systole.-The movement or contraction of the heart, as the systolic murmur.

Tabanidœ.-A species of fly which are a great annoyance to horses.

Tabes.-Wasting of the muscles of the body. (See Mesenterica.)

Talpæ.-A wart-like tumor, easily removed with a sharp pair of scissors.

Teeth.-(See Mouth Diseases.)

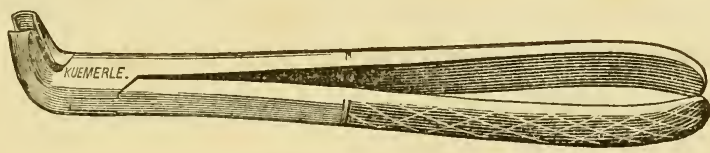

TOOTH FORCEPS.

Tetanus.-(See Lock Jaw.)

Tetter.-(See Skin Diseases.)

Thick Wind.-(See Heaves and Broken Wind.)

Thick Leg.-(See Grease and Farcy.)

Thiselo.-(See Fistula.)

Thoroughpin.-An affection of the hock-joint, accompanying bog and blood spavin, characterized by soft, fluctuating swellings, containing fluid or joint-oil in increased quantity, and altered in quality. 
Treatment.-The same as for bog or blood spavin, (which see.)

Thread Worms.-(See Worms.)

Throat Diseases.-(See Bronchitis, Cold, and Catarrh.)

Thrush.-(See Foot Diseases.)

Thrombus.-(See Jugular Vein.)

Thumps.-Is spasm of the diaphragm, or the curtain which hangs down and separates the cavity of the abdomen, or the belly from the chest.

Cause.-Over driving and oppression, paralyzing the accessory nerve, and hence the flapping of the diaphragm.

Treatment.-Place the horse in a cool, airy place, and allow the animal plenty of cold water, and if the noise after an hour or so does not subside, give two bottles of ale or porter as a drench.

Tongue, Laceration of.-(See Mouth Diseases.)

Tonics.-(See Medicines and Prescriptions.)

Toxicology.-(The science of poisons, and their antidotes.)

Tracheotomy. - An operation for to open the wind pipe, in cases of closing of that tube by tumor, or thickening of its membrane. It is an operation rarely required, and moreover, not to be attempted by a non-professional person not acquainted with the art and science of surgery. Therefore we will forego a description of it.

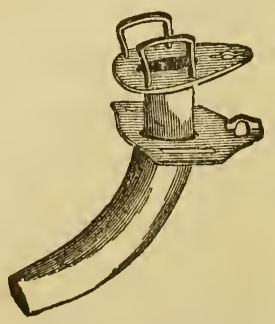

Tracheotomy TUBE. 
Transfusion.-This means the transfusion of blood from one living animal to another. Mr. James Farrel, of Dublin, Ireland, has been the first veterinarian I am aware of who have employed transfusion to any extent, and to excellent advantage. In cases of debility from blood letting, he has done wonders with it, and have proved to his countrymen the danger of bleeding horses to cure or prevent diseases. For in the cases which had not been bled he had no trouble with them; but in those which had been bled before he had been called to them, every one of them died. Hence he had resource to transfusion, in order to save others treated by bleeding. The effect of transfusion is instantaneous, almost.

Tread.-(See Foot Diseases.)

Trepaning.-This is an operation for opening into the bones of the head or face, for elevating or raising a portion of bone, which from accident has been depressed, or fractured. The instrument is called a Trephine, and

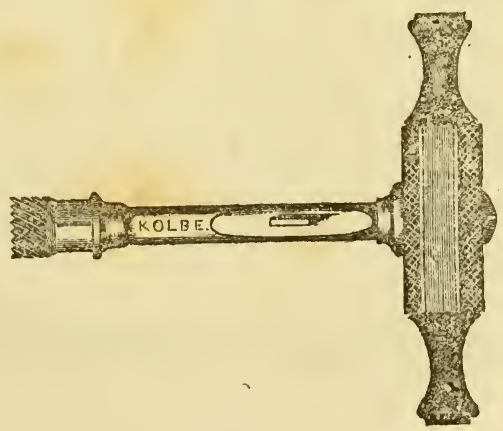

Trephine.

is represented in the accompanying drawing. This instrument is used to cut out a circular portion of the bone, so that a smooth piece of iron is inserted in the hole, and 
used as a lever to raise the depressed portion to its proper level.

\section{Trismus.-(See Lock Jaw.)}

\section{Tubercles.-(See Glanders.)}

Tumors.-Tumors may be defined as circumscribed swellings of different sizes, without inflammation, and differing from one another, according to their situation and their nature.

(1.) Encysted Tumors.-This is a variety of tumors often seen about the side of the nose of the horse, of about the size of a pullet's egg, soft, and elastic to the touch, with no heat, inflammation, or soreness of any kind.

Cause.-Closing of the sebaceous openings of the part. Hence the elevation, swelling, and filling up of that portion of the skin which contains the sebaceous or suet-like matter.

Treatment.-Open the part with a knife, and inject for a few times into the inside of the tumor a teaspoonful of the tincture of iodide, to kill the walls of the cyst. The surgeon sometimes dissects those tumors out, in the form of a soft ball, without opening them. This requires a steady hand.

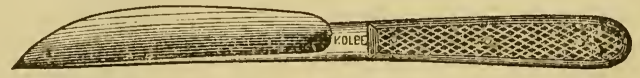

I1 OPERating Knife.

(2.) ENCEPHaloId.-This term means a brain-like tumor, so named from its structure being like that of the brain. It is one of the kinds of cancers which sometimes attacks the horse, but more often seen in the dog and ox. 


\section{TUMORS.}

Cause.-A specific poison in the blood, not as yet well understood.

Symptoms.-Solid, hard and irregular in shape, and knotty, with no hair upon its 'surface, and presenting a smooth and shining aspect. Those tumurs grow rapidly and finally break on the ${ }_{5}$ top, and present an angry and malignant sore or ulcer, which cannot be healed without first removing the tumor, entire with a knife, and treat it as for a simple sore.

(3.) Fibroma.-This is a fibrous or warty-like tumor, familiar to most persons, and is attached chiefly to the skin only. They are troublesome only so far as they are apt to bleed, and keep up a degree of irritation, especially in warm weather from the sweat, and the flies.

Treatment.-To those persons who has not sufficient nerve to cut them off with the knife, take arsenic, one drachm; hog's lard, four drachms. Mix, and make an ointment, and rub in and around the tumor, once a week a small portion of the salve, and in a short time it will fall off.

FatTy Tumor.-This is a variety of tumor of frequent occurrence in horses, and is composed of fat as its name indicates, and is sometimes called adipose and lipoma, by the surgeons of continental Europe.

Symptoms.-A round, ovoid shape, with a firm feel, but not hard nor elastic, or at least not so much so as the encysted form.

Treatment.-Removal with a sharp knife. Take a sharp knife, first have a twitch upon the horse's nose, and one of the fore legs held up, and cut a straight eut over the centre of the tumor through the skin only. Then roll out the ball of fat with the fingers, the knife here is of no use. By simply opening the skin, and rolling out 
the fatty tumors, this is called occlusion, and when cleverly done, looks well.

(5.) Cancerous. - (See Melanosis.)

(6.) Meluanoid.-(See Melanosis.)

(7.) Bony Tumors.-A species of tumors attacking the bone, and is called osteo-sarcoma, (which see) and when attacking the head, it is called osteoporosis. (Which See.)

(8.) VARICOSE.-The horse is not often the subject of varicose tumor of the veins, simply from the fact that no garters or other ligatures are tied around the legs, interfering with a free circulation. The saphena major however, is the only vein liable to varicose, and arises from bulging of the hock-joint, and in cases of bog, and blood spavin, and thorough pin. (Which see.)

Tymapanitis.-This term means drumbelly, such as occurs in cases of flatulent colic. (Which see.)

Typhoid.-A low form of disease, accompanied with fever, example, Influenza, or catarrhl fever. (See Influenza, and Gastritis Mucosa.)

Ulcers.-I do not intend to speak of internal ulcers, as of the brain, chest, or belly, as they are beyond the skill of the most learned, much less the non-professional reader, but will confine myself to external ulcers, as of the skin and flesh.

(1.) Healthy Ulcers.-These are generally the result of an accident, or incision with a knife, or other instrument. Every sore which does not heal by what is called the first intention, but suppurates, is called a healthy ulcer. 
Treatment.-Most healthy ulcers will heal of themselves: at most all that is required to be done, especially in warm weather, is to keep the granulations, (which see) from growing too fast, and above the level of the skin; and for this purpose apply a solution of blue stone, or chloride of zinc as follows:-Chloride of zinc, four grains; rain water, one ounce; mix. Or, powdered blue stone, two drachms; rain water, eight ounces; mix. One of these mixtures may be applied once a day, just suffcient to moisten the sore, and keep proud flesh down. The simple ointment of the drug stores is a good healing salve, and should always be in the house and at hand.

In neglected sores, and when proud flesh has grown up above the level of the skin of the healthy part, take a stick of caustic potassa, and hold one end with a piece of cloth, or stick one end in a goose quill, and touch the sore with the other end of the caustic a few times, till the proud flesh turns black. Repeat at another time, if necessary. Keep the caustic in a tightly-corked bottle, or else it will be dissolved into fluid when next wanted.

Sewing the lips of wounds and sores together is not now much practised, as the stitches are apt to rot, and the parts swell, and, consequently, the sewing gives way, and only aggravates the sore.

Adhesive plaster is also not advisable, as, when it is put on over the sore, it prevents the proper fluids of the sore from being discharged, and the moisture serves to loosen the plaster.

(2.) Indolent Ulcers. - This variety of ulcer or sore usually attacks the skin of the legs and heels of horses, (see Phagadena, Grease, and Farcy) eating down into or below the surrounding surface, and covered with a whitish-gray matter, and, in some few cases, small red 
spots are seen looking through the white covering. These are granulations, and are possibly at this time unhealthy ones.

Cause.-A bad habit of the body and blood, poor feeding, and debility.

Treatment.-Apply powdered blue stone to the ulcer, to eat off the unhealthy surface. Then apply a poultice for the night, made of any soft moist, material, say boiled turnips, carrots, or bran and flaxseed meal, made with a little warm water. The face of the poultice to be covered with powdered charcoal or brewer's yeast. Continue the treatment with an occasional poultice, and the solution of blue stone. (See Healthy Ulcers.)

Feed the horse well, and give half-ounce doses of the sulphite of soda once a day, to purify and enrich the blood.

(3.) Irritable Ulcer.-Example-sores on the pastern joint irritated by the flies, heat and sweat of summer.

Symptoms.-Cannot be touched without they bleed; angry-looking, and very sore; highly inflamed, and extremely vascular.

Treatment.-Difficult to cure during warm weather, but easily so in moderate weather, and with no flies.

Dress the sore with oil of olives, one cunce; creasote, half an ounce; oil of turpentine, half an ounce; mix, and apply to the sore with a piece of soft cloth, once a day. Do not let any of the mixture run down on the hair, which will, if so treated, fall off.

Urinary Calculi.-(See Stone in the Bladder.)

Urine Bloody.-(See Hæmaturia.) 
Varicose.-The enlarged vein on the hock-joint, caused by bog and blood-spavin. (See Spavins and Tumors.)

Venesection.-Bleeding by opening a vein.

Ventilation.-Few persons are aware of the vast importance of pure, fresh air, in the maintenance of health, and the prevention of disease, in both man and beast. However necessary pure air is in health, it is still more so to an animal when sick from fever and disease; and indeed there are diseases in which no treatment can or will be successful, no matter how well they may be treated, without pure fresh air and cold water to drink. Show me a badly ventilated stable or barn, and I will show you in the spring of each year horses fevered and diseased. (See Disinfectants.) Coughs, colds, lung fever, influenza, grease, scratches, farcy and glanders, are the results of bad ventilation.

Who has not heard with horror of the BLACK HoLE AT Calcutta, in which one hundred and forty-six men were confined for a few hours without ventilation, and only twenty-three survived the short confinement. Horses confined only for a few hours without ventilation, as was the case in two military expeditions sent out by Englandone to Quiberon, and the other to Varna, in which the hatches of the ships were put down, and only for a short time, but sufficient to produce glanders in almost every horse so confined. Hence, it will be perceived that, without good ventilation, a high standard of general health cannot be maintained very long.

\section{Veterinary Biography.}

The increased facilities for receiving and transmitting intelligence to and from all parts of the world, make it 
an object in a book, designed, as this is, for the non-professional reader, that he should know something of the persons, whose sayings and opiniuns he has received, concerning the cause, treatment and prevention of diseases of stock, throughout the world. The history of veterinary medicine, has a claim to greater antiquity even than that of domestic medicine, which few will deny; or else why should Vegetius have uttered these words:- "Ars veterinari post medicinum Secunda est" and write a book in VIII volumes, entitled "De arte Veterinaria." And further, we are assured of this by concurrent testimony, through the writings of Homer, 1000 years before Christ, that this science had an existence in his day, and corroborated by Herodotus 500 years later. Simon, the Athenian Farrier, was practicing the art as. it then existed, fully 300 years before Galen, and about 500 years before Celsus, the Roman physician, had yet lived. However, by order of the Greek Emperor, Constantius, of the seventh century, the various writings upon the subject were collected, and arranged under the title, Hyppoeratika, and were translated from the original Greek into Latin, by Dr. Ruelle, in the latter part of the fourteenth century, by order of Francis the First, King of France. Ramazzini, of Italy, Sauvage, of France, and Camper, of Germany, form the connecting link between ancient and more modern times, or till the advent of Charles Vial de Sain Bell, the founder of the first veterinary college in England, in the year 1792. The first being established at Lyons, France, in 1761. Sain Bell was a man of ability, a good reasoner, clever, and like his countrymen, very brilliant. He died before much progress had been made in establishing the college. He was succeeded by a dashing young medical student named Coleman, a favor- 
ite of Sir Astley Cooper, the great surgeon; but it is by no means clear that he was so regarded by the celebrated John Hunter, as filling the chair of so illustrious a predecessor. However, he was the means by which veterinary science made considerable progress.

The grave has, some years ago, closed over his mortal remains; but his writings are much thought of, and quoted by some, although other writings, more modern, exist. Where there was but one great teacher, there are now many, and able. Among them was Wm. Youatt, known wherever the English language is spoken. A clear and indefatigable writer, a great and constant advocate of bleeding, and blistering, in nearly every disease; however inadmissable, many persons and publishers have tried their hands to elevate his works to the new idea of a more rational and successful system; yet, withal, much inconvenience and loss, to the agriculturist, results from consulting his works, when sickness overtakes their stock. He was a bold and daring man. Many times he allowed himself to be bitten by mad-dogs; and, strange to say, he never suffered from canine rabies.

James Beart Simmonds, who is still living, is known in this country by his report on the rinderpest to the British Parliament, professor of cattle pathology to the Royal Veterinary College, and a man of ability.

Charles Spooner, principal of the college known to the readers of the Royal Agricultural Society's Journal. The Scotch are well represented by Professors Dick, Dun, and Gamgee. Mr. Dick is the founder of the present Edinburgh College, and veterinarian to the Highland and Agricultural Society. Mr. Dun is sound, and learned, and has carried off more prizes on veterinary-agricultural subjects, than any other man. Mr. Gamgee may justly 
be called the Napoleon of veterinary surgeons; his writings, though scientific to a fault, are not for the ordinary reader. His work on the rinderpest, reflects much upon him for his learning, and ability. The Irish agricultural interests, are protected by Messrs. Ferguson, and Farrel. Edward Mayhew is an invalid, and has confined himself to book-making, and he has shown the world well, how a man may write books, when he knows nothing of the subject he is writing about. In his works, the bookmaking tact is seen to advantage; yet, withal, his works bear many evidences of the cleverness of a "Mayhew."

The French are well, and creditably represented, by Reynal, and Boulay; and Germany, by Herring. The United States has made great progress in veterinary science, within these few years, and especially in regard to the treatment of disease. "The Massachusetts cattle disease" seems to have shaken the faith of the people in the writings, and recommendations of Dr. Dad; and for R. Jennings; he has spoken for himself. The various state agricultural societies, and farm-schools, are doing much, in the way of advancing the cause of a rational system of animal medication, throughout the land. The Philadelphia Society, for the Promotion of Agriculture, the oldest society of the kind, in the country, has long been a powerful patron of veterinary science.

The Pennsylvania Farm School, orders a course of lectures to be delivered before the class, each session. The New York State Agricuitural Society's efforts are gigantic, practical, and in the right direction. The secretary, B. P. Johnson, Esq., is well known throughout the country, as devoting his life to the cause, and advancement of agriculture, in all its auxiliary branches. Mass̉achusetts has no less spoken, through Mr. Flint, her secretary. 
Ohio, and other states of the Union, might be named in this connection, but space will not permit.

Veterinary Colleges-Do not seem to flourish in this country; the one at Boston has long ago closed its doors. In Philadelphia, the "Merchants' Veterinary College," bids fair, however, to be a success, under the guidance of Prof. Robert McClure, to whom all inquiries, in regard to the college, should be addressed. Diplomas are issued on the first of March, each year, to students, and honorary members.

Vives.-A term given to bastard, or an irregular variety of strangles. (See Strangles.)

Warts.-(See Tumors.)

Warranty.-A form of certificate given on the purchase or payment for a horse. There is nothing mysterious, nor yet of much importance, as to the form of a warranty. The best forms ammount to nothing, in the eyes of the law. Horses are like other merchandise. If not as represented, damages or difference in value can be obtained by a process of law, providing the seller can be found, and whether he has property which can be levied upon. 'Tis true, in such a case, an action in tort may be brought whereby if payment is not made, he can be sent to prison for awhile, or till he can claim the benefit of the insolvent act.

Few persons will bring an action at court for swindling, or getting money under false pretenses, by horse dealing, which action will be difficult to maintain, and get a verdict upon it. (See Soundness.)

Warbles.-(See Skin Diseases.)

Water Farcy.-(See Dropsy and Farcy.)

Weeping Eye.-As its name indicates is a flow of 
tears from the eye down the side of the face, instead of through their proper channel.

Cause.-Obstruction in the lachrymal sac, or nasal duct, from a disease called fistula lachrymalis.

Treatment.-This is the province of the surgeon and anatomist.

Wens.-The common name for external tumors.

Wheezing.-This is a sound given from the horse, having enlarged glands, or thickening of the membrane of the wind pipe, or the glands pressing upon its head decreasing its calibre. Whistling is caused by the same alteration of structure in the wind-pipe.

Whirl-Bone, a Sprain of--(See Sprains and Hock.)

\section{Wind-Sucking.-(See Crib-Biter.)}

Worms.-The worms which inhabit the body of the horse are of many varieties. Some of them are harmless, and the others interfere with the health of the horse. 1st. The bot or oeestrus equi, found inhabiting the stomach. 2d. The oestrus Homorrhoidalis or Fundament bot found in the rectum, and often seen about the anus, and under the tail. 3d. The strongylus, and Filaria, found in the aorta, and other blood vessels. 4th. The ascarides vermicularis, found in small cells within the mucous covering the cœcum, or blind gut. 5th. Filaria, found in the aqurous humour of the eye.

(1.) Sтомасн Вот.-These worms are the result of turning horses out to pasture in the summer months, and are produced from the eggs laid or glued to the fore legs of the horse, by the bot fly.

Symptoms.-An unthrifty coat, and loss of flesh after 
a run at grass, may be taken as an indication that bots are present within the stomach.

Treatment.-Improve the condition of the horse, so that the debilitating effects of the bot's presence may not interfere with the general health and condition of the horse, for it must be born in mind that no medicine can, or will, dislodge or destroy those parasites short of killing the horse, also. Once the eggs are into the stomach, which is the natural nursery for their protection, and propagation of their species, therefore they cannot be removed by force. In one year from the time the eggs are taken into the stomach, will the bot be a perfect chrysalis, and will fall from the coats of the stomach, and be expelled with the excreta or dung, and in a short time after they will be provided with wings, and fly about and commence the propagation of their species, which pass through the same period of probation or incubation, as the original progenitors of their race. Give iron and gentian, in addition to good feeding, to prevent the bots from delibitating the animal too much. Powdered sulphate of iron and gentian root, each three drachms. Mix, and make one dose, to be repeated twice a week,

(2.) Fundament Bot.-Like the stomach, but are also the result of a run to the grass; but instead of the eggs being deposited upon the legs, they are stuck to the muzzle or lips of the horse, and are the color of the skin, hence not often seen.

Symptoms.-The following year, during the summer months, the larva of this species will be seen sticking about the anus, and under the tail, which in spirited horses prove a source of great uneasiness and irritation.

Treatment.-Injections of linseed oil, or tobacco smoke. 
(3.) Strongruds.-This variety, and a species of Faliria, are sometimes found in the blood vessels, and are similar in the effects produced in the horse, to those seen in sheep affected with rot.

Cause.-Feeding on wet and marshy land, and pasture having been flooded with water. Who has not heard of the effects produced from this cause, in animals grazed upon the course of the river Nile, in Egypt after each inundation?

Treatment.-Support the strength by good, generous feeding, and give iron, and gentian, each two to three drachms once a day, and remove the animal to high and dry pasture.

(4.) Ascarides.

Cause.-A bad habit of body, called cachexia and chlorosis.

Treatment.-Give iron, gentian, arsenious acid, in the following manner: powdered sulphate of iron, two drachms; gentian root, two drachms; arsenic, five grains. Mix, and give in one dose in mixed, cut or soft feed, twice or three times a week.

Wounds-Are divided into simple, incised, contused, lacerated, punctured and poisoned. Wounds are dangerous, when entering into cavities, as the chest and belly, and from poisoned wounds, or from the bite of a mad dog. (See Bites.)

Wounds following by bleeding, will be found treated of under the article bleeding wounds. (Which See.)

Contused, lacerated and punctured wounds are generally followed by suppuration, (Which See) which should be encouraged by warm poultices applied to the parts, and open the parts freely, to allow the pus free 
escape. Wounds entering the belly or chest, should be treated by placing a pad over the part to exclude the air, and application used as for simple wounds. Keep down pain by giving twenty drops of the tincture of aconite root, three times a day, for two days only. Poisoned wounds will be found treated of under the article bites of mad dog. (Which See.)

Wourali.-A name given to a poison, which is prepared by the Macousi Indians, of South America, and used by them on the points of their arrows. This poison has becn advocated by some in the treatment of locked-jaw, in the horse, but in my hands nothing favorable can be said of it as a cure for this terrible disease. The power of this poison is so great, that an ox, of one thousand pounds weight, was pierced in each thigh with an arrow, poisoned with it. The poison took effect in four minutes, and in a few minutes more his head and legs ceased to move, and in twenty minutes from the time he was wounded, the ox was dead, and apparently dying without pain.

Yellows.-Discoloration of parts of the skin from liver disease. (See Liver.)

Yellow Water.-(See Liver Diseases.)

Zoology.-The natural history of animals.

Zumins.-(See Ferments.) 

PART II.

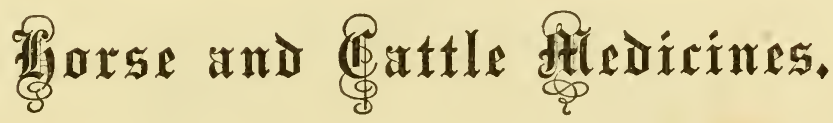





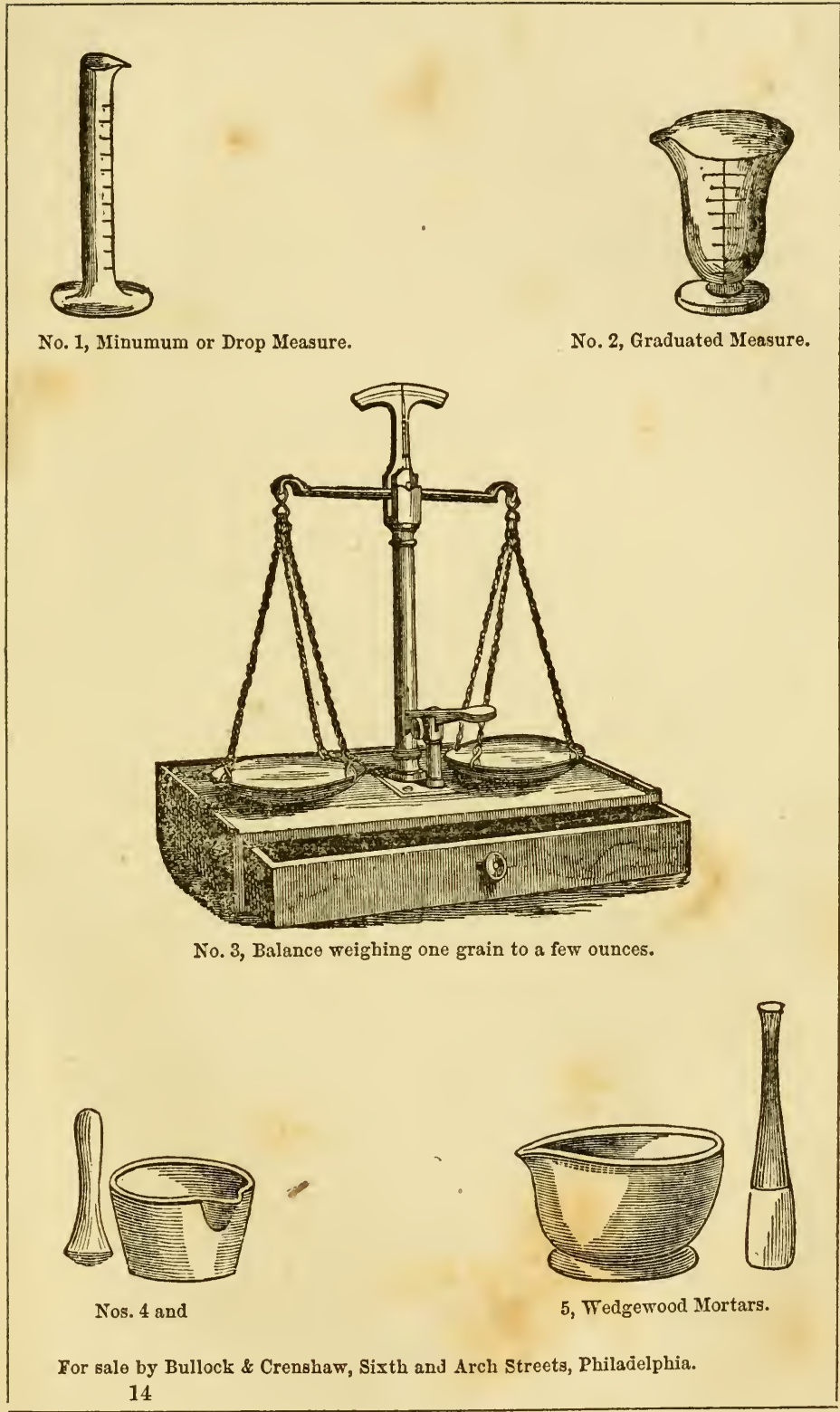





\title{
HORSE AND CATTLE MEDICINES.
}

\author{
PART SECOND.
}

IN the language of one of South Carolina's learned sons, who has long and honorably filled the chair of Therapeutics in the Jefferson Medical College of Philadelphia, "Give me mercury, iodine, quinine and the lancet, and I will combat with disease." Without altogether endorsing the views of this distinguished professor, either in the choice or numbers of medicines he thinks necessary, or sufficient to cure disease, I would say, give me aconite, iodine, iron, mineral acid, soda, creasote, and a few others, and I will not only combat, but successfully cure and overcome disease. Every article in use by myself in the treatment of diseases will be described under the name it is best known. No attempt will be made to describe adulterations in the drugs spoken of. Many useful plants containing high curative properties are to be found all over this country, which will be well worth paying attention to.

\section{Acids.}

These are derived from the mineral; vegetable and animal kingdoms, and are of a sour taste: whence the name acid. Acids are solid, and some fluid, and all are 
easily dissolved in water. Acids are mostly poisonous, except when highly diluted, or mixed with with water.

ACETIC ACID.-This acid is eight times stronger than ordinary vinegar.

Use.- Sometimes used in sprains, and for the destruction of the poison of insects, by adding one ounce of camphor to four ounces of the acid.

Pyroligneous ACID-is got by the distillation of rood, and sometimes sold in a diluted form as white vinegar, and is used with salt by horsemen to sore backs and shoulders.

Muriatic AcID.-This is commonly called the spirit of salt.

Use.-A good tonic in debilitating diseases in horses and cattle, and is much used by me in pleuro-pneumonia in cattle, for it relieves the quick breathing, and keeps up the strength.

Dose.-Forty to sixty drops given largely diluted, or mixed with cold water, and repeated three to four times in the day.

Externally, it is used for sores in the feet of horses, by pouring a few drops in the nail-hole or sore. Ten drops poured into the fistulous openings of poll evil, or quittor in the foot, daily, sometimes cures the disease.

Nitric Acid, or Aquafortis.-This given properly, and largely diluted, is an excelent tonic, and is used for the same purpose, and in the same doses as the preceding.

Sulphuric AcId.-Possibly this acid is preferable to any other of the acids for internal use, in weakness and debility, and is given in from forty to sixty drop doses, also largely diluted with water. Horses and cattle will 
usually drink water, when acid is in it. In all kinds of fevers, sulphuric acid is an excellent medicine to give. In mixing this acid, the acid is to be added to the water, not the water to the acid.

Prussic AcID. - A highly dangerous poison. There are two forms of this acid kept in the drug stores. Scheels contain five per cent., and that of colleges about two per cent.

Use.-Given in locked-jaw in the horse with considerable success.

Dose.-Half to one drachm once a day. If the horse be young, half a drachm will be enough. Two drops to the ounce of water is an excellent wash to the skin of all animals, in mange and inveterate itching. Great care, however, must be exercised in its use, whether used internally or externally. It is sold under the name of hydrocianic acid.

Aconite.-(Aconitum Napellus,) wolfsbane, monkshood.-An active poison, and one of the garden plants of parts of Europe. The tincture is that portion which is used in diseases of horses and cattle.

Tincture of Aconite root-is one of the most powerful, certain and successful sedatives which can be used. It has done away with bleeding, blistering and physicing, which were formerly thought proper agents wherewith to combat and cure disease. It is not only sedative, but it is a nauseant, calmative, anodyne, stimulant diaphoretic and antiphlogistic. It controls fever, and allays pain and inflammation; and is the only medicine, excepting hellabore, which can excite the horse, the ox or the elephant, to vomit. Although these animals cannot vomit, it is the one to cause them to make the effort. If, according to the founder of homopathy, mercury was a 
divine metal, no less surely may aconite be considered a divine regetable; for without it, or something as good, what can be so successful in curing lung fever, founder, inflammation of any part of the body, colic from eating green food, fever accompanying cattle diseases of whatever kind, nothing controls the circulation and action of the heart so promptly as aconite. The nervous centres of the body are no less ready to obey its action Hence, its value in allaying fever, irritation, excitement and pain, from whatever cause.

How to use it.-Aconite should never be carried too far, or prostration and weakness will follow. Never give more than eight doses, when twenty drops are the dose, nor six doses when twenty-five drops are the dose. Or, in other words, never, in any disease, give more than two drachms, or one hundred and twenty drops, whether the dose has been ten, twenty or twenty-five drops. In pleuro-pneumonia and other diseases, iron and the mineral acids should follow aconite.

Alcohol.-Spirits of wine entirely free from water, and is used for making tinctures of the various plants, and is the excipient or foundation to many lotions ard liniments. Alcohol may be given to horses having a chill, in half pint doses, mixed with a little warm water, not too hot.

Aloes.-This is the expressed juice of several plants, of the name. Aloes are of several varieties:-Barbadoes, Socotorine, Cape, and Hepatic; all of which differ in color, and some of them in strength. Barbadoes, and the Socotorine, are the varieties kept in the drug stores.

Use-Aloes is used as a purgative, or cathartic, for horses only. Cattle do not respond to aloes, nor is it a proper internal medicine for those animals. 
Dose.-From six to eight drachms, made into a bolus, or ball, with two drachms of powdered ginger, and molasses, to cause them to stick together.

Tincture-of aloes is an excellent application to a healthy sore. In the form of compound tincture, which is composed of aloes, and myrrh, and is preferred, by some persons, to the simple tincture.

Alum.-A compound salt, containing sulphuric acid, potash, and alumina.

Use.-Applied to sores, to arrest bleeding, and in the form of burnt alum, is used to touch indolent sores, which are not disposed to heal quickly.

Ammonia. Liquid ammonia is commonly called spirits of hartshorn.

Use.-A diffusable stimulant, and is given in cases of flatulent colic, and formerly was in much repute by farriers, mixed with oil, as a liniment, but which is not now considered of much value, and is apt to blemish.

Dose.-Two drachms to half an ounce, as an anti-spasmodic, in flatulent colic.

Carbonate of Ammonia.-A highly valuable medicine, when given in weakness, debility, and prostration, from influenza, lung fever, bronchitis, etc.

Dose.-From two drachms, to half an ounce, and may be repeated three times in the day, and should be given, mixed in cold gruel, so that the latent, or hidden heat, will not be developed, as it would be, if given in any thing warm, and thereby scald the throat while drenching.

Muriate of Ammonia-possesses the same properties as the above, but is not so strong.

Acetate of Ammonia-is made extemporaneously, by dissolving the carbonate in strong acetic acid, till effer- 
vescence ceases; then, adding water to it, till it is of the strength of ordinary vinegar.

Use.-An excellent form of ammonia, when given in weakness, and debility. Indeed, the ammonia, and its preparations, need only to be used, to be convinced of their utility, in supporting the vital powers, and thereby curing disease.

Anæsthetics.-Medicines which induce insensibility.

Aniseed.-A warm carminative, and used in conjunction with fœnugreek, and cardamoms, in weed, and fevers in milch cows, and for indigestion, and loss of appetite in horses.

Dose.-For horses and cows, one to two ounces, given three times in the day.

Anodynes.-These are medicines which allay pain. (See Aconite, Opium, Morphia, Chloroform, and Ether.)

Antimony.-A metal. Many preparations of antimony are kept in the drug stores.

Tartarized Antimony.-This is commonly called Tartar Emetic, and was formerly relied upon in the treatment of lung, or chest diseases in horses, and cattle; but, to the great surprise of the knowing ones, recent experiments have shown that tartar emetic has no sedative effect whatever, upon horses, cattle, sheep, or other herbivorous animals. Pounds of tartar emetic have been given to horses, cattle, and sheep, without any effect, excepting increasing the appetite a little, and having no effect upon either the respiration, or circulation, whatever. Therefore, tartar emetic, we will leave to the dogs, and to man.

Tartar emetic ointment is sometimes recommended, to be applied to the region of the kidneys, where the Spanish fly dare not be used. 
Ter Sulphuret—of Antimony, or Black Antimony.This is a favorite, with many horsemen, for making a horse shine. (See Prescriptions.)

Ter Chloride- of Antimony. - This is a useful medicine, for diseases of the feet of horses, cattle, and sheep; it owes its properties to hydrochloric acid. It is commonly called, by old farriers, buttyre of antimony. In foul claw, in cattle, and sores in the feet of horses, a piece of cotton should be moistened with it, and laid upon the sore.

Antiseptics.-Medicines which arrest decay, and putrefaction.

Antispasmodics.-Medicines capable of relieving spasms; as in colic.

Areca Nut.-An effective cure for tape-worm in the dog.

Dose.-About two grains of the nut, to every pound of the animal's weight, and give in the form of a pill, or suspended in milk.

\section{Arnica Montana.-Leopard's bane.}

Tincture of Arnica.-A valuable remedy for sprains and bruises. A lotion is made as follows: Tincture of arnica, one ounce; water, two ounces; cloths, kept wet with it should be laid on the part which is sore.

Arsenic.-A well known poison.

Use.-A splendid tonic, improving health, condition, and wind of the horse.

Dose.-When given once a day, five grains will be a safe dose, for an ordinary sized horse. If given twice a day, two and a half grains will be enough.

Arsenic should be given mixed in feed, in large bulk, such as cut feed, so as to protect the stomach as much as possible, whilst at the same time we reap its advantages. 
These doses should be continued at least for two weeks, stopping at that time for one week, and commence again as before. This will insure safety, with the benefits of the arsenic, in an improved condition in both wind and body. In Switzerland, and other parts of the world, arsenic is relied upon for the cure of heaves in the horse.

Fowler's Solution of Arsenic.-Made as ordered by the colleges, contains four grains of arsenic, to the ounce of the solution, or fluid.

Asafœetida.-This is a gum-resin, having a smell like onions. Asafoetida is a stimulant and vermifuge, and is given to horses for loss of appetite, and to produce a fine silken coat upon them. It is given to dogs, to expel worms. Dose, for the horse and the cow, two drachms, and for dogs, ten to twenty grains.

Tincture.-Where the stimulant effect is wanted, the tincture is a good form, and is given in about the same doses as the gum, for all animals.

Asarabacca.-This is used in horses, where matter is lodged in the nares of the nose, and it is desirable that it should be brought down, and also in sheep and calves, with faliria bronchi, or worms in the air passages. For horses, blow a drachm up each nostril, out of a cone of paper, taking care that the person does not inhale it himself. For a flock of sheep, or a number of calves, confine them in a close shed, with sufficient air, and throw a considerable quanity among them, so the animals will inhale it. Sneezing and snorting take place, followed by mucous, and small worms, from the nose. This action is called errhine.

Astringents.-Medicines which are capable of drying up discharges, whether from the bowels, or from a 
sore. The principal medicines of this class are opium, tannin, oak-bark and chalk.

Atropine.-The active principle of Belladonna. (Which See.)

\section{Belladonna.-Atropia Belladona.}

Belladonna is used in veterinary practice for the same purposes as opium, but is to be preferred to opium on account of its having no binding effect on the bowels. The extract is the preparation used, and is prescribed in all animals, for colic, rheumatism, coughs, sore throat or bronchitis, influenza and locked-jaw. Doses, half a drachm to forty or fifty grains dissolved in half an ounce of the sweet spirits of nitre, and repeated three times a day, in the diseases above mentioned. In diseases of the eye, it is considered valuable, as it has the power of contracting the iris of the eye, thus causing enlargement of the pupil, and breaking up adhesions between the iris and the lens. It also facilitates operations on the eye.

Benzoin.-A gum-resin, the tincture of which is known as the old Friars Balsam, and is an excellent application to sores, and unhealthy ulcers.

Benzole.-This is a watery carbon, and is obtained from coal tar. Use. To destroy lice, and other insects in the skin of all our domestic animals.

\section{Bismuth, Subnitrate of.}

Use. For dogs when affected with vomiting, and purging. Dose, Five to fifteen grains, given on sugar, and may be repeated.

Bole Armenia.-Formerly in great repute by old horse doctors, for many diseases of all the animals, and entered into almost every mixture. It is useless. 
Borax-Or Biborate of Soda. A good application for ringworm, for which purpose dissolve half an ounce of borax in eight ounces of water.

Bromide of Potassium.-Use. For arresting the growth of fibrous tumors which are so often met with in all animals. Doses, For horses and cattle, from one to three drachms, and for dogs, five to ten grains, repeated three times in the day, for a considerable period.

Buckthorn.-A sirup is made of the berries, and kept in the drug stores, and is recommended as a mild purgative in dogs having distemper.

Calamine.-An impure carbonate of zinc. Oxide of zinc should be used or substituted for it. Used in the healing of wounds, both in powder and ointment.

Calcium, or Quicklime.-Use. Lime is used in indigestion and acidity in all animals, and in tympanitis in the horse, and hoven in cattle, and in the form of lime water, and mixed with linseed oil in equal quantities makes the celebrated carron oil, so called from its constant use among the men employed in the carron iron works, when any of them get burnt. Lime water and calomel makes the yellow wash, and corrosive sublimate, and lime water, make the black wash, so highly recommended in unhealthy, and in ill-conditioned ulcers and sores. It is used for a disinfectant in stables and barns. Dose. Quick lime is given to horses and cattle, in from one to two drachms. Lime water is given in five ounce doses to horses and cattle. For calves with diarrhœea, two ounces of lime water, a drachm of powdered gentian root, and from three to five drops of the tincture of aconite root, given three times a day, is an excellent remedy. 


\section{Calomel.-Chloride of Mercury.}

Calomel should be rarely used in horse and cattle practice, from the fact that animals are not so subject to scrofulous diseases, and liver complaints, as man is, and as those are the diseases chiefly calling for calomel, we can in veterinary practice do without it, so far as internal use is concerned. For external application, in the form of the black and yellow wash, it is valuable. (See Prescriptions.) Calomel sprinkled on unhealthy ulcers and sores is valuable, quickly changing their appearance. Calomel is sometimes used in thrush of the foot of the horse, inserted into the cleft of the frog. In cattle with foul claw, and in sheep with sore feet, it can be used to great advantage. A dog can take as much aloes as will kill a man, but a man can take as much calomel as will kill several dogs.

Camphor.-Use.-Camphor is a slight stimulant, followed by calmative effect. Hence, it is recommended in cases of irritation, nervous excitement, and enters into all mixtures used in cases of chronic cough. Three to five grains of camphor, and extract of belladonna five grains, rubbed down in a little whisky or alcohol, and mixed in three ounces of water, is an excellent remedy in allaying irritation in dogs having distemper. An excellent rubifacient can be made with camphor, one ounce acetic acil, No. 8 , four ounces. To allay irritation in a sore, camphor and sweet oil-is a good application.

Doses.-Camphor is given to horses and cattle, in from two to four drachms, repeating it three or four times a day.

Cantharides.-(See Spanish Fly.)

Capsicum.-(See Pepper.) 
Carbolic Acid.-This is the product of coal tar, and closely resembles creosote in medical virtues. This is the chief part in the celebrated McDougall's disinfecting powder, so highly prized in England.

Use.-For checking long continned diarrhoa in cattle and sheep. Doses.-In its solid or fluid form, caroolic acid is given to horses and eattle, in from twenty to forty drops or grains. For sheep, five to ten grains or drops.

External Use.-For sores and wounds, when unhealthy, a good application may be made by adding one drachm of the acid to one pint of water. This will destroy all putrefaction, and induce the wound to take on a healthy action.

Cardamoms.-The seeds of a plant.

Use.-Given to milch cows in case of loss of appetite, and fevers. Generally it is united with foenugreek, ginger, or gentian root, mixed in warm ale, or molasses water. This mixture will cure ephemeral or fevers of a day's duration, when other medicines have failed.

Dose.-One to two ounces.

Carraway. - The seeds of a garden plant, and used for the same purposes as the preceding.

Carbonate of Lime.-Chalk is sold in the drug stores under the name of creta preperata, or prepared chalk, and is used for the same purposes as the preceding, but in larger doses.

Doses.-For horses and cattle, one to two ounces are the dose. For calves, two to three drachms, given in wheat flour, gruel, with a drachm of ginger, or carraway seed, to prevent griping.

Cascarilla.-The bark of a tree. 
Use. A bitter tonic, and resembles in some particulars that of quinine.

Doses. For horses and eattle, one to two ounsz, mixed in gruel.

Castor Oil.-The expressed oil of the seeds of the Ricinus Communis.

Use. However useful castor oil may be as a purgative in the human family, it is certainly a dangerous poison when given in sufficient quantities to induce purging, in either the horse, cow or sheep. It produces irritation, and inflammation of the coats of the bowels, without relief from purging.

For the dog, castor oil may be a proper and useful purgative, and to the pig, also. Aloes, and linseed oil, is the purgative for the horse. Epsom, or glauber salts, for the $0 x$, and the sheep. Whatever suits man, as a purge, will answer for the dog and pig.

Cassia.-False Cinnamon.-Much cheaper than cinnamon, and may be given to horses and cattle, in powder, mixed in gruel, for loss of appetite.

Dose.-One to two ounces, for horses, or cattle.

Catechu.-An extract from a species of acacia.

Use.-An astringent, binding the bowels in diarrhoa, and is used in combination with chalk and opium.

Dose.-For horses and cattle, two to five drachms. For sheep and swine, one to three drachms. For the dog, grains, ten to twenty.

For supperpurgation in horses, and cows, my favorite combination is as follows: Catechu, two to five drachms; prepared chalk, one to two ounces; powdered opium, ten to thirty grains; mix, and drench with wheat flour gruel. 
Chalk.-(See Carbonate of Lime.)

Chamomile.-The flours of anthemis noblis.

Use.-A very mild tonic, possibly too much so for the horse, or cow. We have better ones, although, perhaps, not so well known.

Charcoal.-Occasionally given to cows, in chronic diarrhoea.

Dose.-Half an ounce to one ounce, given suspended in gruel, of any kind. Externally, charcoal is very valuable, when applied to badly smelling wounds, and ulcers. It immediately corrects the foetor, and rapidly disposes them to heal. Charcoal and brewers' yeast, are good cleansers of putrid sores, and ulcers, and are worthy of more extended use.

\section{Chenopodium Anthelminticum.-Wormseed.-} An excellent remedy for worms in dogs, by dropping from two to five drops of the oil in a little soup, or from ten to twenty grains of the bruised seed, given for four successive nights, and then followed by a dose of castor oil.

Chlorine Gas.-Chlorine gas is prepared by pouring hydrochloric acid, on the black oxide of manganese, also by heating sulphuric acid with common salt and the manganese.

Use.-This gas is a disinfectant; and for this purpose it is made, and used as follows: Take an ounce or so, (depending upon the size of the place to be disinfected,) of black oxide of manganese, and hydrochloric acid, of sufficient quantity, carry them to the place where they are to be used, and pour the one into the other, "and close the doors, having first removed all the animals out of the place. A spirit lamp, placed under the bottom of the 
vessel, holding the materials, will insure a greater volume of gas. (See Disinfectants, in the body of the book.)

Chlorine, when sufficiently and properly used, is considered to be of great advantage, in arresting the ravages of glanders, farcy, and other distempers in the horse, and pleuro-pneumonia, and contagious typhus in cattle, and small pox in sheep. It is to be hoped that farmers generally, will provide themselves with proper apparatus, for this purpose, which will not cost above three dollars, and consists of a small lamp, with a stand so formed, that a small, glass bottle, commonly called a Florence Flask, can sit right above the blaze of the lamp, issuing from its wide, and open mouth, the disease-healing, and health-restoring gas.

Chloric Ether.-Chloroform, dissolved in spirits of wine.

Chloride of Potassium-Is similar to chloride of soda, or common salt.

\section{Chloride of Lime.}

Use.-As a disinfectant, from its antiseptic, and deodorizing effects, and is an excellent stimulant to unhealthy ulcers. Chloride of lime has been highly recommended in tympanitis in the horse, and hoven in cattle, arising from eating wet clover.

Dose. From two to four drachms, given, mixed with cold water.

As a disinfectant, it may be sprinkled on the stable, or barn floor, every morning; but a good way would be to suspend it in a box, having many small holes in it, and, hung from the roof of the house. If the house be large, two or more boxes may be used.

Chloroform-Terchloride of Formyle. 
Use.-Chloroform is an excellent stimulant, when given to horses having a chill, or shivering fit, from congestion, or from cold, and is equal to turpentine, for the cure of colic. An excellent liniment is made, by adding one ounce of chloroform, to two of olive oil.

Dose.-Chloroform is given to the horse and cow, in doses from one to two drachms, mixed in weak whisky, and repeated every two or three hours, or till the colic is relieved.

Inhalation.-The inhalation of chloroform, by either horse, or ox, is attended with risk, provided the animal be not secured, or tied, so that it cannot get loose; because some horses, and cattle, become completly wild, when the effects of the inhalation commences to act upon the brain. On the other hand, some horses will quietly stand up, others, as quietly, will lay down, under its effects.

Two to four ounces are sufficient to produce anasthæsia, or loss of sensibility. The usual way of giving chloroform by inhalation, is, by pouring about two ounces of chlorofarm, on a soft, and moist sponge, whilst the animal is tied down, and hold the sponge to one nostril only, covering the nose loosely, with a large towel, to save the fumes of the chloroform; but not too tightly, to exclude pure air from being admitted, with the fumes of the chloroform.

In all operations, lasting any length of time, whether in the horse or the cow, humanity and fine feeling, demand the outlay for a little chloroform. It is not necessary to completely destroy all feeling-just sufficient to blunt the sensibility of the nervous centres.

Chologogues.-Medicines which increase the flow of bile. 
Examples: Calomel, podaphyllin, irridin, leptandrin, etc.

Cinchona.-Peruvian, or Jesuit Bark. There are several barks of cinchona, used in medicine, and from which the sulphate of quinine is made. Cinchona, or its barks, are not used in horse and cattle diseases.

Quinine-is the only preparation used; but its high price is against its general use, in veterinary practice. But in valuable horses, it is used to hasten recovery from influenza, lung fever, etc.

Dose.-Twenty to forty grains, repeated from three to fonr times a day.

Quinine is apt to be adulterated with arsenic, as was the case with many samples, used during the late war.

Cochineal.-An insect, and used as a dye for coloring tinctures, and other medicines, for the purpose of deception. Druggists color water with it, for filling show bottles, which are placed in the store windows.

Cod Liver Oil.- In all animals, cod liver oil increases fat and flesh. In diseases affecting digestion, and assimilation, cod liver oil can be of no use. It materially relieves broken wind, or heaves, in horses. For hastening, or forcing animals intended for show, cod liver oil is just what is wanted, as it not only hastens the fattening process, but increases the quality, and appearance of the meat.

Dose. For swine, two ounces daily. For small pigs, one ounce.

Colchicum.-Meadow Saffron. The seeds, and root, are the parts usually employed in medicine, sometimes in powder; but the best is in the form of tincture. 
Use. Given in all rheumatic afflictions of the joints, and in lumbago, and also in diseases of the eye of the horse, depending on rhẹmatism of that organ.

Dose-Of the crom or seeds in powder for horses and cattle, the dose is from one to two drachms, given in the animal's feed twice in the day, for a week or two. The wine of colchicum will answer for the dog, in from five to fifteen drop doses, twice a day in a little water.

Collodion.-This is a solution of gun-cotton.

Use.-Collodian is used for dressing wounds and cuts, instead of sticking plaster, and is applied in the following manner:-Clip the hair from the edges of the wound, and take a camel's hair pencil, or a soft brush, and paint the surface and edges of the wound well, and in a few minutes, the ether, which holds the gun-cotton in solution, evaporates, and leaves over the sore a complete covering, resembling the gold-beater's skin, thus completely shielding the sore from the action of the air. Hence, its value.

Copper, Sulphite of.-Blue Vitriol.-This is one of the most useful articles that can be used in horse and cattle diseases, both internally and externally. Internally used, sulphate of copper is a powerful tonic, and builder up of the system, and is recommended in all diseases characterized by a low state of the system. Farcy, glanders, purpura, etc., in the horse, have, in several cases, yielded to its effects. Sulphate of copper should never be given alone, but should be combined with gentian or ginger. After the fever has passed off in cases of pleuro-pneumonia, the sulphate of copper is an excellent medicine to support the vital powers, and prevent effusions in the chest, which is the cause of death, in most cases, from this disease. 
Dose.-In horses and cattle, one to three drachms are the proper doses given twice in the day.

External use of the sulphate of copper is often called for as a caustic in wounds and sores growing proud flesh, which are readily controlled by it, simply by touching the parts with a piece of the crystal in powder, or in solution.

For diseases of the eye, I think it has no superior. For this purpose, three grains of the powdered sulphate to an ounce of rain water, and applied with a soft feather, or what is better, a camel's hair pencil.

For wounds which have no proud flesh in them, eight to ten grains to the ounce of water is a good and cheap application.

Copper, Acetate 0F.-This is used only for sores in the form of an ointment. Take of the acetate or verdigris one drachm, and add to it an ounce of the simple ointment. I have known sores to heal from its use, which have bid defiance almost to every other application.

Coriander.-The Seeds.-This is a useful article, and may be considered an excellent medicine for young calves having weak stomachs. The bruised seeds should be given in two drachm doses, in the milk which is given to the calf.

Creosote.-This is a peculiar smelling fluid derived from tar. Creosote has had the credit of curing glanders in man, and is a good remedy in pleuro-pneumonia in cattle, but we have better ones, and not so costly. Cases of farcy and glanders in the horse are greatly benefited by its use.

Dose.-For horses and cattle, from one to one and a half drachms, made into a mass, with flour and mo- 
lasses, and then crumbled down into some gruel, and make a drench, to be poured down the throat.

As an external remedy, it is of great advantage in mange sores, ulcers, caries of the bones, canker, thrush in the horse's feet, and foot-rot, which is so troublesome in sheep. Indeed, the more the virtues of creasote are known to farmers, raisers and breeders of stock, it will be more to their advantage. (See Prescriptions.)

Croton Oil.-A dangerous medicine when improperly used, but it is a useful medicine nevertheless, when hasty action of the bowels is wanted, as in milk fever in cows.

Dose.-For the cow ten to fifteen drops, given along with epsom or glauber salts. (See Prescriptions.)

Digitalis.-Digitalis Purpura.-The action of this powerful medicine has been much used as a sedative in lung diseases; but since we have become acquainted with the superior action of aconite, digitalis, is not now worth keeping in the veterinary Materia Medica.-Moreover, digitalis is a dangerous medicine, from the fact that it accumulates in the system, for a time, and all at once, its action is manifested by the weak action of the heart, so that in many cases, the heart ceases to beat altogether.

Doses.-For horses and cattle, twenty grains of the powdered leaves.

Disinfectants. - I have, in two portions of this book, referred to the importance of a better acquaintance on the part of farmers and stock raisers with this subject.

Volatile Disinfectants may be divided as follows:

1. Chlorine.

2. Nitrous acid fumes.

3. Sulphurous acid. 
These diffuse themselves through the air of stables and barns, and neutralize the poisonous gases which are given off from the excrement of animals, whether in health or disease.

Fixed Disinfectants.--These are such as can be mixed with the excreta and decaying matter, without destroying them for manure.

1. Perchloride of iron.

2. Permanganate of potash.

3. Gypsum-Sulphate of lime. This is not a great one, but it is useful.

4. Charcoal.-This, in fine powder, should be thrown into damp stables and barn-yards, as it will not only remove unpleasant smells, but will make good manure. Refuse tan bark, and other vegetable substances, made into charcoal, is cheap, and of great utility as a disinfectant, and adds to the quantity and value of the manure.

Fixed,-but noxious Disinfectants.-These are disinfectants which, when mixed with manure, render it valueless as such.

1. Chloride of Zinc. (Burnet's Disinfectant Fluid.)

2. Nitrate of Lead. (Ledoyer's Disinfectant.)

3. Sulphate of Copper, and Zine Solution. (Larnaudes.)

Chlorinated Soda Solution, may be mixed with manure, without destroying its qualities as such.

Chloride of Lime. Solid, or in powder, do not destroy the manuring qualities of vegetal, and other materials usually converted into manures.

Diuretics.-Medicines, which, when given to animals, increase the flow of urine, and are used in cases of swellings, and dropsies of the body, and legs of horses. 
Elecampane.-Inuta Helenium.-The root of this plant is highly thought of, and much used, by horsemen, in coughs and colds. Some make a tea of it, or decoction, and give it to the horse to drink. It is certainly a warm and grateful aromatic, and a good expectorant. 'The plant should be gathered when the seed is ripening.

Elm Bark.-Slippery. This bark, when scalded with hot water, makes a useful poultice, to irritable wounds, ulcers, and sores. A decoction of the bark will answer every purpose for which flaxseed, or linseed is used, or recommended, as in diseases of the kidneys, and bladder, produced by the use of Spanish fly, and from over-dosing with rosin, and other diuretics. In diarrhoca, in all animals, slippery elm tea, or decoction, will serve a good purpose, by sheathing the covering of the bowels, which are so apt to become irritated, and inflamed, in violent superpurgation.

Emetics.-Medicines which produce vomiting. Horses, cattle, and sheep cannot vomit, whilst the stomach remains entire. In cases of rupture of that viscus, however, vomiting is occasionally seen. Emetics are useful in diseases of the dog, and swine. Tartar emetic, or sulphate of zinc, given in from two to five grain doses, will cause $\log s$ and swine to vomit.

Epsom Salts.-Sulphate of Magnesia.-This is a valuable medicine, in diseases of cattle and sheep. No other purgative should ever be thought of, or given to either cattle or sheep. Even supposing other purgatives to be as good, none, certainly, can be so cheap, as epsom, and glauber salts, or the sulphate of soda. For all purgative purposes, the one is as good as the other. Salts should be largely diluted with water; for the quan- 
tity of fluid given with them, facilitates their operation. In domestic practice, half an ounce of salts, in one tumbler full of water, will operate as strongly as one ounce, in half the quantity of water.

Doses.-For ordinary sized cows, one to two pounds is the dose, mixed with four quarts of cold water; one ounce of ginger in powder, and the whole sweetened with molasses, or coarse sugar. For average sized calves, two to four ounces. For sheep, four to six ounces. A few drops of commercial sulphuric acid, say twenty to sixty drops, will greatly remove the naseous taste.

Ergot.-Diseased Rye. This is a curious, as well as a valuable medicine.

Use. In the calving, lambing, and foaling season, it is sometimes of great service, but should be carefully handled; as, for instance, if given to any animal about to deliver her young, and when the mouth of the womb containing the young, should not be sufficiently opened, the administration of this remedy would be attended with extreme danger, as the womb, by the powerful contraction set up, caused by the ergot, to expel its contents, the womb would likely be ruptured, or torn, and the death of the animal will follow. When the animal seems to have exhausted its strength, and the passage is open, and the young is coming in a natural way, then only should the ergot be given. If there should be a malformation in the young, or in the pelvis of the mother, it would be equally unwise to give ergot.

Doses.-For a mare, or cow, half to one ounce of the powder, is the dose. For sheep, swine, and large sized bitches, one drachm is the dose. Small bitches, ten to thirty grains. 


\section{Essential Oils.-(See Oils.)}

Ether.-Sulphuric Ether.-This is a valuable article of the veterinary materia medica, and is used in diseases of all the animals.

Use. It is used as a stimulant, and given in weakness, fevers, and in colic, as an antispasmodic. Ether is scarcely strong enough to produce anasthoesia in horses and cattle. Dose.-For horses aud cattle, one to two ounces is the dose as a stimulant, and antispasmodic. Ether should be given in cold water, to prevent its volatilization on its exposure to the air. Twice its own volume of alcohol added to it makes the spirit of sulphuric ether.

Euphorbium. $-A$ resinous juice, expressed from a cactus like vegetable which grows in Morocco.

Use. - This is only used for external purposes and is among the very few articles in veterinary medicines, capable of blistering the thick skin of the ox.

Expectorants.-This is a class of useful medicines, which cause a separation of the mucous from the throat, and air passages, thereby relieving cough and other symptoms usually attendant upon throat diseases.

Febrifuges.-Medicines capable of relieving fever.

Fenugreec.-The seeds of this annual plant, found growing in the south of Europe, is a useful cattle medicine, given in one ounce doses for loss, of appetite, ephemeral fevers, etc. It is usually, however, combined with ginger, gentian, and other medicines of that class.

Fern.-The Male.--Aspidium Felix Mas.

Use. - Given to animals affected with worms. A good remedy. 
Doses.-For the horse, one pound of the root in powder is the dose. For sheep, three to five ounces, and for the dog with tape worm, it is invaluable, destroying the worm in two hours. A purgative should be given next day.

Friar's Balsam. - This is the compound tincture of benzoin, and is an excellent article when applied to sores and wounds, as the fluid soon evarporates, and leaves a resinous covering over the wound, thus shielding it from the action of the air. (See Benzoin.)

Galbanum.-A dried juice, having medicinal properties similar to asafoetida.

Galls.-Nut Galls.-These are rough excrescences made on oak trees by holes being made in the bark by an insect.

Use.-A powerful astringent, binding the bowels of all animals affected with dysentery, and diarrhœa. And a good application to the greasy heels of horses.

Doses. For horses and cattle, powdered galls are given in from four to six drachms. For sheep and swine, thirty to sixty grains, externally applied the powdered nut may be sprinkled on the sore.

\section{Gallic Acid.-(See Tannin.}

Gamboge.-This is a gum resin from a tree, growing in the Island of Ceylon.

Use.-A useful purgative for cattle. (See Epsom Salts.)

Doses. For cattle, six to eight drachms in the dose. For sheep, thirty grains, given in solution, and should be given with other medicine of the same class. 
Gentian Root.-Gentiana Lutea.-This is an invaluable medicine in the treatment of diseases of horses and cattle, where the appetite is to be restored and kept up. In idebility, weakness, swellings of the legs and body, and where the stomach is out of order, as it is so. often.in fevers, and as a sequel to debilitating diseases, Gentian, combined with iron, is an excellent tonic, and cheap. Gentian combined with ammonia and pimenta berries, will make a horse eat almost whether he will or not.

Doses.-For horses and cattle, half to one ounce is the dose, repeating it three times in the day. For sheep, one drachm. For dogs, five to ten grains. For horses and cattle, it is best given mixed in gruel, and given in a drench out of a strong necked bottle. Gentian root should enter into all condition powders. (See Condition Powders, in Prescriptions.)

The colleges orders a tincture to be made, but the powder is all that is wanted in the treatment of horse and cattle diseases.

Ginger Zingiberis.-A well known root, and is a useful article to have in the house.

Dose.-Ginger is given in cases of gripes, or colic, whether in horses or cattle, and should enter every dose of purgative medicine, which is given to all the animals. Ginger is useful in loss of appetite in horses, and in calves an excellent medicine to give with chalk, and other things, in scours, or diarrhoea.

Doses. - For horses and cattle, one to two ounces is the dose. For sheep, two drachms. For calves of three months old, one drachm, and for younger ones less. The essence of ginger, of the drug shops, is a good way to use 
ginger, as the active principle of the ginger is given without so much inert or dead matter.

Glauber Salts.-Sulphate of Soda.-A good purgative for cattle and sheep. (See Epsom Salts.)

Glycerine.-A fluid which scarcely ever dries up, and is the saccharine principle of fats. Bower's glycerine is the best in the American market, and is inodorous, or without smell, which is the best indication of its purity.

Use.-Many sores on all the animals readily heal by the application of glycerine, especially in scratches, and sores about the heels of horses. Glycerine is a valuable remedy. In all our cities having horse rail-roads, and the authorities allow the use of salt on the streets, for the removal of snow. The slush so formed, first chills then scalds the skin of the heels, so that in a short time the skin cracks, and nasty sores are the result, and difficult to cure. To prevent this condition of things, and to protect the heels of horses from the injurious effects of the salt and slush, the application of glycerine should be made to the heels morning and mid-day, whilst the salt, slush and snow are upon the streets.

\section{Guaiacum.-This is a resin.}

Tincture of Guaiacum.-A valuable medicine in cases of rheumatism in old horses, and in rheumatic lameness in dogs.

Dose.-For horses, the dose of the tincture will be half an ounce twice in the day, given in cut feed, or in a drench with cold water. For dogs, ten to twenty drops given in a spoonful of cold water or soup.

Gum Arabic.-Gum Acacia.-This is an importa- 
tion from Alexandria, in Egypt. There are several varieties of gum.

Gum Senegal.-This is similar to the above.

Gum Tragacanth.-This is another variety of gum.

Use.-The gums are demulcents, emolient and soothing to an inflamed part, as the bowels in diarrhœa, the kidneys and bladder, when diseased and irritated.

Doses.-For horses and cattle, from one to two ounces. For calves and sheep, four drachms. Gum should be given, dissolved in warm water, and drench out of a bottle or ox horn.

Hartshorn.-This is an impure solution of ammonia. (See Ammonia.)

Hellebore.-Hellebrosus Niger.

Use.-Recommended in poll evil, by inserting a piece of the root in the fistulous opening. No dependence should be placed in it. Better agents are at hand.

Hemlock.-Conium Maculatum.-Formerly recommended in inflammation. It is of benefit in the form of a poultice to cancerous sores.

Hemp, Indian.-Cannabis Indica.-The colleges order a tincture and an extract. In the east of Europe, the resin of the plant is known as churrus, and its preparations are known as gunjoli, bang and hachish. The accounts which have reached us, I say reached us, for no such action can be produced upon man from any preparation of the hemp in use in America, as is represented to be produced amongst the subjects of His Majesty, the Sultan of Turkey. The genuine preparations of Indian Hemp produce in man great excitement and high spirits, great laughter, talkativeness, and pleasing thoughts, and a great appetite for food and sexual excitement, followed 
by sleep, from which the person recovers without any unpleasant effects. Experiments instituted by the writer with this medicine upon horses, do not warrant its introduction into the list of horse and cattle medicines.

Henbane.-Hyoscyamus Niger.-An extract and a tincture is ordered to be kept in the drug stores. Hyoscyamus is indicated in all cases where belladonna is indicated. It has, however, little effect upon animals chewing the cud. In horses, it is occasionally given to relieve cough and irritation of the throat or windpipe.

Dose. -For horses, one to two drachms of the extract is the dose, and should be given, rubbed down in a little cold water, and repeated several times in the day. Better drugs, and less expensive ones, answer a better purpose. This medicine used formerly to be relied upon for the cure of insane persons, but is now almost discarded for that purpose.

Iodine.-A chemical preparation manufactured from kelp or sea-weed, and used both rightfully and wrongfully, in many diseases. However, iodine itself is, I think, of little value as a medicine, but when united chemically with iron, copper, mercury, lead, arsenic and potassium, it certainly is one of the most useful and indispensable of medical agents, when administered internally, and applied externally.

Dose.- Iodine is given to the horse and the ox in from twenty to forty grains: for dogs, three to six grains. An ointment of iodine is made as follows:-Take one part of iodine to eight parts of lard. This is a good ointment to apply to soft swellings on the body of the horse, when applied by rubbing with the hand.

Iodide of Potassium.-Hydriodate of Potash.- 
This is a chemical union of potash with iodine, a costly remedy for horse and cattle diseases, and in most cases can be done very well without. Its action is that of an absorbent, and is, therefore, used in swelling of the glands, and other parts of the body. To get its full benefit, it should be given in syrup or molasses, so that the iodine will not be lost, leaving the water only behind.

Dose.-From twenty to thirty grains for horses and cattle, repeated three times in the day. For the dog, five grains.

(1.) Iodide of Arsenic.-Highly recommended by some persons for glanders, farcy and purpura in the horse.

Dose.-Five grains given once a day, in cut or mixed feed.

(2.) Iodide of COPPER.-A valuable remedy, but costly. It should be used only for valuable horses, in cases of debility and loss of condition, mixed in cut or mixed feed.

Dose.-From one to two drachms, given twice or three times in the day.

(3.) IodIde of Iron.-This is even more valuable than that of the iodide of copper, and is given for the same purpose, and in the same doses. To use the iodide of iron once, it will be used again. Its high price is the only drawback. Iodide of iron enters into the powders and balls which are given to the English race horses, whilst in the trainer's hands; - a high recommendation.

(4.) Iodide of Sulphur. - A valuable remedy in mange, and other skin diseases, and may be given internally also, in the same disease.

Dose.-For horses, give two to four drachms in the 
animal's feed. For mangey dogs, give ten to fifteen grains once a day, for both horse and dog; give it at night, as warmth assists its action very much.

(5.) Iodide of LEAD.-Too weak for veterinary practice, but excellent for swellings on the body of man. It is only used externally. Iodide of lead is of a lovely, yellow color.

(6.) Iodide of Mercury.-Bin-Iodide of MercuryRed Iodide of Mercury, etc.-This preparation of iodine is a medicine that the educated veterinary surgeon cannot do without in the practice of his profession; for there is nothing which will so well answer his desires and his purposes, as the iodicle of mercury. Iodide of mercury is not used internally, in any disease, as calomel contains the same internal action as that of the iodide.

Use.-Iodide of mercury is used in all cases of induration and swelling of the glands of the neck, tumors, whether of bone or soft tissue, splints, spavins, ringbones, wind galls, shoulder-joint lameness, thoroughpin, and will answer well for an ordinary blister, for whatever purpose. The only objection to it as a blister over an extensive surface, is its painful operation.

The iodide of mercury is used as an ointment; one part of the red iodide of mercury to eight parts of hog's lard, well mixed together. This ointment is to be well rubbed into the parts to be cured; swelling will follow its action, but will subside in a few days, if one application be enough, which will be known, if the enlargement has been broken or has disàppeared. If not, apply in from five to six days again, scarcely rubbing so hard as at the first application, as the skin is more easily acted upon at this time. But whether one, two or half a dozen applications be necessary, let sufficient time elapse before 
each succeeding application, so as not to prevent the hair growing, and thereby blemish the part. Apply lard once a day between each application, and occasionally wash the parts with water, not too warm, and lard, or oil the part when dry. The horses' head should be tied up, so that he cannot get at the parts with his mouth. A few hours will be long enough. Place plenty of soft bedding under the feet, so that, by stamping, the horse will not break or hurt his feet.

Observe.-When, apparently, the tumor, gland, or other enlargement, does not disappear, or go away at once, surprise is sometimes expressed, to see it totally go away, as if of its own accord. Thus the red iodide of mercury exercises a powerful influence, long after its use has been dispensed with. Iodide of mercury, occupies, in my estimation, in external diseases, what the preparations of aconite does in internal affections, whether in horses or cattle.

Several other preperations of iodine are made by the manufacturing chemists; such as the iodide of gold, and silver, which are of no use in horse and cattle diseases.

Ipecacuanha.-Cephaelis Ipecacuanha.-In veterinary practice, ipecacuanha is only used for dogs, in fifteen to thirty grains, as an emetic, when first attacked with distemper.

Iron.-Ferrum.-This is one of the most valuable medicines we have to recommend, in many of the most important diseases, of all the animals. This will be readily seen, when it is stated that iron is a constituent of the blood of all warm blooded animals, and without iron, being in proper quantity, in the blood of an animal, it cannot be healthy-is anxmic, commonly called impoverished blood. In fact, iron is an elimentary principle, essential to health.! 
Metalic iron is rarely used in veterinary practice, except it be in the form of iron filings, given by some persons, in cases of worms in horses. The sulphate is just as good for this purpose. Iron filings, called Ferrum Redactum, is much used in the weakly and sickly female. To prevent the filings from oxidizing, or rusting, the filings are put into a tube, similar to a gun barrel, at a strong heat, and the filings are plunged into cold water, which give them a sky-blue color; the finer the blue, the better the medicine. For the valuable dog, iron-filings, in this form, may le given, in five to ten grain to a dose. The following preparations of iron will be used for the horse and ox.

(1.) Carbonate of Iron.-Ferri Carbonas.-This preparation of iron, is only used in veterinary practice, for the dog, on account of its mildness.

(2.) Sesquioxide of Iron.-Rust of Iron.-FeRUGO. This form of iron is only used by veterinary surgeons, in poisoning from arsenic, as an antidote, by forming in the stomach, an insoluable arsenite of the protoxide of iron.

(3.) Sulphate of Iron.-Ferri Sulphas.-GreeN VITRIOL-Copperas.-This is one of the best, and cheapest preparations of this valuable metal, that can be used in diseases of horses and cattle.

Use.-In medicinal doses, the sulphate of iron is tonic, and astringent; therefore, it is used in cases of weakness, want of condition, looseness of the bowels, swellings of the legs, body, sheath, breast, etc., from its improving, and adding richness to the blood, and giving tonicity and health, to the fibrous serous tissue, from which the fluid which causes the swelling arises. In pleuro-pneumonia in cattle, after fever has abated, nothing will restore and prevent effusions of serum, or fluid, in the chest, like 
sulphate of iron. Effusions in the chest of cows, and neat cattle, is the cause of so many deaths, from cattle disease. The effusions form connecting links between the lungs and the sides, from which adhesions take place in that disease, and from which the animal can never be restored to good health, although the beast may live for a year or two. How important, then, to know of a medicine which offers so much hope!

In addition to the diseases just named, sulphate of iron is the medicine to be used in all cases, where the powers of life are low and depressed. In red water in cattle, bleeding internally in all animals, dysentery, purpura, scarlatina, and in debilitating diseases generally, no medicine offers so much as the sulphate of iron.

Observe.-Sulphate of iron should not be given while inflammation and fever lasts; it is time enough to give it after all irritation has subsided. Sulphate of iron should always be combined with a vegetable tonic, such as gentian, or ginger. Iron changes the dung to a green color, as if the animal was at pasture.

Doses.-For horses and cattle, the dose is two to three drachms, with the same quantity of powdered gentian, two to three times in the day, to be given, mixed in a quantity of cold water; and drench out of a bottle, if the animal does not eat it readily, mix with cut, or soft feed.

(4.) Perchloride of Iron.-Whether in a fluid or solid state, this preparation is valuable for applying to bleeding wounds, to stop the flow of blood, which it will readily do, if properly applied, and the blood vessel is not too large, that is wounded. It should be applied with a soft brush, or a pleget of soft cloth or cotton, dipped in it, and laid over the wound, and kept bandaged. Perchloride of iron should be kept in every farm, or 
country house, for this purpose. It should be kept in a glass, stoppered-bottle to keep it pure. (See Iodide of Iron.)

Jalap.-Convulvulus Jalapœ.-This root derives its name from a town in Mexico, callę Xalapa, and grows fully six thousand feet above the sea level. This medicine is in veterinary practice used only as a purge for the $d o g$, in from twenty to sixty grains.

Juniper Berries.-Juniperis Communis.-The Fruit.-This medicine is valuable in horse and cattle diseases, as a stimulant to the stomach, in loss of appetite; and in convalescence, from debilitating diseases.

Dose.-For horses and cattle, one to two ounces is a dose. Dogs, twenty to forty grains.

Kino.-A juice of several plants. This is used as an astringent in diarrhœa in all animals, and is considered more powerful than catechu, (which see.)

Kousso.-The flowers of this plantare brought from Abyssinia.

Use.-To expel, or destroy tape worm in man, and the dog, and is prepared and used in the following manner. Take of kousso half an ounce, of warm water half a pint, let it stand till cool, and give it, flours and all, and give next day a dose of castor oil.

Laudanum.-(See Opium.)

Laxative.-A mild purge.

Lead.-Plumbum.-Metallic lead in the form of shot, is used by our horse dealers, to relieve temporarily the heaving, or symptoms of broken wind, or heaves in horses. It will be needless for me to say, that if the lead is not 
speedily converted into an insoluable oxide, the animals so treated will die in a month or two.-(See Lead Poisoning.)

(1.) OXIDE OF LEAD.-This preparation of lead is used in the manufacture of common sticking plaster, or Diachylon.

(2.) Iodide of Lead.-(See Iodine.)

(3.) Acetate of Lead.-Sugar of Lead.-Solution of acetate of lead is known by the name of Goulard's Extract of Lead, and was formerly, and is still by some persons recommended, and used in cases of sprains, and as a wash for diseased eyes. Better, and less injurious agents are now used by the educated veterinary surgeon.

Observe.-Goulard's Extract, or lead water, should never be used in diseases of the eye, as it can do no good beyond what cold water can do, but it dulls the eye, by making it hazy and opaque. It will be well to remember this, and act upon it, not minding what old and antiquated books, and individuals may say, or think, in regard to what is here recommended.

Lime.-Oxide of Calcium (which see.)

Carbonate of Lime.-(See Chalk.)

Linseed.-Linum Usitatissimum.-Ground flaxseed makes the best poultice, as it is less irritable, and retains its moisture better than most articles in use for that purpose.

Cake Meal.-That portion which is kept after the oil has been expressed from the seed, is a good feed for horses and cows, given occasionally, and makes a good and much cheaper poultice than the most costly seed.

Linseed Oil._Oleum Lini._This is a good and safe 
purgative for the horse, and should be given by farmers and non professional persons in preference to any other article. The English veterinary surgeons use linseed oil for colic in the horse, in the following combination : Linseed oil, one pint, and two ounces each, of oil of turpentine and laudanum. In cases of choking, in either horses, or cattle, a half pint of linseed oil should be poured down the throat, so that by its emollient properties the substance may pass readily down the gullet.

Doses.-For horses, one to two pints is the dose used for a purgative. For scalds and burns, linseed oil is mixed with lime water. (See Lime Water.)

Liquorice Root.-Glycyrrhiza Radix.-This was formerly in use for making balls for horses, but it is now superseded by molasses.

Lobelia Inflata.-Indian Tobacco.-This medicine is in great use by the eclectic physicians in the United States as an emetic, which fact, I believe, has induced Dr. Dadd to recommend it to veterinary surgeons and horsemen of this country. Does the Doctor not yet know that the horse, the ox and the sheep, cannot vomit? Therefore, it is not entitled to such a place in the veterinary Materia Medica.

Logwood.-Hcematoxylon Campechianum.-This is a valuable medicine and not well known, and consequently not appreciated.

Use.--One of the very best astringents for binding the bowels in diarrhoa and dysentery, in all animals, especially when accompanied with irritation of the bowels. Logwood is cheap, and sold in chips, and is prepared and given in the following manner : 
Logwood Chips two ounces, boiling water one pint, allowing it to stand till cold, then strain through cloth, or a fine sieve.

Dose.-For horses and cows, this quantity will make one dose. For calves with scours, one to three ounces will be the dose, and repeating it if the case demands it. A safe remedy, and cheap.

\section{Lunar Caustic.-(See Silver.)}

Lupuline.-The Hop.-Makes a good poultice, applied to the udders of cows, when lumpy, hard, and for hastening the suppurative process.

\section{Lytta-Cantharides.-(See Spanish Fly.)}

Magnesia-Oxide of Magnesium.-Used sometimes in young foals and calves, when they have no appetite. A good antidote for arsenical poisoning.

Dose.-For these animals, two drachms to six is the dose, and a little ginger should be added to it to prevent griping.

(1.) Carbonate of Magnesia-Magnesia Alba.-The action of this is similar to the preceding.

(2.) Sulphate of Magnesia.-(See Epsom Salts.)

Manganese.-A metal used as a disinfectant, in combination with some one of the mineral acids. For the way to use it, (See Disinfectants.)

Marsh Mallow.-The Root of Althea Officinatis. Used in the form of an ointment, but not of much utility. 


\section{MER̃CURY.}

It however still holds a place in horse and cattle medicine. It can be done withont.

Marigold-Calendula Officinalis.-A garden plant. Tincture of. This is a good application to sores, ulcers and abscesses, and is applied with soft cloths, saturated or moistened with it, and laid over the affected part. It is a new remedy in burns and scalds.

Mercury.-A liquid metal called quicksilver. In its metalic state it is not used as a medicine. There however is a prevalent idea in the minds of ignorant persons, that doctors, and veterinary surgeons give this substance to force a passage through the bowels, and that if it fails the bowels and stomach will be ruptured, or torn. If the patient should die, the blame is not unfrequently laid on the use of quicksilver by the doctor, when this substance was never thought of for any such purpose. Quicksilver has no action whatever on the animal system, either in health or sickness.

(1.) Mercury With Chalk-Gray Powder.-Used in diarrhoea in calves, in doses from ten to fifteen grains, given with a little ginger, and mixed with wheat flour gruel.

(2.) Iodide of Mercury.-(See Iodine.)

(3.) Bi-Chloride of Mercury-Corrosive Sublimate. A dangerous poison, and should never be given to any animal. It is however used in solution, in some skin diseases, as in ring-worm and mange. When so used, only a small portion of the body should be washed with it one day. For this purpose, four grains of the sublimate to two ounces of rain water. For dog, two grains to the ounce, in water, will be strong enough. 
(3.) Nitrate of Mercury-Ointment of.-This is the citron or golden ointment, and is a good remedy in ring-worm. Unfortunately, however, it spoils with long keeping, and is not made extemporaneously, therefore it is often rancid, and of little value.

(5.) Oxide of Mercury-Red Precipitate.-Used in the treatment of unhealthy ulcers and sores, in the form of powder, ointment, and is the principle in the yellow wash already spoken of, when speaking on the subject of lime. (Which See.)

(6.) Sulphate of Mercury-Turbith Mineral.-A medicine of no use, and should be expunged from the books on horse and cattle diseases.

(7.) Sub-Chloride of Mercury-Calomel. - A medicine I never use, and will not recommend it for internal administration. In the form of the black wash, and sprinkled upon sores, is a good remedy. Calomel is recommended for thrush in the feet of horses, by inserting it into the cleft of the frog.

Mercurial Ointment.-This is sold in the drug stores.

The ointment of mercury is chiefly used in skin diseases, as mange in horses and dogs. But a better remedy will be found in sulphur, and its preparations, which are not only more safe, but more certain of curing the case.

Mezeron-Daphne Mezerum.-A plant, the bark of which an extract is made, and from the extract an ointment is made, one drachm to four parts of lard, and is used for the purpose of keeping up the irritation of blisters.

Morphia.-(See Opium.)

Muriatic Acid.-(See Acids.) 
Mustard Seed.-Sinapis Nigra.-Used as an irritant on parts of the body, where the Spanish fly would be improper and dangerous. The belly, and over the loins are the usual places where mustard is applied. In lumbago, and sprains of the back and loins, and in pain in the bowels, mustard is useful. The mode of application of mustard is as follows: If the hair of the part be long, cut it off, then foment the part with warm water, and immediately rub into the parts a handful of the best flour of mustard. This is more effectual than laying a paste or poultice without rubbing.

Volatile Oil of mustard makes a good counter-irritant, when a few drops are rubbed into the skin. Vinegar added to mustard does not as it is "thought produce a better effect. Warm water answers every purpose.

Myrrh.-A gum resin, used in the form of a tincture and a compound tincture, to sores, and a good application in sore mouth, from the bit, or other cause.

Narcotics are medicines which act upon the brain, and thereby allay pain. Example, opium and its preparations, aconite, and chloroform.

Neat's Foot Oil.-Used for skin diseases, and for allaying the irritation of blisters, and keeping the skin from cracking.

Nervines.-Medicines relieving pain, without producing narcotism.

\section{Nitric Acid.-(See Acids.)}

Nitre-Saltpetre.-This medicine has long been used as a diuretic, and as a febrifuge in low fevers; cheaper and better medicines are daily in use for this purpose. Nitre is not a cheap medicine, nor yet is it an indispen- 
sable one. The days are past when emetic, tartar, nitre and digitalis were the remedies used in all cases of inflammation, irritation and fever. This formula has deceived many a man, and has been the means whereby many thousands of valuable animals have been lost by trifling with remedies in use half a century ago. Wherever nitre is indicated, I use the sulphite of soda, and I have no cause to regret the change. A bundle of fresh cut grass given to a horse, will have a better, and more soothing effect than nitre; when given in any disease, nitre should be left to fulfil its destiny by making gunpowder to liberate the toiling millions of other lands from the hands of the despot and the oppressor.

Nux Vomica.-Strychnos Nux Vomica.-An active poison in large doses, but a valuable agent in the cure of diseases in all animals. The powdered nut is uncertain in its effects, therefore the tincture, and the alkaloid should only be used.

Use.-A nervine, and used when the nerves are depressed and weak, just as aconite is used when the nerves are strong and excited. The one medicine is used in depression, and the other in diseases with exalted symptoms. Paralysis or palsy is the loss of power in the motor nerves of the part affected. Therefore, nux vomica is indicated, in twitching of the muscles of all animals, and in glass eye, in the horse depending upon the want of nervous energy in the optic, or nerve of vision.

Tincture-Doses of.-For horses and cattle, the dose is from ten to twenty drops, repeated three to four times in the day.

Strychnine-Dose.-For horses and cattle, the dose is one grain, given once a day, gradually increasing the dose till three grains are given in the day. To get full 
benefit from it, it will have to be given for a week or two, if the beast has not got well by that time. In the use of strychnine, care and good judgment must be exercised, for it must be remembered that however useful a drug, or medicine may be, is no argument that it can not be abused. Strychnine should be administered in feed, if the animal will eat it, if not give it in gruel in the form of a drench. Twelve grains is the dose required to kill a full grown horse.

Arsenite of Strychnia is recommended by the French veterinary surgeons, in nasal discharges, but with what effect is not stated. If it does not cure, as I suppose it does not, it only serves to show to my mind how utterly ignorant veterinary surgeons are in regard to the true pathalogy of those discharges, supposed to be glanders. Mr. Gamgee, on the International Veterinary Congress, held at Hamburg, says when the subject of glanders was mentioned, no one responded. Why? certainly not that they knew all about it, no, the contrary is the case, for they knew nothing at all about its nature, and hence we have the French using a drug the least of all likely to change, arrest, or cure the disease. Prussian blue is said to contain ten per cent of strychnia, some packages contain as high as sixty grains.

Oak Bark.-Quercus Cortex.-This is a good astringent for outward use, or to sores which discharge a good deal. The bark is boiled, half an ounce to a pint of water. This decoction is an excellent remedy for drying up the moisture of greasy heels, so troublesome in horses. In diarrhœe in calves, given in four drachm doses, much good will follow its use.

Oils.-There are two varieties of oils used in medicine-fixed and volatile. 
Fixed Orls.-Castor, olive, linseed, croton and neats'foot oil. The uses of these oils will be found treated of under their respective heads.

Opium.-Papaver Somniferum.-The dried juice of the white poppy, and is one of the most remarkable substances in nature. There are several varieties of opium: Turkey, Egyptian, East Indian, Persian, and European opium. The medical preparations of opium are several:

Morphia, or morphine, is the most important preparation of opium, and sold in the drug stores in the form of white crystals, and in the form of a liquid;-liquor morphia acetas, and liquor and liquor morphia sulphas. Either of these preparations of opium are much better medicines than the crude opium itself. Each fluid ounce contains one grain of the morphine, or the true principle of the opium, and one grain of morphine is equal to three grains of opium, or to forty-five drops of the tincture of opium, commonly called laudanum.

$U$ ses.-Opium is a narcotic, or reliever of pain, and is especially recommended in milk fever in cows.

Dose.-The dose of the acetate, sulphate, or muriate in crystals, will be for the horse from twenty to forty grains. For the cow with milk fever, the dose will be from forty to eighty grains. To a medical man, such large doses may seem to him enormous, for in man the dose is from half to one grain.

Horses will scarcely show the least effects from the administration of from two to four drachms of the powdered opium. On cattle, opium has even much less power than it has on horses. Cows can take one ounce, and sheep half a drachm of powdered opium, without suffering. The doses of crude, or powdered opium for horses, will be from one to two drachms, and for cattle, two to four 
drachms. In veterinary practice, opium is not now so much used, as aconite answers almost every purpose for which opium was given, and without in any way binding the bowels. But in case of milk fever, opium, or its alkaloid morphia, is well worthy of a trial, and if the case be taken in time, it will scarcely deceive.

Laudanum.-Tincture of Opium.-Every fifteen drops of the tincture contain one grain of opium. Laudanum is the most costly of all the preparations of opium; and not only that, the large quantity of alcohol, or spirits of wine it contains, is a decided objection to its use in many diseases. Crude opium rubbed down with a little water will be far better when it is to be used at once, or not to be kept for any time. Laudanum is used in lotions and liniments for the relief of pain, and it enters into eye washes, for the same purpose.

Wine of opium contains spices. Not much used.

Codia, narcotine, narceine, paramorphia, papaverine, meconine and meconic acid, are the various constituents of opium, but which are not used in medicine, either in domestic or veterinary.

Battley's sedative drops, so well and familiarly known throughout the world, wherever the English language is spoken, though a secret, are known to contain opium, water and a little spirit, and is one-third stronger than laudanum.

Pariera Brava. - A root which is to be sliced, and add one pint of water to one ounce of the root. Boil, and strain when cold. This is a good tonic for horses. Mix with the feed, half a pint to the dose.

Peach Leaves.-One ounce infused in the same way as tea, with one pint of water. This infusion is an ex- 
cellent application to the skin of animals having the itch and skin diseases.

Peppermint.-Mentha Piperita.-The essence of this garden plant is sometimes given to horses having colic, and given in doses of twenty drops. Dogs can have. three drops for a dose, in the same disease.

\section{Pepper.}

(1.) Black Pepper.-Piper Nigrum.-Occasionally given to horses affected with colic.

(2.) Jamaica Pepper.-Pimento-Allspice.-This is a valuable medicine for horses and cattle affected with fever, loss of appetite and indigestion.

Doses. - From three to five drachms, and is given in combination with ammonia.

(3.) Capsicum.-Cayenne Pepper.-This is also used for the same purpose as the above, and given in from twenty to thirty grain doses.

Pepsin.-The active principle of the gastric juice of animals. Its cost prevents its use in calves having diarrhoea, when the discharges are like milk. A good substitute for pepsin is rennet, which farmers should have about them; not only for cheese-making purposes, but for use in looseness, or scours in calves. It assists the stomach to digest the milk, which, in diarrhœea, lays upon the stomach, without being digested or changed. Hence the white diarrhœa, so often seen in calves.

Petroleum.-Rock Oil.-This substance was formerly highly recommended in chest diseases, but it has been succeeded by more certain and successful drugs for this purpose. However, it is still occasionally used as an external application for sores, and for the destruction 
of lice, etc., in the skin. It is apt to leave a blemish by causing the hair to fall off, and in some cases where I have seen it used too extensively, perhaps the hair did not come again. The better way to use coal oil is to mix equal parts with some other oil having no acrid principle.

Phosphorus.-An elementary substance resembling wax. There is, however, another resembling brick dust, when in a mass. The preparations of phosphorus are numerous:-Hypophosphate of ammonia, hypophosphate of iron, and the phosphate of lime, or bone, or earth, phosphate of soda, and of quinine. Phosphoric acid, diluted, is the one which should be used in veterinary practice.

Phosphoric Acid-Is used in diseases of the bones of young colts.

Doses.-For young colts, the dose will vary from ten to sixty drops, given diluted in cold water.

Pitch.-Burgundy Pitch.-The concrete juice of the Abies excelsa.-This is used in the composition of the plaster, formerly used over the loins, in weakness, sprains, lumbago, etc., in the horse.-Not now recommended.

Pomegranate Punica.-Granatum.-The bark of the root of this tree is used for the destruction of tape worm, in all animals. Take of the bark, one ounce; water, one pint; and boil down one half. The dose for the dog will be a small wine glassful.

\section{Poppies.-(See Opium.)}

Potash.-Oxide of Potassium.-Preparations of potash are many:-

(1.) Caustic Potash.-Used as its name indicates, as a caustic, and is a favorite one of mine. Many persons 
object to it, on account of its extreme fluidity, which I consider to be its most valuable property; as if it did not readily assume a fluid form, its caustic properties would not be so good. Indeed, according to its fluidity, when applied to a part, so does its caustic property entirely depend. For the quick reduction of proud flesh, it has no superior.

(2.) Carbonate of Potash.-This is sometimes used as an antacid, but is not much used in horse and cattle diseases.

(3.) Sulphuret of Potash.-Hepar Sulph.-Liver of Sulphur.-This is a valuable remedy when applied to mange in all animals. Dissolve an ounce in a pint of water, first having rubbed the mangey part with fine sand, to expose the insect to the action of the sulphuret.

(4.) Sulphate of Potash.-(See Glauber's Salts.)

(5.) Iodide of Potash.-(See Iodine.)

(6.) Nitrate of Potash.-(See Saltpetre.)-The acetate, and tartrate of potash, are not used in veterinary practice.

(7.) Chlorate of Potash.-Used for the same purposes as the nitrate, and in the same doses. The same may be said of the permanganate.

\section{Prussic Acid.-(See Acid.)}

Pumpkin Seeds.-An excellent remedy for destroying tape worm.

Purgatives.-Medicines which empty the bowels.

Pyroxylic Spirit.-Wood Naphtha.-Used to relieve chronic cough, and is given in half-ounce doses in gruel.

Quinine.-(See Cinchona.)

Rennet.-(See Pepsin.) 
Rhubarb.-Rheum Palmatum.-Rhubarb is tonic and purgative in dogs, and other carnivorous animals, but in horses and cattle it has scarcely any effect whatever, further than improving the appetite. Better, and much more efficient and cheaper drugs are used in horse and cattle diseases. I know of a gentleman farmer, who uses the spiced rhubarb in young calves, in cases of looseness of the bowels, and in diarrhoea. The dose of the spiced rhubarb, will be from one to two teaspoonfuls, repeating it two to three times a day.

\section{Ricinus Communis.-(See Castor Oil.)}

Rochelle Salts.-(A Tartrate of Potash and Soda.)

\section{Saffron.-Crocus Sativus.-(See Colchicum.)}

Sagapenum.-This is a gum, and is used for the same purposes as asafoetida.

Sal Ammonia.-Chloride of Ammonia.-(See Ammonia.)

Salt.-Z'able Salt._Chloride of Sodium, a valuable condiment, when given with the food of animals. Salt is a laxative in the horse, cattle and sheep, and is considered a preventive of sheep rot.

Sal Volatile.-This is the compound spirit of ammonia.-(See Ammonia.)

Sassafras.-Laurus Sassafras.-This is a favorite remedy with farmers for their horses, and is given to them in the spring of the year, to strengthen and improve the appetite. Sassafras may be given to horses, either in the form of a powder, or as a decoction, or tea, and mixed with the food.

Sarracenia Purpura.-Indian Cup or Pitcher Plant.-This plant is found growing along the coast of 
Labrador, to the shores of the Gulf of Mexico, on wet and marshy land.

Use.-This plant has recently proved to be one of the most useful, in the whole list of medicines, and from all accounts, it is, and will still be a blessing to thousands who may become affected with small pox. For small pox in cattle and sheep, it is not only a sure remedy, but also, as good a preventive for the disease. The form and manner of using this medicine, is in that of a tea. The time, however, will soon be, when the chemist will provide us with an extract, or an alkaloid of this plant, which will enable us to give the essence of the plant without any inertmatter. Take from one to two ounces of the dried root, and slice in thin pieces, place in an earthen pot, or other vessel, and add a quart of cold water, and allow the liquid "to simmer gently over a slow fire, for two to three hours, so as to lose one fourth of the quantity.

Dose.-Give to cattle, half a pint; and to sheep, two wine glassfuls; repeating the dose in six hours, when a cure will generally be effected.

Savin.-Juniperus Sabina.-The oil of juniper is used in veterinary practice, for the purpose of destroying worms in all animals.

Doses.-For horses and cattle, the dose is from three to four drachms, and for the dog, three to five drops. An ointment of the dried savin tops is used for the purpose of keeping up the action of blisters. One part of the fresh tops, to sixteen parts of lard.

Sedatives.-Medicines which allay inordinate action of the heart.

Senaga Polygala Senaga.-Snake Root.-This is like other of our native herbs, -is often used by country folks in the spring of the year, by giving a 
decoction, or tea of the snake root to their horses. It is an excellent remedy in coughs, bronchitis, cold, etc. Take one ounce, and boil in a pint and a half of water, and strain through a sieve or strainer.

Dose.-For a horse, half a pint; mixed in his feed, night and morning.

Sialagogues.-Medicines which increase the secretion of saliva, such as aconite in large doses, and second crop clover, after it has been exposed to cold nights or a little frost.

Silver.-Nitrate of-Lunar Caustic.-Used as a caustic to unhealthy sores and ulcers, and a solution, three to four grains to an ounce of rain or distilled water, is used to the eye in purulent ophthalmia. The nitrate of silver is too costly for general use. The sulphate of copper, or blue stone, will for most purposes answer as well, and is very cheap.

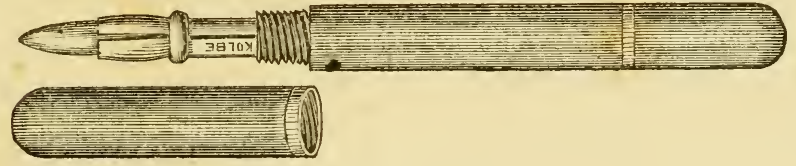

CAUSTIC HOLDER.

Soap.-A combination of fatty acid with an alkali.

Soap Liniment.-Opodeldoc.-A very useful application for sprains, bruises, and for stiff joints.

Soda.-A mineral alkali.

(1.) Carbonate of Soda.-Useful as an antacid, in doses of from two to four drachms.

(2.) Sulphate of Soda.-(See Glauber's Salts.)

(3.) Sulphite of SodA.-This is made by passing a stream of sulphurous acid through a concentrated solution 
of carbonate of soda. This is a valuable article in the treatment of diseases of horses and cattle. The sulphite of soda possesses the power of neutralizing the action of zumins, ferments, or leavens, when introduced into the circulation. Professor Polli, of Milan, has clearly demonstrated, that this substance is capable of purifying the blood of noxious matters, and further, he has by his experiments upon dogs, proved that glanders can be cured by it, for he says, that forty-five grains of the virus of glanders taken from the nares of the nose of a glandered horse, and injected into the circulation of a dog, after the disease had shown itself in the dog, it was readily cured by the administration of the sulphite of soda. Upwards of seventy cases have thus been experimented upon by this distinguished professor. All going to show the value of soda, as a purifier of the blood, by its alterative effects.

Sulphite of soda is indicated in all cases of eruptions on the skin, in farcy, glanders and purpura in the horse, and in pleuro-pneumonia and rinderpest, etc. in cattle. Wherever pus is thrown out as a product of disease, the sulphite of soda should be given. Dogs with distemper should have it to keep the circulation pure of pus. The blood of the dog becomes contaminated in this way, and hence, the good dog falls a victim to the scourge of fine bred animals.

Dose.-For horses and cattle, the dose is from half an ounce, to one ounce; given twice in the day. For dogs, twenty grains given in camphor water. In farcy and glander or other diseases, accompanied with debility, the soda should be combined with gentian and other tonics.

Spanish Fly.-Lytta Cantharides.-An insect ground into powder, and mixed with lard or oil, make what is familiarly known as a blister. The Spanish fly is, besides its blistering qualities, a very valuable internal medicine, 
a powerful-tonic and altrative, and has been used with success, in the hands of Mr. Vines, of London, England, in the cure of farcy and glanders in the horse. In the same diseases, and where the lungs were free from tubercles, I have cured them with the Spanish fly, gentian, copper, and the sulphite of soda.

Dose.-Spanish fly is given to the horse in five grain doses once a day only, and mixed in cut or soft feed. Horses are more easily affected by the Spanish fly than mares. Blisters of Spanish fly are made as follows: Powdered Spanish fly, one drachm; hog's lard, six drachms; mix, and apply, by rubbing with the hand into the part to be blistered, wash off the part the next day, and oil or lard the part for a week, and the swelling will leave when the irritation has gone out of the part.

The tincture of Spanish fly was formerly used as the basis of the old sweating blister. An excellent application for the removal of a splint, or soft tumours about the legs of horses, is made as follows: Tincture of Spanish fly, one ounce; oil of croton, twenty drops; well rubbed into the part, it acts like magic; that is, if the tumor can be removed at all by any means.

Specifics.-Medicines which cure disease, but the manner of action is unknown.

Spermaceti.-This is a solid crystalline fat, found in the large head of the sperm whale. There is a plan, however, by which it is said that spermaceti can be made out of the muscles of a dead cow or horse, by enclosing the flesh in a box perforated with holes, and sinking it in a running stream of water for a month or more, when the flesh will be converted into a mass of tough, solid matter. After which, it is treated with nitrous acid, poured over it to remove the offensive smell, and to separate the fat. 
Another plan is, to pour nitrous acid over the flesh without immersion in the brook, and which is said to convert the flesh in the short period of three days, into a yellow colored spermaceti. Another plan is mentioned by Lord Bacon, in his work Sylva Sylvarium, that the flesh of an animal may be changed into a fatty substance, by cutting it into pieces, and put into a glass vessel, and covered with parchment, and allowed to stand for six to seven hours in boiling water. Experiments of this kind might result in profit to those who might undertake them. The use of spermaceti in horse and cattle diseases, is for the making of ointments and salves, especially in hot weather, when lard cannot be conveniently carried without the risk, not only of losing a portion of the ointment, but of spoiling the clothing, or other articles coming in contact with it.

Spirits of Nitrous Ether.- - Sweet spirits of nitre is well known to most persons as a good household remedy for fevers, etc. In the treatment of the discases of horses and cattle, sweet spirits of nitre is used as a stimulant and antispasmodic, or to a horse having a chill, and in colic. For colic, it was formerly used to be given 'in combination with laudanum.

Dose.-For horses and cattle, the dose of sweet spirits of nitre will be from one to two ounces, given in cold water to prevent loss.

Spirits of Wine.-Alcahol.-This is used for making tinctures from the various plants in use for medicine. It is also a good stimulant; much better than the bad whisky which is so often poured down the throats of horses affected with colic.

Sponge. $-\Lambda$ valuable article for the cleansing of wounds and sores.

Starch. - A good remedy in diarrhoca in all animals, 
combined with a few grains of opium, and a little ground ginger, or a few drops of the tincture, commonly called the essence.

Styptics.-Substances having the quality of stopping bleedings from wounds. Examples:-perchloride of iron, cobwebs, the hot iron, and the bandage.

Sudorifics.-Medicines which produce sweating.

Sugar of Milk.-Much used by homœpathists, both domestic and veterinary, in the form of powder, but oftener in the form of globules or small pellets. Not recommended for the treatment of any disease. Molasses is the best form in which to give sugar to animals, and is useful in cases of sick cows, mixed in a drench.

Sugar of Lead.-(See Acetate of Lead.)

Sulphuric Acid.-(See Acids.)

Sulphur-Brimstone.-Sulphur is kept in the drugg stores in three forms, flower of sulphur, milk of sulphur, and sulphur vivum.

Use.-Sulphur is chiefly used for skin diseases, in the form of an ointment. Sulphur is believed by many persons to be a great preventive of diseases and distempers in all animals, and is one of the ingredients of an incongruous mass extolled and recommended by the Hon. Isaac Newton, Commissioner of Agriculture, as a preventive of cattle discases. Whatever may be the medicinal virtue possessed by sulphur, it certainly has no prophylactic offects mixed with tar, etc. The simple ointment of sulphur is made as follows: take one part of the flower, or sulphur vivum, and mix with four parts of lard. In winter, when lard is hard, oil should be used instead, thus forming a liniment of sulphur, and is more easy of application.

Doses.-Sulphur as a laxative for horses and cattle, should be given in doses from one to three ounces, and 
administered in gruel, in the form of a drench. For dogs, the dose is one to two drachms. Not recommended as an internal medicine.

Sulphurous Acid Gas.-This is one of the most powerful disinfectants we have. The great objection to its general use for this purpose is, its poisonous character when breathed or inhaled to any extent. But from recent experiments instituted by Dr. James Dewor, of Kirkcaldy, Scotland, for testing the efficacy of sulphurous acid gas as a disinfectant, results are shown which lead to the conviction, that the diseases such as cholera, in man, and rinderpest and pleuro-pneumonia in cattle, may not only be prevented, but much modified by this, hitherto, considered poisonous gas. The method of generating sulphurous acid gas is very simple and inexpensive. It is only necessary to have a small chaffer of red hot cinders from a coal fire, set a small crucible on the hot cinders, and place a piece of sulphur-stick about as large as a man's thumb into it. This will fumigate a large cattle shed, or stable, in twenty minutes. Contrary to expectation, the animals seem to enjoy it, and at the same time it acts as a tonic on man and beast. The shed or other house must be well ventilated, by having the windows a little open during, after, and before the fumigation. Sanitary rules must be enforced in regard to cleanliness, removal of dung-heaps, etc. During the prevalence of such epizootics as are above named, the fumigation may be made according to the foregoing directions, four or five times in the day. It is further said, that the treatment has not only cured some cases of the above named diseases, but mange, ring-worm and lice have also vanished before it, and that greasy heels in horses have also been cured by it, while severe cases of tubercles of the lungs, glanders, and farcy have 
also been relieved by the inhalation of this gas. This is well worthy of a fair trial, and if it but accomplish half what is claimed for it, a great and cheap fumigation and disinfectant is now within our reach. But, bear in mind still, that it is a poisonous gas, and to prevent this effect, have free ventilation through the whole place.

Sulphate of Zinc.-White Vitriol.-This is a valuable article for healing wounds and sores. Not administered internally to any animal, except as an emetic to dogs. As an external wash for sores, one part of zinc to twenty of rain water, will answer for most purposes. For eye wash, three to four grains to the ounce of water, is one of the best applications which can be applied to the eye, in cases of purulent ophthalmia.

Tannate of Clycerine.-An excellent application to the eyes, in purulent opthalmia, and to moist sores, wherever situated upon the body.

Tannin.-This is the acid of barks, and is valuable, mixed with water, in running ulcers and sores, and in diarrhœa, in all animals. The powder sprinkled upon bleeding sores, will in many cases stop it. One drachm to a pint of cold water makes a good eye wash, and in many cases dries up the scum, and removes it altogether. It is certainly a cheap and useful remedy, and can do no harm to the eye.

Doses.-For horses and cattle, in diarrhœa, the dose of tannic acid is from half to one drachm, given in gruel as a drench. For calves with the same disease, give from ten to twenty grains às a dose, mixed with wheat flour gruel. Snuffed up the nostrils by man, in bleeding from the nose, it will in most cases arrest it at once.

Tannin of Krameria. - This is used for the same purpose as the preceding, but it is a better stypic, or arrester of bleeding from the skin or sores. 
Tar.-Pix Liquida.-Tar is not now used internally to the extent it formerly was. To cattle it is still given in a disease of the throat called clyers. For this purpose egg shells are filled with good Barbadoes tar, and suspended on the end of a split stick, and gently pushed down the throat of the ox. As an external application, tar is still used on sores about cattle, to keep the flies off, and is used to make stopping for horses feet singly, and mixed with clay, etc. It is kept in every horseshoeing shop for putting on the soles, and kept there with cotton, and a leather sole nailed on with the shoe.

OIL of TAR. - This is an excellent application when mixed with equal parts of sweet, or some fish oil, to promote the growth of horn of the foot of the horse. To give it a dark color to suit the foot, lamp black may be stirred in, in sufficient quantity to make a black hoof ointment, which will be found an excellent formula for this purpose.

\section{Tartar Emetic.-(See Antimony.)}

Tobacco.-Nicotiana Tabacum.-Tobacco is used as $\mathfrak{x}$ medicine, principally in skin diseases, and for the destruction of lice and other insects, in the wool of sheep. Tobacco smoke is a favorite remedy with some veterinarians, for the removal and killing of worms, and in constipation, and in colic. For these purposes, better and safer agents are in every day use. Tobacco in all, or any of its forms, is dangerous, being followed by great sickness, nausea and prostration, from which many animals are ultimately destroyed.

\section{Tragacanth.-(See Gum.)}

Turpentine.-Teribenthce.-The various varieties of turpentine in market. 
(1.) Common Turpentine-Is the produce of the pinus palustris of North Carolina, and other states, and also of Norway, and the north of Europe. The Pinus sylvestris, or Scotch fir, yields considerable turpentine.

(2.) Venice Turpentine-Is procured from the Larix Europea.

(3.) Canada Balsam.-This is procured from Abies Balsamce, and is considered to be the purest of all the turpentines; hence it is called sometimes, the balsam of Gilead.

(4.) Frankincense. - This is the product from Norway, and the north of Europe. This variety enters into the composition of what is known as Burgundy Pitch, and is used in veterinary practice for making adhesive plasters for the loins of horses, having been sprained, or having lumbago.

(5.) Oil of Turpentine.-This is commonly called the spirits of turpentine, and is used very largely in horse and cattle diseases, both for internal administration and external application.

Uses.--Oil of turpentine is a powerful stimulant, diuretic, and antispasmodic, and is therefore used in colic in the horse, and in puerperal apoplexy in cows, and in general debility in all animals, and as an external embrocation, it is very efficient when combined with an equal portion of sweet oil, but very dangerous ,when applied to the skin of the horse alone. The horse will become restless, and, in some cases, utterly unmanageable.

Doses.-The doses of oil of turpentine for horses and cows will be from one to two ounces always mixed with its own bulk of oil, especially for horses. For dogs affected with tape ${ }_{\text {w }}$ worm, half to one drachm is the dose, mixed with the yolk of an egg. 
(6.) Rosin-Or resin, is the residue left from the distilation of turpentine, and is chiefly used by horsemen to cause the horse to urinate more freely. Much mischief is often done to horses, by the administration of rosin. Indeed, most diseases of the kidneys and of the loins, can be traced to over-dosing with this substance. The more and oftener that rosin is given to the horse, the more the horse appears in the eyes of the driver to stand in need of his favorite dose. The kidneys become over-worked, the desire to urinate is more frequent, and less urine is roided each time, and the poor man does not see the mischief he has already done, but hastens to give the horse more to remedy the urinary defect he has thus been instrumental in producing. It will be well to remember that powerful diuretics, like powerful purgatives, tend only to weaken and debilitate. If there be a urinary defect, find out the cause, and if possible have it removed, and the effect will cease "causa sublata, tollitur, effectus," but do not attempt to do so by force, and against all science and common sense. Other preparations of turpentine have already been noticed. (See Tar.)

Urse Ursi-The leaves of the whortleberry, -was once in repute in diseases of the horse, but it has now fallen, and others have taken its place. It was used chiefly in diseases of the bladder and kidney.

Valerian.-Valeriana Officinalis.-IIowever useful valerian may be in nervous irritation in man, it is not so useful in veterinary patients. Camphor, and the gum resins, or assafœetida, answer our purpose better than valerian. 
Valerinate of Soda.-This has been found useful in chorea, or twitching of the muscles in the dog, in doses of from one to two grains. dog.

(1.) Valerinate of Iron.-A good tonic for the

(2.) Valerinate of Quinine.-An excellent remedy for agues in man.

Veratrum.-White Hellebore.-If we had not so useful a sedative medicine as we have in aconite, I would certainly recommend the tincture of white helleboreroot, in all diseases for which aconite is recommended, on account of its being more dangerous than aconite from an over-dose, I will not say much more about it, as wherever white hellebore is found in the country stores, aconite will be there also. Hence, there can be no good reason for recommending it when aconite can be procured.

Veratria.-The alkaloid of white hellebore, and is one of the most destructive of the deadly poisons. Nothing can be more so than this preparation of hellebore; and what is more, at present, no test is known whereby to detect its presence within the stomach or system.

Wax.-This substance is used for giving hardness and consistency to ointments, and is a favorite substance with me in the form of shoemaker's wax, for the purpose of filling up cracks and holes in the feet of horses, to keep out sand and dirt. To be applied as follows:Make the wax in the form of a stick, so that it can be held by one end, while you hold a hot iron to the other; and hold it over the hole or crack, so that the warm wax will drop into it. When the part is full, wet the finger, 
and by this means the wax can be smoothed, so that ordinary observers can scarcely observe a deficiency in the foot. The hole or crack must be perfectly dry, and free from moisture, or else the wax will not stick very long. When dry, and the wax put properly in, it will last for a month, or till the next shoeing time.

Willow Bark.-A much neglected, valuable and cheap medicine. This substance has within it a crystalline substance called salicine, which is an excellent substitute for the expensive quinine. Farmers and others will do well to gather this'bark in sufficient quantity, and have it dried; and in the spring of the year, or when any of the horses are weak, or out of sorts, take of the willow bark one pound, and boil in four quarts of water, till two quarts are left; then strain for use, and give a tumblerful mixed in cut feed, once or twice in the day. This will be found much better than the black snake root already spoken of under its proper head.

Yeast.-Brewer's yeast is one of the best applications which can be applied to an unhealthy sore or ulcer, as it immediately changes its look and smell. A mixture of yeast and charcoal is a domestic remedy not to be overlooked in the treatment of sores, especially in the summer time. The yeast and the charcoal are to be mixed in the form of a poultice, and used as such, occasionally changing it, so that full benefit may be given to the sore by its use. After the sore is cleansed and corrected, it may be then healed as a simple, healthy sore, by other remedies amply described through the book.

Zinc.-There are several preparations of zinc, which are used in the treatment of horse and cattle diseases, but chiefly in the form of external applications. 
(1.) Oxide of Zinc.-This is a white powder, like wheat flour, and is useful applied as a powder in greasy heels and other sores, which discharge a sanious fluid from them. Oxide of zinc is often used in the form of an ointment mixed with lard, and an excellent ointment is made by soap shaved down, and moistened with a little water, and oxide of zinc added to it. This is a good form to apply to greasy heels, and scratches on horses, which have to work every day: it keeps down irritation and fever, and, consequently, prevents swelling of the legs, and it is easily washed off, on the horse coming into the stable for the night.

(2.) Carbonate of Zinc.-This is used under the old name of calamine, either in the form of a powder or an ointment. The ointment is made by adding four parts of lard to one of calamine. Good for healing sores.

(3.) Sulphate of Zinc.-This preparation of zinc has already been described under the head of sulphate of zinc, (which see.)

(4.) Chloride of Zinc.-Butter of Zinc.-This, as well as the other forms of zinc, is not used as an internal medicine, except the sulphate, which is occasionally given to the dog as an emetic.

Chloride of zinc is a powerful disinfectant and deodorizer, and is used in the healing of wounds and sores. The solution is the usual form of using it. Two grains of the chloride of zinc to the ounce of rain water, makes a good eye wash. For ordinary sores, four to five grains of the zinc to one ounce of rain water, is a good application. One characteristic of the preparations of zinc when applied to sores is, no proud flesh can or will arise during their use. The chloride of zinc is used by some veterinarians as a caustic, but for this purpose better 
caustics, and cheaper caustic, will be found elsewhere described.

(5.) Acetate of Zinc.-This is made by aissolving oxide or carbonate of zinc in acetic acid. The chief use of the acetate of zinc is for the healing of wounds. In European stables it is used for the purpose of wetting bandages applied to old hunters and other horses, whose legs cannot be otherwise kept fine and free from swelling. The bandages usually employed in such cases are made of wash leather, which is so extensively used for drying carriages after being washed, and is called chamois leather.

Zingiberis.-(See Ginger.)

Zyloidine.-This is a white substance, which is insoluble, and is obtained from starch, and concentrated nitric acid and cold water.

\section{PRESCRIPTIONS AND PREPARATIONS.}

Cerates.-Used for dressing sores and ulcers.

(1.) Simple Cerate.-Take yellow or white wax, three ounces; olive oil, two ounces; melt them on a slow fire.

2.) Turner's Cerate.--Take of simple cerate five ounces; prepared calamine, one ounce; mix, and stir till cold. 
(3.) Cerate of Zinc.-Take simple cerate, six ounces; melt, and add oxide of zinc, one ounce, and stir till cold.

(4.) Resin Cerate.-Yellow Basilicon.-Take yellow rosin and yellow wax, of each half a pound, then add eight ounces of olive oil or lard, melt together over a slow fire, and strain through flannel cloth while hot. This is an old, but excellent application for sores, scalds, burns, etc.

Clysters.-For colic of all kinds, take warm water of about blood heat, one stable bucket full, four ounces of common table salt, soap sufficient to make a good lather or froth; of this, three to four quarts may be injected per rectum, every half hour till the animal is well. For the expulsion of the fundament bots, injections of flax seed oil is an excellent and safe way of removing them. Tobacco smoke injections are sometimes used for their removal, as well as in cases of constipation. To give injections of tobacco smoke, an instrument having both force and suction is necessary. Tobacco smoke is not recommended for either of these affections by the author.

Drenches. - Used in cases of colic. Take sulphuric ether, half an ounce; laudanum, two ounces; flaxseed oil, one pint: mix. Or take spirits of turpentine, one ounce; tincture of aconite root, twenty-five drops; aloes, one ounce in solution: mix. Or take warm ale, two pints ; ground ginger, half an eunce, tincture of aconite root, twenty drops: mix. Used in flatulent colic, accompanied with swelling of the belly. Take laudanum, two ounces; aloes in sulution, one ounce; chloride of lime, half an ounce: mix. Or take tincture of aconite root, twenty drops; aloes in solution, one ounce; sul- 
phite of soda, one ounce; mix. Or take spirits of hartshorn, three drachms; aloes in solution, one ounce; water, one pint: mix.

\section{Eye Lotions and Washes.}

Astringent Eye Lotion.-Take sulphate of zinc, five grains; rain water, one ounce: mix.

Lotion for Purulent Discharges from the Eye. -Take sulphate of copper, three grains; laudanum, ten drops; rain water, one ounces: mix.

For the Same.-Nitrate of silver, four grains; rain water, one ounce: mix, and apply with a camel's hair pencil once in the day.

Soothing Eye Wash.-Take extract of bellodanna half a drachm; rain water or distilled water, six ounces; dissolve and strain. This is to be used warm, as a fomentation to the eye.

Mild Astringent Eye Water.-Alum, four grains; rain water, one ounce: mix.

Sedative Eye Wash.-Take sulphate of atropine, three grains; rain or distilled water, one ounce; mix, and strain. The reader of this book will remember that belladonna, or atropine, when applied to the eye, will cause the pupil of the eye to dilate.

\section{Eye Salves.}

Nitrate of Silver Ointment.-Take nitrate of silver, twelve grains; dissolve in as many drops of water; simple cerate, one ounce: $\operatorname{mix}$.

Precipitate Ointment.-Take red precipitate of mercury, three grains; pure lard, two drayous: mix, and use when the eyelids are red and oozing. 


\section{Freezing Mixtures.}

Take sal ammoniac, five parts; nitre, five parts; cold water, sixteen parts: mix. Or, take sal ammoniac, and nitre, of each five parts; Glauber's salts, eight parts; cold water, sixteen parts: mix. The article to be cooled is to be placed in a tin vessel, which is to be placed in the mixture just as a butter kettle would be placed in ice water. By throwing a handful of sal ammoniac, or coarse salt or nitre, upon ice or snow, a low degree of cold will be produced, sufficient for all purposes. Freezing mixtures are of much benefit when applied to sprains, when ice cannot be had.

\section{Liniments or Embrocations.}

Liniments are external applications, and are used for the cure of sprains, bruises, chronic swellings, etc.

Liniment of Ammonia.-Take solution of ammonia, two ounces; sweet oil, six ounces : mix.

Liniment of Camphor.-Take camphor, one ounce ; olive or sweet oil, three ounces: mix.

Liniment of Aconite.-Take tincture of aconite root, two ounces; olive or sweet oil, four ounces; creasote, one ounce: mix. This is one of the most valuable embrocations which can be applied to a painful bruise, or sprain of whatever kind. It removes, and allays inflammation, irritation and pain.

Liniment of Lead.-Take soap (castile, five ounces; camphor, one drachm; Goulard's extract of lead, half an ounce; boiling water, one pint. Cut the soap into thin pieces, and the camphor in fine powder, and stir them into the Goulard's extract, then pour in the boiling water. 
Soap Liniment.-Opodeldoc.-Take castile, or even white soap, three ounces; oil of rosemary, two drachms; oil of thyme, one drachm ; camphor, one ounce; spirits of wine, one pint. The camphor is to be in fine powder, and the soap in fine shavings. The camphor, oils and soap are to be dissolved in the spirits of wine. Place the vessel, holding those articles by the fire till the soap is dissolved. It should be kept in wide-mouthed bottles, of from four to six ounces capacity.

Obszrve.-The oils of rosemary and thyme, entering into the mixture, is not so much for their medical virtues as for the fine smell. In horse and cattle practice, these oils can be dispensed with; or add the same quantity of olive oil.

Soap Liniment.-Common.-This may be used instead of the opodeldoc. Take soap in shavings, four ounces; camphor, in powder, one ounce; spirits of wine one pint; laudanum, half an ounce; and dissolve. This is a cheap and useful liniment for sprains and bruises.

Turpentine Liniment.-Take spirits, or oil of turpentine, two ounces; olive oil two ounces, mixed.

Lime Liniment.-Take equal parts of lime water and linseed oil, and shake them well together till a white, thick, creamy, or saponaceous compound is made. This is used in cases of burns and scalds, whether in man or beast. Spread some of the liniment on a soft cotton cloth, and lay ${ }^{\circ}$ the burned part, and when dry renew again.

Creasote Liniment.-Take creasote, one ounce, oil of turpentine, one ounce; olive oil, two ounces: mix. This is a favorite liniment for sprains, bruises, sores, and gives relief from pain when rubbed upon the sprained hock-joint and stiff joints generally. 


\section{Ointments.}

Blistering Ointment.-Take of Spanish fly in powder, one drachm; hog's lard, six drachms: mix. This is the ordinary blistering ointment. The old formula is one of the fly to four of lard. The weaker ointment answers the best purpose.

Red Iodide Ointment.-Take of bin iodide of mercury one drachm, hog's lard, one ounce mix. This is the most useful ointment the veterinary surgeon can use, or possess, for the cure of splints, ringbones, spavins, indurated or hardened tumors, and for the reduction of enlargements of the glands of the neck. Iodide ointment should only be used once in five days, or till the effects of the previous application have subsided a good deal. This precaution is to prevent a blemish being made by the hair falling off and not growing again, which should always be avoided.

Green Ointment.-Take of simple cerate or ointment, one ounce; and add one drachm of verdigris (diacetate of copper) and mix. This ointment will heal sores when other applications have failed.

Sulphur Ointment.-Take of iodide of sulphur, one drachm; hog's lard: one ounce, mix. Used in mange and skin diseases.

Zinc Ointment.-Take carbonate of zinc, one drachm; hog's lard, one ounce: mix. ' A good healing ointment.

Liver of Sulphur Ointment.-Take of liver of sulphur, one drachm; hog's lard, one ounce: mix. Used in mange and other skin diseases. A more cleanly and easily managed form, performing all its actions, will be one part of the liver of sulphur to eight parts of water. 
French Hoof Ointment.-Take of olive oil, wax, lard, honey and white turpentine, equal parts; dissolve, or digest over a slow fire. For black hoofs, a little ivory black stirred in while hot, will give it sufficient color. For blue feet, add sufficient Prussian blue or indigo, along with the ivory black till a blue tint is given to the ointment.

Common Hoof Ointment.-The hoof ointment sold for such, is composed of Venice turpentine wax, and suet in quantities sufficient to give consistence or thickness to the mass. Oil of tar and oil of olives, or some of the fish oils, equal parts, and sufficient lamp or ivory black stirred in to give thickness to the whole, is probably the best ointment which can be used to the hoof of the horse.

\section{Plasters.}

Plasters are not often called for in horse and cattle diseases, but some cases arise which call fur a plaster over the back or loins, such as chronic sprains, lumbago, etc. Take Burgundy pitch, four parts, wax one part, camphor, half drachm; Spanish fly, half a drachm; melt over a slow fire, and spread on soft leather and lay over the loins while warm. This plaster will be sufficient for all purposes where a plaster is indicated. Plasters should be worn six weeks, so that the horse will reap some benefit from them.

\section{Poultices.}

Carrot Poultice. - Take of washed carrots, boil till soft, strain off the water, and bruise the carrots into a fine pulp or mass; spread upon strong cloth. Used for sores of an unhealthy kind. 
Linseed Meal Poultice.-Take of boiling water one quart, and stir in sufficient linseed meal with a wooden stick or spoon, and beat it well so as to have no lumps. To be spread on strong cloth or canvas, and applied.

Yeast and Charcoal Poultice.-Take yeast, one pint; powdered charcoal sufficient to give consistence to the whole when well stirred in. This is a valuable poultice, and is not sufficiently known where it ought to be. Used in sores discharging a stinking matter from them, which it soon arrests.

\section{Tinctures.}

Tincture of Arnica. - Take arnica flowers, four ounces; alcohol, one quart; macerate, or steep for one week, and strain.

Tincture of Aconite Root.-Take of the dried and bruised root, four ounces; alcohol; half a pint; macerate for two weeks, and strain. A medicine no farmer can very well do without. Those who do without it, do not know its actual value. Twenty drops of the tincture of aconite root, under certain circumstances, is as valuable as the animal which may stand in need of its great curative virtues. Measured by its power in curing disease, its weight in gold is not its value.

Compound Tincture of Benzoin.-Commonly called Friars' Balsam. Take of gum benzoin, one and a half ounces; storax, one ounce; balsam of tolu, half an ounce; aloes in powder, two drachms; alcohol, one pint: macerate for one week, and strain or filter. Used for healing sores.

Tincture of Aloes and Myrrh.-Take aloes in powder, one ounce; saffron, half an ounce; tincture of myrrh, 


\section{2}

PRESCRIPTIONS AND PREPARATIONS.

half a pint: macerate for two weeks, and strain. Used for healing sores and wounds.

Tincture of Iodine.-Take of iodine, half an ounce; iodide of potassium, one ounce; alcohol, one pint: macerate for a day or so, and it is fit for use. Used as an injection for abscesses and empty cavities. It is also used by some for enlargements, in the same way as it is used for erysipelas in man, by painting the affected part.

\section{Spirits.}

Proof Spirits.-Take rectified spirits of wine, three parts by measure; water, two parts by measure; and mix. This is the proper strength for making tinctures. Keep the rectified spirits by itself till wanted, the water can be added when needed.

Spirits of Camphor.-Take of camphor, two and a half ounces; rectified spirits, one pint; dissolve. Used for sprains, bruises and sores.

\section{Waters.}

Lime Water.-Take a lump of lime, or limestone, of about a pound weight; lay it on a soup plate, and wet it with water, which will soon convert the hard stone into a fine powder. Then put it into a stone jar holding a gallon or upwards, and fill in one gallon of water, and cork it closely. It is now ready for use when wanted. The same lime may be used repeatedly, by pouring fresh water, as the other is taken out. This water is what the lime liniment is partly composed of.

Tar Water.-(Bishop Berkeley's Receipt.)-Pour a gallon of cold water on a quart of liquid tar: stir ; mix and work them together thoroughly, with a wooden ladle, or flat stick, for five or six minutes. Then let the vessel 
stand, closely covered, for three days and nights. Carefully skim the surface, without moving the vessel; pour off the clear solution, and keep it in bottles well corked for use. This is a useful medicine for man and horse in chest diseases, and it would be well worthy of a trial in chronic cough in the horse.

\section{MEDICINES TO BE KEPT ON HAND.}

The following medicines should be kept in every farmhouse, where there are horses and cows. If the farmer should not think it of sufficient importance, he should at

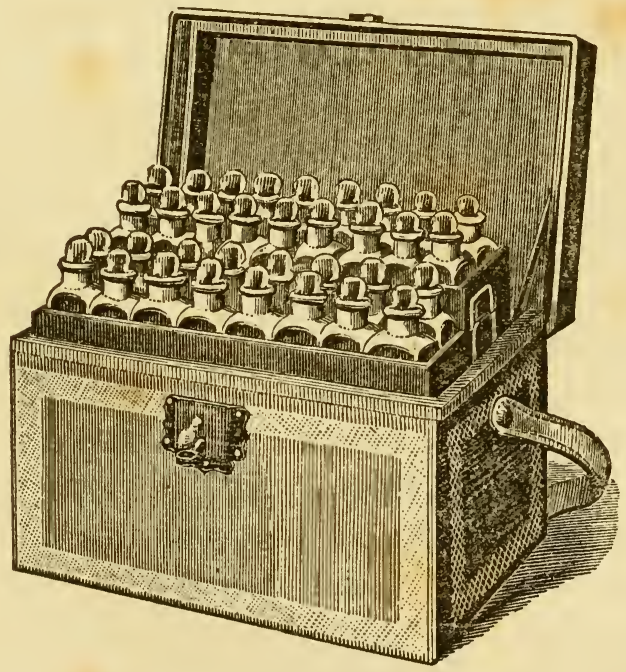

YEDICINE CHEST. 
least ascertain what is kept in the village, or other country store, so that he may know exactly where to get what he may at any moment stand in need of, for his horse or cow, in case of accident or sickness. Medicines should be kept in bottles, some in wide, and others in narrowmouthed ones. A plain label should be affixed to every bottle, and those which are known to be poisonous, a dark-colored label should be used, and marked Poison. This will often prevent mistakes and accident.

Tincture of Aconite Root.-One ounce.

Red Iodide of Mercury.-One drachm.

Tincture of Nux Vomica.-One ounce.

Spirits of Turpentine.-Two ounces.

Epsom Salts.-Two pounds.

Simple Cerate, or Ointment.-Two ounces.

Linseed Oil.-One quart.

Aloes.-One ounce.

Carbonate of Ammonia.-One pound.

Gentian Root.-Half a pound.

Sulphur.-Half a pound.

Olive Oil.-Half a pint.

Chalk.-Two pounds.

Powdered Opium.-One ounce.

Ginger.-One pound.

Fonugreek.-One pound.

Sulphate of Iron.-Half a pound.

Caustic Potash.-Six Sticks.

Glycerine.-Half a pound.

Lime Water.

Tannin.-One ounce. 
Catechu.-One ounce.

Sulphuric Ether.-Two ounces.

Blue Stone.-Two ounces.

Oxide of Zinc.-Four ounces.

Sulphuric Acid.-One pound.

Spirits of Salt.-One pound.

Sulphite of Soda.-One pound.

Spanish Fly.-Half an ounce.

Creasote.-Half an ounce.

Alcohol:-One pint.

Cotton, or Tow.-One pound.

Laudanum.-Two ounces.

Nitrate of Silver.-Two sticks.

Chloride of Lime.-A ftw pounds.

Powdered Charcoal.-A few pounds.

Black Oxide of Manganese.-Three ounces.

Roll Sulphur.-Half a pound.

Liver of Sulphur.-Three ounces.

[The above medicines can be obtained in their pure state from Mr. O. S. Hubbell, the celebrated chemist and druggist, 1410 Chestnut St., Philadelphia.]

\section{TABLE OF DOSES ACCORDING TO AGE.}

If the dose for a full grown animal, of whatever kind, be one ounce,
A colt of one year will require one-third of one ounce. 
A two year old will require one-half of the ounce.

A three year old will require three-fourths of the ounce.

The same proportion may be observed in cattle, from one year old and upwards. But a calf, a week or two old, will require another division of dose, but still following the same rule.

The dose for a one year old being one-third of the dose for an adult, or full grown ox. Then the dose for a calf of four weeks will be one-twelfth of that given to a one year old, and a two months' old calf or colt, will require one-sixth of that of a one year old animal. For a one week old animal, the dose will be one-fourth of that of the animal aged four weeks. The rule and the dose are merely approximate, but it is the best and only plan we can offer, as a rule or guide for a posological table. Some persons have advocated a decrease in the dose for old animals. This I cannot favor, as but few horses live to be so old that a full dose of medicine would be an injury to them. The great trouble with aged horses, is not from a failure of the constitution and the general system, but from defect in the teeth, whereby, in winter, old horses are nearly starved, as they cannot masticate their feed. If their food could be prepared, as is done for the old man, his life and usefulness would be prolonged; then it would be time enough to speak of diminishing the dose for old horses. 


\section{ARTICLES NECESSARY FOR MEASUR- ING, WEIGHING, ETC.}

One pair of scales and weights capable of weighing from one grain to one ounce.

Two glass measures, one measuring from five drops to sixty- the other from one drachm to six ounces.

One Wedgewood mortar, with pestle to match.

One tile for making ointment upon.

Two spatulas, or round-edged knives, for mixing and dividing powders, and mixing ointments.

A few small bottles with corks, to hold from one to eight ounces.

A few labels for pasting on the bottles, with directions for the use of the medicine.

A few labels with the word poison.

A few sponges.

One ox's horn cut sloping at the wide end for drenching horses or cattle, with which is better than a bottle. A bottle made of block tin, in the shape of a champagne bottle, is a good article for drenching with.

\section{Equivalents.}

A tablespoonful is equal to half an ounce by measure.

A dessert spoonful is equal to two fluid drachms.

A teaspoonful is equal to one fluid drachm.

A wine-glassful is equal to one and a half fluid ounce.

The surest way, however, to get the true quantity is 
to have the article measured by proper graduated weights and measures. It will not, however, be necessary to weigh each powder separately. Thus, half a pound of ginger can be easily divided into ten or twelve equal parts with a knife, without the trouble of weighing each powder separately. 


\section{PART III.}

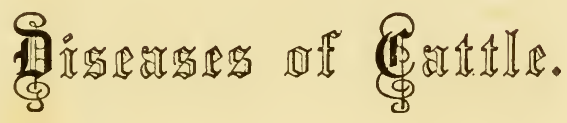



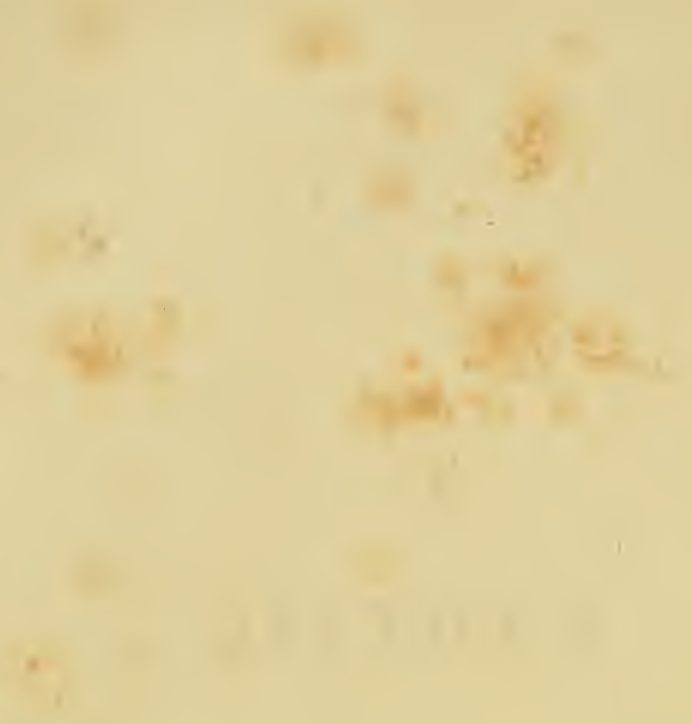

- 


\title{
DISEASES OF CATTLE.
}

\author{
PART THIRD.
}

This part of our work is devoted to the explanation and treatment of the various diseases affecting the ox, together with an account of the diseases incident to milch cows and to young calves.

Cattle diseases are, in many particulars, similar to those of the horse. Thus inflammation, irritation and fever, are common to all animals. Still, however, there are many diseases affecting cattle, in which we fail to find a counterpart in any disease attacking horses. Among such diseases we may mention those of contagious typhus, or rinderpest, epizootic aptha, or the murrain, spleenic apoplexy and quarter evil, or the black leg.

The manner or mode of treating disease in the ox differs no less so from those in the horse, than one disease differs from another. For it must be remembered by every person who undertakes to give medicine to an $\mathrm{ox}$ or a cow, that they have four stomachs-1, the rumen, or paunch; 2 , the reticulum; 3 , the manyplus; 4 , the abomasum. For this reason, or as it were peculiarity, cows or oxen should under every condition be treated with medicine in a fluid form only, so that it may pass 
from one stomach to another without injury, and in the shortest possible time. By giving medicine in the form of a solid, as is sometimes given to the horse, in place of the medicine finding its way into any of the stomachs, it is more likely to break through the floor of the oesophagean canal, (a platform at the bottom of the gullet) not only losing the medicine, but at the same time destroying the animal.

In cases of abscess, tumors, sores or ulcers, etc., on cattle, and where the description and treatment is not full enough, or not given at all, the reader is requested to turn to the Diseases of the Horse, or PART I of the book, where he will find ample information concerning what he may want to be more particularly posted upon.

Abortion.-This is the coming away of the calf, at so early a period, and before it is perfectly formed and matured, that it cannot live, and occurs at any time from conception, to within a few weeks of the full time of calving.

Causes.-The frequency of abortion, the apparently epizootic form which it sometimes assumes, together with the immense loss which thereby occurs to the farmer, and through him to the country at large, makes the cause of abortion in cows one of the most vital of subjects. The theory of sympathy, which some authors and farmers favor as a cause of abortion, is not to be entertained for a moment by any one having any pretension whatever to a knowledge of physiology, and the pathological anatomy of cattle. I have known a whole herd of cows, with but one or two exceptions, abort their calf, and yet not one of those cows ever saw one another. Some of those cows came from the State of New York, and yet they were kept separate by themselves, and from those 
already upon the farm. The owner had for this cause, and for the second time, sold out every cow on his farm. The first herd of cows, however, were allowed to associate with one another. The second herd was, as has been already stated, kept separate, but fed upon the same grass and hay, the produce of the same farm. So much then is this against the thenry of sympathy, not to speak of the utter impossibility for a cow to possess a power wisely withheld and denied to the higher and lower orders of the animal kingdom. For if this were not the case, how long could we be assured of the perpetuation and continuance of our own race, and of other species in the animal world?

Having thus denied the possibility of sympathy as a cause of abortion, let us now inquire what are the more immedixte causes, and after which we will endeavor, as best we can, to elucidate the more remote causes. The immediate causes of abortion are the separation of the appendages and attachments of the calf from the womb, and the contraction of the womb itself, whereby the calf is expelled to the outer world. The remote causes of abortion are few, and we will state them in the order of their importance. First, and by far the most important, and the one which is most likely to produce abortion in an epizootic form, is weakness and debility of the cow. This debility is the result of irritation of the fibrous, serous tissue of the body, occurring at seasons of the year, which generally produce, and give rise to coughs, colds and throat diseases. Hence, the epizootic form of abortion, so often seen, and attributed to sympathetic causes. This irritation and debility is of frequent occurrence, and is so insidious in its character, that even those persons most about the animals are not aware of anything being 
amiss with them. This is not strange, for we have heard, read and have seen animals in the worst forms of pleuro-pneumonia, and nobody thought of, or had seen anything wrong, worthy of observation or remark. It is but the other day, one of the papers of Philadelphia announced that a certain farmer had lost fifteen hundred dollars in a week or two, by the death of some cows which were not thought to be sick. It is only the true medical man who can estimate aright, the consequences of even slight irritation of the white membranes of the body. In this connection, it may be well to state, that cows in calf are more susceptible of debility than cows which are not in calf, for the simple reason that a great quantity of blood is required for the growth and maintainance of the calf in the womb.

This fact of itself, however, anomalous it may appear, goes very far indeed, in destroying that which nature so strongly endeavors to make perfect, or in other words, nature in this case defeats her own ends. Debility from the cow being in calf, and from the irritation of the shining membranes of the body, more especially of the windpipe, are the true causes of epizootic abortion in cows. The solids of the body become relaxed, soft and flabby, the placenta, or calf attachments separate from the fundus of the uterus, or womb, and the death of the calf from starvation, it is then a foreign body, the womb contracts and expells it, and this is called abortion. Farmers and others should not deceive themselves by thinking that when a cow is fat she is not weak nor out of order. Cows of this description are most likely to be the victims of irritation of the throat, and its train of consequences. Having thus defined what I believe to be the true cause of epizootic abortion, and which will, I think, upon fuller investiga- 
tion of the subject be fully borne out, I will now speak of the causes of sporadic abortion, or abortion occurring in isolated or single cases. A cow may, or may not abort after having suffered from an accident or injury, which will depend whether the calf or womb has been injured. A kick from a horse, or a gore from another cow is often the cause of abortion. The eating of diseased grass seed is another cause of abortion, but not so much, nor so often the cause as many persons suppose. For a cow will readily and without injury, take one ounce of the best and strongest ergot of rye to be found in the market; now where is the cow to get one ounce of ergotized grass seed or any other diseased seed?

There may be, however, other cryptogamic causes which we are as yet not acquainted with, and if we were aware of the existence of such, we are still ignorant of their action, if any upon the uterus of the cow; so I think we may as well dismiss this as being utterly untenable also. There is still, however, one other cause to be considered before we close this subject, and that. is, a cow having once aborted is ever after liable to do so again, and at about the same period of the previous abortion. The causes of this are local and confined to the womb itself, such as distention, dropsy, physometra, tubercle, fibrous tumor, polypi and hypertrophy of the womb; one or other of those diseases singly, or combined, are often seen in the womb of cows subject to periodical abortion.

Prevention of Epizootic Abortion.-When one or more cows have slunk, or aborted their calves, the other cows without a day's delay should have the following powder mixed in their feed night and morning for a week, and commence again and give every alternate week for a month or so. Powdered sulphate of iron, two drachms; 
powdered gentian root, half an ounce; mix and make one dose, to be given as above directed. This will be a powerful tonic, adding richness to the blood, strengthing the mucous surfaces, and imparting health and strength to the whole system, thereby enabling the animal to carry her young to its full time. Good and generous feeding is also a preventative by itself, but better in conjunction with the above powder. Cows having aborted from weakness and general debility of the system, can be prevented from aborting again by maintaining a high standard of general good health.

Abomasum.-The fourth or true stomach of the ox and of the sheep also.

Angle Berries.-Warty like excresences, and differ from the ordinary wart by having a stem or neck to them.

Treatment.-Cut them off with a knife or pair of scissors.

Anemia.-This term is applied to a week condition of cows sometimes called want of blood, and is more common than most persons are aware of. Cows are not selfish, the more a cow gets in feed, the more she will generally give in the form of milk and butter.

Symptoms.-White and clearness of the lining membranes of the nose, mouth and eyes are sure indicatior $\mathrm{s}$ of a want of blood in the system. When this condition occurs as an effect of disease as from the effects of pleuropneumonia, the animal will become hectic and die in a month or so from the first attack.

Treatment.-Give the following powders night and morning in cut feed. Powdered gentian root, three drachms, powdered ginger root, three drachms ; mix and make one dose. If those powders improve the animal in a week or so, give two drachms of the sulphate 
of iron in addition to the above. The iron, however, may dry up the milk considerably. The case may resolve itself whether it would be better to have a dead cow or a dry one. Good and generous feeding is indicated in this disease.

Apoplexy.-(See Splenic Apoplexy.)

Aptha or Thrush.-This is an eruption in the mouth similar to small bladders, and is often mistaken for a contagious disease called Epizootic Aptha.

Cause, Irritation in the mouth of young cattle from teething.

Treatment. - If treatment be at all necessary, a weak mixture of vinegar and cold water will answer, or a solution of alum or alum water applied to the mouth twice a day will be all that is wanted.

Biliary Calculi or Gallstones.-These are of several colors.

(1.) White Gallstones.

(2.) Yellow Brown Gallsones.

(3.) Dark Green Gallstones.-All of which are hard, grooved, and have the odor of musk. During the life of the animal, no symptoms are observed, whereby, their presence can be detected. This however, is of little, or no consequence as no treatment is necessary, and scarcely ever cause the ox any inconvenience.

Black Quarter.-Joint Felon Quarter Evil.-This is the disease of young cattle, and occuring in the spring of the year, and is characterised by one hind leg and thigh being congested, and black with coagulated blood. The other portions of the body retain their normal or healthy appearance. It is sudden in its attack, and death follow in a few hours. 
Causes.-A robust stamina or plethoric condition and fullness of blood.

Treatment. -No time for cure.

Prevention.-This is the most important particular to be attended to. On the first appearance of this disease, give each young animal less than two years, and those above one year of age, a good brisk purge to clean out the bowels. For this purpose, give half a pound of epsom salts in two bottles of water, sweetened with molasses, and add a teaspoonful of ginger. Put the young stock on high land, and where the grass is not too good.

Black Tongue.-(See Glossanthrax.)

Black Water.-(See Red Water.)

Bladder Diseases.-The ox is not free from disease of this organ. Bladder diseases in cattle generally, is the result of irritant matters in the urine, and from culculi or stone in the bladder.

Symptoms. - The animal is restless, pain and irritation constant endeavors to make urine, but only a few drops at a time allowed to pass. The eyes are blood-shot, and should the animal not be relieved, it will die from weakness and exhaustion from constant excitement and irritation.

Treatment.-Give at once twenty-five drops of the . tincture of aconite root to relieve irritation and pain. Give freely linseed tea and other demulcents, to soothe the kidneys and bladder. In orde: that any earthy matter which may be present in the uretus, kidneys or bladder may be lessened or destroyed altogether, give sixty drops of muriatic acid once or twice a day. This however, should not be given whilst there are pain or irritation present. 
Prolapsus of the Bladder-Cows, during and after difficult calving there will be sometimes observed a white, shining, watery bag protuding between the lips of the vulva. This is the bladder holding the urine, and is twisted at its neck, and therefore, cannot be emptied. Many a good cow has been destroyed by ignorant persons mistaking this for some of the watery membranes surrounding the calf, and have intentionally torn it asunder.

Treatment.-Carefully examine the distended bag, its shape and size, and having determined that it is the urinary bladder carefully cleanse it if dirty, and pass the hand previously oiled along its surface and acertain which way the turn or twist is male, having satisfactorily known this, take the bladder by the other hand and reverse the turn or twist, and then carefully push the bladder back into its place.

Should the bladder again return a tampon-a roll of soft cotton cloth should be used to plug up the vulva for an hour or two, and if this should not be sufficient, a stick or suture of stout saddler's silk, or other strong material should be passed through each side of the vulva with a small packing or darning needle and letting it remain for a few days.

Diseases of the urinary bladder are many, and diverse from one another, and having pointed out the most common forms of disease of this organ, I will simply mention by name others not so often seen, or at all events observed. Distension, causing abdominal pain, eversion, extroversion, inversion, causing painful urination, inflammation, paralysis, causing difficult, and in some cases entire stoppage of the urinary flow and rupture of the bladder, polypi in the bladder, causing frequent urination, and the urine small in quantity. Milch cows are chiefly the subjects of 
those last varieties of diseases of the bladder, and is the cause of many mysterious deaths occuring amongst them.

\section{Blood Shot.-(See Quarter Evil.)}

Blood Diseases.-(See Quarter Evil, Red Water, etc.)

Brain Diseases-.Phrenitis.-Or inflammation is the same as mad staggers in the horșe, and is due to overfeeding and distention of the stomach, by long wet grass or clover, and in rare instances it is caused by the deposit of wax-like matter within the ear, and communicating with the brain.

Symptoms. - First, the animal is dull during congestion ; this is succeeded by quickened breathing, excitement and delirium, the eyes are blood shot.

The animal is the picture of disease and suffering, and if not soon relieved, falls and remains in a state of stupor, from which it never comes out of.

Treatment.-In the early stages of this disease, much can be done by an active cathartic or purge, composed of one pound of epsom salts, and one pound of table salt, dissolved in four quarts of cold water, and sweetened with molasses. Apply ice or ice water to the fore head, and warm water bandages to the legs, and tightly clothe the body. Inflammation of the brain is often seen in the fatal form of milk fever in cows.

Brittany Cow.-A very small animal, and is imported from the south of France, and from Brittany itself, which is to the north west of France, and separates the English Channel from the Atlantic Ocean. M. P. Bellamy, Veterinary Professor to the Department of Agriculture, at Renncs, says La Vache Bretonne Utile au Riche, Providence du. 
Pauvre-Or the brittany cow, useful to the sick, and a blessing to the poor. Some few of those useful little cows, have been imported into this country, and if all be true which is said in their favor, more of them ought to be imported. They are represented to be hardy, healthy, and can be kept on such food as another cow would starve upon, and they can stand a greater amount of hardship, than any other variety of cows.

Bronchitis.-This is inflammation of the wind-pipe, and even extending to the lungs themselves. There are every variety or severity seen in this disease, from the simple irritation of the buccal membrane to the intense inflammation terminating in the outpouring of serum from the blood into the cavity of the chest, resulting if the cow be in calf, her abortion, and in others the death of the animal, after a long and lingering hectic fever. Bronchitis, throat and chest diseases in cattle, are insidions and deceptive, for the ox does not, even under the most severe forms of throat and chest diseases, exhibit fever and irritation, loss of appetite, etc., which are shown at once in these diseases, when attacking the horse. Not until the disease has made considerable progress does the ox or the cow show symptoms of disease; so much is this the case, that I have on several occasions pointed out bronchial and chest disease, in milch cows, whilst the owners failed to see anything wrong. Indeed, this is the great difficulty in the successful treatment of diseases in neat cattle-not getting, or applying the remedies in early season of the affection. A greater and more strict attention, and observation on the part of farmers and others, is almost imperatively demanded, and more especially should this be the case with dairy people; for when anything is at once dis- 
covered, such as a very slight grating sound, being heard in the wind-pipe, when the ear is steadily applied. 'The cow should immediately have some iron, or tonic powders, and all will be well in a few days again; whereas, if left to itself serum is exuded, and debility, weakness and death itself, may follow. I would say to dairy men, study the sounds in the wind pipe, both in the sick and well animal, and you will not only perfect your knowledge in this particular, but I assure you it will be no idle study, for in a short time you will not only be able to detect those insidious diseases, in the early season, in your cows, but can apply the remedy also, thereby saving yourself from inconvenience and loss, which some of you can ill afford, and at the same time you can prove to your neighbors, and to the world at large, that cattle diseases are not so difficult to cure after all, and that we have so long sat and brooded with soured and sullen minds over our losses, and the ignorance and inefficiency of cow doctors, without avail.

Cause.-Bronchitis generally is a disease which rarely attacks one animal only, but the most of the herd will have been attacked before it leaves the place, and then it will leave when there are no more victoms for it to seize. Those epizootic diseases depend upon what is called atmospheric causes. This condition usually manifesting itself in the spring of the year, and sometimes early in the summer. This peculiarity of the air causes irritation of the fauces of the mouth, throat or wind-pipe, and as before stated, sometimes extends to the chest and lungs themselves.

Symptoms.-In a week or so after the attack, a slight liusky cough, weeping from the eyes, and a watery discharge from the inner corner of the nose will be seen, and by applying the ear to the course of the wind-pipe, a 
slight, rough and grating sound will be heard. This sound, however, can be heard from twelve hours after the attack. According to the amount of serum poured out, and whether the cow be in calf, and how far she is gone in calf, so will the quickness and depth of the breathing be. Bronchitis is the forerunner of pleuro-pneumonia, commonly called the "Massachusetts Cattle Disease." The spotted and solid appearance of the lungs of animals having died of this catttle disease, is due to the lungs being so long immersed in the serum or fluid, which is poured into the chest. Pathological anatomists are well aware, that if the substance of the lungs had been the original seat of the disease, the animal so affected would either have died, or have recovered in about forty-eight hours; whereas none scarcely die within the first week from the attack, and many live a lingering life of six weeks and two months. This fact cannot be reconciled with the current theory of the lungs being diseased by direct attack. No! They are solidified from their functions being impaired by being immersed and surrounded by fluid poured out from the fibrous serous tissue, or from the effects of inflammation of the linings of the windpipe, and perhaps the linings of the chest, singly or conjointly with that of the windpipe also. This I am satisfied is susceptible of no other theory, or explanation whatever; and the sooner that farmers and others think so too, it will be the better for them.

Treatment.-If the disease be discovered within fortyeight hours from the attack, give four to five doses of the tincture of aconite root, twenty-five drops to a dose, and give one dose every four hours. If there be no certainty that the disease has existed longer or shorter, and to save time, the aconite may be given along with 
the following powders three times in the day. Powdered sulphate of iron, three drachms; powdered gentian root, half an ounce; powdered ginger root, half an ounce; powdered sulphite of soda, half an ounee; mix and make a drench to be poured down the mouth out of a strong bottle. This medieine is to be eontinued (omitting the aconite after the fifth dose) till the animal is well, or looks more bright and ants all it gets: and if it be a milch eow, the usual quantity of milk will be given. In addition to the above medicines, give once or twice daily, half an ounce of eommercial sulphuric acid, largely diluted or mixed in half a bucket of cold water. In feeding the animal, eare should be taken not to give too much, so as to bring on dangerous indigestion. Cold water and pure nir are indispensable agents in the treatment of this and all diseases of horses and eattle.

Bull Burnt. - This is a disease similar to gronorrhoa in man. (Of all the domestie animals, the ox tribe are the only ones which are subject to this disoase, more common in hot than in cold weather.

Symptoms.-In urinating there is uneasiness, and the urine is passed in small quantities, slight mucous discharges from the organ of generation. In the bull, there are at times no desire for eopulation, even when it is desirable that there should be. This often leads to the diseovery of the cause.

Treatment.- Separate the bull from the cows, and give the affecterl ones, including the bull, a purgative of Epsom and common salts, one pound each, mixed or dissolved in four quarts of cold water swectened with molasses, and add a little ginger, say a quarter of an ounee. Be assured that both bull and cows are thoroughly cured before 
the bull is admitted among the cows, for a second attack on the bull will render him useless.

Cachexia.-This, properly speaking, may be denominated a bad habit of the body, from faulty digestion, and assimulation of the food taken into the stomach.

Symptoms.-Morbid or diseased appetite, the animal at every chance will lick the earth, stones, lime walls, and other substances, which cows in health would not touch. This disease, like many others, occasionally takes an epizootic form, and attacks animals over a large tract of of country. More particularly, the animal's coat stares, followed by a mucous discharge from the eyes and mouth; milk almost disappears, and what there is of it is very blue and thin. At this stage of the disease, the animal is thin; the membranes of the mouth, nose and eyes are white and pale, showing the bloodless state to which the beast is reduced. There is now stiffness of the joints consequent upon infiltration of serum into the bursæe of the joint, causing much swelling. In certain parts of England it is called the "cripple." The animal is now reduced to a skeleton, and dies within a few months to a year from the first appearance of the disease.

Treatment.-The first thing to be done is to have the animals removed to high and dry pasture lands, which may be the means of cutting short the disease, before nuch injury has been done to them. Give to each animal plenty of salt, and the preparation of soda and lime, with good food of a nutricious quality. A few doses of commercial sulphuric acid highly diluted, say half an ounce by weight, given once a day in half a bucket of cold water, and if necessary, a few doses of iron and gentian, will cut short the disease. 
Caiving. - This is an operation of nature, which most dairymen and farmers are familiar with, and upon which we will say but little. Before we do so, however, a short description of the symptoms, by which it is known when a cow is in calf, will be given.

Symptoms.-The first and most important symptoms of a cow being with calf, is the absence of ostromania or bulling. This, however, is not altogether relied upon in some cows, for there are cases in which a pregnant cow will receive the bull up to the time of calving. The next, and probably the more sure sign of a cow being with calf, is the increased size of the belly. The hand placed firmly against the flank or portion, where there are no ribs; a hard, firm body will be felt, which is the calf. As time passes along, the movements of the calf in the womb can occasionally be seen. Within a few weeks of calving, the external organs of generationlabia pudendi-increase in size, and discharge a thick mucus. The udder becomes swollen, hot, and full of the first milk-colostrum, which is sure indication that the time for calving is near at hand. This is accompanied by a relaxation of the ligaments of the pelvis, or as the dairymen say, she is down in her bones.

Symptoms of Immediate Delivery.-Great restlessness and uneasiness, lying down and rising up, increasing in severity, until she has been delivered of a calf. From the time of conception, till natural parturition or calving is about forty weeks, or two hundred and eighty days.

There are, however, opinions at variance with these dates, founded upon the sex of the calf. If a male, it is carried longer than if of the opposite sex. These opinions form ground for debate, therefore we will drop theorizing. 
Natural Calving. - This resembles the fruit when ripe, breaking from its attachment to the parent tree, and falling into the lap of mother earth. If the pelvic bones are well or proportionably formed in the cow, and the presentation and size of the calf be right, no trouble need be apprehended, and the cow may be left to herself. The natural presentation at full time, and of a full grown calf, is as follows: The amnion, or waterbag having been ruptured or broken, there will be seen the fore legs and head of the calf resting upon the two feet, and protruding a little. If, however, the cow should labor long with the calf in this position, and she is weak and thin in flesh, she may be assisted: this can be done by taking hold of the two feet and part of the head, and when the cow makes an effort to strain, pull at the calf. If sufficient force cannot be applied by the hands, then place a soft but stout rope round the feet of the calf, and apply traction or force. Before any force whatever is used; be sure that the mouth of the womb is sufficiently dilated; for if this. be not the case, then by using force the womb is torn or ruptured, and the death of the cow is the result. Be patient, and time, the prover of all things, will also prove the wisdom of waiting. If after delay, however, both cow and calf should be lost, be consoled by the fact, that it is likely the one or both would have been lost any way; for there will be found some one or other of those malformations, either in the pelvis or arch of the pubis of the cow, or an undue proportion of the size of the calf to the pelvic arch through which it is has to pass, to be delivered. In all cases where the calf presents itself in the form above described, and delivery of the calf is not effected, rest assured that the pelvic arch of the mother 
is too small or too narrow. If that be not the case, the calf is over size, from water in the head, (Hydrocephalus,) or water in the belly, (Dropsy,) or both of these conditions combined, which is seen by veterinary surgeons, in large country practice.

Before a calf of this description can be delivered, and the cow relieved, the head of the calf must first be pierced, to lessen its size, then a long spear-like instrument-trocar, is used to pierce the belly or abdomen of the calf, to empty the fluid, to lessen also its great size. When that is properly done, and the cow is not too greatly exhausted, she may complete the delivery without further assistance.

Twin Calves.-Twin presentations are as various as they are curious. Thus, we sometimes see a fore leg of one calf, and the hind leg of the other. In this condition of things it must be evident that no force should be used to bring them away till each calf is properly adjusted in its position. The best and quickest way is to adjust the calf which first presents itself, and if portions of the other be presented also, push them back into the womb. One calf safely delivered, not much difficulty will be experienced with the other.

Unnatural Presentation.-We have now arrived at that portion of our subject, which more plainly talking means, when the calf is being forced into the neck of the uterus or womb in any form, whatever except the natural position already described. The unnatural position of calves, about to be delivered is happily rare, and forms the exception to the natural law of presentation. However rare these presentations are, they nevertheless are sometimes seen. Thus the feet are pre- 
sented, and the head of the calf doubled on its neck, and in the womb. This condition is best remedied by tieing a rope round the fore feet, or both together, and raising the hind parts of the cow off the ground; thus forcing the calf down to the fundus or bottom of the womb. This being done; reach the hand in and seize the calf by the head, and bring it with you, while an assistant is puiling upon the rope attached to the fore legs.

Byadopting this plan a safe and speedy delivery will be effected. English veterinary surgeons, with Professor Simmonds at their head, say this is the most dangerous form of presentation, involving the life of both cow and calf. Why do they say so, and how does it prove so to them? because they vainly endeavor by force to bring the head of the calf into the neck of the womb, when there was no room do so, without first elevating the hind portion of the cow sufficiently for the calf to fall to the bottom of the womb where there is plenty of room to turn, not only the head, but the whole of the body also. Another form of malformation is, where we have the head and neck within the neck of the uterus or womb, without the fore feet and legs. The only alternative in this case will be to remove the head by cutting it off from the neck; leaving, however, sufficient skin for attaching a rope to it. But if the head has not fairly passed out, an attempt should be made to put it back into the womb again, and not bring it out. Before pushing it back, fix a rope round the lower jaw. The legs one by one should be searched for by the hand, and when found secured by another rope. After both fore legs and head are thus fairly within your grasp, then use traction or gentle force, and at every strain made by the cow take advantage of it, and by these means the calf will be safely 
delivered. The next unnatural form consists in the presentation of one or other of the fore legs, where the nose is pressed downwards, and the crown of the head only is seen or felt. Secure the leg which is seen by a cord, push on the crown of the head or elevate the hind parts of the cow a little, to force the calf slightly down in the womb; then pass a cord round the lower jaw, and the other fore leg must also be found, and secured by a rope. The rope attached to the jaw should be first pulled to straighten the head on the neck, then the cord attached to the legs should be pulled simultaneously, and the head and feet as they are presented should be guided by the hands of an assistant until the head and both fore legs have fairly entered the proper channel.

The next presentation is what is called a breech presentation-the tail and buttocks are here presented for delivery, which, of course, cannot be effected in such position. The only chance of a safe delivery is to get hold of the hind legs, which is not easily effected; but perseverance, assisted by ingenuity, can do much, when it is well known what is required. In this case, it is to get hold of both hind legs. When the hind feet and legs have been secured, and brought into the proper channel, delivery can be easily effected.

The next and last presentation which we notice; is where the crown of the head is presented, and the calf lying upon its back. This is a tedious labour, and to effect a delivery at all, the calf must be turned, which by the by is not an easy task; still, however, it can be done by securing the head and fore legs with a rope. Should all efforts fail, do not exhaust the strength of the cow, but hasten to detach the legs of the calf, opening its belly, and in some cases, the head also, to 
lessen the size of the calf, thereby securing the delivery of a mutilated calf, but a living cow. The subject is far from being at this point exhausted, but we have gone sufficiently far for our pages: we will now proceed to another subject connected with calving, and that is the retention of the after-birth, or retentio secundinarium, as it is called, or, as the dairy folks say, the cow has not yet cleansed.

Retention of the Placenta, or After-birth.-If the cow has gone her full time with calf, and is in a healthy condition, the after-birth will not be retained long after she has given birth to her calf. When a cow does not cleanse properly, and within a reasonable time there is then something otherwise wrong with her health, such as debility and want of vitality in the system: it is this that must be looked to, and not the want of timely cleansing that demands attention, as being the cause of the cow not doing well after calving. Remedy these existing causes, and the cow will cleanse properly enough. Contrary to the generally received opinion of farmers and others, the retention of the after-birth for a day or two will do no harm, provided that decomposition does not take place with the after-birth; for in such cases, the whole system of the cow is apt to be contaminated and poisoned.

Treatment.-Cows not having cleansed properly within twenty-four hours after calving, should take the following mixture given:-Epsom salts, one pound; powdered ginger, one ounce; powdered fœenugreek, one ounce; carraway seeds, half an ounce; mix, and give in three or four bottles of warm ale, porter or warm water, sweetened with molasses. This mixture not having the desired effect in twelve hours, the hand, well greased, should be intro- 
duced, and gently press the after-birth at the attachments, called cotyledons: this must not be accompanied with much pulling, pressure with the finger and thumb will be all that is wanted. This operation may be followed by giving the cow a little warm ale or molasses water, with half an ounce of powdered ergot of rye, and in half an hour, half an ounce more may be given. This will cause contraction of the womb, and expulsion of the placenta. When decomposition or putrefaction of the afterbirth has taken place, which is known by the black color, the womb should be well washed out with a weak solution of chloride of lime, and administer by the mouth one ounce, three times in the day, sulphite of soda for a week, to prevent, if possible, and neutralize any of the poison of putrefaction, that may have been absorbed into the blood. Give the cow good and nutritious feed to support her strength.

Symptoms of Blood Poisoning.-The absorption of putrid matter into the blood is immediately followed by fever, of a low type, called typhoid, and if not speedily checked will be succeeded by typhus, from which the animal cannot recover.

Treatment.-Give the sulphite of soda in the manner spoken of above, combined with two drachms of the sulphate of iron, and one ounce each of powdered ginger and fonugreek three times in the day, till the beast is bright, lively, and free from stupidity.

Inversion of the bladder will be found treated of under Bladder Diseases.

Disease.-Inversion of, or falling down of the calf bed, will be treated of under its proper or alphabetical head,-Falling of тне Wомв. 
Catarrh.-This is not a disease common to the ox, for when causes arise sufficient to produce catarrh or cold in the cow, it does not run its course as such, but is speedily accompanied, or at least followed, by severe complications. Cattle not exceeding two years old are subject to a disease somewhat similar to cold, and is called hoose.(See Bronchitis and Hoose.)

Catarrhus Linuum Frontalium.-Malignant $\mathrm{Ca}$ tarrh.-This is one of the recorded diseases of the ox which I think has really no existence, except in the brain of some men, or it may be a condition confounded with Typhus Contagiosus Boum. The disease is described as being similar to glanders in the horse. If this be the case, the disease is not properly named; for the frontal sinuses are not the seat of the disease. This multiplication of names of diseases is only adapted to puzzle people, and annoy the farmer. If this be not the true reason, then I unhesitatingly denounce those writers as being utterly ignorant of the first principles of pathology, upon which all writings and opinions should be subservient.

Cattle Plague.-This is a vague name, and conveys no intimation of the cause, nature, seat, or characteristics of the affection, whatever it may be. Therefore, for the credit of the writer and for the convenience of the public, no disease should be called a plague. There is now no disease affecting the human family called a plague. The experienced physician can now tell the nature, seat and complete history of the disease, and gives it a name by which it will ever henceforth be known and recognised. Why should not the veterinary surgeon do likewise?

So long as such men as Gamgee continue to write 
and speak of cattle plagues, so long will veterinary surgeons continue to labor in vain for the public confidence. Why should not things, states and conditions be called by their right names? Cattle disease, cattle plague and rinderpest should long ago have been blotted out of the books; for at best, they only serve as a cloak or cover to hide the innate ignorance and stupidity of some veterinary surgeons, horse and cow doctors particularly. The word plague means a stroke, and that is all the insight a person can derive from the word. As every disease may be considered a stroke, why not add what kind of stroke it is, whether it be a stroke of palsy, or of the sun?-For Cattle Plague.-(See Typhus Contagiosus, Boum and Pleuro-pneumonia.)

Chicken Pox.-Varicella Boum.-A pustular eruption on the teats of cow's presumed to be caused by eating vine leaves. The eruptions soon discharge, dry up and heal, without any treatment whatever having been applied to them.

Choking.-This is of frequent occurrence among cattle or cows fed upon potatoes, turnips, etc.

Treatment.-When the potato is lodged in the upper or middle third of the gullet, the mouth of the animal is to be held open by means of a balling iron, or some other contrivance, while a person having a small hand should pass a cord like a clothes line, with a loop on the end of it, and try to get the noose over and beyond the obstruction. If the substance be low down in the gullet, manipulations may be tried from outside, by tightening the skin upon the obstruction, and trying to move it up, if possible; but downwards, if it will go without too great a force being used. If this fail to remove it either up or down, try 
to dislodge it by pouring small quantities of oil or melted lard, not hot, down the throat, and having failed in all, the probang should be used, or in its stead, a strong flexible cane or rattan, may be tried; but care should be taken to have the cane go down the right passage. If coughing is set up on the introduction of the cane, have it withdrawn as it has entered the air passages, but try till it has been properly entered down to the obstruction. With patience and perseverance the difficulty will usually be overcome. Still, however, there are cases which require the gullet to be opened over the place of obstruction, a safe operation requiring only a simple cut through the skin and outer surface of the gullet, which will readily heal without much trouble, by bringing the lips of the wound together, a stitch or two with strong, but small twine or saddler's silk, by means of a small packing needle. Feed the cow or ox, for a week or more, upon soft or prepared food till the wound has healed. Cows or oxen remaining long in a choked condition, the throat is apt to swell from the accumulation of gas in the first stomach, which will have to be treated as for hoven or tympanitis, occurring from eating clover or rank and wet grass.(See Hoven.)

Colic.-Colic in cattle is rarely seen as it is in the horse, but occurs in the form of hoven.-(Which see.)

Consumption.-This disease is not so common in domestic animals as in the human family, nor is it as frequent in any of the animals as it is in the milch cow. Consumption in cows is usually exhibited in the tubercular form. These tubercles are from the size of the pin head to that of a hickory nut, flattened, oval and round, and are not confined to the lungs, but they are 
seen underneath the pleura costalis lining the ribs,over the diaphragm, peritoneum, and the omentum or caul.

Causes.-This is considered one of the hereditary diseases of cattle, or rather milch cows. As is elsewhere stated,-in the first part of this book,-hereditary disease does not necessarily imply that a consumptive cow should be so, because its mother was consumptive. No. But because it has inherited the great milking qualities of its ancestors, whereby the animal is reduced in flesh, condition and vitality, the fibrous, serous tissue of the body becomes deteriorated, and hence the foundation is laid for tubercular consumption. Cows of some breeds are not selfish, for the more they are fed, the more milk will they give. These are all the claims which can justly be made in favor of the hereditary cause of consumption in cows.

Symptoms.-Thin of flesh, unthrifty, and staring coat or hair, long and dead-looking, a low husky cough, loss of appetite, weakness, and a bloodless condition of the whole system, which is readily known by the pale, white look of the lining of the eyes, nose, and mouth. The consumptive cow stands with her back arched, and her forelegs turned out at the elbows, and when lying rests on the belly and breast bone. The milk of such cows is thin, blue, and watery.

Previously to the death of a consumptive cow, diarrhœa, dysentary, discharges from the nose and eyes, accompanied with hectic, or sympathetic fever, conclude the symptoms of this lingering, but fatal disease.

Treatment.-In the early stage of consumption in cows, much can be done in the way of treatment. Feed the animal well with rich and nutritious material, such as 
linseed, cake meal, etc. Sulphuric acid given several times a week, in doses of half an ounce by weight, largely diluted with water, will sustain the vital powers, and impart tenacity to the buccal membrane throughout the body. Occasional doses of sulphate of iron and gentian; three drachms of iron and half an ounce of the gentian, will make one dose. Such treatment will prolong the life, and at the same time improve the condition and milking qualities of the cow.

\section{Boum.)}

Contagious Typhus.-(See Typhus Contagiosus

\section{Coryza.-(See Catarrhus, Linuum and Hoose.)}

Cow Pock.-It will be unnecessary for me to enter into the discussion of a subject, which is so familiar to the ears of so many, and which is of so much benefit to the human family as cow-pox.

Cow-pox is a specific pustular eruption on the skin of the teats and udder of the milch cow. It is not a dangerous disease, although at the same time, it is a contagious one, and easily communicated to man or milk maid. This discovery, together with the immunity which is insured from the natural or small-pox, has imparted the stamp of immortality upon the name of Jenner.

Croup.-Stridulous croup in animals is rare, but it is seen occasionally in milch cows, and is very fatal, from the fact that it is situated in the larynx, which speedily causes suffocation, unless the windpipe be opened with a knife, to admit of the act of respiration and expiration, till the swelling of the head of the windpipe has passed off.

Cause.-Cold attacking the head of the windpipe, fol- 
lowed by inflammation, and the development of false membranes.

Symptoms.-Loud, stridulous noise or murmur, quickened breathing, excitation, fever, and threatening suffocation of the animal, cough and distress.

Treatment.-Place the animal in the open air, if in summer time, in the shade, and give aconite in the form of tincture, twenty-five drops to a dose. This will allay the excitement, fever and irritation. If this give relief, repeat the dose in a few hours again. But on the contrary, there being relief in half an hour, give no more aconite, nor indeed any thing else. There will be but one of three things to be done: either to kill the beast, if it be in good condition, and fit for market; wait for the animal to die, or have the boldness to cut out a hole in the windpipe, about the middle, and on front of the neck. Tighten the skin on the front of the windpipe, and make a clean cut fair down the centre, and through the skin; when the white shining windpipe is brought to view, have an assistant to hold the edges of the skin back out of the way, till a hole is cut out of the cartilages of the tube, as large as a fifty cent piece. This will give instantaneous relief. The hole will gradually fill up, and close again without any trouble whatever. This, I am satisfied, is the only sure way of cure in this disease.

Cud, Loss of.-This occurrence is the symptom of, and not a disease. Loss of the cud, or rumination, accompanies almost every disease of any importance attacking the ox or cow. When rumination has ceased for a time, and is resumed again, it is a good symptom that the animal is somewhat better, and an indication that the functions of the body are about being resumed again, and are demanding food for their nourishment. Loss 
of cud is among the first symptoms observed by farmers in case of bronchitis, pleuro-pneumonia, hoven, aphtha, etc.

Diarrhœa Simple.-This variety does not call for much description, as it is only an effort of nature to get rid of something that is injurious. By its removal the purging will stop. Simple diarrhœa rarely calls for treatment, but if it should, change of feed and pasture land will be the first things to be attended to, and if necessary after a trial of new feed and pasture, a few powders composed of prepared chalk, two ounces; ginger, half an ounce; opium, one drachm: $\operatorname{mix}$ and give in the form of a drench, with wheat flour gruel. Repeat the dose, if it be necessary, but never be in a hurry to give astringents in looseness of the bowels, as much mischief may be done by controverting the efforts of nature, which are always of salutary effect, if not too violent for the condition of the beast, and in that case interference is now necessary.

(1.) Diarrhas Chronic.-This condition is sometimes called the Rot, from the belief that the animal is rosten. Chronic, or indeed, any kind of diarrhœa should not be looked upon as a disease at all, but merely a symptom of internal irritation of some kind.

Causes.-This is an important inquiry in cattle patho$\log y$, for farmers are sometimes disappointed when they are told the animal will die within a given time, not knowing the nature of the disease of which diarrhoa and dysentery are but the symptoms. Chronic diarrhœa is the sequel to tuberclulous consumption already described under that head. Or dysentery may show itself before even consumption is either thought of or recognized. However, chronic diarrhoa or dysentery is the result of tubercles 
situated on, and in the white membranes throughout the body.

Treatment.-Generous diet, composed of linseed or cake meal, commercial sulphuric acid, gentian, sulphate of iron, are the medicines indicated. In addition to this treatment, which has already been recommended for consumption, I would advise weekly inhalations of sulphurous acid gas in the manner recommended under that head in the list of medicines in the second part of the book (which see,) for I think this is one of those diseases which will be greatly benefited by its proper use.

(2.) Diarrhag in Calves. - This is a frequent affection among young calves, and destroys thousands of them every year.

Cause.-Depending upon the character of the milk; not so much in regard to its quality as it is owing to the time and manner of giving it: thus, calves are not allowed to suck their own mothers;-frequently they are not allowed to suck at all, but have to drink the milk out of a bucket, and then it is often cold before the calves are allowed to have it. The rapidity with which they drink their allowance, which is often too much for them, gorging the stomach and paralyzing the digestive functions, and hence, the white diarrhœa so often seen among young calves.

Symptoms.-The symptoms one would think alike in all animals; but this is not the case here, or so far as the diarrhœa of calves is concerned: voracious appetite, swelling of the belly with occasional pain, discharges of wind or gas, and white or yellowish colored excrement or dung, and in some bad cases the true milk is passed unchanged by the action of either stomach or bowels.

Prevention.-This is better than cure, and consists iu 
allowing the calf until several weeks old to suck its own mother, not only morning and night, but at least three times in the day, and divide the periods as evenly as possible. Thus by allowing calves to suck the milk for themselves paralyzation and gorging the stomach with cold milk is avoided, and thereby white diarrhoea is prevented.

Treatment.-Give three drachms of carbonate. of soda in well boiled wheat flour gruel once a day; and if this is not convenient, give a tablespoonful of common rennet after each feed of milk the calf takes; this will materially aid proper digestion by its power in decomposing the milk and fitting it for assimilation.

\section{Distention of the Rumen.-(See Hoven.)}

Dropping After Calving.-(See Milk Fever.)

Dysentery.-(See Consumption and Chronic Diarrhœa.)

Ectopia Cordis.-This is a deformity sometimes met with in calves at birth. The heart may be seen outside of the chest, or the lower portion of the neck, or even through an opening below the chest, and sometimes eren in the abdomen. The animal will have to be killed.

Epizootic Aphtha.-Murrain.-In some parts of the world, this disease is called murrain. This is one of the epizootic diseases of cattle, attacking the feet and mouth, and sometimes extending to the teats of the udder.

Causes.-Contagion is believed to be the cause, but I never can be reconciled to believe in this repeated bugbear, contagion as being the only cause of certain diseases. We all know, however, that when a disease is once established it can then effect others, but still we must consider that all diseases of whatever kind, must 
have had an origin without any contagious element to produce it. This, I firmly believe to be one of those diseases, depending not so much upon contagion, as upon what Sydenham would call the peculiar constitution of the year, exercising certain deleterious influences on the system, and soon followed by disease of some portion, or other of the body.

Symptoms.-Sore mouth, sore teats and sore feet, elevated vesicles within the mouth, on the teats, contain pus soon discharging and drying up by a scab forming. Fever and other constitutional symptoms now subside. When the feet are sorely affected, the sores burrow deep in between the hoof, and the sensitive structure of the foot, resulting in a complete separation of the hoof, and finally it is thrown off entirely.

It is painful to look, as I have done on several oceasions upon a whole row of cows suffering from suppuration, and falling off of the hoofs. The loss to the dairy men, consists in the loss of milk, and loss of condition in the cows; for if the cows are properly attended to, not any of them need die. The hoof will grow again, and be as useful as ever, from the fact, cows like pigs are not kept for racing purposes, so a second hoof although not so strong as the first one, will answer for the purpose of walking on soft ground, and gathering their food.

Treatment.-Apply to the sores the following lotion: sulphate of zinc, two drachms; water, one pint.

Prevention.-When once fairly established in a place, it is almost a certainty that all cows and neat cattle will take it, some developing it sooner than others; and to save time and expense, take the bull by the horns, and inoculate every one of them. Thus by producing the disease in this way, a week or so will see the last of it, and 
by good care not much time or loss will have been incurred, by so anticipating the disease. The milk of cows affected with this disease is poison.

CALves-By drinking the milk of cows with this disease, perish in great numbers.

Symptoms.-Difficulty in swallowing, cough and saliva driveling from the mouth. The whole accompanied by fever, and frequently ending in death.

Eye Diseases.-The cow is not so often the subject of disease of the eye, as horses are. There is however, one disease of the eye which cows are often affected with, namely : cancer of the eye, which can not be cured, but will ultimately destroy the eye altogether. For other diseases of the eye, see Eye Diseases in part first of the book.

\section{Falling Sickness.-(See Milk Fever.)}

Falling of the Womb,-Or Calf Bed-Reed, etc. This deviation from the normal or healthy condition, is a great trouble to the farmer and breeder.

Cause.-Relaxation of the horns or ligaments of the womb from a weak and relaxed habit of the body, accompanied by debility.

Prevention.-Immediately after calving, apply a truss or pad to the mouth of the vagina, and secure it in the following manner: put a large horse collar on the cow's neck; one surcingle round the body of the cow, behind the fore legs, and another in front of the udder and hind legs. This being done, attach a small soft rope to each side of the colar, and bring them along each side of the back bone, and give them a hitch round the fore surcingle, and the same on the hind one; then bring the ropes cluse together under the tail, and place the pad over the 
proper part, and the ropes laid firmly over it, and tie both ropes together with a string, just below the pad, then bring one rope down between the udder and thigh, and give it a hitch round the hind surcingle or band, and finally secure the end of the ropes to that portion coming along the back.

Treatment.-When the womb has fallen down and is inverted, assistance should be had at once. The womb or bag should be lifted into a clean cloth, and held up by a person on each side. There are two ways of returning the womb into its place, the one by pressure on the neck or small portion of the womb, and the other is by pressure to the fundus or large end, or bottom of the womb. This last is the best way, because we have not only the mere pulling of the womb to contend with, but it is inverted also. So therefore, apply gentle pressure to the bottom of the womb, first having cleaned it from dirt, dung and straw. The operation will be made more easy by having the cow placed with her fore legs low, and the hinds ones high, so that it will slip in without much difficulty. After it is in, the cow should be kept standing in such position, or even lying in this position, a day or so, and the pad already spoken of, should be applied as soon as the operation is finished. The pad is a much better way of securing the parts than by sewing the lips of the vagina.

Where all efforts fail to put back the bed, and when decomposition or mortification has ensued, the only chance to save the life of the cow is for to cut the womb or bed, at its smallest or neck portions; but before doing so, tie firmly round the neck of the womb a well waxed cord, which will prevent bleeding. This is to remain on the bed which is left. Give immediately, twenty-five drops 
of the tincture of aconite root every three hours, till four or five doses are given. Tnis will relieve pain, and control the circulation.

Fardel Bound.-This is a disease affecting the omasum, or third stomach of the cow or ox. (See Impaction of the Stomach and Hoven.)

Fever.-Cows are subject to ephemral fever, or more plainly speaking, a fever of a days duration, and passes of without any trouble. This fever has been thought by some persons, to depend upon hollow horn and wolf in the tail, and consequently have cut the tail, and bored the horns of the poor dumb brute.

Foul Claw.-Foul in the Foot.-This is a sore in between the digital spaces, and is caused by the animal standing in mud and moisture, which scalds the parts, and produces lameness.

Treatment.-Cleanse out the space with a hair rope or by some other means, and apply sulphate of zinc, one drachm; water, half a pint. Keep the feet dry and clean, which will not only assist the cure, but is a preventive also.

Gangrene of the Tail, or Gangrena Caudce Epizootica.-This is not, as yet at all events, an American disease, but is of frequent occurrence among the cows of continental Europe. This fact, however, I believe to be the only true reason why we in Pennsylvanla will persist that cows have wolves in the tail, accompanied with an empty horn.

Cause.-Unknown.

Symptoms.-We are told by Hering and Rychner that that the tail becomes paralyzed, its skin soft, swollen, and filled with water at its end, and when opened, a bad 
smelling ichor is discharged. The disease spreads upwards, where finally separation of the tail takes place, thus leaving the animal without one.

Garget Mammitis.-Inflammation of the udder in cows is often of a very troublesome character. It occurs shortly after calving, and in some instances not for a week or two after. The inflammation is usually confined to one quarter of the udder only, and may be of an acute, or chronic character.

Cause.-Want of proper attention in not milking the cow sufficiently after, and in many cases by not milking the cow when her udder is almost ready to burst, even before calving. In a word, if the udder, teats and milk ducts, are kept from over-gorgement, there can be no udder disease. The trouble is, that all cows are not alike in the prodnction of milk, but unfortunately they receive the same treatment; and hence, those cows which are endowed with great milking qualities, are usually the victims of garget.

Symptoms.-Heat, redness and pain, followed in a day or so by elevated swellings, which, if acute, will grow to a point, soft and fluctuating, and containing pus; and if not opener at this time, fistulous sores will be formed, which may cause final destruction of one portion of the udder.

When the heat and redness is not followed by swelling containing pus, this then is called chronic garget. In addition to those symptoms, the cow will have shivers and chills, not from cold, but from the muscular rigor which is set up in cases of extensive suppuration.

Treatment.-In the acute form, apply warm poultices to hasten suppuration or beeling; where the parts point, and contain fluid or pus, open deeply at the lowest 
point, that the pus may escape without forcing. When properly discharged, heal as for a common sore. See Ointments in Part II. of the book.) For chronic garget, cold application, so as to put it back, if possible; if not, hasten the suppurative process, and treat as for the acute form. Whatever treatment may be adopted, by all means do not forget to milk the udder severely, and a plan will be to put two strong calves to suck, for by so doing the pus may be drawn off by them.

Gastro-Enteritis in Calves.-(See Diarrhoa in Calves.)

Glossitis-Anthrax-Blain.-This is a disease affecting the tongue, and can only be taken for epizootic aphtha already described. Although apparently situated in the tongue, it is a constitutional disease-a blood passion in fact. This is also an epizootic disease, and as usual, it is thought to be contagious, or as much as to say, we know nothing of its cause, or mode of warfare. Few veterinary surgeons, or horse doctors, have, it would seem, ever known of zumins, ferments or leavens, as the Scriptures have it, for not in any work on veterinary science or animal husbandry are any of these words mentioned, nor a hint thrown out that such agents do exist, and have a deleterious effect upon the health of animals. I am satisfied that upon further inquiry and investigation, ferments will be found the only cause of such diseases, as are at present involved in darkness and obscurity.

Symptoms.-Loss of appetite, saliva flowing from the mouth, the tongue red, swollen, and inflamed, thus arersting mastication, or chewing: the muzzle or lips, head and neck swell, breathing disturbed. Appearances of suffocation set in, the saliva, is offensive to smell, and 
tinged with blood; the basis of the discharge is of a greenish color, not unlike vomicr seen in the lungs of glandered horses and consumptive men. In this condition, the animal will live from two to four days.

Treatment.-Lance the turgid tongue to relieve congestion, and wash the mouth with vinegar, and administer a slight purgative. One pound of epsom salts in two bottles of water, sweetened with molasses; a little powdered ginger may be added. Allow the animal plenty of cold water to drink, to cool the mouth and tongue, and allow pure air for the beast to breathe. Follow with two drachms, of the sulphate of iron, and an ounce each of ginger, fenugreek and sulphite of soda twice in the day, which will give strength to the body, and purity to the blood.

Framaturia.-Blood in the Urine.-This occurrence is not to be confounded with red water. Coagulated, or congealed blood comes when the first flow of water is discharged, and the remainder of the urine is clear.

Cause.-Blows or other injuries over the region of the kidneys, or from eating the leaves or tops of plants having a powerful diuretic effect, such as some of the yews, cedars and savins. Cows in calf, and mares with foal, will eat what other animals, or what they would not do themselves when in other condition. (See Red Water.)

Treatment.-Give linseed tea to drink, and slush mashes, and plenty of fluids to drink. No medicine will be required.

Hair Balls.-These are very common in cattle, and are introduced by the animal licking itself, and swallowing the hair. These balls are found after death, and are of various sizes, and thoroughly felted. 


\section{Herpes.-(See Mange.)}

Hide Bound.-This is not to be taken as a disease, but merely the result of faulty digestion and assimilation.

Treatment.-Give one pound of epsom salts, half an ounce of ginger, and mix in two bottles of cold water, and sweetened with molasses. Next day follow with the following powders:-Powdered ginger, one ounce; fenugreek, one ounce; carraway seeds, half an ounce: mix, and give in one dose, and one dose may be given daily for a week.

Hoose in Calves.-This is a common disease in breeding districts, and is very fatal in its results, and attacking young calves and cattle, till two years old. It is a parasitic disease.

Cause.-The presence of minute worms in the bronchial tubes. These worms are called filaria bronchi, and inhabit the windpipe of young cattle, sheep and lambs.

Prevention.-Keep calves, sheep and lambs on dry land, where there is no marsh, wet land or meadow.

Symptoms. — Constant, husky cough, difficulty in breathing, emaciation, and loss of appetite. Thus the disease goes on from bad to worse, until death takes place in from two to three weeks, depending much, however, upon the age of the beast.

Treatment.-Linseed oil, two ounces; oil or spirits of turpentine, half an ounce, well mixed with the linseed oil. This dose is for a calf of six months old. The dose is to be repeated every two days. Give the calves good feed, such as oil cake, etc. Another form, and it is a good one, and is generally used in sheep to save expense and trouble, is getting hold of them, and driving them into a pretty close house or shed, not larger than will hold all the affected ones. Then procure an earthern 
bowl or basin, containing one ounce each of common salt and oxide of manganese, and pour over this a mixture, say, water, half an ounce; sulphuric acid, one ounce and a half; stir with a stick, and chlorine gas will be evolved. When sufficiently stirred, leave the place, and close the door. Repeat the inhalations for two or three times, and let two days pass before each subsequent inhalation. If the animal be much weakened by the parasites, mix carraway and fenugreek in their feed, of each a quarter of an ounce, once a day for a week or so.

Hoven.-Tympanitis or Drum-belly.-So called from the appearance and sound. This disease is the evolution, or giving off of carbonic acid gas, from the large quantity of grass or clover when wet, contained within the rumen or paunch, together with the suspension of the function of digestion, and peristaltic action of the bowels, all of which combined, go to make up the disease called hoven. Hoven may occur in one hour, for we often see cows turned out to pasture in the morning, and are almost found suffocated in an hour afterward. There is obviously no time to be here lost. Every farmer should always be prepared to meet and cure such cases as they occur, as there is no time to run for assistance.

Cause.-Over filling the paunch, and in too quick a time, before the stomach has time to act upon it; and hence fermentation is set up.

Symptoms.-Great distress; the sides are distended, and when struck, sound like a drum, the beast lying and rising; the breathing is hurried; there is great suffering, and if not speedily relieved, the rumen will give way, burst or rupture; if this does not happen shortly, the brain becomes affected, and the beast dies unconscious. The disease runs its course with fearful rapidity. 
Treatment. - At once plunge a dinner knife, well sharpened, into the side, or at equal distance from the haunch bone and short rib, on the left side of the animal. Veterinary surgeons use a trocar for this purpose, and every farmer should have one also. If the case be not a severe one, it may be trusted to time, nature and medicine. Give half a pound of table mustard, and an ounce of the chloride of lime, mixed in as little cold water as will float the mustard out of the bottle, and down the throat of the animal. Or instead of the mustard and lime, give one ounce of recently powdered carbonate of ammonia in cold water. Dashing cold water over the loins of the beast often cure the affection, by inducing reflex action of the nervous centres of the body. Sometimes we see a chronic form of this complaint, occurring at intervals, owing to a debilitated condition of the walls of the rumen, and is cured by the following powder, given morning and night in the feed:-Powdered ginger, half an ounce; gentian, half an ounce; foenugreek, half an ounce: mix, and give in one dose, and continue them for a few days.

Hydatids in the Brain.-This is a parasitic disease, - a worm found floating in a serous fluid, surrounded by a sack or small bladder, and situated generally on one side of the brain, and under or near the base of the horn.

Cause.-This affection is caused by the animal eating with the feed or grass, some of the ova or eggs which have been dropped from dogs, affected with tape worms.

Symptoms. - The affected beast will be observed in the early season of the disease, to be affected in one of the eyes, ending shortly in total blindness of that organ. This, however, is not always the case. The great 
symptom chiefly to be relied upon, is the constant turning of the animal in the form of a circle. This, the animal will always persist in doing, and stop only when brought in contact with a stone wall or fence, which prevent further circular turning. Unfortunately, the poor animal comes in contact with the wall with such force, and so often repeated, that on that side of the head will be seen swellings and enlargements of the bones of the jaw. In this way does the affected beast turn the circuit from day to day until emaciated from weakness and hunger. The end of some cases is more short than this for not unfrequently they fall into holes, rivers, etc. The inability to stop turning prevents the animal from gathering food, and hence the case terminates in starvation.

Treatment.-The success attending the treatment of such cases is very great. The cure consists in finding out the exact spot on the head over the hydatid, and bore through the bone with an instrument made for that purpose. And as soon as the bone is bored through a small pair of forceps or tongs is put through the hole, and into the sack, and by this means together with a syringe with a long nozle to pump out what fluids there are in the cavity, complete the cure. The hole of course will have to be closed by a pad or cloth, to exclude the air till the hole have closed by a new bone.

Inflammation.-Inflammation is the same in all animals, but happily it is not common to the ox tribe. Indeed inflammation in all animals is not so common as it is used or, is represented to us to be. Irritation is more often observed among animals and men, than inflammation, and disease with decreased force or power is more frequent than either diseases now a days or, it 
may be that we are better educated; thereby can more readily discern the true conditions of diseases, than those of former times or, it may be from both causes combined, that we are able to state that we see such a change in diseases. However, the majority of diseases, now met with, are of a low type, and require for treatment not bleeding, but medicines and good feeding, to support the powers of nature.

Impaction of the Omasum:-(Or third Stomach.) In some parts of the world this disease is called "wood evil." So called because the stomach is filled with leaves, dried bramble and sticks or twigs, from bushes. These articles being deficient in nutritive matter, cause the suspension of rumination (chewing the cud,) and digestion.

Causes.-As already hinted, it is caused by, and in addition to the above, dried grass, which has been left standing in the field, or in fact any kind of food, which does not contain essential principles for fat and blood making, in proportion to its bulk, is sure to bring on an attack of wood evil or impaction of the third stomach.

Symptoms.-Loss of cud, loss of appetite, quickened breathing and accompanied with a grunt. Diarrhœa is followed by constipation, great thirst, the legs, horns and ears are cold, grinding of the teeth, and when the disease has about run its course, moaning takes the place of the grunt, and diarrhoea now succeeds costiveness, and the poor beast dies exhausted. Examinations after death disclose a curious condition, namely: the third stomach is perfectly stuffed full with food, and it is so hard and dry, that it readily burns when fire is applied to it.

Treatment.-Allow the animal plenty of cold water 
to drink, so that its mechanical effects may be taken advantage of by assisting to wash, dilute and moisten the dry mass. Give strong purgatives. Epsom salts, one pound; table salt, half a pound; oil of croton, fifteen drops; mix, and give in one dose, in fully a gallon of water, for be it remembered that cattle should have all medicines given in large fluids, and it is more imperatively demanded in cases like this, where so much depends upon fluids. If twenty-four hours pass by without any effect from the salts, repeat the same dose with an addition of two ounces of the spirits of turpentine. Purgative medicine will now be stopped here, whether they have been of any effect or not. So long as the animal does not seem stupid or the brain be not affected, there are still hopes that recovery may reward our labors.

Jaundice.-This is a common disease in the ox, from the fact he is supplied with a gall bladder, and gall in great quantity. Jaundice may be properly called biliary intoxication, or distribution of bile throughout the whole circulation of the body.

Causes.-Closure of the biliary ducts in the liver, and the consequent absorption of the bile into the stomach. The bile duct may be closed from gall stones.

Symptoms.-In white-skin oxen, jaundice is seen at once from their yellow-colored skin. In dark colored animals we will be satisfied to examine the lining of the mouth, nose and eyes, for this yellow appearance. In addition to these signs, we have dullness, costiveness, and the dung is of a whitish or straw colored look. If the symptoms be not very prominent, the animal may be left with safety, to the powers of nature, which will be assisted by giving slop food, or placing the animal upon bare pasture for a few days. If the case be more of an 
acute kind, give a dose of purgative medicine. Epsom salts, one pound; table salt, half a pound; ginger, half an ounce; mix, and dissolve in four bottles of water, sweetened with molasses.

Kidney Disease.-Kidney disease in the ox, is of a rare occurrence. Disease of the kidneys is the disease of animals, whose lives are allowed to run longer than those of oxen, especially when fat and fit for market.

\section{Laryngitis.-(See Croup and Bronchitis.)}

Leucorrhœa.-A discharge of muco-purulent matter from the womb and vagina of cows.

Cause.-Debility and bloodless condition of the system.

Treatment.-Iron, gentian, fenugreec, the mineral acids and good nurishing food are what is wanted to build up the system, and a weak solution of lime may be injected into the vagina once a day. Give the following powder morning and night, powdered sulphate of iron, two drachms; powdered gentian root, half an ounce; powdered ginger, half an ounce; fenugreec, half an ounce: $\mathrm{mix}$, and give in one dose, and continue them for a week. Commercial sulphuric acid in half ounce doses by weight, may be given once a day in half a bucket of cold water, which the cow will readily drink.

Lice.-(See Lice in Part I. of the book.)

Liver Disease.-(See Jaundice.

Locked-Jaw.-Kill the beast and dress it for market as soon as it is known that it is locked-jawed.

Lung Diseases.-(See Pleuro-pneumonia.)

Lymphangitis. - An affection of the ox tribe, following an accident, and partakes of some of the characteris- 
tics attending upon farcy in horses, but without any specific poison being developed.

Treatment.-A dose of salts internally and formentations or poultices apply to the swelling and to the sores if there be any, which will depend whether the skin gives way or not.

\section{Malignant Catarrh.-(See Catarrhus, etc.)}

\section{Mammitis.-(See Garget.)}

\section{Mange.-(See Mange in Part I of the book.)}

Milk Fever.-Febris Lactea.-Milk fever occurs from the first to the third day after calving. Rarely after the third day, and is seldom met with before the fourth calving, and then attacking chiefly cows of select breeds, and good milkers. Milk fever consists in inflammation of the womb, and sometimes even extending to the bowels.

Symptoms.-Loss of appetite, chewing the cud, or rumination ceases, staggering gait, wild look, falls and cannot rise; and if the disease be not checked the brain will soon be affected also, then the cow will dash about with her head and horns plunging them into the ground.

Cause.-Undue determination of the blood to the womb from over feeding before, and immediately after calving, and from sudden changes of the weather at the time of calving.

Prevention.-Give one week before calving, one pound of epsum salts, half a pound of table salt, and half an ounce of ground ginger mixed in four bottles of cold water, and sweetened with molasses. Let the cow's feed be of the lightest kind, such as hay and thin slop mashes, and no meal, grain or solid food. This measure will lessen the tendency to interruption of the circulation, and will 
improve the health and tone of the whole system. And to avoid as much as possible, the effects of sudden changes of the weather, have the cow brought into the house. And when milk fever is anticipated, give a few hours after calving, twenty-five drops of the tincture of aconite root, and may be repeated every six hours till four doses have been given. Nothing that I am acquainted with is capable of equalizing the circulation of the blood by controlling the heart's action, and thereby the circula. tion like aconite, and for this purpose I highly roommend it to farmers and breeders of stock throughout the country. These measures, together with light slop feeding for a few days after calving, I am sure will prevent much suffering to the cow, and inconvenience and loss to the owner.

Treatment.-When the disease is present, give at once thirty drops of the tincture of aconite root, and half an ounce of the pure opium in powder, and give it in a bottle of thin gruel. The aconite to be repeated every four hours without the opium; place chopped ice in a bag on the fore head, and attach it to the horns, and renew the ice when it is wanted. This being done quickly, at more leisure get and give epsom salts, one pound; table salt, one pound; ginger, half an ounce; mix and dissolve in four buttles of cold water with a little molasses to sweeten it, and give at one dose. After this medicine has been given, turn the cow from side to side every four hours, or when the aconite is given, this will save labor and unnecessary excitement to the cow, which should be left as quiet as possible, keep the legs and body warm, thereby relieving the womb to that extent. Do not deny pure air, nor plenty of cold water to the afflicted animal, for she not only needs them, but they are indispensible to a sure and 
perfect recovery in most diseases, and as much so if not more in a disease of this kind.

Milk Trembles.-This is a disease seen in the wooded country of the South and South-west, and depends upon cold, moisture and miasma. The disease disappears with improvements and clearing of the land from timber.

Treatment.-Give gentian, ginger, and fenugreec, of each, half an ounce; mix, and make one dose, which may be repeated once in the day.

\section{Moor Evil.-(See Red Water.) .}

\section{Mouth Discase.-(See Epizootic Aphtha.)}

Murrain.-This is one of those names given to diseases of cattle which ought long ago to have been expunged from the books and writings of men who ought if they do not know that the word murrain means to die. By classical scholars, orators, and poets the word, murrain may be taken as an indication that they have read Virgil, Homer and Horace; butwhen cow doctors talk about the murrain, it conveys the reverse idea we entertain about the orator and poet. Cattle plagues and murrain are excellent names, whereby the ignorant pretender gains credit among farmers and others, for a knowledge he never did possess. The name murrain may then be given to any disease or diseases, however different the one may be from the other in sign, symptom and seat, providing that the beast die. It will be observed, however, that if the animal should live, murrain can't or could not be its right or proper name. Murrain as applied to cattle diseases conveys no idea of the nature or seat of the disease, and may be given to any disease a man may choose. In some parts of the world, murrain is applied to epizootic aphtha, a disease affecting the mouth and feet, and withal, not a deadly 
or fatal disease. Without fatality, a person would naturally and knowingly think that there can be no murrain. The absurdity of the name so applied, can induceno very high opinion of cow doctors in the minds of intelligent men.

Nephritis.-This is a name given to disease, or inflammation of the kidneys. Scarcely ever seen in the ox.

Nervous Diseases.-These may be named as follows:-Brain diseases, apoplexy, inflammation of the brain, palsy, paralysis, locked jaw, twitching of the muscles, etc.

CEstromania.-A term applied to cows when bulling.

Estrus Bovis.-The fly which deposits the eggs, from which the ox bot is developed.

Osseous.-Composed of bone, bany tumor.

Ovarian Dropsy.-This is a disease peculiar to milch cows, and consists of watery swellings of the ovary, usually one ovary being affected. Ovarian dropsy is characterized by a large, soft swelling, situated upon the side of the cow, between the short rib and the thigh.

Treatment.-Tapping the tumor with an instrument called a trocar, whereby the fluid is let out: when this is done, feed the cow well, and give her iron, gentian and ginger, to prevent further accumulation.

Ovarian Tumor.-The difference between ovarian dropsy and ovarian tumor, as ascertained by the touch, is, the dropsy is soft and fluctuating, and the tumor hard and slightly movable. Ovarian tumors are generally hard, and similar to cancroid and encephaloid tumors found in man and animals. 
Treatment.-Fatten the animal, kill and send her to market, before she dies, and be a complete loss.

Palsy, or Paralysis.-This is a nervous disease, and is characterized by the animal having lost the power of standing. Palsy may be partial or complete.

Cause.-Tumors on the brain, injury to, or softening of the spinal cord.

Symptoms.-Inability of the animal to move, continued laying, will usually eat and drink as if nothing were amiss, and will continue to do so for many weeks.

Treatment.-We are in possession of only ont drug, which is oftentimes of service in curing this disease, depending on whether the paralysis be from reflex action of the nerves of motion, or from entire suspension of feeling. If the case be from reflex action, then this medicine had better not be given; but if the nervous feeling be entirely lost, the strychnia may then be given in one grain doses twice in the day in the animal's feed, and continue it for a week or two.

\section{Parasitic Lung Disease.-(See Hoose.)}

Parturient Fever.-(See Milk Fever.)

Phthisis.-(See Consumption and Diarrhœa.)

Placenta.-This is a name given to the after-birth.

Plethora.-Fatness-full of blood.

Pleurisy.-This is inflammation of the peuro, or white, fibrous, serous tissue lining the ribs within the chest, and the covering of the lungs. For further particulars we will introduce them in the next article.

Pleuro-pneumonia.-“Massachusetts Cattle Disease," or Epizootic Pleuro-pneumonia.-Pleuropneumonia is a compound disease, as its name indicates- 
pleuro and pneumonia. My own opinion is, however, and it is almost a certainty, I think, that the single name pleurisy would cover the whole ground, for the pathology of the disease unmistakably points to this one fact, that the lungs are not affected as a disease from the beginning, but the solid, spotted and mottled condition of the lungs are but the effects of disease of the pleural covering, and consequent effusions of serum in to the chest, floating and surrounding the lungs, together with weakness, low vitality, and debility of the animal affected. This then is the reason why the lungs have become diseased, and the consequent double name given to the affection.

However much men may doubt it, this diseases is nothing more than buccal inflammation, extending from the coverings of the mouth and nose down to the pleural membranes within the chest, speedily followed by extensive outpouring of fluid called serum, and it is not till this fluid has accumulated in sufficient quantity that the evidences of disease show itself to the farmer or breeder. Cows are not so nervous, nor yet so excitable as horses are, whether in health or sickness, and hence, the absence of those symptoms in the early stage of the disease in cows, which are so early shown in horses, when affected by the same sickness. - Farmers, remember this, for in early detection lies often the life of the animal.

Again, and in support of my theory, if the lungs were one of the primary seats of the disease, it would not be reasonable to expect the animal to live for a week, and in some cases a month, as is the case with animals affected with this disease. In no disease of the lungs, excepting tubercles, will animals live so long as those do when affected with pleuro-pneumonia, thus entirely disproving the universal theory of all and every person who has an idea to offer upon the subject. 
The length of time animals live with this disease, together with the serum, the condition of the lungs, and the adhesions so often seen connecting the lungs with the sides of the chest, does not go to prove the incurability of the disease at all, as has been so often asserted, but on the contrary it goes to show the curability of the disease, and the ignorance of those who have been guilty of so bold and unwarranted an assertion, thereby deceiving the honest cow keeper and breeder to their great loss, and in some cases to their ruin. Pleuro-pneumonia then I take to be a disease similar to typhoid influenza in the horse, affecting the white membranes of the nose, mouth, wind pipe and chest, speedily followed by outpouring of fluid into the cavity of the thorax or chest, gradually increasing in volumn, till the lungs are nearly submerged, solidifying them, thus arresting areation, or proper oxygenation of the blood, together with shreds of plastic lymph connecting and interfering still further with the action of whatever healthy lung there may be left: thus by a gradual, but progressive process, the vital power gives way, and the poor beast dies from suffocation, or asphyxia, in from one week to two months or more, depending if the cow be in calf, and what condition otherwise the animal may be in. The better the condition, the longer will they live, and the more likely are they to live, even without medicine, thus giving the lie to its incurability. (See Bronchitis.)

Cause.-Some subtle poison in the atmosphere sometimes, and not always present, sudden and severe changes in the temperature, cold, heat, dryness and moisture, easterly winds, and possibly some other conditions which may be present, but not recognized, and give rise to what is called the predisposing cause, for certainly we have those 
various changes in the condition of the weather, without producing the disease, something being wanted in the animal economy to act as a predisposing cause, thus two causes being necessary to produce epizootic disease, namely: the predisposing cause which resides in the system, and the exciting cause, which belongs to the atmosphere. Atmospheric cause is being clearly proven from the fact of the early symptoms of the disease being irritation of the mucous membranes of the nostrils, nose, eyes, etc. That this may be properly understood, let any person enter the too frequently over heated lecture room or theatre, with its impure air, and on coming out to the keen air of night, how the membranes of the eyes drop tears, and sneezing from irritation of the nose takes place. Pleuro-pneumonia being a disease chiefly attacking milch cows and working oxen, rarely affecting herds of cattle in the field; thus we are carried back again in our inquiries to the cow house, barn yard and its surroundings-the slop feed stimulating the cow to over secretion of milk, and at the expense of her general health and condition-the smoking and putrefying dung heap-the imperfect ventilation and over-heated stablegiving of stimulating feed, and immediately after turning the heated cow out to the cold, and sometimes frozen watering trough, to quench her thirst. In one or other of these anomalies or all combined, will be found the cause of this epizootic disease. Contagion, if it is really contagious, which I honestly doubt and even deny, however, if it is contagion then it cannot be the only exciting and predisposing cause of the disease.

Symptoms.-As has been already stated, the early symptoms are irritation of the membranes of the nose, windpipe, etc. The symptoms of this irritation are not 
perceived by the farmer, till effusions of fluid are poured into the chest in considerable quantities, and interfering with the movements of the lungs, and consequently the breathing or respiration then-and then only does the farmer observe that the animal is sick. The presence of irritation in the windpipe can be detected by placing the ear close to the wind-pipe, previously tightening the skin upon it, when a slight grating sound is heard. The immediate effects of irritation of the wind-pipe of the cow, is a slight discharge of an acrid fluid, of the appearance of water, from the corners of the eyes and nose, which is, however, sometimes so slight that ordinary persons fail to see it, and if they did-would attach no importance to it, and so the first, and most important time and symptoms is passed by uncared for, unheeded and unchecked. The next and important symptom is a half involuntary cough, or rather a husking sound, not the clear cough which horses give, when similarly affected. When cough is heard, it may then be said the incubative stage has passed away. I have said incubative, although not believing in such an existence, if it be not in the passive condition of the minds of men, who give credence to such a theory. Milk is now small in quantity, thin and blue, cough more loud and oftener, breathing quick and laboured, accompanied with a grunt, rumination is irregular, and the appetite precarious. Such then are the sypmptoms that may be seen by the ordinary observer. Auscultation and percussion applied to the sides of the chest, are not to be relied upon by persons who are not accustomed to the chest murmurs, or sounds in health and diseases. Animals in good condition rally from the attack and get well, without assistance from medicine or art. In weak and low conditioned animals, 
the disease and symptoms are gradual and progressive, and finally the animal dies in from two weeks to two months.

Treatment.-The success of the treatment here reccommended, will depend, 1st. Upon whether the animal be in good or low condition; 2nd. Whether the cow be heavy with calf; $3 \mathrm{~d}$. Whether the disease has been discovered, and treatment applied in good season. These are important inquiries, for much depends upon them, for this disease is much more frequent and fatal in cows heavy with calf, and animals in low condition. If the disease has been observed within forty eight hours from the time of attack, give the following powders every four hours between six o'clock in the morning and at ten at night, or at six, ten, two, six and ten o'clock:

Tincture of aconite root, two drachms and a half; powdered gentian ront, three ounces; powdered ginger root, three ounces; sulphate of iron, two ounces: mix well, and divide into five powders and give as above directed. After the five powders have been given, continue with the same powders, but without the aconite, and give them only three times in the day, instead of five as above directed. Half an ounce of the sulphite of soda may be added to each powder with advantage. Give occasionally once a day, half an ounce of oil of vitriol, or sulphuric acid by weight, and mixed in half a bucket of cold water, which the animal will readily drink. The powder will have to be mixed in a large bottle of water, and sweetened with molasses. Allow plenty of pure air, cold water and good strong feed, but not too much at a time; this will be better than thin, poor and non-strengthening slop, which is so injurious and deceptive, and so often recommended and adopted in cases of sickness. In the early stages of the disease, the carbo- 
nate of ammonia given in three drachm doses, along with the other powders, will do much good. There are two points I have ever sought to obtain, in the treatment of this disease. 1st, To mantain the appetite. 2nd, To restore and maintain it, if it be lost.

Prolapsus Vaginæ.-Procidenta.-This is a protrusion of the womb through the vulva, and is an accident of frequent occurrence with cows when accumulating flesh fast, and also among cows, denominated bullers. Sometimes, however, it may be due to tumors, or dropsy in the womb or ovaries.

Treatment.-If from ovarian disease, spay the cow, and in simple cases elevate the hind parts of the cow, by making the stall lower at front and higher behind, and place a pad on the parts, after the manner described, under the article Falling of the Womb.

\section{Prolapsus Vesicœ.-(See Bladder Inversion of.)}

Prurigo Vernalis.-This is a disease of the Spring of the year, attacking cattle, and characterized by extreme itchness-the body is covered with inflammatory spots, which discharge pus, and form scabs, which fall off and leave the parts baid, or without hair.

Treatment.-Give a dose of epsom salts, and fumigate the beast with sulphurous acid gas. (See Sulphurous acid gas, in Part II. of the book.) Give good feeding and good shelter.

\section{Puerpural Fever.-(See Milk Fever.)}

\section{Quarter Evil.-(See Black Leg.)}

Red Water.- This is a disease of milch cows soon after calving, and consists in an altered condition of the blood in which the red globules are broken up, and the 
coloring matter which is called hematosin escapes and is passed with the urine.

Cause.-Obscure, but assigned to local causes as the peculiar properties of certain herbs or grasses which the cow feeds upon. My opinion, however, is that the cause will be found to be local congestion-the red coloring matter is but the product-red colored serum.

Symptoms.-Diarrhœa lasting for a day or two, followed by constipation, urine of a brown color changing to a deep red, and in the last and fatal stages the urine is black, resembling porter.

Post Mortem-Appearances confirm my opinion that it is local congestion that gives rise to red water. The liver and kidney being chiefly implicated.

Treatment.-Give a strong dose of epsom and common table salt, one pound each, and half an ounce of ginger; dissolved in four bottles of water and sweetened with molasses. Give mashes pretty well wet and in a day or two follow by giving powdered ginger root, half an ounce; powdered gentian root, half an ounce; powdered fenugreec, half an ounce; mix and make one dose, give one dose twice in the day, till sufficient improvement takes place to warrant no further medicine to be given. To hasten recovery, give good and generous feeding, which will also assist in making blood for that which has been lost.

Reticulem.-The second stomach so called from ret or net like, and is sometimes called the honeycomb.

Retroflexion of the Womb.-Retroflexion is said to be present when the canal is bent on itself.

Retroversion of the Womb.-This term is applied when the canal is straight. 
Rheumatism.-(See Rheumatism in Part I. of the book)

Rinderpest.-This is the Dutch name for Cattle Plague. So much am I against such ignorant and unmeaning names being used by persons you would insult, if they but only knew that you had but a poor opinion of their scientific attainments. The disease will be found treated of under the article Typhus Contagiosus Boum, (contagious typhus of cattle.)

Ringworm.-This is a parasitic disease, and consists in the growth of cellular tumors on the skin. Young animals of one and two years are most subject to the attack of the parsasites.

Symptoms.-Broad and flattered elevations on the skin.

Treatment.-If left to themselves they will die out in a year. The oxide of zinc ointment will speedily cure the affection. It is considered a contagious disease, although not a fatal one.-(See Ointments in Part II. of the book.)

Rot.-(See Diarrhœa and Consumption.)

Rumen or Paunch.-This is the first stomach and is the receptacle for all food that is gathered, and is kept there till wanted, or till the rumen is full, when rumination is usually begun by contraction of the rumen upon its contents, whereby portions of the food are forced into the gullet and mouth, to be remasticated, and finally passes down the gullet again this time, not over the floor of the canal, but it separates the pillars, and passes not into the rumen, but into the manyplus, or third stomach.

Rupture in Calves.-The rupture we are now considering is what is known by the name Umbilical, which occurs in young colts and calves, and consists in the protrusion of a portion of the bowel and intestine through 
the navel, thus forming a small tumor. This condition is often congenital, or found on the calf at birth.

Treatment.-Force the bowel up into the belly and gather the loose skin together, and tie a well waxed cord tightly round it close to the belly, and a strong pin may be forced through the skin below the ligature or cord, to keep it from falling off before the loose skin comes away. In a few days the skin will fall off, leaving a healthy sore without any hole or rupture.

Another form of treatment is to place a bandage round the body, and a pad over the rupture, as is racommended for the same condition in colts, which see. Whatever plan that may be adopted for the cure of umbilical hernia in calves, each one must be kept separate from one another, as each will suck the other, thus preventing closure of the rupture and healing of the parts.

Siberian Boil Plague.-Carbuncular Disease.This is one of those diseases affecting cattle on the Russian Siberian Steppes. It is a blood poison or pyemia, and is closely allied to the other Russian Steppe disease, commonly called rinderpest and cattle plague.

\section{Slinking the Calf.-(See Abortion.)}

\section{Sore Throat.-(See Bronchitis.)}

Spaying. - This is the name given to an operation for the removal of the ovaries or female testicles from the milch cow and young female oxen. The object for the removal of the ovaries from young cows that never have had a calf, is to prevent them from ever having a desire for the male, and that she will be the more easily fatiened and fitted for the market. Young cows so operated upon, are henceforth called heifers. 
Spayed Cows, the Advantages of- - The following are the reasons why dairy men should spay their cows, when not intended for breeding:

1. Spayed cows are more easily kept in good condition than cows not spayed.

2. They are less liable to sickness of an epizootic kind, and when sick, more certain and easy of cure.

3. When epizootic diseases are present in the vicinity, or even in the herd, spayed cows are always in condition, and fit for the butcher, and to prevent loss and save expense in the treatment, with the attendant risk of loss of some, and loss of condition and milk of all that are affected, they can be sold not at a loss as is the case with cows not spayed, and when pleuro-pneumonia is among them.

4. Spayed cows give the same quantity and quality of milk all the year round, if they are properly fed and cared for.

5. Ten spayed cows will give the year round as much milk as double the number of cows not spayed, thus saving the interest on the outlay for ten cows, together with the absence of risk from loss of some of the principal by death of one or more from sickness, or accident, not to speak of the feed of ten cows. The feed of ten cows and the manure of ten cows, the farmer can best tell the difference in their value.

6. With spayed cows there is no risk to run from milk fever, nor troubled with cows called bullers.

7. To fatten a cow, spay her and not give her the bull, as is the present custom by which feed and time are consumed, and yet the animal still is not very fat after all for she has to provide fattening substance to the calf in the womb, whereas if she had been spayed, would have been appropriated to herself; nor is this all, for the calf 
in the belly of the cow is at once discounted by the butcher, as it is not a saleable article in market.

8. Spayed cows cannot abort or slink their calves. We have now had a bird's eye view of the advantages to be desired from spayed cows, now let us look in the same manner at the disadvantages of spayed ones.

1. The expense of the operation and attendant risk of the animal dying, although this is not great, about one in the hundred, and the expense of the operation will be from three to five dollars, which will depend upon the distance the operator has to travel, and how many animals are to be operated upon.

2. Spayed cows are apt to accumulate fat and flesh so that they will become dry much sooner than cows not spayed, still there can be little loss, for a fat cow is always ready for sale. These then are the objections to the spaying of cows, if objections they may be called. We now leave the subject to those who are more immediately interested.

Spleenic Apoplexy.-This is a new disease, and consists in engorgement of the spleen with blood, and subsequent rupture of the organ, followed by death in from twelve to twenty-four hours from the time of atttack.

Cause.-This disease is clearly traceable to the new, and what is wrongfully called improved feeding and fattening of cattle. Food, rich in nutritive qualities, and deficient in fluid's is the great cause of spleenic apoplexy in cattle.

Symptoms.-Constitutional disturbance is set up all at once, and no early symptoms manifested. The animal is well to every appearance at one time in the day and dead by evening. When the symptoms are seen, they are as follows: Colicky pains, twitching of the muscles, stagger- 
ing gait, frothing from the mouth, the urine colored and mixed with blood, the animal falls and dies. The immediate cause of death being coma, from derangement of the brain.

Treatment.-Treat the case by way of prevention with low diet. No treatment however well directed, is of any use when the disease has once manifested itself. Life is prolonged a few hours by blood letting, but no cure can be effected.

Spring Eruption.-(See Ringworm.)

Stomach Staggers.-(See Hoven.)

Strangulation.-(See Choking.)

Sturdy.-(See Hydatides.)

Teats.-Teats of milch cows are often subject to diseases and derangement.

First. Milk stones sometimes stop the flow of milk, and are felt within the milk channel, when the teat is pressed between the fingers. For their removal, take a silver probe or knitting needle, and if possible, force the obstruction up into the udder.

Second. Strictures of the milk channel of the teat, which cause a small stream of milk to flow, and impair the usefulness of that quarter of the udder. This can also be remedied by using a silver probe or needle, commencing, however, with a small size, and gradually using thicker or larger ones, till the channel is as large as wanted. This operation will have to be carried on for a week or two, the first day using the small probe three times in the day, and following the next day with the larger size, and so continue with other sizes, till finally cured.

Third. Warts are of occasional trouble to the cow, and to those who milk her. The irritation, and some- 
times swelling is induced, which cause closure in the channel of the teat. Warts are to be removed by a pair of sharp scissors.

Fourth. Sore teats are cured by the simple ointment, one ounce, and one drachm of the rust of copper, coinmonly called verdigris, added to it.

Teeth Diseased.-Diseases of the teeth of cattle are not so common as in horses. However, I have seen cases of a diseased tooth in cows, causing a bony enlargement on the lower jaw, and subsequent fistulous opening, and discharges running from it. When disease of the roots of the upper teeth takes place, it is accompanied with a bad smelling discharge from the nostril on the side on which the diseased tooth is.

Texan Fever.-This is a disease accompanied with sympathetic fever, and is not confined to Texas alone, for we see it more or less every year in the Eastern and Middle States, showing itself at the close of the summer months to the end of autumn. Texan fever is nothing more than the Fardle Bound of the European writersimpaction of the manyplus with withered and dried grass and herbage containing no moisture whatever: the stomach refuses to digest it. Heat, dryness and fever of the system is thus set up, with all their attendant consequences.

Treatment.-Large doses of epsom or glauber salts, dissolved in great quantities of molasses water. If no relief follows in twenty-four hours, repeat the dose again, bearing in mind all the while that great quantities of fluid or cold water is a means to overcome the dry condition of the impacted stomach. Indeed, the cure has a good deal of a mechanical nature about it, for by large drenches of water with the salts, do not only assist their 
action, but in many cases washes and dissolves the dry feed into a soft mass or pulp which will readily pass away, and the poor beast be relieved from pain, and is cured. Suspect this disease when occurring after a good grass growing spring, succeed by a dry, scorching summer, converting grass into spindles containing no moisture, and little nutritive properties.

\section{Throat Diseases.-(See Bronchitis.)}

\section{Thrush.-(See Epizootic Aphtha.)}

Tongue of the $\mathbf{0 x}$.-We speak of the tongue of the ox not with the view of its diseases, for that has already been done when speaking of aphtha; but for the purpesa of explaining the difference in the appearance between the tongue of the horse and that of the ox; for occasionally they are seen side by side in provision stores and other places, and sold as the tongue of the ox or cow. The appearance of a salted tongue of the horse is much shorter and broader at its tip, and is, withal, the finest looking; and to those who are not aware of the nature of things, they are most likely to prefer and purchase the horse tongue to that of the ox. The ox tongue is long, and narrow at its point. The ox can flex and use its tongue much the same as a cat can handle her phrensile paw. The horse cannot do this, but gathers his food with his front teeth: the ox gathers his with his tongue. Avoid the good-looking, broad and short tongue, and choose the long, narrow and irregular one; then you may be sure of the tongue of the ox.

Tubercle Disease.-(See Consumption and Diarrhœe.)

Tympanitis.-(See Hoven.) 


\section{Typhus Contagiosus Boun-Contagious Ty-} phus of Cattle-Rinderpest-Cattle PlagueSteppe Disease-Loserdurre, etc.-This is one of those epizootic diseases which have, at distant and different periods of the world's history, attacked the ox tribe of Europe, and consists in inflammation and irritation of the fibrous, serous tissue, or white membranes of the nose, windpipe and chest, and instead of serum or fluid being poured out as a consequence, as it does in cases of epizootic pleuro-pneumonia, the membranes become deteriorated, and portions become detached, and some partially adhere, but all decay, and become a dangerous poison, which is gradually absorbed into the general circulation, speedily followed by fermentation of the blood within the body, resulting in boils, or small carbuncles containing pus, which soon break and discharge. All of which is accompanied by sympathetic fever, but typhus so called, gradual and progressive in its character, till the blood and tissue of the body are no longer fit for the purposes of life, the animal dying, an exhausted and miserable object, in from a few days to one, two and three weeks from the time of attack.

Causes.-Certair conditions of the air and earth, as heat and moisture, cold and dryness, contagion. These may be called the exciting causes; something still being wanted in the system of the animal to form the predisposing cause, as debility, and a low standard of general health. Indeed, those conditions which sometimes exist, and form pleuro-pneumonia, are capable of producing contagious typhus. I am borne out in this opinion by Jessen, who among the discordant opinions and theories of Europe, has declared the disease to be associated with pleuro-pneumonia; and Dr. Greenhow says that conta- 
gious typhus existed side by side with pulmonary disease in England, in the middle of the last century. Why theorize any further, for it is so plain that those who run can read, that veterinary surgeons, politicians and notority hunters have, at the expense of the suffering farmers of Europe, continued to perplex and puzzle too long? The same has been attempted in Pennsylvania, and other States of the Union, even in cases of simple sporadic pleuro-pneumonia in milch cows, whereby, with a metmorphosis far outstripping those of Publius Ovidius Naso, the one disease was, as if by the wand of the magician, converted at once into that of another.

Symptoms. - We are told that the disease has its period of incubation, varying from four to fourteen days. This is simply nonsense; where is the repeated incubation in cows when affected with pleuro-pneumonia? No where, but in the minds of men who know nothing of pathology. Is incubation to be taken for blindness, and ignorance for science and education? It may be asserted, with equal truth and justice, that the condemned culprit, or rather suspended animation, has its period of incubation from the time of his condemnation to death, till the very moment for preparation for eternity, however long that may be. The weeping eye, the acrid drop from the inner corner of the nostrils, together with slight rough sounds from the wind-pipe, will tell the intelligent and scientific observer that something is wrong, and all this can be told from twelve to twenty-four hours from the time of attack. Where, then, is the period of incubation? The echo answers where! The husky cough, with dullness and prostration, follow the weeping eye and nostril. The back is arched, appetite and rumiation irregular, the rigor and the chill will tell that blood poison is present, and suppuration is 
going on as a remedial and natural measure, to get rid of the foreign or poisonous matter which is in the blood. The breathing is now labored and the heat of the body is variable, the skin and hair are now looking unhealthy, and fever is high. If the mouth and nose be examined eruptions and sores will be seen, and these sometimes extend to the feet. All of those symptoms are now speedily followed by discharges from the nose and eyes, of pus streaked with blood. Salivation and diarrhœa supervene, together with stupor, and with all the phenomena of approaching death. Cows heavy in calf and other debilitated animals as in this disease, and so with pleuro-pneumonia die sooner, and do not bear up under it so well as animals in better condition. The intelligent and careful reader cannot fail to see several prominent and important symptoms common alike to this disease and epizootic pleuro-pnenmonia, which see.

Treatment.-The indications of treatment are at once apparentfrom the nature, symptoms and post-mortem,or appearances after death. All taken somatically and considered, amount to this, namely, the animal is affected with fermentation of the blood, consequently upon inflammation and deterioration of the fibrous serous tissue of the body, accompanied with fever of a typhus type. Then the first thing to be done is, to build up the system and arrest fermentation by the elimination or neutralization of the Zumin, leaven, or ferment. For this purpose then, we will order the following powder: Powdered carbonate of ammonia, one ounce; powdered sulphate of iron or copper, three drachms; powdered gentian root, half an ounce; powdered ginger root, half an ounce; mix and make one dose, and give one dose of the powder morning and night. In the middle of the day give one ounce of sulphite of 
soda daily. Commercial sulphuric acid to be given in half ounce doses by weight, once to twice in the day; in a half bucket of cold water. The animal to be fed well and generously, but not by any means to arrest digestion by over feeding. Such then is about the plan I would recommend for the cure of contagious typhus in cattle; I would add however, or take from, just as the indications appeared. Thus I would have them daily inhale for an hour sulphurous acid gas. For the manner of using it, see Part II. of the book.

The treatment here offered will cure a major part of all affected. The number will depend upon the condition of the animals, whether they are cows and with calf, or are debilitated animals, etc.

English Treatment.-Professor Gamgee, the great veterinarian says, little can be done beyond purging the animal, etc., and says all treatment appears futile. The word "appears" Mr. Gamgee, implies a doubt; scientific men should be more emphatic and particular in the words used, and further, it conveys the idea in my mind, that he speaks of the experience of others, and not of himself. If there is any one remedy to be avoided in this disease of cattle it is purging or physicing. Will a dose of physic cure blood poison, build up the system, and arrest typhus? The person who says so is not fit to give an opinion on the subject. Out of many evils, choose that which is the least; that is, restore the digestive functions with carminitives and tonics, and not physic; remove the cause of the derangement, and the effects will cease, and do not take the bull by the horns, or beard the lion in his den. A Mr. Dobson devotes nearly ten pages to the subject, and winds up with the following words: "We trust we have said enough to give our readers a fair idea of the nature and 
consequence of the Rinderpest." Mr. Dobson has not, as he thinks he has, given either the true nature or pathology of the disease; and has not gone so far as Mr. Gamgee, for he has not even hinted so much, as there is a dose of physic in existence. In so far as this article of his is concerned, he has proved one thing-that a man can write much about what he really does not understand. The treatment, according to Mr. Moor, the veterinary homeopathist, is nearer the kind of treatment than any English author $I$ have heard of; but Mr. Moor, to be successful, will have to throw his infinitismals to the dogs, and increase his doses. There are other authors of less note, but all follow in one beaten track, from which there seems to be no variableness nor shadow of turning. The minds of Englishmen all follow in one direct line of march, and will do so till the voice of some one will beheard, as did the foolish Galatians.

Prevention.-As has already been stated, it is a contagious disease, and to prevent its spread, isolation, or separation will have to be adopted, to insure immunity to animals not affected.

Epizootic influenza in the horse, pleuro-pneumonia in cattle, took twenty-one years to travel from Europe to the United States. Now whether contagious typhus in cattle will follow so fixed a law as those diseases appear to have done, we may expect to hear of it about 1886 . This disease broke out in England, in June, in 1865, and in one year it destroyed two hundred and fifty thousand, eight hundred and seventy five $(250,875$, head of cattle, and thirty-three thousand, two hundred and thirty-five $(33,235$,$) recovered. All the others died, or were killed to$ prevent contagion, and spread of the disease. To enter into a history of this disease would take up more time 
and space than can be afforded, and would only be a rehearsal of what is recorded in the 1865 visitation to England. For an English history of this disease in all parts of the world, the reader is referred to a work of six to seven hundred pages by Professor Gamgee, published in London. In this work much letter press is to be seen, but not much to be learned from it, and no such views as I have here presented, are even hinted at. Parliamentary reports, Order from Privy Council, letters, editorials and suggestions contained in the London Times, together with the reports and actions of the International Veterinary Congress, held on the Continent of Europe, go to make up this large book.

\section{Udder Diseases.-(See Garget.)}

\section{Urine.-(See Red Water.)}

Vazinal Catarrh.-A disease in old cows a few weeks after calving, and resembles, and is treated the same* as Leucorrhoea. (Which see.)

\section{Varicella Boum.-(See Chicken Pox.)}

\section{Vertigo.-(See Stomach Staggers and Hoven.)}

Warbles.-The product of the œstrus bovus, or ox fly, from having deposited its ova or egg in the skin. It is a disease of the summer months, and is characterized by tumors varying in size from a grain of corn to that of a hickory nut.

Treatment.-Squeeze the tumor and the larva is forced out, which puts an end to the tumors. 
WOMB.

Water in the Chest.-(See Pleuro-Pneumonia.) Whites-(See Leucorrhœea.)

Worms.-(See Hoose.)

Womb.-(See Falling of the Womb.) 

PRIZE ESSAY

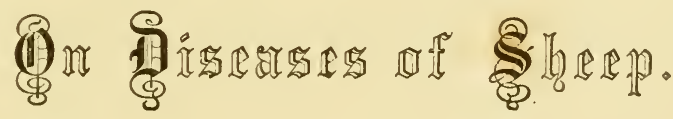





\section{ESSAY ON DISEASES OF SHEEP.}

The following was the Essay for which the United States Agricultural Society awarded their medal and diploma, in the year 1861, to Robert McClure, of Philadelphia, $\mathrm{Pa}$.

The diseases of sheep which are laid under contribution are:-First. Those that are the most frequent and destructive, therefore of the greatest interest and concern to the sheep farmer. Second. Those to which treatment and preventative means can be employed with profit and success. Third. Those that their pathology have been in a measure or entirely misunderstood, therefore wrongly treated, and preventative measures neglected or misapplied. Fourth. These diseases will embrace a large number of maladies that have been treated of separately in the work by the late Wm. Youatt as independent affections, when literally a great many dis- eases alluded to by this indefatigible writer are but different degrees and conditions of the same morbid phenomena.

Braxy-Enterites.-(Inflammation of the Bowels.) Inflammation of the bowels is commonly known by the 
unmeaning name of "Braxy."* It is a disease of more frequent occurrence among this class of useful animals than any other domestic animal I know of; chiefly affecting young sheep in their first year, in cold and stormy weather, in exposed situations. It is not unfrequently when sheep are feeding on turnips in the winter season, to find in the morning after a cold, wet night, as many as a dozen out of a few score dead, dying, or affected by this disease.

The symptoms are: the sheep will be observed to be very restless; lying and rising; resting on one side and then on the other; walking up and down as if looking for a place to lay down, but can find no place to suit.

Treatment.-Give castor oil, two ounces; calomel, five grains; laudanum, two drachms; molasses, two ounces; beat up with an egg, and as as much warm water as will be sufficient for a small drench, to be poured out of the stroupe or pipe of a common tea or coffee pot; then repeat this in half doses every six hours.

Prevention.-In stormy weather, and when feeding turnips where they are generally fenced in a certain space, remove them to pasture a little elevated, and as sheltered as possible from the storm until the weather improves, and the turnip or other fields become drier; place common or rock salt in covered troughs, which will tend to keep their bowels open; as salt is a good laxative for cattle and sheep.

* As sheep affected by this disease are generally on the turnip field, and in good condition; if so, and the animal very sick never attempt to cure; as in most cases death will take placc before medical agents (be they ever so well directed) can take effect. Therefore it will be the better plan to cut the neck, letting out the blood, thereby saving time and trouble, and preserving the mutton in good order. 
Diarrhœa. - Treatment. - Powdered opium, two grains; powdered gentian and powdered ginger, one drachm each: mix. To be given in an infusion of linseed, and repeated if necessary.

\section{Hydrocephalus Hydatideus, Sturdy, etc.-I am} pleased to think that some addition has been made of late to the stock of veterinary knowledge as regards the useful animals, and I trust that the time is proximate when the good sense of the American farmers will demand that a knowledge of the diseases and general management of sheep shall form a prominent feature in the education of the veterinary surgeon.

Sturdy is a singular disease, a very prevalent and fatal one, if left to run its course; so much so, indeed, that in France alone, no less than one miliion sheep die yearly, or are destroyed by this pest of the ovine race. The symptoms by which this disease is accompanied are as follows: In the commencement, the animals will be observed to stop in the midst of their grazing, and then start away in a gallop over the field. They seem at times to be utterly unconscious where they are, separating themselves from the rest of the herd. By-and-by they become dull, and have a peculiar staggering gait. If there is a brook or rivulet within their reach, you are almost sure to find them standing by it, apparently becoming giddy, not unfrequently tumbling in, and are thus lost. They lose flesh, the countenance becomes haggard, and subsequently, the animals thus affected commence a rotary motion, going round and round in the same directions, with the head inclined to the same side of the body. Now it almost ceases to feed or ruminate, as it cannot restrain the rotary mo- 
tions, and is becoming rapidly blind. Death generally ensues from starvation.

Veterinary writers and agriculturists have differed very materially as to the cause of hydatid, some attributing it to certain poisonous plants; but these have never been pointed out. Others consider it a species of serous apoplexy, and others contend that it arises from local weakness of the brain, etc., etc. Autopsical examinations have, however, proved it to arise from a different cause, viz: Coenurias cerebralis, or hydatid in the brain, found floating in a serious fluid, contained within a sac or bladder, thus constituting Sturdy, Gid, Turnsick, etc. It attacks sheep from the sixth to the eighteenth month.

* "Certainly" the most common cause consists in the lamb or young sheep picking from the pastures the ova or larvæ of the tonia solium, which infests the shepherd's dog. If Echinococcus, polymorphus or vetrinorium, be swallowed by the dog, they are developed into that of tapeworm, with but fer serrations. The minute ova are gathered and swallowed with the food of the sheep or lamb, and are taken up from the surface of the intestines. They find their way into the blood, and finding a convenient nidus among the loose textures of the brain, are there deposited. Nature sets to work and encloses these foreign bodies in a membranous sac, so that otherwise they may not produce fatal consequences, and in the short period of three months they are found to have reached the size of a filbert.

The larvæ of the distomum hepaticum found inhabiting

*Findlay Dun. 
the biliary ducts of the ox, sheep, and goat, if swallowed by young sheep, generate the hydatids or Sturdy.

Conversely, if these hytatids are swallowed by the dog, they are developed into tape-worms. Hydatids may be prevented in sheep by curing or preventing tape-worms in other animals, especially the dog. As before mentioned, hydatids only affect young sheep, and spring from the ova of the trenia. Herds managed without dogs are found to be quite free from the disease; however, dogs may be used with impunity when the sheep are upwards of eighteen months old.

Treatment.-(The following is from the Farmer and Gardener, by the present writer.)-The treatment of this infection, until lately, has been varied, barbarous, and cruel; one practising and recommending the cutting and pulling out the ears, and another of hunting the poor animals with dogs, and, if possible, running them over some precipice, frequently maiming or killing them. A strong knitting-needle forced up the nostrils has long been used, with occasional success; but a small case of instruments, consisting of four pieces, is much better and safer, and, if properly used, will cure from seventy to eighty shcep out of a hundred thus affected, and can be used by any intelligent shepherd or farmer with perfect safety. In operating, observe carefully the side to which the sheep turns, as it invariably turns to the side of the skull which is affected, and which must be first operated upon. Sccure and tie fast all the four feet, and place the animal on a table. Let your assistant sit down on the end of the table. Clip away all wool from the brow, sides, and crown of the head. Ascertain by the fingers, if the skull yields on pressure at any particular spot on the side to which the sheep turned: if so, shave the wool from the soft part. 
Use first the instrument with the adjusting screw, its use being to prepare for the other, it being made a little wider than the trocar, with canula, so that the silver may not be broken in piercing the bone. Care, however, must be taken to stop when the point is fairly through the skull: this is easily ascertained. The guard must then be screwed back. This done, take it out and insert the trocar and canula in the same hole, until past the split in the silver canula; then withdraw the trocar, leaving the canula sticking in the head, when the water will be forced from the hydatid, showing that it has been pierced. Put the point of the syringe into the canula, and draw out repeatedly, emptying each time until no more water will come out, or the cyst, has been drawn into the canula, which now withdraw, taking care that the skin of the hydatid be caught hold of by the forceps, which the operator should have ready in his hand. Having removed the hydatid, leave the mouth of the wound open, only placing a piece of clean rag as a pledget over it, as water will escape for a day or two. Put on the angular hood to keep the cloth and padding in their place, and secure it by worsted ties. Care must be taken, if the sheep has not been able to eat for some days, that it get a bottle of gruel before operating, as the less disturbance it is subjected to for some hours after the operation the better, as the brain takes a little time to adjust itself to the cavity that has been made by the removal of the hydatid. Neither must the sheep be put on too rich pasture, as on recovering they are apt to take too much food, which may cause inflammation in the head. Mark well the side that has been operated on, as it very frequently happens that there are more hydatids than one. In a week or two, if the sheep be observed turning to the opposite side, secure 
it a second time, and proceed as before. There are occasionally more than one or two in the same subject and at the same time; indeed I have known as many as five, and not one of them less in size than the yolk of an egg; but two are quite common, one on each side of the brain, nearly under the seat of the horn. Occasionally there is one behind the first lobe of the brain, which is also easily taken out.

Louping Ill.-(Tabes Dorsalis.)-(Phrenitis.) - In every day language this is properly called inflammation of the brain. Youat, in his work on sheep, has been at the trouble to write out no less than six different diseases, all of which might have been treated under this head, which are essentially the same in character, cause, and effect. It is characterized by extreme dulness, followed by excitement and madness. In horses, these symptoms continue for about two days; but in cattle and sheep, much longer. The seat of the disease is the pia matter, or the vascular internal membrane of the brain, which is found after death rough and yellow, with extravased lymph and serum at the base of the brain.

Treatment.-Give Croton oil, 6 drops; Sirup of ginger, 1 tablespoonful; in a little gruel, so thin as it will run from the teapot. Apply cold water or ice to the head, and see that the poor animal does not hurt itself against the wall or fence.

The causes of this disease are the result of injury to the head; but it also arises from other causes besides external injury-the principal, exposure to cold, or a powerful sun in an exposed situation. But the most common causes are the eating from the pasture poisoning plant, or weeds, as the Atropa belladonna, (deadly nightshade,) Conium maculatum, (common hemlock,) 
Cicuta virosa, (water hemlock, Delphinium staphysagria, (stavesacre,) Felis foomina, (female fern;) all of which, if taken in sufficient quantity, will produce disease in the brain. I could easily swell the list of poisonous plants, but it is not my intention to enter into that which is unnecessary.

Paronychia Ovium.-(Foot Root.)-This disease has been more prevalent these last thirty years than heretofore, which is accountable for in the varieties and breeds of sheep and in the increasing number; so much so, that farmers cannot find highland pasture sufficient for them, so they are brought down and put on moist lowland pasture, where the sheep can find plenty of food without travelling much; hence, the growth of horn is more than the wear, or the supply more than the demand; subsequently the horn becomes over-grown and turns upon the sole, and bruising the sensitive structures, earth and sand soon find their way inta the cavities produced, causing inflammation of the laminæ, lameness, and secretion of fetid pus.

Causes.-As before stated, it results from an increased supply of horn which turns up from the toe, pressing upon and irritating the sensitive structures of the foot.

This affection of the feet is almost certain to follow the removal of heavy bred sheep, such as the black-faced Cheviot and Southdown, from their native and upland pastures to rich meadow and arable lands. Their native walks being rough and rugged-the grass not so plenty, which insure the sheep walking over a large tract of land in order to procure their food-hence the waste and supply of hoof is balanced.

Treatment.-Whenever the sheep is observed to go lame it must be caught and the feet examined. Remove 
all filth and sand; then cut off every loose and unsound portion of horn with a stout pocket knife, and apply the following to the sore parts: Oil of turpentine, two ounces; sulphuric acid, half an ounce; olive oil, one ounce. Mix. To be applied with a small piece of sponge or cloth on the end of a stick; or a goose feather will answer very well. If sores occur on the digital space (divisions) or elsewhere, they should be touched with nitric acid or a little of the old butter of antimony, (tercholide of antimony,) by means of a feather. These appliances should be continued twice weekly, or as often as is necessary, until amendment takes place.

Prevention.-To prevent this disease, the growth of horn must be kept down; and if the sheep are on soft land, they must be examined every month, and superfluous horn must be cut off. Some sheph^rds advocate driving along a hard road to wear the hoofs; I do not recommend such a course, as it does sheep a great deal of harm. The better way is to drive them into a sheepfold and examine their feet.

Draining and liming the land very often puts a stop to foot-rot; the drainage to harden and dry the land, the liming to remove the soft mossy herbage which is a great cause of foot-rot in sheep. Foot-rot in sheep is not contagious; but foot-rot in cattle, occuring in connexion with murrian or vesicular epizootic, is deadly contagious.

Pneumonia. - Treatment. - Nitre, one drachm; digitalis, twenty grains; tartar emetic, twenty grains: mix. To be given in linseed tea, once per day in inflammation of the lungs, common cold accompanied with fever, or any disease, that is necessary to arrest the inordinate action of the heart. 
Psora, Scabies.-(Scab.)-When a sheep or flock of sheep are affected with scab, which is easily known by their contirual standing by the fence and rubbing posts, scratching, and tearing the wool off their back and sides, they will scarcely ever be seen grazing or lying down quietly and remasticating their food, as is the case with sheep in perfect health. This disease in sheep is the same as mange, in horses, dogs and cattle; and which result from the attacks of minute insects; or, mites (acari) burrowing in the skin, producing great irritation and itching, and the eruption of small pimples, with dryness, scurfiness and baldness of the skin. This disease is contagious; therefore, to prevent the spread of the infection, the healthy must be separated from the diseased. Keeping sheep clean, and occasionally washing in the summer season, in a great measure prevents a suitable nidus for the acari, and diminishes the prevalence of scab. Before putting healthy sheep on the same pasture, it will be necessary to wash all the rubbing posts with the solution of the chloride, or common lime, which will literally burn up the virus. To make the impossibility of infection doubly sure (neat cattle and cows are as liable to take it as sheep) would be to take a crop of hay or turn it over for a crop of corn, or any other purpose.

Treatment.-The object to be attained in curing this disease is the destruction of the acari, which is necessarily the same in all animals, but in sheep the material used must be in a fluid form, so as to penetrate the wool.

The following is both an excellent cure and preventive of scab in sheep:-R. Acid Arseniosi, lbij; Sulph. Ferri, lb.cev; Aquæ, cong. lxv. Translation.-Powdered arsenious acid, two pounds; sulphate of iron, two hundred and five pounds; water, sixty-five gallons: mix. 
Boil until the fluid is reduced to one-third, and then add as much water as has been lost by evaporation. This preparation is the celebrated "Bain de Tessier," so highly prized in France. The following will answer the same purpose, which will not stain the wool, as it contains no sulphate of iron, and will, no doubt, be preferred by many to the preceding one on that account. Arsenious Acid, 2 pounds; Sulphate of Zinc, 10 pounds; Water, 60 gallons: mix. Prepare as the foregoing one. Each of these valuable sheep-dipping mixtures are sufficient to cure and dip one hundred-sheep.

The way sheep-dipping mixtures are sometimes used is far.wrong, as was the case of the celebrated sheep-poisoning case at Burton, England, where the animals were driven, immediately after their bath, containing twenty ounces of arsenic, twenty ounces of soda-ash, two ounces of sulphur, to their pasture, with their wool saturated with it, and consequently dripping from the wool on the grass that the sheep were just eating.

The improved plan in dipping sheep are, First, The solution or the mixture is to be put in a tub, or other vessel, sufficiently large to allow the sheep (except the head) to be immersed in it, without the mixture running over the sides of the tub or vessel. Second. When the sheep is taken out, it must be placed in another tub or vessel, and the liquid pressed from the wool, and returned again into the dipping vessel. Third. The sheep must be kept enclosed in a sheep-fold, or other sufficiently airy place, where there is no food of any kind that a sheep will eat, until the wool is perfectly dry.

Several of the veterinary professors of the Edinburgh veterinary colleges, after this sheep-poisoning case oc- 
curred, instituted a series of experiments to prove whether arsenical baths were dangerous, and if sheep could be poisoned by immersion therein. The following is the result of their experience. (Edinburgh Veterinary Review for April, 1859.)

First. That the immersion of sheep whether sound, or siffering from scab, with extensive eruptions or "footrot," with free exposure of the vascular structure of the feet, in arsenical solutions, varying in strength, is unattended by prejudicial effects, and proves salutary to the diseased animals.

Secondly. That the congregating of sheep after dipping, where food is not allowed them, is unattended with danger; and I can distinctly testify to the absence of all risk from sheep licking each other.

Thirdly. That all sheep having remained damp from twenty-four to thirty-six hours after being dipped, cutaneous absorption of arsenical solution might have been going on rapidly during the prolonged period that the sheep continued wet, but no unfavorable symptoms ensued,-indeed the reverse.

Fourthly. The absence of the slightest change in health, or of the manifestation of symptoms which usually ensue on the administration of arsenic to the lower animals, even in medicinal doses by the mouth, leads us to infer that if a minute quantity of arsenic, in solution, can penetrate the system of a sheep by absorption by the skin, it is quite insufficient to develop the physiological action of the compound.

Fifthly. A solution of arsenic is not more readily absorbed from limited abraded surfaces than from the healthy skin, owing probably to the arsenic acting as an astringent on the sores, and rendering the latter as im- 
penetrable, probably, as the uninjured skin. This remark does not apply to large wounds.

Sixthly. It appears impossible to poison sheep by bathing them in arsenical solutions, provided the liquor is not introduced directly or indirectly into the stomach by the mouth. By the direct means of introduction of the constituents of a "dripping mixture" in the stomach of a sheep, is meant making the sheep swallow the liquid whilst being bathed; and by the indirect means is implied the penetration of arsenical solution into the stomach by food poisoned by the liquid dropping from the bodies of the sheep, the wool of which has not been pressed, or from a number of sheep injudiciously congregated over food they subsequently eat.

Seventhly. Arsenical solutions, whether weaker than ordinary dipping mixtures, or much stronger, are likewise incapable of being absorbed by the skin of sheep or man.

Eighthly. Sheep will partake of grass and other food which has been smeared or soaked with an arsenical solution, notwithstanding the presence of soft soap and soda.

Arsenical baths and mixtures are not only used for the cure and prevention of scab, but are the most highly, and, I think, justly valued preservatives of the fleece of sheep, and is a protection to the propagation of many parasites.

Rot-Cachexia Aquosa.-(Chlorosis.)-The very name of this disease is a terror to many sheep farmers who have suffered and lost so much by it. It is so destructive amongst sheep that it is estimated that in the rainy winter of 1829-30 two million sheep died or were destroyed by this disease alone. It again made its ap- 
pearance in Great Britain the very wet years 1852-53. The symptoms by which this disease can be detected in its early stage are very latent; but if there are suspicions of this disease it can readily be detected by the color of the mucous membranes; as the fauces of the mouth; the appendages of the eye being of a pale yellow; the animal costive and the urine of a high color, thus indicating disease of the liver. As the disease is more advanced there is little difficulty in coming to a true and proper diagnosis of the case, as all the muscular fibre assumes a uniform yellow brown color, and the wool has a matted and dry appearance, and subsequently the muscles waste and digestion is impaired, and at length the animal dies in from two to four months. Where a flock of sheep do not thrive and acquire fat and flesh on good feed and pasture, why, something must be wrong, and the sooner it is found out the better, as they may have acquired the disease before they were bought. The better way would be to have one of the worst ones brought in and destroyed, and then have a thorough examination, which will set every thing to rest, and point out the line of duty which is open to be pursued in the treatment and prevention of the disease ere it be too late to then do good.

It is very frequent in wet seasons, and in wet and undrained lands. It is characterized by an enlarged and softened condition of the liver, and accumulation of the common fluke (distomum hepaticum, in the biliary ducts and gall bladder.

Post-mortem appearances show most clearly that the nature and extent of the disease, as before mentioned. will be found a large number of these flukes in the liver and that organ very much enlarged, soft, and flabby; 
ROT.

the bile and all the serous fluids of a yellow color, and, on account of the debilitating nature of the disease, a large quantity of the exudative matter is found in the belly and chest. There is one appearance that will never deceive: the liver is not of that pale and I would say cooked appearance which is usually presented after ordinary "Hepatitis," but in most cases all covered with livid spots not unlike the mottled appearance of the lungs of cattle that have died of epizootic pleuro-pneumonia. In a word, the liver is the only true seat of this disease.

Cause.-The cause of this disease, until lately, was often a surmise and supposition. Then the question arose why it has been a precept with careful shepherds not to let the sheep graze on dewy grass, or wet and marshy land? Why the dew and wet land was considered so dangerous none could tell, and least of all the bucolic guardian. Although unable to give a reason why, he could aver and prove that it was so. The rational is, that many creatures pass the early portion of their predatory existence in the bodies of one species of animal, and their maturity in another. Their eggs are deposited in the former, but are not developed until expelled and cast upon the earth or manure-heap to shift for themselves.

They lie on the smoking dung-pile or such places, and far away from the sheep, in which they can find food and develop themselves. The rain washes them into the earth, or they are carried to the fields with the manure. The humidity serves to develop them: they fix themselves upon the moist grass, and with it these tiny enotozoa are carried into the stomachs of the sheep. Once there, their work is soon accomplished.

Treatment.-The successful treatment of this disease 
embraces that of prevention also, as before mentioned: it is chiefly caused by wet and damp pasture landshence the proposition for their restoration will be, First, their removal to a high and healthy pasture, and give them hay and nourishing food, such as a little bruised oil cake; or linseed-meal, and place covered troughs in the field containing rock, or common salt, of which the sheep is very fond of licking, and moreover regulates their bowels, and gives them an appetite for food and water. Second, give each sheep epsom salts, two ounces; oil of turpentine, one ounce; pulverized ginger, half an ounce. To be given in half a pint of molasseswater, to be followed up with tonics, if they are found necessary. The following will answer every good purpose: Sulphate of iron, in powder, ten grains; gentian, in powder, thirty grains; ginger, in powder, thirty grains. To be given in linseed-meal gruel once a day for a few days. It is the experience of almost every sheep-farmer, and sometimes too dearly bought, that to place sheep on wet and marshy land, even for a few days, is certain to generate this disease, notwithstanding the animals may have been in perfect health prior to his placing them there. Before placing sheep on pasture where there is access to any bog or marsh it will amply repay the time and trouble to fence it in; or have sufficient sheep-drains opened through it six months before any sheep should be put on it.

Sheep-drains are wide, and not filled up with tiles or stones, but left open. The shepherd can find time to keep both the sheep in order, and the drains clean of rubbish, except in the lambing and clipping season.

Small-Pox.-Variola Ovina.-Fortunately this disease has as yet been confined to Europe, and more espe- 
cially the continental portion, where it may be said to be never absent. In France and Italy it occurs frequently, and causes much loss and destruction. England was visited with it in 1847, when it was communicated to a flock at Datchett, and another at Pinnar, by some Merinoes from Spain. In 1862 it again occurred, but this time very suddenly, and in a very severe form among the flocks of Wiltshire; for which re-appearance neither infection nor traceable contagion could be assigned as the cause. The light shed upon it would appear to be an instance of origination anew of a more malignant type of variola ovina. Such is, in fact, assigned to this disease in Africa, it being well established that certain injurious atmospheric influences produce skin diseases and facilitate the appearance of pustular eruptions. From the nature of the disease in its being produced both by atmospheric causes and contagion, it soon assumes an epizootic form, and causes more destruction than any other malady affecting this useful animal. Out of a flock of 1720 , ninety-two were attacked in a natural way, of which fifty per cent. died. Of eight hundred inoculated cases, only thirty-six per cent. died. The mortality from this disease is never less than twenty-five per cent. and not unfrequently whole flocks have been swept away, death taking place in the early stages of the eruption, and others in suppurative and ulcerative stages.

Symptoms.-They may be mapped out as follows: The animal is seized with a shivering fit, succeeded by a dull stupidity, which remains till death or recovery takes place. On the second or third day pimples are seen on the inside of the thighs and arm-pits, accompanied with redness of the eyes, complete loss of appetite and other 
symptoms which are common to other diseases of the same animal.

Prevention.-Two plans are resorted to for the purpose of preventing the spread of the affection, which promise a certainty of success. The first and best plan is, isolation and destruction. This plan proved a great protection to the sheep farmers of Wiltshire in 1862. In well known epizootic diseases, and when individual cases occur which when pointed out and well recognized, as soon as the fever sets in, and before the eruptions appear, they should be slaughtered at once and buried. The loss of one or two sheep is nothing to the consequences of the spread of a disease of this kind. By doing so the disease has been known to be confined to a few cases in a large flock.

Treatment.-In treating this disease, resort has been had to a plant called sarracenia purpura, Indian cup, or pitcher plant, and used for treating this disease in man by the Micmac tribe of Indians in British North America. (See Medicines in Part II. of the book.) Take from one to two ounces of the root, (dried if to be had) and slice it in thin pieces; place in an earthen pot; add a quart of cold water, and allow the liquid to simmer gently over a slow, steady fire from two to three hours, so as to lose one-fourth of the quantity. Give of this tea or decoction three wine-glassfuls at once, and the same quantity from four to six hours after, when a cure will generally be effccted. Weaker and smaller doses are certain preventives of small-pox, whether in men or animals. The public are indebted to Dr. Norris, Physician to the Halifax (Nova Scotia) Dispensary, for the manner of preparing this highly important article. Sulphurous acid gas will be found useful in small-pox. For manner of using, see PART II. of the book. 


\section{ENGRA VINGS.}

\section{PART I.}

PAQE

Abscess Lancet....................................... 18

Artery Forceps...................................... 23

Arterial Circulation.......................Frontispiece

Bullet Forceps...................................... 99

Ecraseur..................................... 35, 113

Florence Flask.................................. 52

Foot Lancet.......................................... 77

Injection Syringe.................................. 112

Liston's Bone Forceps............................... 134

Needle and Wire....................................... 163

Operating on the Eye .............................. 62

Operating Knife.................................... 192

Quittor Syringe.................................... 153

Spring Lancet...................................... 114

Tooth Rasp. ......................................... 132

Tooth Forceps.......................................... 189

Tracheotomy Tube ................................... 190

Trephine............................................. 191

Trocar, straight.................................... 114

Trocar, curved...........................................114 


\section{PART II.}

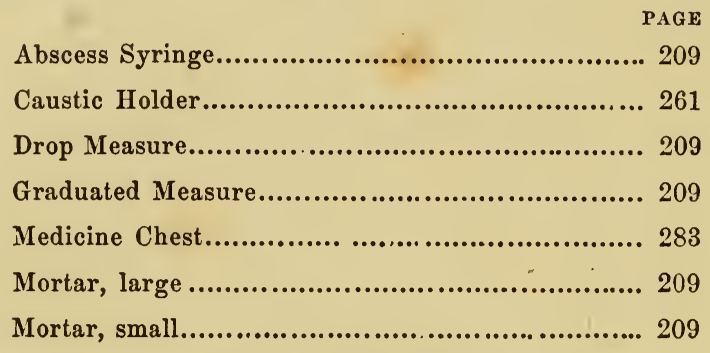

PART III.

Ox Head............................................... 290 


\section{IN D EX.}

\section{PART I.}

A.

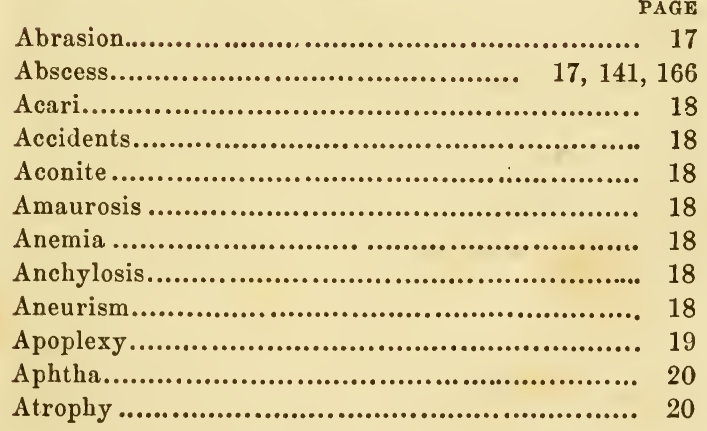

B.

Back Sinews. ............................................. 21

Baldness............................................ 21, 171

Belly Ache.................................................. 21

Big Head....................................................... 21

Bishoping .............................................. 21

Bite of Mad Dog............................................. 21

Black Tongue................................................ 132

Bladder Diseases........................................ 22 
Bladder, Inflammation of ............................. 22

Bladder, Calculi in...................................... 22

Bleeding.................................................... 23

Bleeding from Wounds.................................. 23

Bleeding from the Air Passages........................ 24

Bleeding from the Skin................................ 24

Bloody Urine............................................... 25

Blood ................................................... 25

Boils.................................................... 26

Bots ................................................... 26

Bowels, Diseases of............................................ 26

Bowels, Inflammation of ............................. 26

Brain Diseases .......................................... 26

Breaking Down........................................ 27

Breathing.............................................. 13

Breathing Short..................................... 27

Breeze Flies............................................. 27

Brittle Feet................................................ 27

Bronchi ................................................ 27

Bronchitis ................................................... 27

Bronchitis, Acute.................................... 28

Bronchitis, Chronic.................................... 29

Bronchocele ............................................... 30

Broken Knees......................................... 30

Bruises of the Sole..................................... 31

Burns and Scalds....................................... 31

Bursa Mucosa, enlarged............................. 32

\section{c.}

Calculi.................................................... 32

Cancers.................................................. 32

Canine Rabies.. ......................................... 33

Capped Elbow .......................................... 33

Capped Hock............................................ 34

Carditis ...................................................... 34

Caries.................................................... 34

Castration............................................... 35

Cataract ................................................. 36

Catarrh................................................. $\quad 36$

Causes of Disease........................................ 7

Cautery..................................................... 36

Chest Diseases ........................................... 36 


\section{INDEX.}

Chest Founder.

Chill

(n...................... 36

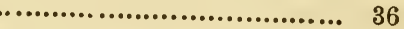

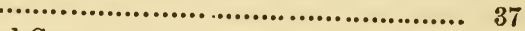

Clover, Second Crop........ i................................. 163

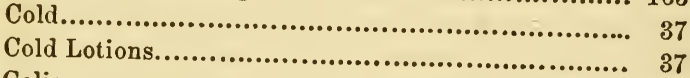

Colic..................................................... 39

Colic, Spasmodic.......................................... 39

Colic, Stercoral......................................... 39

Colic, Flatulent .......................................... 40

Coma ........................................................ 41

Congestion ............................................. 9, 42

Constipation.............................................. 42

Consumption ............................................ 42

Contagion ............................................. 43

Contraction............................................. 43

Convalescence............................................... 43

Corns ....................................................... 43

Coryza Gangrenosa ........................................ 43

Corrosive Sublimate..................................... 44

Cough..................................................... 44

Cough, Chronic.......................................... 44

Counter Irritants....................................... 44

Cow Hock............................................... 44

Cramp...................................................... 44

Cramp of Hind Leg.......................................... 4

Crib Biting............................................... 45

Curb .................................................... 46

Curby Hocked............................................ 46

Cutaneous Diseases....................................... 46

Cutting................................................ 46

D.

Death

Deafness............................................... 54

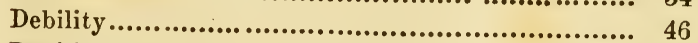

Debility, Simple........................................ 46

Debility, General ...................................... 47

Dedication ................................................ ${ }_{3}$

Deformities................................................ 47

Depression........................................... 10 
Diabetes................................................. 48

Diaphoretics..... ........................................ 48

Diarrhœa ........................................... 14, 49

Diarrhœa, Simple..................................... 49

Diarrhœa, Continued.................................. 49

Diathesis................................................. 49

Diet......................................................... 50

Disinfectants.......................................... 50

Disinfectants, Natural................................ 50

Disinfectants, Artificial............................... 51

Distemper........................................... 53

Diuretics.................................................. 53

Dropsies................................................. 53

Dropsy in the Chest.. ................................. 53

Dropsy in the Belly.................................... 53

Dropsy in the Heart.................................... 53

Dropsy in the Legs................................... 53

Dysentery........................................... 54

E.

Ear Diseases.......................................... 54

Ear, Small Tumors in the............................. 54

Ear, Injuries of the...................................... 54

Ear, Foreign Bodies in the............................ 54

Ear, Abscess in the................................. 55

Ear, Dry Gangrene of the........................... 55

Ecchy mosis......................................... 55, 128

Eczema............................................ 55, 173

Elephantiasis......................................... 55

Embrocations .............................................. 55

Emetics............................................... 55

Emphysema .......................................... 55

Encysted Tumors.................................... 56

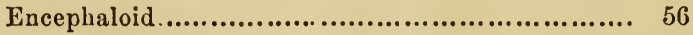

Enema................................................... 56

Enteritis.............................................. 56

Enzootics............................................. 56

Ephemeral............................................. 56

Epidemic............................................. 56

Epizootic................................................. 56

Epilepsy............................................. 56

Epiphora............................................ 56 
Epsom Salts........................................... 56

Eruptions.................................................. 56

Erysipelas ............................................ 56

Exostosis................................................ 57

Eye Diseases......................................... 57

Eye, Amaurosis of the................................. 58

Eye, Floatıng Spots in the........................... 59

Eye, Inflammation of the............................ हy

Eye, Polypi in the................................... 60

Eye, Spots on the..................................... 60

Eye, Weeping from the............................... 61

Eye, Closing of the.................................... 61

Eye, Hair Growing in the............................. 61

Eye, Swelling of the Eye-lids......................... 61

Eye, Ophthalmia of the................................ 61

Eye, Operation on the................................. 62

Eye, Moon Blindness of the.......................... 63

Excretions............................................ 105

F.

False Quarter.................................. 64, 76

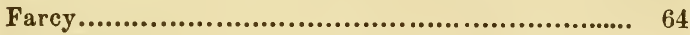

Fatty Tumor ....................................... 68

Farrier.............................................. 68

Favus.................................................... 174

Fever............................................. 11, 68

Fever, Idiopathic.................................... 11

Fever, Sympathetic...................................11, 68

Fever in the Feet..................................... 69

Fibroma.............................................. 69

Firing Horses ......................................... 69

Fistulæ................................................ 69

Fistulæ in the Foot.................................... 71

Fistulæ in the Withers................................ 71

Fits................................................... 72

Flaxseed ............................................... 72

Fomentations......................................... 72

Foot Diseases........................................... 73

Foot, Canker of the................................... 74

Foot, Contraction of the ............................... 74

Foot, Corns of the...................................... 75

Foot, Inflammation of the.............................. 76 
Foot, Pricks in the................................... 76

Foot, Bruises of the ................................... $\quad 77$

Foot, Sand-Cracks of the............................ 77

Foot, Navicular Disease of the....................... 78

Foot, Seedy Toe of the................................. 79

Foot, Pumiced Sole of the................................ 80

Foot, Thrush of the.................................. 80

Foot, Quittor of the.................................... 81

Founder .................................................. 82

Founder, Chronic...................................... 84

Fractures............................................... 84

Fractures, Simple..................................... 84

Fractures, Compound................................... 84

Fractures, Comminuted.................................. 84

Fracture of the Hip................................. 86

G.

Gangrene............................................. 88

Gastritis Mucosa.......................................... 88

Glanders.................................................. 91

Glass Eye............................................ 95

Gleet, Nasal........................................... 95

Granulation.............................................. 96

Gravel in the Foot........................................ 96

Grease........................................................ 96

Grease, Shot of........................................ 97

Gripes................................................... 98

Grogginess............................................... 98

Grunter... ............................................. 99

Gullet, Obstructions in................................. 99

Gun Shot............................................. 99

Gutta Serena............................................. 99

H.

Heart, Disease of..................................... 100

Heaves .......................................................... 101

Heat........................................................ 103

Hepatic Diseases....................................... 103

Herpes.................................................. 103

Hereditary Diseases..................................... 103

Hide Bound............................................ 104 
Hip Joint Diseases...................................... 105

Hock, the............................................... 106

Hocks in the Eyes....................................... 107

Horse, Natural History of............................. 107

Horse Fly............................................ 107

How to Observe Disease................................ 8

How Diseases are Cured without Medicine.......... 14

Hydrothorax................................107, 126, 127

Hydrocele ........................................... 108

Hydronemia............................................. 108

Hydrophobia............................................ 108

Hypertrophy........................................... 109

Hysteria ............................................... 109

I.

Indigestion.......................................... 110

Infection.............................................. 110

Inflammation........................................ 9, 110

Influenza ................................................ 110

Injections............................................... 112

Instruments............................................ 113

Intestines ................................................ 115

Itch.............................................. 115, 172

Interfering.......................................... 115

Introduction ......................................... 7

J.

Jack ..................................................... 116

Jaundice ................................................. 116

Joint Diseases.......................................... 116

Jugular Vein............................................ 116

K.

Kidneys, Diseases of ................................. 117

Knees, Broken......................................... 118

Knee Joint................................................ 118

Knee Joint, Loose Cartilage in........................ 118

Knuckling ............................................. 118 
INDEX.

L.

Lameness .............................................. 14, 119

Lamanitis................................................. 119

Lampas ...................................................... 119

Laryngitis ................................................... 120

Leg, Fractures of........................................ 120

Lice............................................................ 120

Ligaments.................................................... 120

Lipoma................................................... 120

Liver......................................................... 120

Liver, Inflammation of.................................... 121

Lock-Jaw.................................................... 122

Loins, Sprain of........................................ 124

Loss of Appetite............................................. 124

Lumbago ................................................... 124

Lungs, the ................................................ 124

Lungs, Inflammation of the................................. 125

Lungs, Abscess in the...................................... 127

Lungs, Adhesions of the .................................... 127

Lungs, Congestion of the............................... 128

Lungs, Air in the.............................................. 128

Lymph...................................................... 128

M.

Madness ........................................................ 128

Mad Staggers ................................................ 129

Malignant .................................................... 129

Malignant, Epidemic.. .................................. 129

Mallenders ................................................ 129

Mange.............................................. 130, 172

Materia Medica............................................ 130

Megrims...................................................... 130

Melanosis................................................ 32, 130

Melanoid ................................................. 32,130

Mesentery ................................................... 130

Mesenterica................................................ 131

Metastasis ................................................... 131

Moon Blindness....................................... 63, 131

Mortification....................................... 10, 88, 131

Moribund ..................................................... 131

Mouth Diseases.............................................. 131 
Mouth Scald.............................................. 132

Mouth, Wounds of the ................................. 132

Mouth, Thrush in the.................................. 132

Mucus.................................................... 133

Mucous Membrane....................................... 133

Myalgia ................................................ 133

N.

Narcotics ................................................ 133

Nasal Gleet........................................ 95, 134

Navicular Disease................................ 78, 134

Necrosis .......................................... 34, 134

Nephritis .................................... 117, 134

Nervousness ............................................. 135

Neurotomy.............................................. 135

Nose, Diseases of............................. 36, 37, 136

Numbness........................................... 136

\section{O.}

Obesity........................................... 136, 145

Edema ............................................. 53, 136

Estromania............................................. 137

Omentum .................................................. 137

Open Joints....................................... 116, 137

Ophthalmia...................................... 61, 137

Ossification ............................................... 137

Osteology............................................... 137

Osteo-porosis..................................... 34, 137

Osteo-sarcoma..................................... 34, 137

Ostitis ..................................................... 137

Overreach............................................... 137

Ozæna.................................................. 138

P.

Palliatives. ................................................ 138

Palsy...................................................... 138

Paralysis................................................. 138

Parotid Duct, Distended.................................. 138

Parotid Duct, Open and Fistulous..................... 139

Pathology ................................................ 139

Patella, Dislocation of................................ 140 
Pasterns.................................................. 140

Pelvis .................................................. 140

Pelvic, Abscess........................................ 141

Penis, Hanging Out................................... 142

Peristallic................................................. 142

Peritonitis ........................................... 142

Periosteum.............................................. 142

Phagedæna.............................................. 142

Phlebitis........................................ 116, 144

Phlegmasia Dolens ................................ 98, 144

Phrenitis ................................................ 144

Physiology............................................. 144

Physicking.............................................. 144

Pleurisy....................................... 126, 145

Pleurodynia........................................... 145

Plethora......................................... 136, 145

Pneumonia........................................ 125, 145

Pneumothorax ............................................. 128

Poisons..................................................... 145

Poisons, Irritant...................................... 145

Poisons, Narcotic....................................... 146

Poll Evil................................................ 147

Polypi............................................ 60, 148

Porrigo................................................ 174

Predisposing Cause................................. 7, 149

Prick of the Foot............................... 76, 149

Principles of Disease................................... 9

Procidentia............................................... 149

Prophylactics............................................ 149

Proud Flesh............................................. 149

Prurigo................................................ 150

Psora.................................................... 172

Pulse ............................................. 12, 150

Puncture................................................ 150

Purgatives........................................ 144, 150

Purpura ............................................... 150

Pus ............................................. 151, 164

Putrefaction..................................... 43, 151

Pyæmia................................... 7, 91, 152 
Q.

Quack Medicines........................................... 152

Quinsy......................................................... 153

Quittor................................................. 81, 153

R.

Rabies.................................................. 108, 15s

Rachitis.............................................. 47, 153

Resolution............................................... 10, 153

Respiration ..................................... 13, 27, 153

Revulsion........................................... 131, 154

Rheumatism..................................... 82, 124, 154

Rheumatism, Chronic..................................... 156

Rheumatism, Accompanying Diseases of the Throat 156

Ring Bone................................................. 157

Ring Worm........................................ 157, 174

Roaring................................................ 99, 158

Round Bone................................................ 158

Rowels....................................................... 158

Ruptures................................................. 158

Rupture, Inguinal....................................... 158

Rupture, Scrotal...................................... 159

Rupture, Congenital...................................... 160

Rupture, Ventral........................................... 161

Rupture of Castration.................................... 161

S.

Saddle Galls................................................. 163

Sallenders............................................. 129, 163

Saliva....................................................... 163

Salivation...................................................... 163

Sand Cracks..................................................... 164

Sanious Pus......................................... 151, 164

Scabies...................................................... 172

Scald Mouth.............................................. 132, 164

Scarlatina...................................................... 164

Scratches ............................................ 142, 164

Schirris ............................................ 30, 130, 165

Scouring............................................ 14, 49, 165

Scrotum................................................... 108, 165

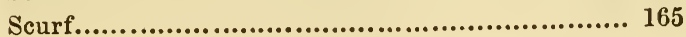


Second Crop of Clover.................................... 163

Secretion............................................... 165

Sedatives................................................ 165

Serous Abscess................................17, 141, 166

Serum ................................................. 165

Seton................................................... 166

Shivers.................................................. 166

Shoeing.................................................... 166

Shoeing Sound Feet.................................. 167

Shoeing Unsound Feet................................ 167

Shoulder Lameness....................................... 168

Shoulder Joint Lameness.............................. 169

Side Bones..................................... 157, 170

Sinus........................................ 69, 71, 170

Sitfasts............................................... 170

Skin Diseases..................................... 21, 171

Slobbering....................................... 163, 175

Slough................................................ 175

Sores.................................................... 175

Sore Foot.........................................73, 175

Sore Mouth...................................137, 175

Sore Throat......................................131, 175

Sore Back............................................. 175

Sore Neck............................................ 175

Sore Shoulders......................................... 175

Soundness............................................... 176

Spavin ............................................... 176

Spavin, Bog.......................................... 177

Spavin, Blood........................................... 177

Spavin, Bone.......................................... 178

Spavin, Occult......................................... 178

Speck on the Eye..................................... 60, 179

Speedy Cut............................................ 179

Spleen Diseases................................. 32, 179

Splint ................................................ 179

Sprains.................................................. 180

Staggers................................................. 181

Staggers, Stomach....................................... 181

Staggers, Grass or Sleepy............................. 182

Staggers, Mad........................................... 182

Staked.................................................. 183 
Stings from Bees, etc................................. 183

Stifle Joint Lameness................................... 184

Stifle Ulceration......................................... 184

Stomach Diseases........................ 39, 88, 181, 185

Stone in the Bladder .............................. 32, 185

Strains ............................................ 180, 185

Strangles....................................... 53, 185

Stranguary..................................... 25, 185

Strangulation ........................................ 186

Stringhalt ..................................................... 186

Suppuration....................................................... 187

Surfeit.......................................................... 174, 188

Swelled Legs................................... 46, 53, 64, 96, 180

Swellings................................................. 188

Sympathy............................................ 11, 188

Synovia................................................. 188

Synchronous .............................................. 189

Systole.................................................... 189

T.

Tabanidæ............................................... 189

Tabes............................................... 131, 189

Talpæ .................................................... 189

Teeth.............................................. 132, 189

Tetanus............................................. 122, 189

Tetter.................................... 21, 171, 189

Thick Wind...................................... 101, 189

Thick Leg........................... 46, 53, 64, 96, 189

Thiselo.............................................69, 189

Thoroughpin.........................................180, 189

Thread Worms............................................ 190

Throat Diseases............................27, 36, 37, 190

Thrush........................................... 80, 190

Thrombus.......................................... 116, 190

Thumps.................................................... 190

Tongue, Lacration of............................. 132, 190

Tonics.................................................... 19 19

Toxicology ................................................ 190

Tracheotomy............................................ 190

Transfusion............................................... 191

Tread................................................ 80, 191 
Treatment of Disease...................................... 13

Trepanning ............................................ 191

Trephine ...................................................... 191

Trismus........................................... 122, 192

Tubercles........................................ 91, 192

Tumors.................................................... 192

Tumor, Encysted.................................. 56, 192

Tumor, Encephaloid .............................. 56, 192

Tumor, Fibrous................................. 69, 193

Tumor, Fatty.................................... 68, 193

Tumor, Cancerous................................ 32, 194

Tumor, Melanoid..................................... 32, 130

Tumor, Bony..................................... 179, 194

Tumor, Varicose ................................ 194, 197

Tympanitis........................................ 40, 194

Typhoid...................................................... 194

U.

Ulcers ....................................................... 194

Ulcers, Healthy...................................... 194

Ulcers, Indolent............................................ 195

Ulcers, Irritable....................................... 195

Urinary, Calculi............................. 32, 185, 196

Urine, Bloody............................... 25, 117

V.

Varicose............................................ 194, 197

Venesection .......................................... 23, 197

Ventilation.......................................... 50, 197

Veterinary Biography................................... 197

Veterinary College ..................................... 201

Vis Medicatrix Naturæ.................................... 14

Vives...................................................... 201

W.

Warts.......................................... 69, 193, 201

Warranty ............................................. 201

Warbles......................................... 21, 171, 201 
Watery Farcy.......................................... 201

Weeping Eye............................................. 201

Wens.................................................... 202

Wheezing ............................................. 99, 202

Whirl-Bone...................................................... 202

Wind-Sucking................................................. 45, 202

W orms.......................................................... 202

Worms, Stomach....................................................... 202

Worms, Fundament............................................ 203

Worms, Strongylus................................................ 204

Worms, Ascarides................................................. 204

Worms, Faliria............................................ 204

Wounds......................................................... 21, 204

Wounds, Contused.......................................... 204

Wounds, Lacerated............................................. 204

Wounds, Punctured............................................ 204

Wourali........................................................... 205

$\mathbf{Y}$

Yellows........................................................... 205

Yellow Water....................................................... 205

Z.

Zoology............................................................. 205

Zumins.................................................................. 205 


\section{PART II.}

A.

PAGE

Acids.................................................... 211

Acid, Acetic............................................. 212

Acid, Pyroligneous .................................... 212

Acid, Muriatic ....................................... 212

Acid, Nitric............................................ 212

Acid, Sulphuric ...................................... 212

Acid, Prussic .. ........................................ 213

Aconite.................................................. 213

Aconite, Tincture..................................... 213

Alcohol ................................................... 214

Aloes.................................................. 214

Alum....................................................... 215

Ammonia.................................................. 215

Ammonia, Carbonate of.............................. 215

Ammonia, Muriate of.................................. 215

Ammonia, Acetate of ................................. 215

Anæsthetics............................................ 216

Aniseed ..................................................... 216

Anodynes............................................... 216

Antimony............................................ 216

Antimony, Tartarized ................................ 216

Antimony, Tersulphuret.............................. 217

Antimony, Terchluride ............................... 217

Antiseptics............................................. 217

Antispasmodics....................................... 217

Areca Nut.............................................. 217

Arnica Montana....... ................................ 217

Arsenic.................................................. 217

Asa fœetida ................................................. 218

Asarabacca.............................................. 218

Astringents............................................ 218

Atropine.............................................. 219 
B.

Belladonna................................................. 219

Benzoin................................................... 219

Benzole.................................................. 219

Bismuth...................................................... 219

Bismuth, Subnitrate of.................................. 219

Bole Armenia ................................................. 219

Borax.................................................. 220

Brimstone.... ........................................... 265

Bromide of Potash....................................... 220

Buckthorn ................................................ 220

C.

Calamine .................................................... 220

Calcium .................................................... 220

Calomel................................................... 221

Camphor.................................................... 221

Cantharides................................................. 221

Capsicum.................................................. 221

Carbolic Acid .............................................. 222

Cardamoms.............................................. 222

Carraway................................................. 222

Carbonate of Lime...................................... 222

Cascarilla.................................................. 222

Castor Oil................................................. 223

Cassia........................................................... 223

Catechu ................................................. 223

Chalk.......................................................... 224

Chamomile.................................................. 224

Charcoal .................................................. 224

Chenopodium, Anthelminticum ......................... 224

Chlorine Gas.............................................. 224

Chloric Ether ............................................ 225

Chloride of Potash...................................... 225

Chloride of Lime ..................................... 225

Chloroform.............................................. 225

Chologogues................................................ 226

Cinchona ............................................... 227

Clysters ............................................. 275 
Cochineal.............................................. 227

Cod Liver Oil.............................................. 227

Colchicum .................................................. 227

Collodion ..................................................... 228

Copper..................................................... 228

Copper, Sulphate of..................................... 228

Copper, Acetate of....................................... 229

Coriander................................................... 229

Creosote ...................................................... 229

Croton Oil ................................................. 230

D.

Digitalis................................................ 230

Disinfectants............................................. 230

Diuretics .................................................. 231

Drenches .................................................. 275

E.

Elecampane........................................... 232

Elm Bark............................................... 232

Emetics....................................................... 232

Epsom Salts............................................ 232

Equivalents.............................................. 287

Ergot.................................................... 233

Essentials Oils............................................ 234

Ether ........................................................ 234

Euphorbium.................................................... 234

Expectorants ............................................ 234

Eye Washes.................................................. 276

F.

Febrifuges............................................. 234

Fern........................................................... 234

Fœnugreek ............................................ 234

Freezing Mixtures...................................... 277

Friar's Balsam............................................ 235

G.

Galbanum................................................. 235

Galls..................................................... 235 
Gallic Acid.............................................. 235

Gamboge............................................... 235

Gentian Root.......................................... 236

Ginger Root.............................................. 236

Glauber's Salts ........................................... 237

Glycerine............................................. 237

Guaiacum ........................................................ 237

Gum Arabic......................................... 237

Gum Senegal............................................ 238

Gum Tragacanth ........................................... 238

E.

Hartshorn .................................................. 238

Hellebore.................................................... 238

Hemlock................................................ 238

Hemp, Indian......................................... 238

Henbane................................................... 239

Hop................................................... 248

I.

Iodine.................................................. 239

Iodide of Potash....................................... 239

Iodide of Arsenic......................................... 240

Iodide of Copper........................................ 240

Iodide of Iron............................................ 240

Iodide of Sulphur....................................... 240

Iodide of Lead......................................... 241

Iodide of Mercury ....................................... 241

Ipecacuanha............................................. 242

Iron........................................................... 242

Iron, Carbonate of...................................... 243

Iron, Oxide of ............................................ 243

Iron, Sulphate of ...................................... 243

Iron, Perchloride of................................... 244

J.

Jalap................................................... 245

Juniper Berries........................................ 245

K.

Kino...................................................... 245

Kousso.................................................. 245 
I.

Laudanum .............................................. 245-255

Laxative...................................................... 245

Lead....................................................... 245

Lead, Oxide of............................................... 246

Lead. Iodide of ............................................... 246

Lead, Sugar of............................................. 246

Lime...................................................... 246

Liniments..................................................... 277

Linseed ...................................................... 246

Linseed Oil...................................................... 246

Liquorice Root............................................. 247

Lobelia.................................................... 247

Logwood ...................................................... 247

Lunar Caustic................................................. 248

Lupuline...................................................... 248

Lytta Cantharides.......................................221-248

$\mathbf{M}$.

Magnesia..................................................... 248

Magnesia, Carbonate of..................................... 248

Magnesia, Sulphate of....................................... 248

Manganese........................................................ 248

Marsh Mallow.................................................. 248

Marigold..................................................... 249

Medicines to be kept....................................... 283

Mercury ........................................................ 249

Mercury with Chalk.......................................... 249

Mercury, Bi-Chloride of.................................. 249

Mercury, Nitrate of ........................................... 250

Mercury. Oxide of.......................................... 250

Mercury, Sulphate of...................................... 250

Mereury, Chloride of..................................... 250

Mercury, Ointment of ..................................... 250

Mezereon .................................................... 250

Morphia .............................................250, 254

Muriatic Acid.............................................. 212

Mustard Seed............................................... 251

Myrrh .................................................... 251 


\section{N.}

Nareotics.................................................... 251

Neatsfoot Oil................................................ 251

Nervines..................................................... 251

Nitric Acid.................................................. 212

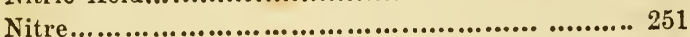

Nux Vomica ................................................... 252

Nux Vomica, Alkaloid of................................ 252

o.

Oak Bark...................................................... 253

Oils............................................................ 253

Ointments.................................................... 279

Ointments, Fixed........................................... 254

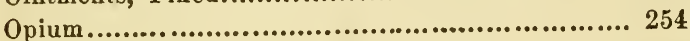

Opium, Tincture of........................................ 255

Opium, Alkaloid of.......................................... 255

Opium, Wine of ......................................... 255

Opodeldoc ................................................. 261

\section{P.}

Pareira Brava.................................................. 255

Peach Leaves.................................................. 255

Peppermint ........................................................ 256

Pepper...................................................... 256

Pepper, Black............................................. 256

Pepper, Cayenne.......................................... 256

Pepper, Jamaica............................................ 2256

Pepsin.......................................................... 256

Petroleum .................................................... 256

Phosphorus ..................................................... 257

Phosphoric Acid........................................... 257

Pitch............................................. 257

Pitch, Burgundy ............................................ 257

Plasters...

Pomegranate.................................................. 257

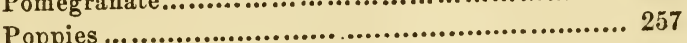

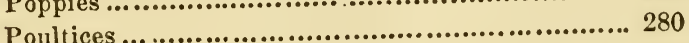

Potash

Potash, Caustic............................................ 257 
Potash, Carbonate of..................................... 258

Potash, Chlorate of....................................... 258

Potash, Iodide of........................................ 258

Potash, Nitrate of......................................... 258

Potash, Sulphate of................................... 258

Potash, Sulphuret of................................... 258

Prescriptions................................................ 274

Prussic Acid.......................................... 258

Pumpkin Seeds......................................... 258

Purgatives.................................................. 258

Pyroxylic Spirit..................................... 258

Q.

Quinine

$.227-258$

R.

Rennet......................................... 256, 258

Rhubarb............................................. 259

Ricinus Communis...............................223, 259

Rochelle Salts........................................... 259

Rosin .............................................. 270

S.

Saffron ....................................... 227, 259

Sugapenum ........................................... 259

Sal Ammoniac.................................... 215-259

Salt..................................................... 259

Sal Volatile............................................... 259

Sassafras ................................................. 259

Sarracenia............................................. 259

Savin..................................................... 259

Sedatives................................................. 260

Senega................................................... 260

Sialagogues............................................. 261

Silver................................................... 261

Silver, Nitrate of......................................... 261

Snake Root ............................................. 260

Soap ......................................................... 260

Soap Liniment.......................................... 261

Soda ...................................................... 261 
Soda, Carbonate of....................................... 261

Soda, Sulphate of....................................... 261

Soda, Sulphite of ..................................... 261

Spanish Fly ............................................... 262

Spanish Fly Ointment.................................... 263

Specifics.................................................... 263

Spermaceti.................................................. 263

Spirits of Nitre.......................................... 264

Spirits of Wine.......................................... 264

Spirits........................................................ 282

Sponge.................................................... 264

Starch .................................................... 264

Styptics .................................................. 265

Sudorifics .................................................. 265

Sugar of Lead............................................. 265

Sugar of Milk............................................... 265

Sulphuric Acid........................................... 212

Sulphur ................................................. 265

Sulphurous Acid Gas..................................... 266

Sulphate of Zinc......................................... 267

Sweet Spirit of Nitre................................... 264

T.

Tannin...................................................... 267

Tannin, Glycerine of.................................. 267

Tannin, Krameria of.................................... 267

Table of Doses............................................. 285

Tar .......................................................... 268

Tar, Oil of................................................. 268

Tartar Emetic............................................... 268

Tinctures.................................................... 281

Tobacco .................................................... 268

Tragacanth............................................ 238-268

Turpentine................................................... 268

Turpentine, Common.................................... 269

Turpentine, Canada........................................ 269

Turpentine, Venice ....................................... 269

Turpentine, Oil........................................... 269

U.

Urva Ursi................................................. 270 
V.

Valerian..................................................... 270

Valerian and Iron........................................ 271

Valerian and Quinine .................................. 271

Veratrum................................................. 271

Veratria.................................................... 271

W.

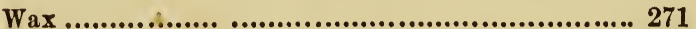

Waters ........................................................ 282

Willow Bark............................................. 272

$\mathbf{Y}$.

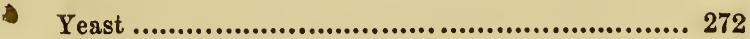

Z.

Zinc........................................................ 272

Zinc, Oxide of............................................. 273

Zinc, Carbonate of........................................ 273

Zinc, Chloride of.......................................... 273

Zinc, Sulphate of. ........................................ 273

Zinc, Acetate of.......................................... 274

Zingiberis ................................................. 274

Zylodine.................................................. 274 


\section{PART III.}

A.

Abortion................................................... 292

Abortion, Epizootic.................................... 295

Abortion, Sporadic..................................... 295

Abomasum............................................... 291

After-birth................................................ 311

Anæmia.................................................. 296

Angle Berries........................................... 296

Anthrax................................................. 327

Apoplexy.............................................. 297

Aphtha...................................................., 297

B.

Biliary Gall-stones ..................................... 297

Biliary Gall-stones, Yellow............................ 297

Biliary Gall-stones, Dark ............................ 297

Biliary Gall-stones, White ............................. 297

Black Quarter............................................ 297

Black Tongue ............................................. 298

Black Water................................................ 298

Bladder. Diseases of...................................... 298

Bladder, Prolapsus of.................................... 299

Blain......................................................... 327

Blood-shot........................................ 297, 300

Blood Diseases ..................................... 300, 328

Brain Diseases ............................................ 300

Brittany Cow............................................ 300

Bronchitis ................................................. 301

Bull Burnt................................................. 304 
C.

Cachexia.................................................... 305

Calving .................................................... 306

Calving, Natural ..................................... 307

Calving, Unnatural..................................... 308

Catarrh ................................................... 313

Catarrhus, Linuum Frontalium........................ 313

Cattle Plague............................................... 313

Chicken Pox.................................................... 314

Choking ................................................ 314

Cleansing ................................................. 311

Colic......................................................... 315

Consumption ............................................... 315

Contagious Typhus...................................... 317

Coryza.................................................. 313, 317

Cow Pox....................................................... 317

Croup......................................................... 317

Cud, Loss of.............................................. 318

Diarrhœa.................................................. 319

Diarrhœa in Calves......................................... 320

Diarrhœa, Chronic...................................... 319

Diarrhœa, Simple...................................... 319

Distention of the Rumen.............................. 321

Dropping after Calving.......................... 321, 336

Dysentery..................................... 315, 321

E.

Ectopia Cordis.......................................... 321

Epizootic Aphtha................................. 321, 338

Eye Diseases.......................................... 323

F.

Falling Sickness................................. 323, 336

Falling of the Womb..................................... 323

Falling of the Calf Bed................................... 323

Fardel Bound............................. 325, 330, 333

Fever..................................................... 325

Foul Claw............................................... 325

Foul in the Foot...................................... 325 
G.

Gangrene of the Tail......................................... 325

Garget.......................................................... 326

Gastro-Enteritis in Calves ......................... 320, 327

Glossitis.................................................... 327

H.

Hæmaturia......................................... 300, 328

Hair Balls .................................................... 328

Herpes ............................................ 329, 836

Hide Bound ................................................. 329

Hoose in Calves ............................................... 329

Hoven....................................................... 350

Hydatids in the Brain...................................... 331

I.

Inflammation................................................ 332

Impaction of the Omasum................................ 333

J.

Jaundice....................................................... 334

K.

Kidney Disease.............................................. 385

I.

Laryngitis....................................... 301, 317, 335

Leucorrhøa.......................................... 335, 361

Lice............................................................. 335

Liver Diseases....................................... 334, 335

Lock-Jaw..................................................... 335

Lung Diseases......................................... 335, 340

Lymphangitis................................................. 335 
M.

Malignant Catarrh..................................... 313

Mammitis............................................326, 336

Mange..................................................... 336

Milk Fever....................................... 323, 336

Milk Trembles.......................................... 338

Moor Evil............................. 225, 330, 333, 338

Mouth Diseases........................................... 338

Murrain.......................................... 321, 338

N.

Nephritis............................................... 339

Nervous Diseases ....................................... 339

o.

Estromania.............................................. 339

Estrus Bovis. ........................................... 339

Osseous................................................... 339

Ovarian Dropsy......................................... 339

Ovarian Tumor....................................... 339

P.

Palsy ..................................................... 340

Paralysis................................................... 340

Parasitic Disease................................. 329, 340

Parturient Fever............................ 323, 336, 340

Phthisis............................................. 315, 340

Placenta............................................ 311, 340

Plethora..................................................... 340

Pleurisy .................................................. 340

Pleuro-pneumonia ..................................... 340

Prolapsus Vaginæ.......................................... 346

Prolapsus Vesicæ...................................... 346

Prurigo Vernalis ........................................ 346

Puerpural Fever ............................. 323, 336, 346 
Q.

Quarter Ẹvil.

R.

Red Water $300,328,346$

Reticulum....

Retroflexion of the Womb.

Retroversion of the Womb................................ 347

Rheumatism................................................. 348

Rinderpest...................................................... 348

Ring Worm................................................. 348

Rot ....................................................... 348

Rumen.............................................297, 348

Rupture in Calves.......................................... 348

\section{s.}

Sheep Diseases.............................................. 365

Siberian Boil Plague.......................................... 349

Slinking the Calf......................................292, 349

Sore Throat..............................................301, 349

Sore Teats.................................................. 352

Spaying ....................................................... 349

Spaying Cows................................................. 349

Splenic Apoplexy............................................. 351

Spring Eruption.......................................348, 352

Stomach Staggers......................................... 352

Strangulation ........................................314, 352

Sturdy ..........................................331, 352

\section{T.}

Teats, Sore........................................... 552

Teeth Diseases............................................. 353

Texan Fever.............................................. 353

Throat Diseases.........................................301, 354

Thrush..................................................297, 354

Tongue......................................................... 354 
Tubercle Diseases....................................319, 354 Tympanitis ...........................................330, 354

Typhus Contagiosus Boum............................ 355

U.

Udder Diseases.......................................... 360

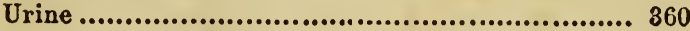

V.

Vaginal Catarrh................................................ 360

Varicella Boum........................................... 360

Vertigo...............................................352, 360

W.

Warbles....................................................... 360

Water in the Chest...................................340, 361

Whites.................................................335, 361

Worms................................................329, 361

Womb.....................................323, 347, 361 


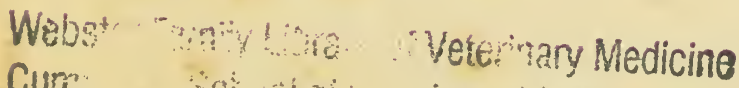

Cur win mary wedicine at

Tuits

$200 \mathrm{~V}$ 




5 
W.

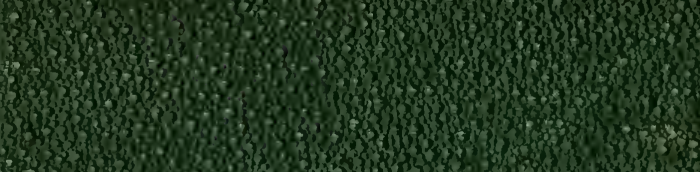

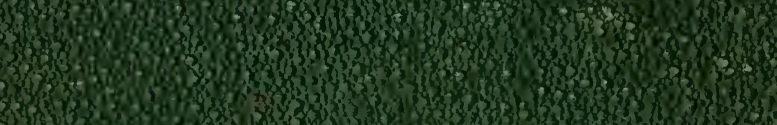

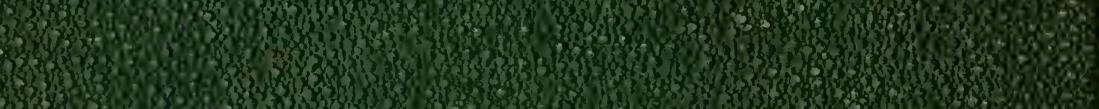

3353 .

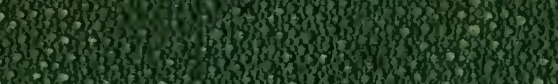

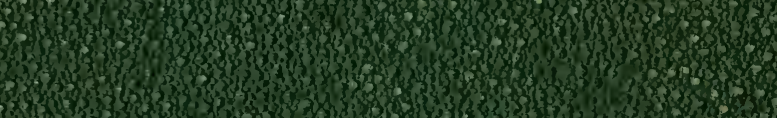

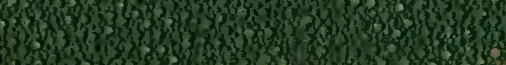

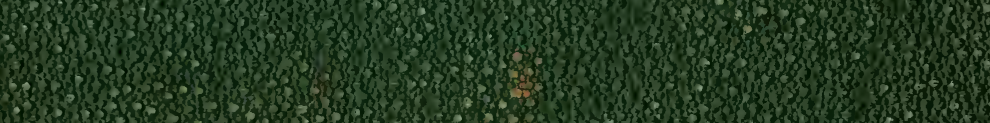

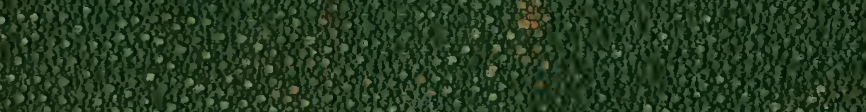

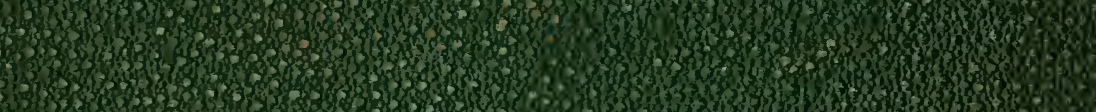

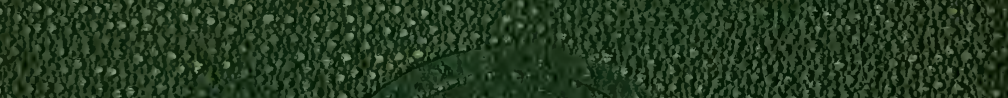

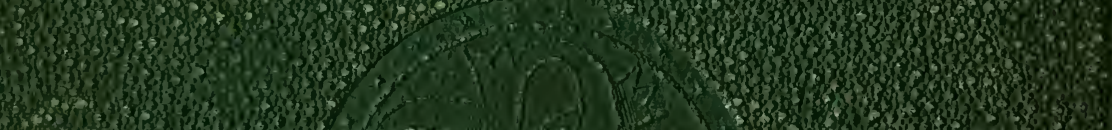

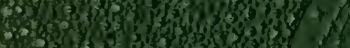

Q

(2)

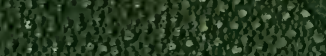

H. 35 .

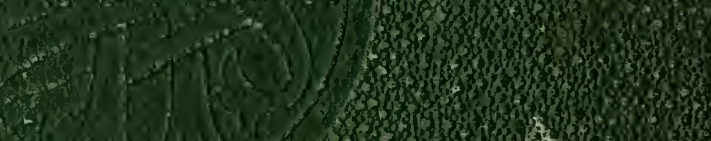
3.

2.

(1)

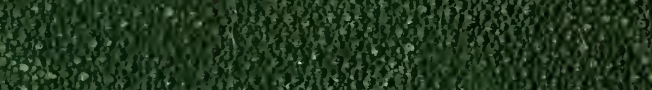

1.

20353 .

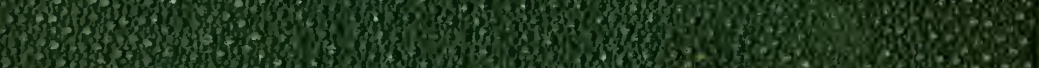

(x)

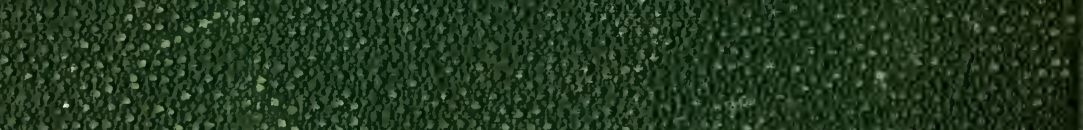

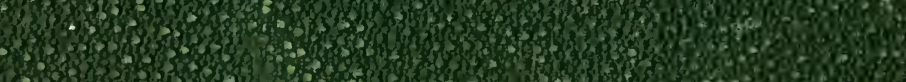

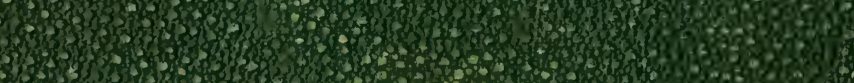

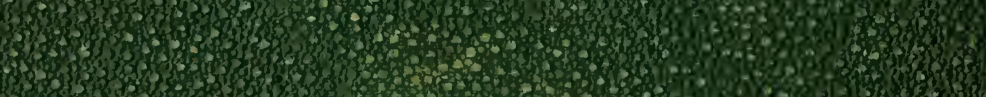

1075 .

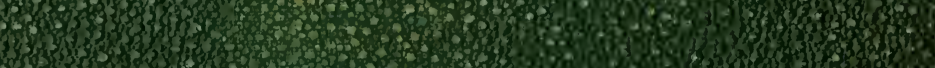

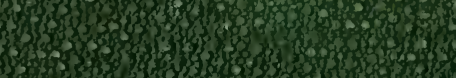

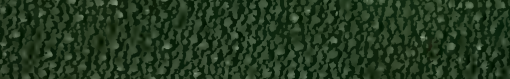

s. 35,3 .

3. 3 (n) 